\title{
Biology of Odoriferous Defensive Stink Glands of the Red Flour Beetle Tribolium castaneum
}

\author{
Dissertation \\ for the award of the degree \\ "Doctor rerum naturalium" (Dr.rer.nat.) \\ of the Georg-August-Universität Göttingen \\ within the doctoral program "Biology" \\ of the Georg-August-University School of Science (GAUSS) \\ submitted by \\ Sabrina Lehmann \\ from Hildesheim
}

Göttingen, 2015 


\section{$\underline{\text { Thesis Committee }}$}

Prof. Dr. Ernst A. Wimmer Department of Developmental Biology, Johann-FriedrichBlumenbach-Institute of Zoology and Anthropology, GeorgAugust-Universität Göttingen

Prof. Dr. Ivo Feußner

Department of Plant Biochemistry, Albrecht-von-Haller-Institute of Plant Sciences, Georg-August-Universität Göttingen

\section{Members of the Examination Board}

Reviewer:

Prof. Dr. Ernst A. Wimmer, Department of Developmental Biology, Johann-Friedrich-Blumenbach-Institute of Zoology and Anthropology, Georg-August-Universität Göttingen

Second Reviewer: $\quad$ Prof. Dr. Ivo Feußner, Department of Plant Biochemistry, Albrecht-von-Haller-Institute of Plant Sciences, Georg-AugustUniversität Göttingen

Further members of the Examination Board:

Prof. Dr. Ralf Heinrich

Department of Cellular Neurobiology, Johann-FriedrichBlumenbach-Institute of Zoology and Anthropology, GeorgAugust-Universität Göttingen

Prof. Dr. Andreas Stumpner Department of Cellular Neurobiology, Johann-FriedrichBlumenbach-Institute of Zoology and Anthropology, GeorgAugust-Universität Göttingen

Dr. Roland Dosch Department of Developmental Biochemistry, Universitätsmedizin Göttingen

Dr. Nikola Prpic-Schäper Department of Developmental Biology, Johann-FriedrichBlumenbach-Institute of Zoology and Anthropology, GeorgAugust-Universität Göttingen

Day of Oral Examination: $\quad$ August $21^{\text {st }}, 2015$ 


\section{Authorship Declaration}

I hereby declare that the dissertation "Biology of Odoriferous Defensive Stink Glands of the Red Flour Beetle Tribolium castaneum" was written by myself and is based on my experimental work in the department of Developmental Biology, Georg-August-University Göttingen, with no other sources and help than quoted. It has not been submitted elsewhere for the award of any other doctoral degree.

Göttingen,

Parts of this work have already been published:

- Jianwei Li, Sabrina Lehmann, Bernhard Weißbecker, Irene Ojeda Naharros, Stefan Schütz, Gerrit Joop, and Ernst A. Wimmer (2013) Odoriferous Defensive Stink Gland Transcriptome to Identify Novel Genes Necessary for Quinone Synthesis in the Red Flour Beetle, Tribolium castaneum. PloS Genetics 9 (7)

- Christian Schmitt-Engel, Dorothea Schultheis, Jonas Schwirz, Nadi Ströhlein, Nicole Troelenberg, Upalparna Majumdar, Van Anh Dao, Daniela Grossmann, Tobias Richter, Maike Tech, Jürgen Dönitz, Lizzy Gerischer, Mirko Theis, Inga Schild, Jochen Trauner, Nikolaus D.B. Koniszewski, Elke Küster, Sebastian Kittelmann, Yonggang Hu, Sabrina Lehmann, Janna Siemanowski, Julia Ulrich, Kristen A. Panfilio, Reinhard Schröder, Burkhard Morgenstern, Mario Stanke, Frank Buchholz, Manfred Frasch, Siegfried Roth, Ernst A. Wimmer, Michael Schoppmeier, Martin Klingler \& Gregor Bucher (2015) The iBeetle large-scale RNAi screen reveals gene functions for insect development and physiology. Nat Commun.6 

"Sometimes the wrong choices bring us to the right places"

(Unknown) 



\section{Danksagung}

Als erstes sei hier meine Familie erwähnt. Vielen Dank für euer Vertrauen, Verständnis und eure Hilfe zu jeder Zeit und in allen Lebenslagen. Ohne eure Unterstützung wäre dieser Weg sehr viel beschwerlicher gewesen!

Als nächstes möchte ich meinem Betreuer Professor Dr. Ernst A. Wimmer danken, der mir die Chance gab als Nicht-Entwicklungsbiologin in der Abteilung für Entwicklungsbiologie zu promovieren. Danke für die Diskussionsbereitschaft zahlreicher Ergebnisse und neuer Experimente und für den Raum zur Entwicklung vieler eigener Ideen, die letztlich richtungweisend für diese Arbeit geworden sind.

Ich danke außerdem meinem zweiten Betreuer und Korreferenten Professor Dr. Ivo Feußner für seine beratende wie motivierende Funktion und seine zahlreichen Hilfestellungen bei Problemen und Fragen biochemischer Natur.

Ein großer Dank geht auch an Professor Dr. Gregor Bucher für seine Gesprächsbereitschaft in allen Lebens- und iBeetle-Lagen, sowie Dr. Nico Posnien, Dr. Nikola-Michael Prpic-Schäper und Professor Dr. Sigrid Hoyer-Fender für ihre konstruktive Kritik an meinem Forschungsprojekt.

Einen nicht zu unterschätzenden Anteil an dieser Arbeit haben die (Vor)Leistungen von Dr. Jianwei Li, Elke Küster, Margret Winkler und des iBeetle-Konsortiums. Vielen Dank für eure tolle Arbeit und Unterstützung!

Ein ebenso großer Dank geht an meine Studenten Zhiyuan Shi, Katja Rust und Tobias Vollmer für ihre Leistungsbereitschaft und Bereicherung meines Projekts sowie an Bibi Atika für die Fortführung der Erforschung der Stinkdrüsen.

Der gesamten Abteilung Entwicklungsbiologie danke ich für eine besondere kollegiale Arbeitsatmosphäre und Hilfsbereitschaft untereinander. Ganz besonders möchte ich Janna, Alice, Bernhard und Ingrid hervorheben, die für mich mehr als nur Kollegen geworden sind. Danke für 3,5 Jahre voller verrückter Unterhaltungen, kreativer Zombievernichtungsschlachten, feucht-fröhlicher Doppelkopfabende, lautstarker Eurovision-Songcontest-Ekstasen und vieles mehr!

Nicht zuletzt danke ich meinen Freunden Sabine, Inga und Stefan für unsere legendären Mittwochabende voller Spiel, Spaß und gutem Essen (und Schokolade!). Außerdem möchte ich Sabine für die gemeinsame Erkundung fremder Kulturen, ihre zahlreichen (unentgeltlichen) Chauffeursdienste und die kritische Korrektur dieser Arbeit danken. Vielen Dank euch allen für eine tolle gemeinsame Zeit! 



\section{Table of contents}

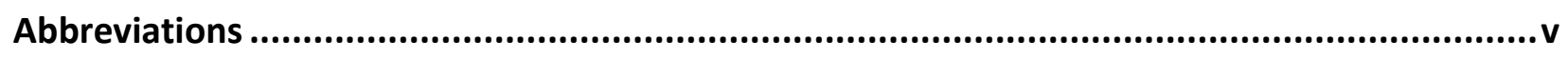

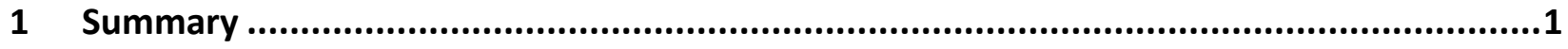

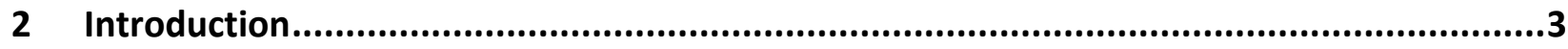

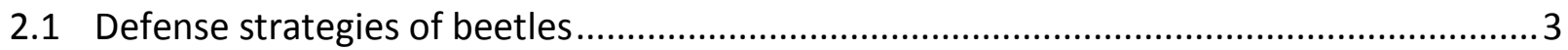

2.2 Chemical defense of $T$. castaneum .......................................................................

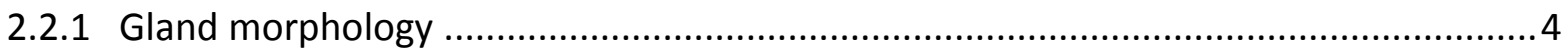

2.2.2 Gland secretion composition ..................................................................... 6

2.3 The red flour beetle as model insect for molecular studies on stink glands ....................7

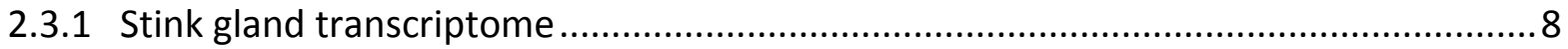

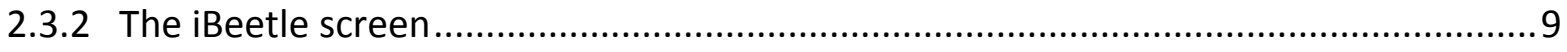

2.3.3 A Gal4-based enhancer trap screen .............................................................

2.4 Benzoquinones in beetle defensive secretions.......................................................... 10

2.4.1 Characteristics and molecular functions................................................... 10

2.4.2 The need for an adequate barrier for self-protection ........................................ 11

2.4.3 Model for benzoquinone production in T. castaneum ......................................... 11

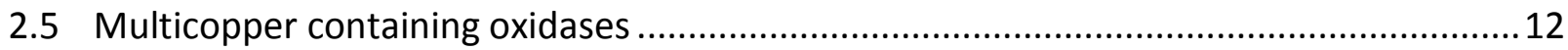

2.5.1 Laccase 2 - a phenol-oxidizing enzyme in the insect cuticle ..................................13

2.5.2 Tyrosinase - the phenoloxidase required for insect innate immune response .........14

2.5.3 Overlapping substrate specificities of phenol-oxidizing enzymes in the beetle .........16

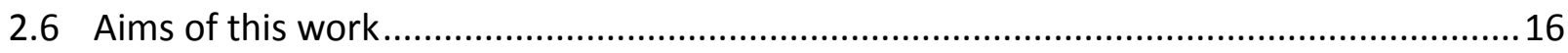

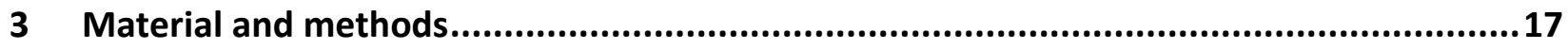

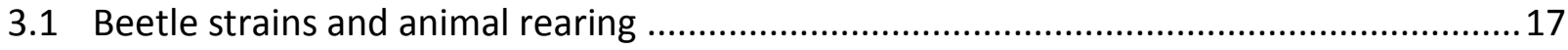

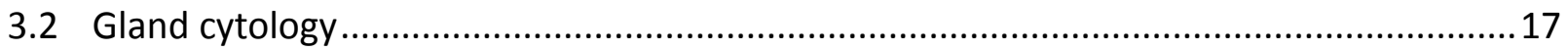

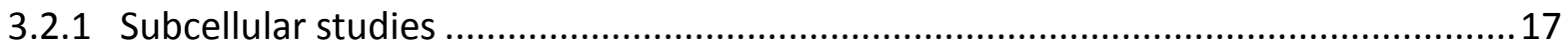

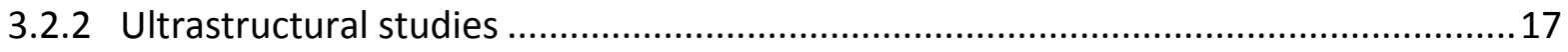




\section{TABLE OF CONTENTS}

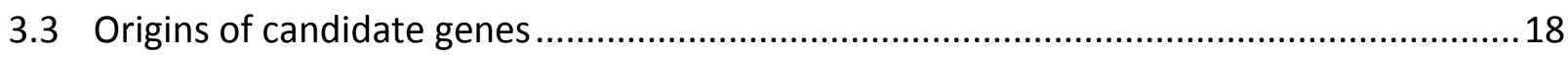

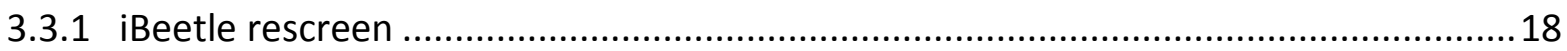

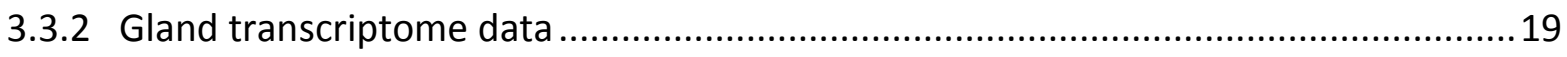

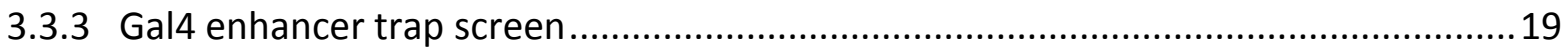

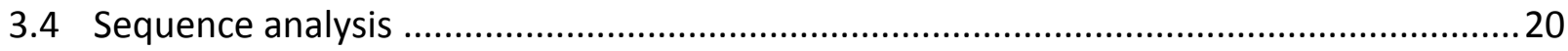

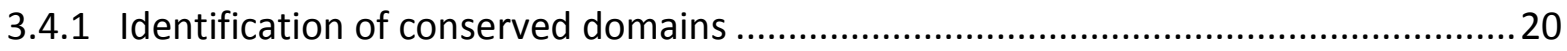

3.4.2 Prediction of subcellular location of proteins..................................................20

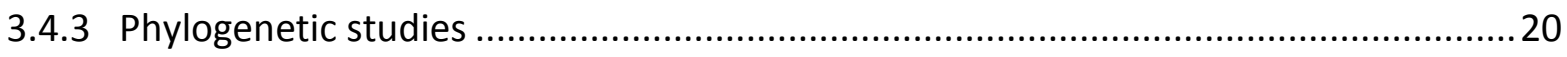

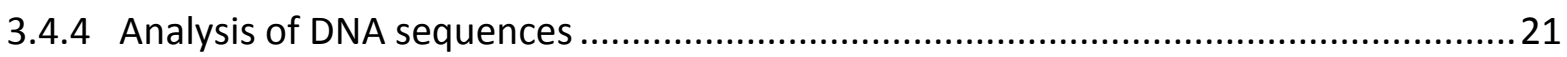

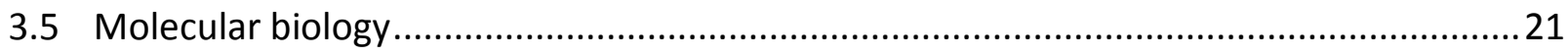

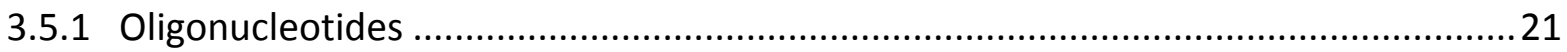

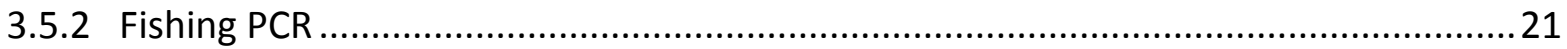

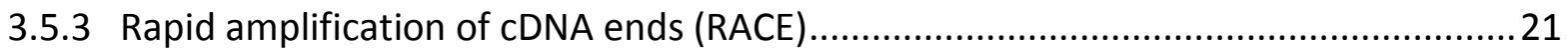

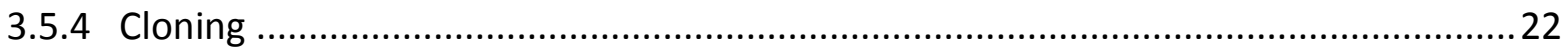

3.6 RNA interference-mediated gene knockdown....................................................22

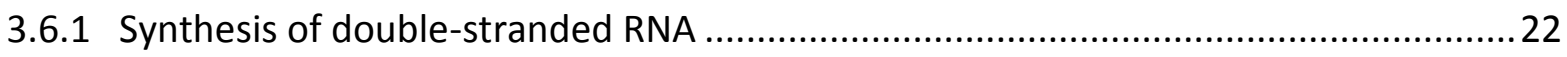

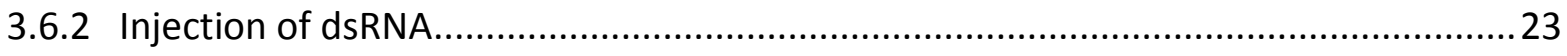

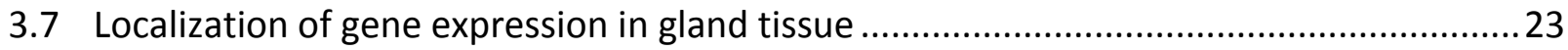

3.7.1 Synthesis of digoxigenin (DIG) -labeled RNA probes ........................................ 23

3.7.2 Gland whole mount in situ hybridization.......................................................... 23

3.8 Gland whole mount immunohistochemistry ..................................................... 25

3.9 Semi-quantitative gas chromatography-mass spectrometry analysis of gland volatiles ...26

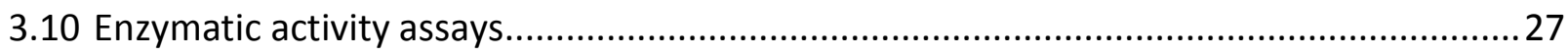

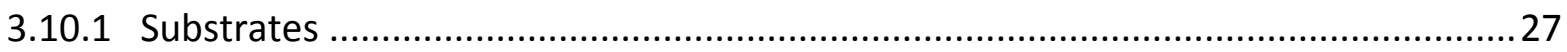

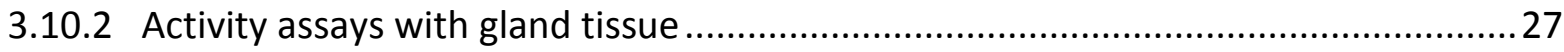

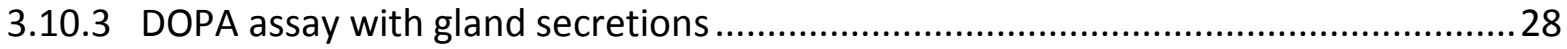

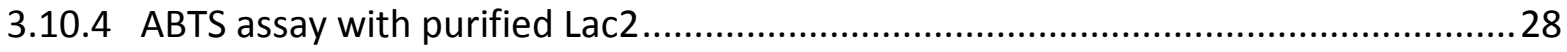




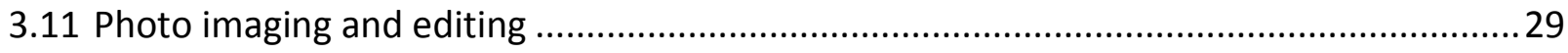

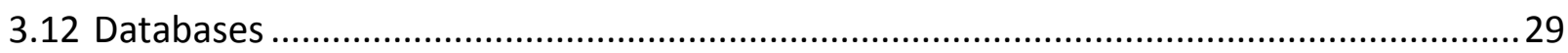

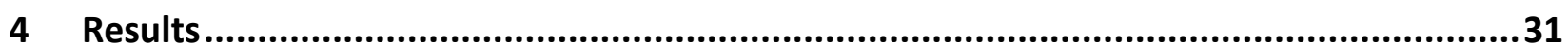

4.1 Gland cytology

4.1.1 Structure of secretion producing gland cells ..............................................................

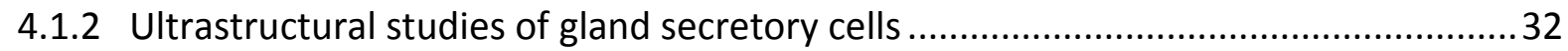

4.2 Screens for the identification of genes involved in stink gland biology ............................35

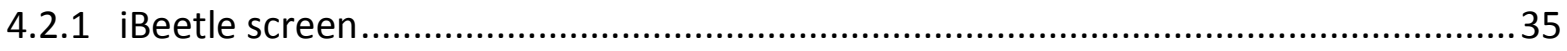

4.2.2 Candidate genes from a Gal4-based enhancer trap screen ...................................42

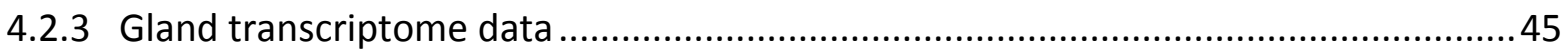

4.3 Gas chromatography-mass spectrometry analysis of gland secretion volatiles................53

4.4 iBeetle and enhancer trap screen versus transcriptomics ..............................................59

4.5 Localization of candidate gene expression in gland cells.................................................60

4.5.1 Expression analysis via in situ hybridization ............................................................6

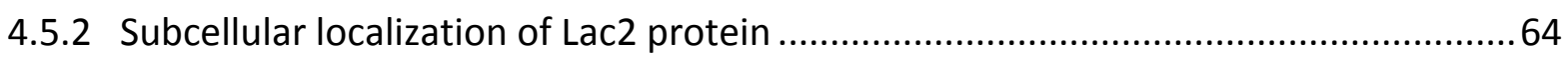

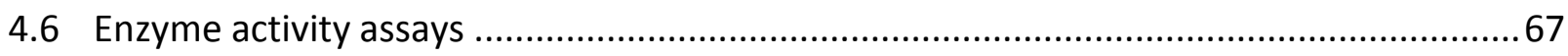

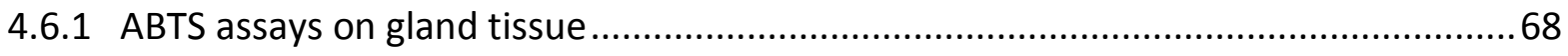

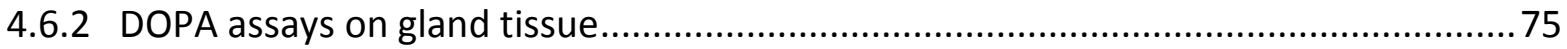

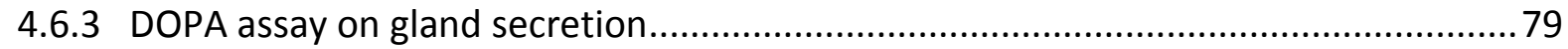

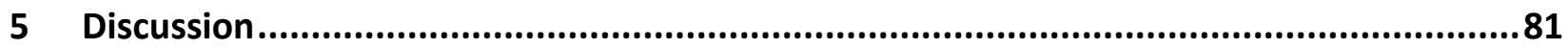

5.1 Stink gland morphology of $T$. castaneum and E. longicollis is highly similar .....................81

5.2 Prothoracic glandular $2 \mathrm{~b}$ cells display gene expression pattern from cell-type 1 in

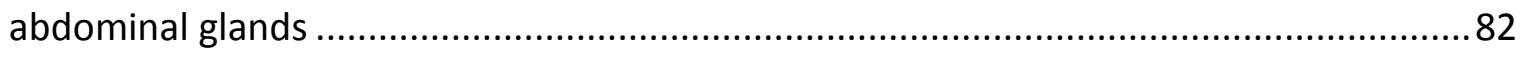

5.3 iBeetle as platform for the identification of genes related to gland secretion production

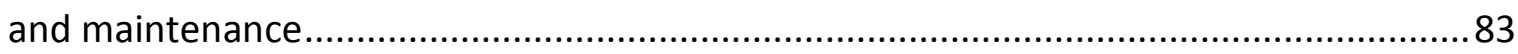

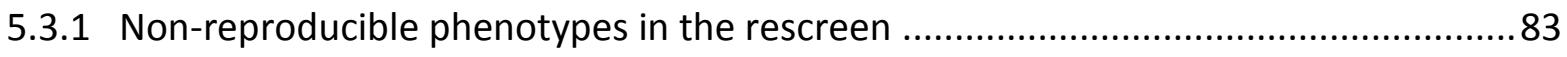

5.3.2 Rescreen results provided the basis for a more slender second screening phase.......84 


\section{TABLE OF CONTENTS}

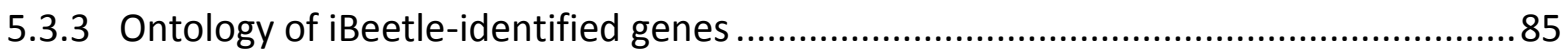

5.3.4 iBeetle-identified novel genes involved in stink gland secretion production .............85

5.4 Genes influencing the chemical composition of stink gland secretion .........................87

5.4.1 Four uncharacterized BQ-less genes and their possible molecular function .............87

5.5 Phenoloxidases in T. castaneum stink gland tissue .................................................91

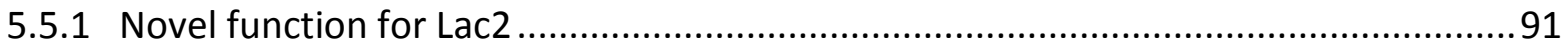

5.5.2 Tyr1 plays a (minor) role in stink gland benzoquinone production...........................95

5.6 Model about benzoquinone production in Tribolium stink glands..............................96

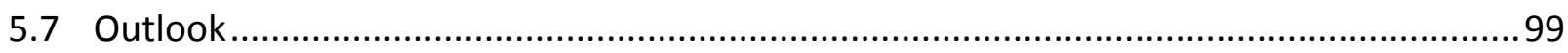

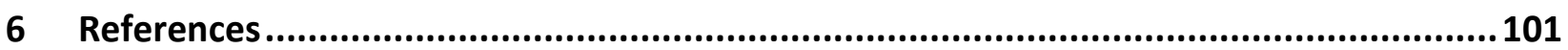

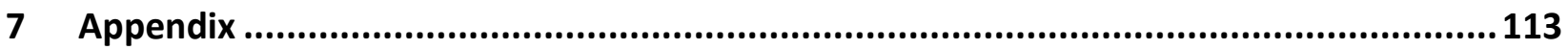

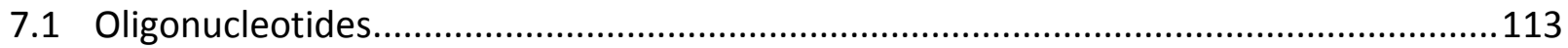

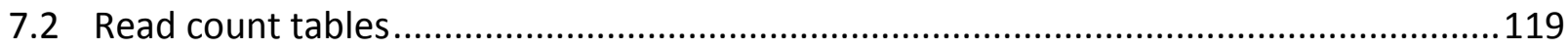

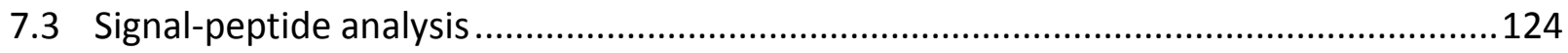

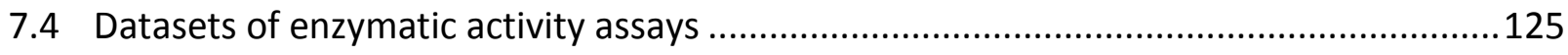

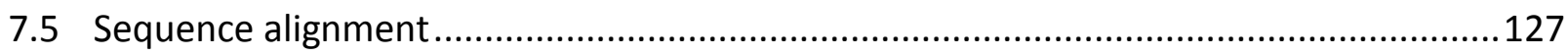

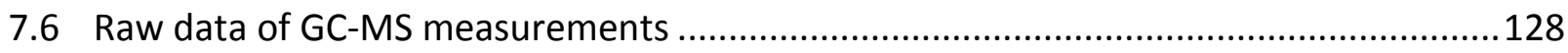

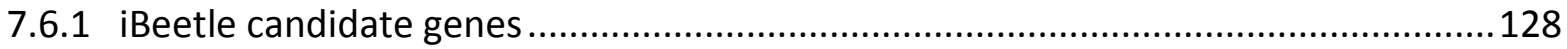

7.6.2 Candidate genes from transcriptome data and enhancer trap screen ...................130

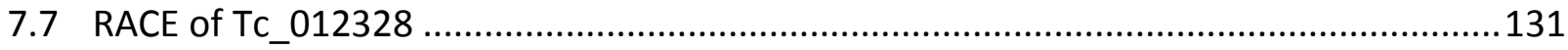

\section{Curriculum vitae}




\section{Abbreviations}

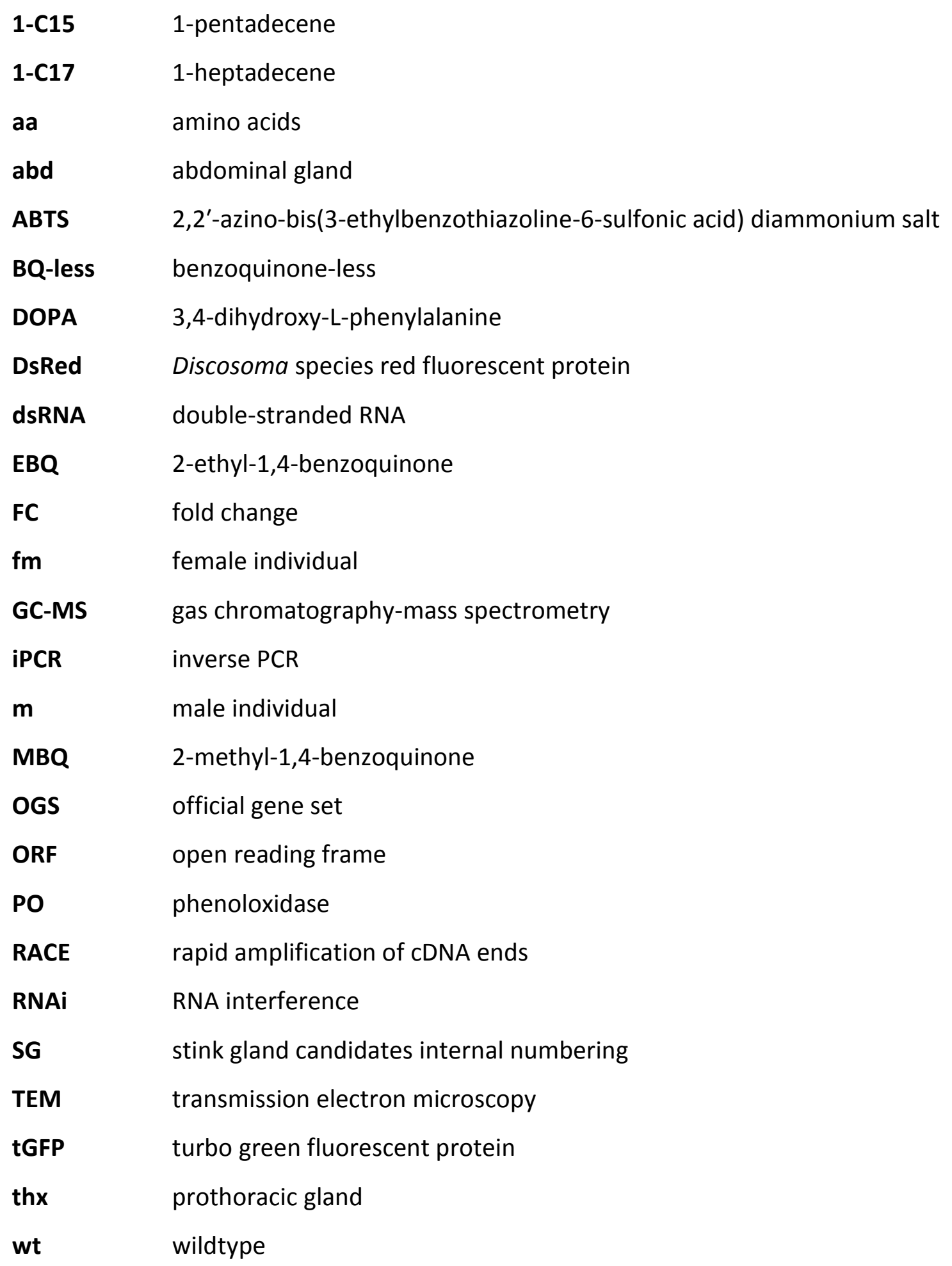





\section{Summary}

Chemical warfare is a very common defense strategy in the insect world. A broad range of coleopteran beetles react to predators, invaders, and parasitic microbes with the release of toxic and repellent substances, which are synthesized in specialized secretory organs, referred to as odoriferous defensive stink glands. The worldwide known pest beetle Tribolium castaneum (Coleoptera: Tenebrionidae) uses these glands to produce antimicrobial $p$-benzoquinones and 1alkenes. The stink gland morphology has been studied in detail in other tenebrionid beetles in the past, but to date only little is known about the genes involved in the biochemical processes of gland secretion production.

Here, I present first transmission electron microscopic pictures of $T$. castaneum stink gland cells and a subset of genes required for proper benzoquinone production. I selected 59 potential candidates from a genome-wide RNAi knockdown screen named "iBeetle" and 12 genes based on the analysis of 11 stink gland-related Gal4 enhancer trap lines. All 71 candidate genes were functionally characterized by RNAi-mediated gene knockdown. 34 of them were found to cause stink gland phenotypes upon knockdown and were therefore selected for a subsequent gas chromatography-mass spectrometry (GC-MS) analysis of secretion volatiles in respective RNAi knockdown glands. In total, I identified 14 candidate genes that displayed strongly altered glands in terms of secretion color and chemical composition upon knockdown, revealing their involvement in secretion biosynthesis. Three of them play an essential role specifically in benzoquinone biosynthesis. Based on stink gland transcriptome data, I additionally analyzed the function of four glucosidases, eleven peroxidases, as well as four phenoloxidases via RNAimediated gene knockdowns. Morphologically altered stink glands were found for one peroxidase (Peroxiredoxin 3, Prxd3) and two phenoloxidases (Tyrosinase 1, Tyr1; Laccase 2, Lac2). Subsequent GC-MS analysis of secretion volatiles revealed reduced benzoquinone levels in Prdx3 and Tyr1 knockdowns and a complete lack of benzoquinones in the knockdown of Lac2. For Lac2, I could detect its expression in a certain subgroup of secretory stink gland cells and confirm its involvement in $p$-benzoquinone biosynthesis by enzymatic activity assays on stink gland tissue. This indicates a so far unidentified role of the cuticle tanning phenoloxidase Lac2. 

INTRODUCTION

\section{Introduction}

\subsection{Defense strategies of beetles}

With approximately 400,000 described species, beetles (Coleoptera) are the species-richest order in the class of insects (Chapman, 2009). They colonize almost every habitat on earth and evolved different strategies to cope with pathogens, parasites, and predators. To decrease the danger of desiccation and the infestation by microorganisms, most beetles possess a strongly sclerotized exoskeleton (Eisner et al., 1961; Schierling and Dettner, 2013). However, to prevent being caught by vertebrate predators, more sophisticated tactics are needed in addition. Some beetles are true camouflage experts, whereas others are painted in eye-catching warning colors to demonstrate their inedibility already from a distance. According to this, mimicry is also a practiced defense strategy among beetles (Dettner and Peters, 2003). Interestingly, some beetles escape from dangerous situations by playing dead (thanatosis), like the ladybird beetle, who in addition releases a foul-smelling drop of his hemolymph from the knee joints (reflex bleeding) to simulate a decayed state (Grill and Moore, 1998). This type of chemical defense has been worked up in some beetle families through the development of specialized secretory organs referred to as odoriferous or stink glands (Tschinkel, 1975a), in which toxic and repellent chemicals are produced and released in case of emergency. The masters of chemical warfare are the bombardier beetles (Carabidae, e.g. species of the tribe Brachinini). They eject boiling noxious substances explosively from the tip of their abdomen precisely at approaching enemies. The reason for this enormous explosive force lies in the two-chamber-reaction mechanism, which allows the formation of chemical toxicants in a strongly exothermic reaction under a simultaneous massive liberation of oxygen gas (Aneshansley et al., 1969; Arndt et al., 2015; Beheshti and Mcintosh, 2007). The same toxic substances, $p$-benzoquinones and derivatives thereof, are also produced in the pest beetle T. castaneum (Loconti and Roth, 1953). 


\section{INTRODUCTION}

\subsection{Chemical defense of $T$. castaneum}

\subsubsection{Gland morphology}

Like several other Tribolium species (e.g. T. audax, T. brevicornis, T. confusum, T. destructor; Markarian et al., 1978), T. castaneum possesses two pairs of defensive stink glands, one in the prothorax and one in the most posterior part of the abdomen (Markarian et al., 1978; Roth, 1943). The prothoracic glands are located on the anterolateral region of the thoracic cavity, whereas the abdominal glands are attached to the last sternite of the abdomen (Sokoloff 1972, Figure 1). The stink glands of T. castaneum, T. confusum, and another tenebrionid beetle, Eleodes longicollis (only abdominal glands), consist of a secretion producing tissue and a reservoir for secretion storage (Eisner et al., 1964; Happ, 1968; Roth, 1943).

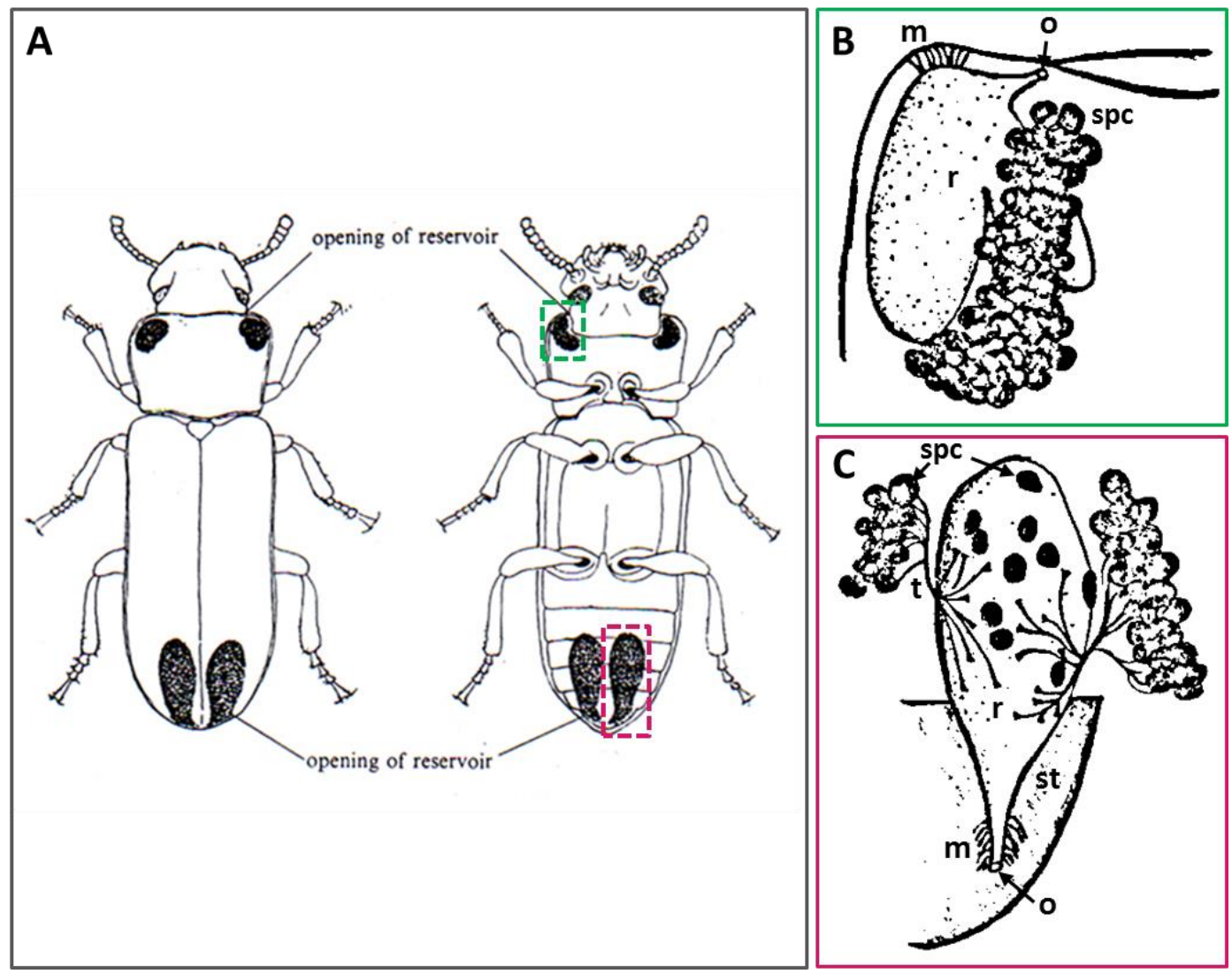

Figure 1: Morphology of stink glands in Tribolium. A Position of gland pairs and opening of the reservoir in T. confusum (picture taken from Sokoloff, 1972). B+C Schemes displaying structure and position of prothoracic (green frame) and abdominal gland (red frame) in T. castaneum (drawings are based on own observations). Note that the tubules, which exist in both gland-types and connect the secretion producing cells with the reservoir, are not indicated for prothoracic gland for clearness reasons. $\mathrm{m}$ : muscle, o: opening of reservoir, r: reservoir, spc: secretion producing cells, st: last abdominal sternite t: tubules. 
In abdominal glands, two different types of secretion producing cells are present (Figure 1C, Figure 2; Eisner et al., 1964; Happ, 1968; Roth, 1943; Sokoloff, 1972): Type 1 cells (cell 1) are individual or paired cells that are present over the whole surface of the reservoir except the apex. Each cell harbors a huge vesicle, which itself carries a simple cuticle-lined organelle. This cuticular or vesicular organelle constricts into a cuticle-lined efferent tubule that connects the lumen of the vesicular organelle with the reservoir. Whether or not this tubule is carried by another cell attached to cell 1 has been controversially discussed. For $T$. confusum, such a tubule-carrying cell has not been reported in the studies of Roth (1943). Eisner et al. (1964) only speculated about its existence in the glands of E. longicollis, whereas Happ (1968) described a reference diagram of the secretory cells in the defensive glands of $E$. longicollis and $T$. castaneum, which clearly depicts a tubule carrying cell (Figure 2). Also, the structure of the cuticular organelle is described differently. Eisner et al. (1964) and Happ (1968) suggested a division of the organelle in head and body for E. longicollis and T. castaneum, whereas Roth (1943) didn't find evidence for this in T. confusum.

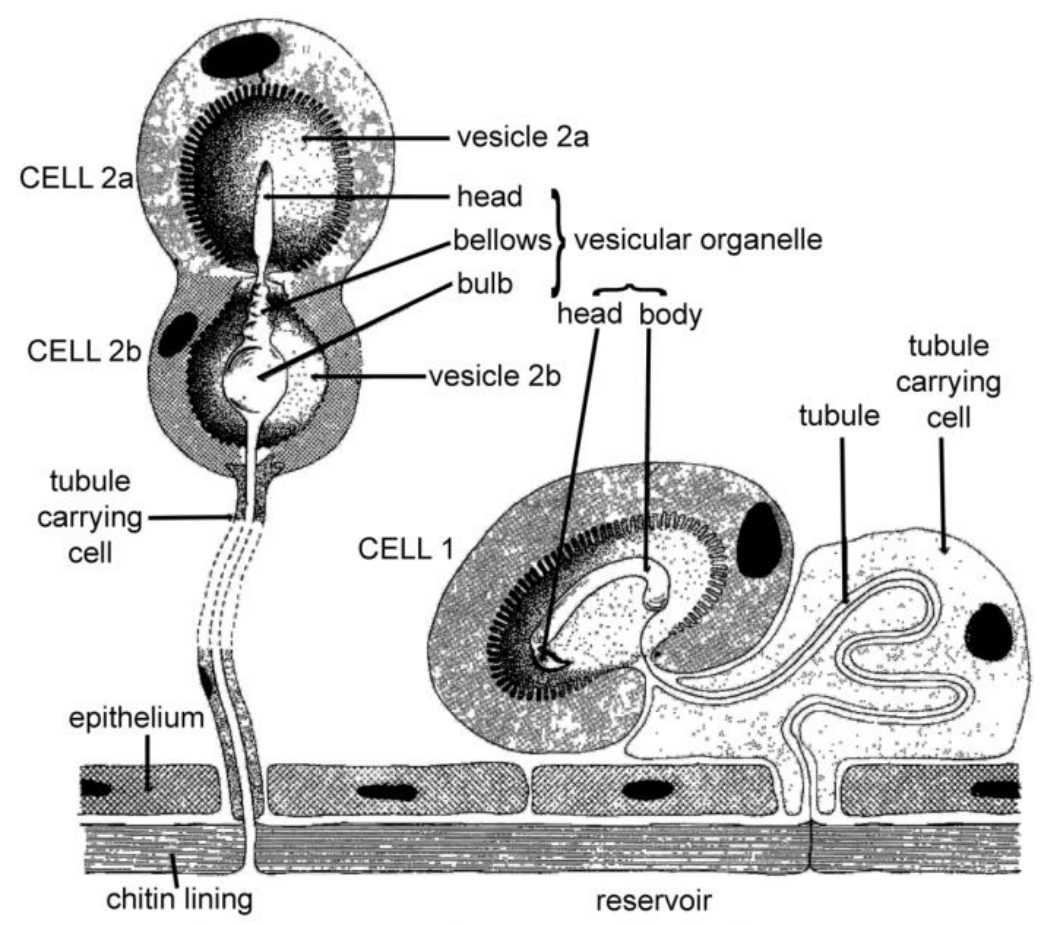

Figure 2: Reference diagram of secretion producing cells in defensive stink glands of $E$. longicollis and T. castaneum (Happ, 1968). 


\section{INTRODUCTION}

The second type of secretion producing cells (cell 2) aggregates into lobules. Each cell 2 consists of two fused cells $2 \mathrm{a}$ and $2 \mathrm{~b}$. Both have a nucleus and, similar to cell 1 , each carries a huge vesicle, $2 \mathrm{a}$ and $2 \mathrm{~b}$, respectively. Compared to cell-type 1 , the structure of the cuticular organelle is more complex here. It can be divided into a head lying inside the vesicle $2 a$, which constricts and then expands into the bulb, which is a rounded and strongly wrinkled structure and almost fills the vesicle $2 \mathrm{~b}$. The bulb constricts into a tubule, which runs down the cell aggregates and then enters the reservoir. These long canals do not fuse on their way and are the only structures that connect the lobules with the reservoir. In thoracic glands, cell-type 1 is absent (Figure 1B), whereas cell-type 2 is believed to have the same structure as cells 2 in abdominal glands (Roth, 1943). However, a detailed comparison of abdominal and prothoracic gland cell-type 2 has not been reported yet.

\subsubsection{Gland secretion composition}

Based on chemical tests and UV spectral analysis, Alexander and Barton (1943) as well as Loconti and Roth (1953) were the first to identify 2-methyl- and 2-ethyl-1,4-benzoquinones as the main components secreted by both sexes in prothoracic and abdominal glands of $T$. castaneum. Later, the same two substances have been detected via gas chromatography analysis in secretions of 147 further tenebrionid species (Tschinkel, 1975a). With the use of gas chromatography-mass spectrometry (GC-MS) analysis, Howard (1987) additionally identified the corresponding hydroquinones in secretions of eight Tribolium species including T. castaneum. Hydrocarbons represent the second group of secretion chemicals in several species of the genus Tribolium. 1-pentadecene was identified as the most prevalent non-quinone in secretion of $T$. castaneum, T. confusum, T. audax, T. brevicornis, T. destructor, T. madens, and T. freemani (Endt and Wheeler, 1971; Howard, 1987; Keville and Kannowski, 1975; Markarian et al., 1978; Tschinkel, 1975a). Additionally, Markarian et al. (1978) reported small amounts of 1,6pentadecadiene and two unidentified hydrocarbons for T. castaneum. Recent GC-MS studies revealed that abdominal and prothoracic glands of $T$. castaneum display the same spectrum of volatiles (Li et al., 2013), the main five being the para-benzoquinones 2-methyl-1,4benzoquinone (MBQ) and 2-ethyl-1,4-benzoquinone (EBQ), as well as the hydrocarbons 1- 
pentadecene, 1,8-heptadecadiene, and 1-heptadecene (Figure 3, red numbers). The level of EBQ is generally higher than MBQ (Li et al., 2013; Markarian et al., 1978; Pappas and Wardrop, 1996; Unruh et al., 1998), and secretion levels are similar in both T. castaneum sexes (Li, 2013).

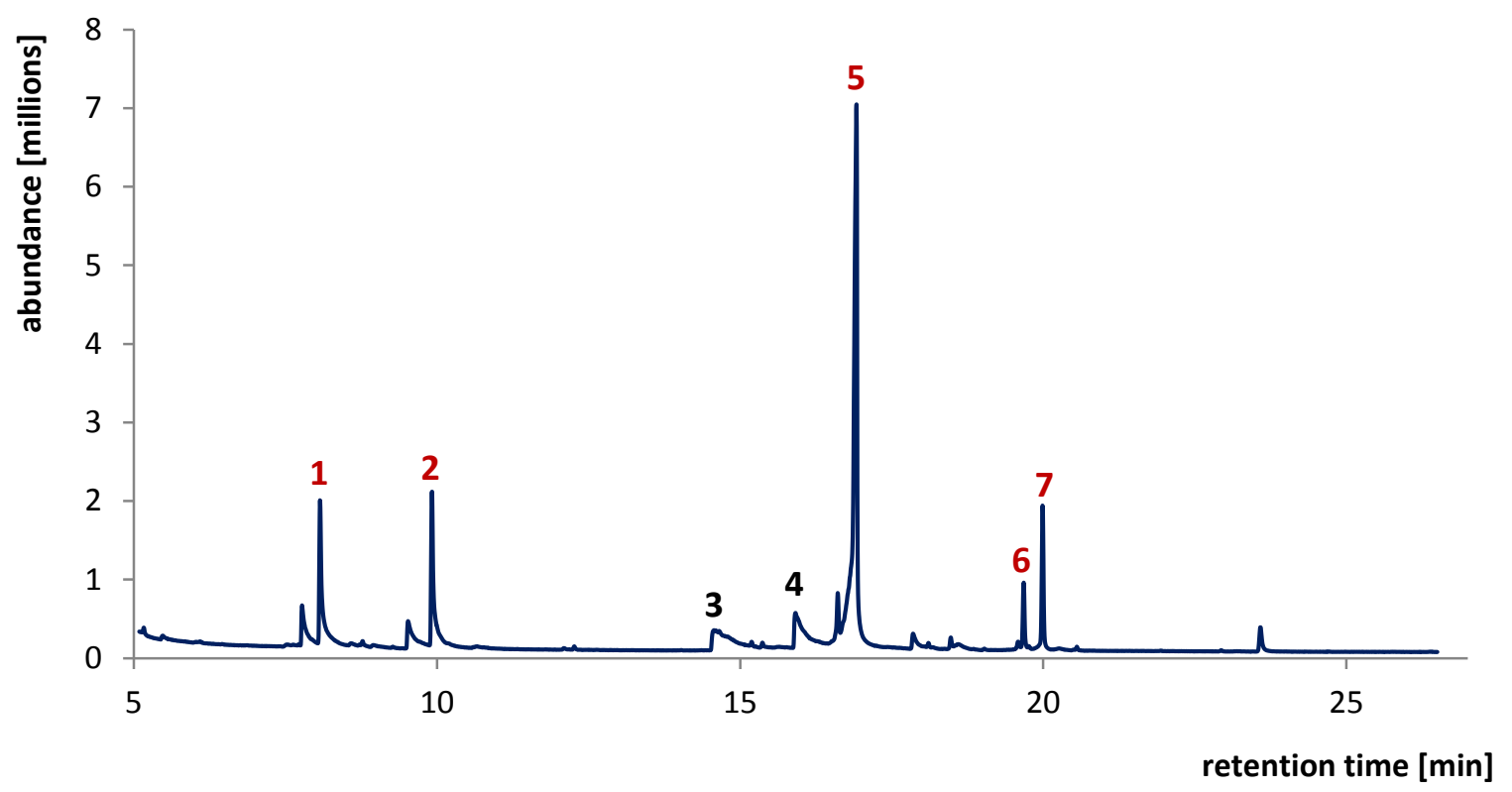

Figure 3: GC-MS chromatogram of wildtype abdominal stink glands. The peaks are: 1: 2-methyl-1,4benzoquinone (MBQ), 2: 2-ethyl-1,4-benzoquinone ( $E B Q)$, 3: 2-methyl-1,4-hydroquinone, 4: 2-ethyl-1,4hydroquinone, 5: 1-pentadecene, 6: 1,8-heptadecadiene, 7: 1-heptadecene. Traces of 1,6pentadecadiene, 1,2-dimethoxy-4-n-propylbenzene, and 1-hexadecene have been identified by Li et al. (2013), but are not indicated here. Red numbers highlight the five main volatiles. Note that double bond positions in 1,8-heptadecadiene have not been confirmed. They were predicted based on similar peaks obtained in previous studies (Görgen et al., 1990; Suzuki et al., 1975).

\subsection{The red flour beetle as model insect for molecular studies on stink glands}

The reasons for choosing $T$. castaneum to study stink glands on a molecular level are numerous and distinct. First, the red flour beetle is a ubiquitous pest of stored grain, flour, and other cereal products and prolific in developing resistances against insecticides (Brown et al., 2009). Thus, understanding the molecular functioning of its defense mechanism potentially providing a new basis for pest control is of economic-ecological importance. Secondly, its genome has been fully annotated (Richards et al., 2008) and several genetic tools are available, e.g. highly efficient methods for transposon-based genetic transformation (Berghammer et al., 1999; Lorenzen et 


\section{INTRODUCTION}

al., 2003, 2007), a GAL4/UAS expression system (Schinko et al., 2010), a heat-shock based misexpression system (Schinko et al., 2012) and, in contrast to the classical model insect Drosophila melanogaster, which does not possess stink glands, reversed genetics based on systemic RNAi response (Bucher et al., 2002; reviewed by Noh et al., 2012). Thus, in the last decade, $T$. castaneum has been evolved into a most sophisticated genetic model insect besides D. melanogaster.

Recently, the stink gland transcriptome of the red flour beetle has been published ( $\mathrm{Li}$ et al., 2013) and a genome-wide RNAi knockdown screen called "iBeetle" (Schmitt-Engel et al., 2015) as well as a Gal4 enhancer trap screen based on insertional mutagenesis (Trauner et al., 2009) are current projects to identify gene functions for insect development and physiology as well as to develop cell-type specific markers and drivers for targeted expression of transgenes, respectively. All candidate genes for this study were obtained from these three genome-wide approaches, which are shortly introduced in the following three subsections.

\subsubsection{Stink gland transcriptome}

First transcriptome data on beetle stink glands were provided by Li et al. (2013). Samples for mRNA sequencing on a next generation sequencing platform were wildtype male prothoracic glands, female prothoracic glands, male abdominal glands, and female abdominal glands. In addition, prothoracic glands of tar mutant displaying melanotic gland secretions were selected (Beeman et al., 1996). As non-gland reference served anterior abdomen tissue including e.g. muscle, gut, fat body, and cuticle. For each sample, about 28 million reads were obtained and half of them have been mapped to $T$. castaneum mRNAs of the official gene set in the BeetleBase (Kim et al., 2010; Wang et al., 2007). Comparative analysis of reads in stink gland and reference samples revealed 511 genes with differential expression in terms of gender, gland type and beetle strain (wildtype or mutant). From these, the authors functionally analyzed 77 genes that were at least $64 \mathrm{x}$ higher expressed in the glands compared to the reference tissue and identified three genes that are involved in benzoquinone synthesis in the beetle. Total reads for 16,645 official gene set numbers in every stink gland sample and the reference sample as well as corresponding calculated fold changes as index for gland specific differential expression, 
INTRODUCTION

were kindly provided by Dr. Jianwei Li for this study (Li et al., 2013; SRP040606 in the public database Sequence Read Archive (SRA)).

\subsubsection{The iBeetle screen}

The iBeetle screen is an RNAi-based, genome-wide, large-scale, and unbiased approach to identify novel genes involved in insect developmental and physiological processes (SchmittEngel et al., 2015). The first screening phase, which has already been completed, included a pupal injection screen and a larval injection screen. In the pupal screen, female pupae were injected and analyzed for late metamorphosis phenotypes. In addition, their offspring was examined for embryological defects. In contrast, in the larval screen, dsRNA-injected female larvae were observed for defects in early metamorphosis and general morphological abnormalities that occurred during adulthood, in particular alterations in ovaries and stink glands. After the first screening phase, 4480 genes had been analyzed in the larval screen and 5300 genes in the pupal screen, with reliable results for 3400 genes in both injection screens (Schmitt-Engel et al., 2015).

\subsubsection{A Gal4-based enhancer trap screen}

A Gal4-based enhancer trap screen for the identification of cell-type specific markers and drivers for targeted (over)expression of (trans)genes in T. castaneum is currently performed by Elke Küster in the laboratory of Professor Dr. Gregor Bucher (Georg-August-University Göttingen). Similar to Trauner et al. (2009), a Gal4-piggyBac-transposon is allowed to jump by crossing a mutator strain with a helper line providing active transposase. Subsequent integration of a UAS-turboGFP reporter construct visualizes the establishment of a new enhancer trap. Details are given in Figure 4. 


\section{INTRODUCTION}

$$
\begin{aligned}
& \frac{A^{\text {mut1 }}}{A^{\text {mut1 }}} \frac{B}{B} \frac{X}{Y} \times \frac{A}{A} \frac{B}{B} \frac{X^{M 26}}{X^{M 26}} \\
& \frac{A}{A^{\text {mut } 1}} \frac{B}{B} \frac{X^{M 26}}{Y} \times \frac{A}{A} \frac{B^{\text {UAS-tGFP }}}{B^{\text {UAS-tGFP }} \frac{X}{X}}
\end{aligned}
$$

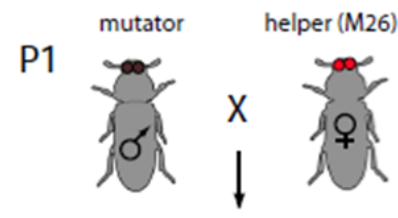

P2

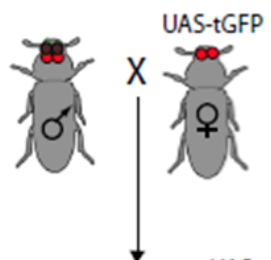

F1

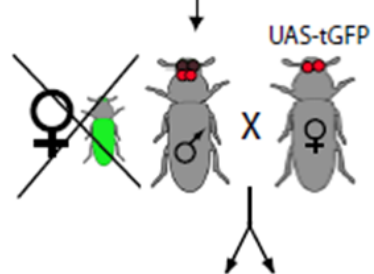

F2
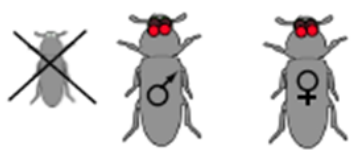

Figure 4: Crossing scheme to establish Gal4-based enhancer trap lines. Mutator line beetles carry the Gal4-piggyBac construct marked with 3xP3-v (dark eyes) homozygously on autosome " $A$ ". Beetles that have integrated the helper construct (M26 transposase) homozygously on the X-chromosome, are identified by red fluorescing eyes. In the P1 generation, the actual enhancer trap of the mutator line (Bauchbinde) is not visible, as the UAS-reporter is missing. A male mutator is crossed with a female helper. Their male progeny with red fluorescing dark eyes is collected, as they still carry one copy of the mutator transposon and now also one copy of the transposase construct. This is the generation in which the transposon can jump into a new position in the genome. To visualize a hopping event, a reporter is crossed-in by mating the male P1 progeny with virgins that carry an UAS-turboGFP construct on a different autosome " $B$ " (P2). In the F1 generation, new insertions are detected. Beetles that still show the initial enhancer trap (Bauchbinde) are removed. Only male beetles are kept for raising a population, as they have lost the X-linked transposase. Finally, these male beetles are mated again with virgin females that carry the reporter construct to establish a stock. The scheme was kindly provided by Professor Gregor Bucher, Georg-August-University Göttingen.

\subsection{Benzoquinones in beetle defensive secretions}

\subsubsection{Characteristics and molecular functions}

Defensive chemical secretions of tenebrionid beetles have broad antimicrobial function (Li et al., 2013; Prendeville and Stevens, 2002; Yezerski et al., 2007), are effective against entomopathogenic fungi (Pedrini et al., 2015) and act on parasitic helminths (Yan and Phillips, 1996). The compounds primarily responsible for the toxic and repellent effect are the $p$ benzoquinones (MBQ and $\mathrm{EBQ})$, whereas the alkenes, especially 1-pentadecene, serve as minor 
INTRODUCTION

irritants and spreading agents (Blum, 1981; Markarian et al., 1978; Peschke and Eisner, 1987; Schildknecht et al., 1964). $p$-benzoquinones have been shown to reduce cell viability and to induce DNA damage by forming adducts with DNA bases leading to mutations and DNA double strand breaks (Crespo et al., 2011; Xie et al., 2005), explaining their special cytotoxicity and function as microbial growth inhibitor. In $T$. castaneum, the defensive secretions provide an external immune defense (Joop et al., 2014). Moreover, the benzoquinones keep the food source free from microorganisms, which otherwise would compete with the beetle or make the substrate unsuitable (Sokoloff, 1974). In addition, it is reported that benzoquinones released in stress situations and under high population density function as anti-aggregation pheromones, leading beetles to leave the high-density culture and search for new food sources to colonize (Duehl et al., 2011).

\subsubsection{The need for an adequate barrier for self-protection}

When juvenile stages are exposed to high concentrations of benzoquinones produced by adult beetles, they experience numerous malformations to the point of complete lack of body structures, resulting in premature death (Roth and Howland, 1941). Adult beetles are protected from their own secretions internally and externally by cuticular linings. The secretion production takes place in a special cuticle-lined organelle, and toxic products are stored in sacs formed from invaginations of the cuticle (Happ, 1968; Roth, 1943). To prevent self-intoxication of newly hatched beetles, the secretion synthesis starts about four days after hatching, which is when the cuticle tanning process is usually completed (Unruh et al., 1998; Wirtz et al., 1978).

\subsubsection{Model for benzoquinone production in T. castaneum}

A hypothetical plan of benzoquinone production in the gland secretory cell-type 2 of T. castaneum and E. longicollis has been proposed by Happ (1968) (Figure 5). Based on thinlayer chromatography, chemical reaction tests, as well as several histochemical enzymatic activity assays, the author suggests phenolic glucosides to serve as precursors that are first transported from the cytoplasm of secretory cell $2 a$ into the vesicle of cell $2 a$. Inside, a ßglucosidase is supposed to cleave off the glucose, which may be transported back into the cytoplasm. The resulting free and harmless diphenols enter the head of the vesicular organelle. 


\section{INTRODUCTION}

In this cuticle-lined reaction chamber the final oxidation step to toxic benzoquinones seem to occur and is probably accomplished by a copper-containing phenoloxidase. The role of peroxidases, which are predicted in the efferent tubule of both type 2 and type 1 cells, remains elusive.

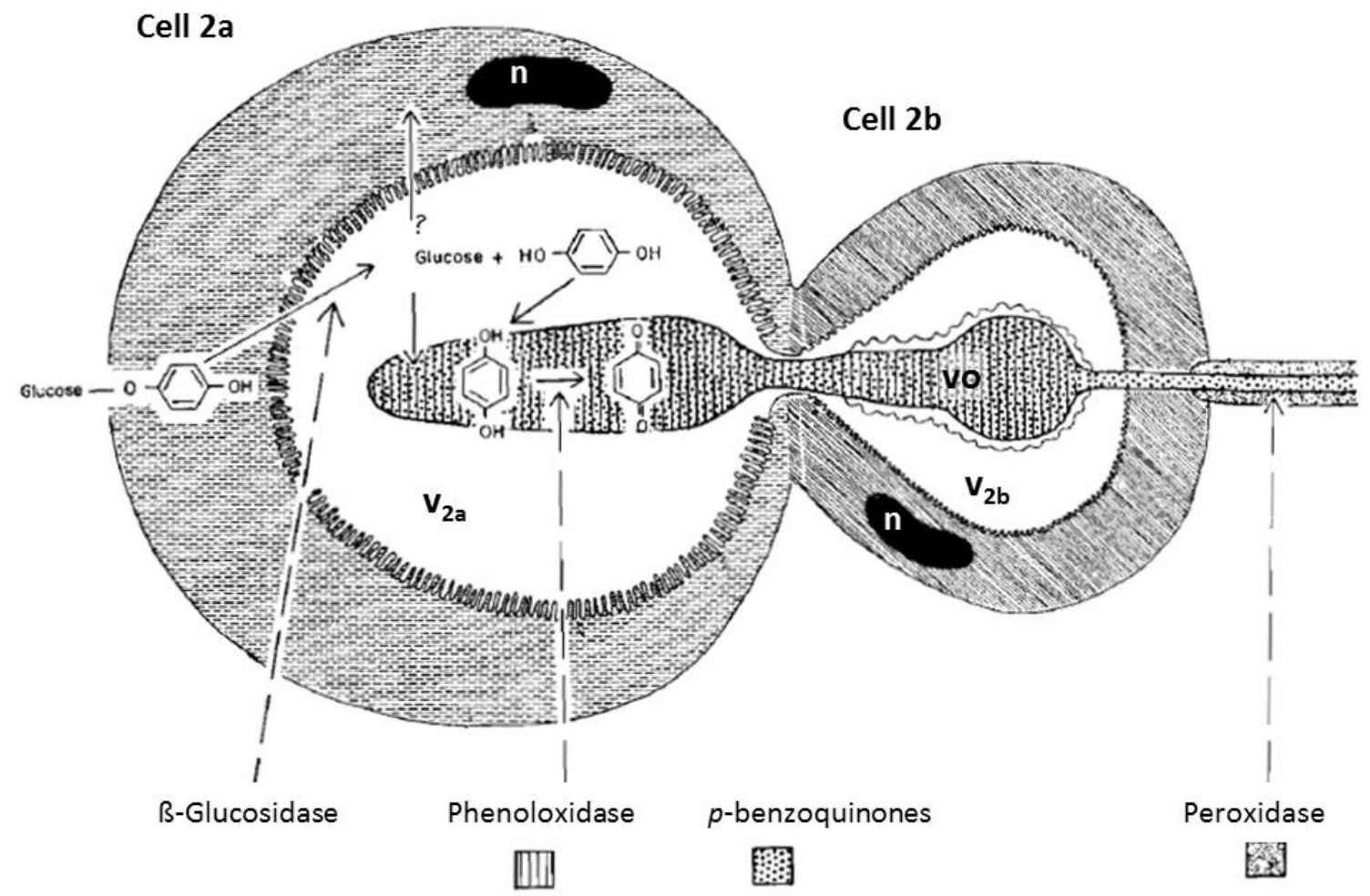

Figure 5: Hypothesized model for quinone synthesis in secretory cell-type 2 of the defensive glands of T. castaneum and $E$. longicollis (Happ, 1968, modified). For details see text. $n$ : nucleus, $\mathrm{v}_{2 \mathrm{a}}$ : vesicle of cell $2 a, v_{2 b}$ : vesicle of cell $2 b$, vo: vesicular organelle.

\subsection{Multicopper containing oxidases}

Multicopper oxidases (MCOs) are oxidizing enzymes that use copper as cofactor. Copper ions are bound in certain copper sites of the protein and are directly involved in substrate oxidation and the simultaneous reduction of molecular oxygen to water (Dittmer et al., 2004). Their number and geometric arrangement in the active site determines the reaction mechanism, by which the substrate gets oxidized. Accordingly, the MCO-family distinguishes between enzymes that contain: one copper-ion (mononuclear, e.g. amine oxidase), a coupled pair of copper-ions 
INTRODUCTION

(binuclear, e.g. tyrosinase and hemocyanin), four copper-ions (arranged in four copper sites, three of them forming a trinuclear copper cluster; e.g. laccase and ascorbate oxidase), and six copper-ions (e.g. the ferroxidase ceruloplasmin) in the active center (Solomon et al., 1996, 2008). Despite their fundamentally different reactivity with dioxygen, these enzymes have overlapping substrate specificities and can occur in the same sample (Laufer et al., 2006).

\subsubsection{Laccase 2 - a phenol-oxidizing enzyme in the insect cuticle}

The largest group of enzymes in the MCO-family is represented by laccases (Peng et al., 2014). Laccase activity is dependent on the availability of oxygen, but the enzyme typically does not need hydrogen peroxide to be fully active. Peroxidases are functionally related to laccases, but require hydrogen peroxide and are considered distinct from laccases (Baldrian, 2006; Coy et al., 2010). The substrate range for laccases is broad. Besides the oxidation of ortho- and paradiphenols to their corresponding quinones, they also oxidize other polyphenols, substituted phenols, diamines, and aromatic amines (Dittmer et al., 2004; Gorman et al., 2008). Due to the phenol-oxidizing activity, these enzymes are also designated as phenoloxidases. Confusingly, the term 'phenoloxidase' is equally used as another name for tyrosinase, another copper-containing oxidase but structurally and functionally different from laccase (see section 2.5.2). Laccases are present in bacteria, fungi, plants and insects. Roles attributed to laccases include lignin biosynthesis and leaf wound healing in plants, lignin degradation in fungi, pigment production in fungi and bacteria, and metal transport and homeostasis in bacteria (Hoegger et al., 2006; McCaig et al., 2005). Relatively little is known about the functions of laccases in insects. In $T$. castaneum, Laccase 2 (Lac2) has a well described role in the oxidation of precursors to orthoquinones used in the cuticle sclerotization and pigmentation of the beetle (Figure 6; Arakane et al., 2005, 2009). Cuticle laccases were also identified in the dipterans D. melanogaster (fruitfly; Sugumaran et al., 1992), Lucilia cuprina (sheep blowfly; Barrett, 1987), and Anopheles gambiae (malaria mosquito; He et al., 2007), in the lepidopterans Bombyx mori (silkworm; Yamazaki, 1972; Yatsu and Asano, 2009) and Manduca sexta (tobacco hornworm; Dittmer et al., 2009; Thomas et al., 1989), in hemipteran stink bugs (e.g. Riptortus pedestris, Futahashi et al., 2011), in the coleopteran pine sawyer beetle Monochamus alternatus (Niu et al., 2008), and in the hymenopteran honeybee Apis mellifera (Elias-Neto et al., 2010). Some reports also described 


\section{INTRODUCTION}

laccase-like enzymatic activity in other insect tissues, e.g. in the salivary glands of the green rice leaf hopper and the pea aphid (Hattori et al., 2010; Liang, 2006), in the gut of the termite Reticulitermes flavipes and Anopheles spp. (Coy et al., 2010; Gorman et al., 2008; Sidjanski et al., 1997), and even in the venom of the parasitoid wasp Pimpla hypochondriaca (Parkinson et al., 2001), suggesting the existence of further laccases with physiological roles completely different from cuticle tanning.

\subsubsection{Tyrosinase - the phenoloxidase required for insect innate immune response}

Another multicopper containing enzyme is tyrosinase, commonly known and in the following referred to as phenoloxidase (PO), which is in structure and sequence similar to hemocyanins and hexamerins and is therefore ranked as a member of the arthropod-hemocyanin-superfamily (Burmester, 2001). However, hemocyanin-related proteins have strikingly different functions. Whereas arthropod hemocyanins are mainly responsible for the oxygen transfer in the hemolymph, hexamerins do not contain copper and thus do not bind oxygen. Instead, they are suggested as storage proteins associated with molting cycles and nutritional conditions (Burmester, 2001; Cristino et al., 2010). PO is found in invertebrates, vertebrates, plants, and microbes, displaying different physiological functions (Lu et al., 2014). In insects and other arthropods, this enzyme is a key player in the innate immune system. With its ability to hydroxylate monophenols to $o$-diphenols and to oxidize $o$-diphenols to their corresponding $o$ quinones, PO catalyzes the first steps in the melanization pathway as part of the innate immune response to microbes and parasitoids invading into the hemolymph (Figure 6; Cerenius and Söderhäll, 2004). PO is produced as an inactive pro-enzyme (proPO) in special insect blood cells (hemocytes) and is released into the hemolymph probably by cell rupture. The presence of foreign material, e.g. parasitoid microbes, induces a complex serin protease cascade (proPO activating system), which activates the pro-enzyme by a limited proteolysis of an N-terminal peptide. After activation, the enzyme oxidizes hemolymphal DOPA and dopamine to their corresponding o-quinones, which can kill the invading microorganisms directly and contribute to melanin formation and final encapsulation of the invaders (Andersen, 2010; González-Santoyo and Córdoba-Aguilar, 2012; Lu et al., 2014). 
INTRODUCTION

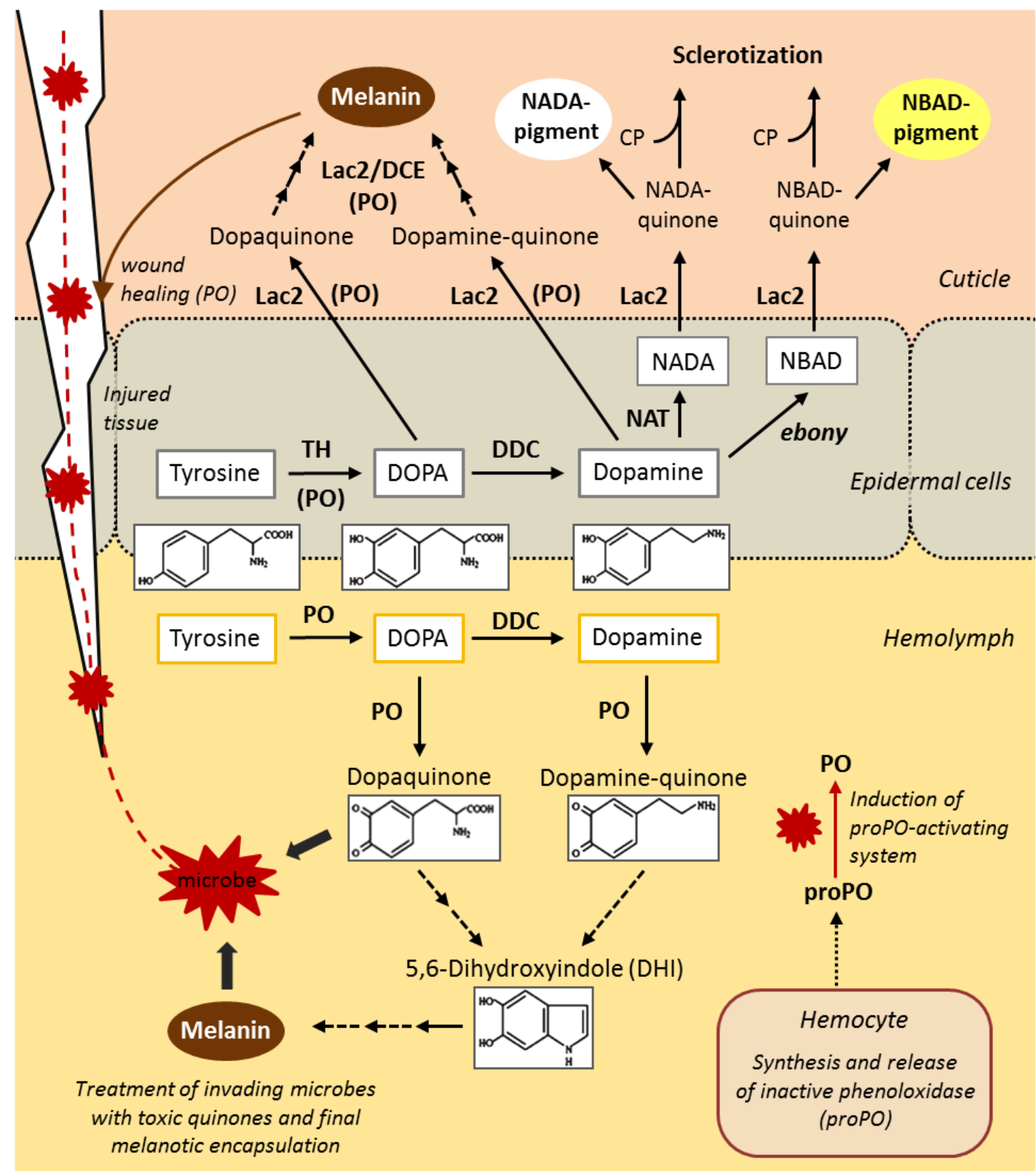

Figure 6: Roles of phenoloxidases in the beetle. In epidermal cells (gray), precursors (DOPA, Dopamine, NADA, NBAD) are produced for sclerotization and pigmentation (Melanin, NADA-pigment, NBADpigment) of the cuticle. Oxidation of precursors to corresponding o-quinones is accomplished by Laccase 2 (Lac2). In case of an injury of the tissue, melanin is produced for wound healing by phenoloxidase (PO). When a pathogen (red star) enters the hemolymph, proPO gets activated (red arrow) and starts the melanization process to encapsulate the invader. Two or more arrows in a row indicate the existence of not displayed intermediates and dashed arrows mark reaction steps that do not need enzymatic catalyzation. CP: cuticle proteins, DCE: Dopamine conversion enzyme, DDC: DOPA decarboxylase, DOPA: 3,4-dihydroxyphenylalanine, Dopamine: 3,4-dihydroxyphenethylamine, ebony: NBAD synthetase, NADA: N-acetyldopamine, NAT: N-acetyltransferase, NBAD: N-ß-alanyldopamine, TH: Tyrosine hydroxylase (after Andersen, 2010; Arakane et al., 2009; González-Santoyo and CórdobaAguilar, 2012). 


\section{INTRODUCTION}

\subsubsection{Overlapping substrate specificities of phenol-oxidizing enzymes in the beetle}

Lac2 is able to oxidize both 0 - and $p$-diphenols to their corresponding quinones, whereas PO oxidizes no other than 0 -diphenols. This overlapping substrate specificity makes both enzymes capable of catalyzing the formation of o-quinones that are needed for sclerotization and pigmentation of the exoskeleton of $T$. castaneum (Figure 6). Indeed, both phenol-oxidizing enzymes are present in the cuticle of the beetle. However, in contrast to Lac2, PO is most likely not involved in insect cuticle tanning (Arakane et al., 2005, 2009), although cuticular melanin is generated in the same pathway as hemolymphal melanin (compare Figure 6). The presence of PO in the cuticle is considered to be rather related to its functioning in wound healing and preventing microorganisms from entering the individual at the damaged site of the tissue (Andersen, 2010; Binggeli et al., 2014).

\subsection{Aims of this work}

From stink gland studies of other tenebrionid beetles we know that the special vesicular organelle is different in the two gland cell-types. With this study, I wanted to shed light onto the morphology of $T$. castaneum secretion producing cells, with a special focus on the ultrastructure of the vesicular organelle, thereby examining how the construction of gland cells contributes to the biosynthesis of secretion.

In addition, to better understand the molecular genetic basis for the protected synthesis of toxic substances, I aimed to identify further genes that are involved in stink gland development and physiology. Moreover, I planned to put special emphasis on the characterization of genes encoding Tribolium glucosidases, phenoloxidases, and peroxidases to identify those genes that fulfill the proposed functions in the hypothesized model for benzoquinone biosynthesis. 
MATERIAL AND METHODS

\section{Material and Methods}

\subsection{Beetle strains and animal rearing}

This study is based on the T. castaneum wildtype strain San Bernardino (SB), which originated from Alexander Sokoloff, California. The beetles were reared on whole grain flour at $27^{\circ} \mathrm{C}$ and $30-50 \%$ relative humidity. In gene knockdown experiments, the injected pupae were kept at $32^{\circ} \mathrm{C}$ until analysis of the adult beetles. Unless indicated otherwise, experiments were performed with beetles ten days after hatching of the imago.

\subsection{Gland cytology}

\subsubsection{Subcellular studies}

To investigate the cuticular vesicular organelle structures of the two gland cell types, gland tissues of wildtype beetles were dissected and incubated in $10 \%$ potassium hydroxide at $70^{\circ} \mathrm{C}$ for $10 \mathrm{~min}$ to detach non-cuticular cell structures. Then the remaining cuticle-containing structures of the glands were mounted in Mowiol $^{\circledR} 4-88$ and analyzed under the Axioplan2 imaging microscope.

\subsubsection{Ultrastructural studies}

Transmission electron microscopy (TEM) studies were performed in the Department for Cellular Neurobiology (Prof. Dr. Martin Göpfert), Georg-August-University Göttingen, with the help of Margret Winkler. Abdominal and prothoracic glands of wildtype beetles were dissected and incubated in 2\% PFA (Merck, Millipore KGaA, Darmstadt, Germany) and 2.5\% Glutaraldehyde (Agar Scientific, Stansted, United Kingdom) buffered in $50 \mathrm{mM}$ Na-Cacodylate pH 7.4 (Dimethylarsinsäure Natriumsalz, Merck, Millipore KGaA, Darmstadt, Germany) over night at $8^{\circ} \mathrm{C}$. The fixative was removed and the glands were rinsed two times with $50 \mathrm{mM}$ Na-Cacodylate buffer at RT before they were postfixed in $2 \% \mathrm{OsO}_{4}$ (Carl Roth $\mathrm{GmbH} \&$ Co KG, Karlsruhe, Germany) in $50 \mathrm{mM} \mathrm{Na-Cacodylate} \mathrm{buffer} \mathrm{for} 2 \mathrm{~h}$ at $8^{\circ} \mathrm{C}$. The glands were washed with ethanol (Merck Millipore KGaA, Darmstadt, Germany, 2x 30\%, 2x 50\%, 7 min each) and stained with

saturated uranyl acetate (Serva, Heidelberg, Germany) in 70\% ethanol for $30 \mathrm{~min}$. After rinsing the samples with $70 \%$ ethanol, the dehydration serial with ethanol was continued $(2 x 80 \%, 2 x$ 


\section{MATERIAL AND METHODS}

$90 \%, 2 \times 96 \%, 7$ min each; 2x 100\%, 10 min each). Subsequently, the gland tissues were transferred into propylenoxide (Merck Millipore KGaA, Darmstadt, Germany, 2x 10 min) and then stepwise into durcupan (Fluka, Buchs, Switzerland): propylenoxide/durcupan in ratio 3:1 for $1 \mathrm{~h}$, in ratio $1: 1$ for $1 \mathrm{~h}$ up to $16 \mathrm{~h}$, in ratio $1: 3$ for 1 and finally in pure durcupan for $1 \mathrm{~h}$. The tissues were embedded in a flat layer of fresh durcupan on a PTFE soaked microscope slide, covered with a coverslip and incubated for $48 \mathrm{~h}$ at $65^{\circ} \mathrm{C}$. After polymerization, the embedded glands were cut out with a scalpel and stuck on hardened durcupan blocks with super glue. After incubation for one day at $65^{\circ} \mathrm{C}$, the samples were trimmed and cut to ultrathin sections (50-80 $\mathrm{nm})$ with an ultramicrotome UltracutE from Reichert-Jung using a diamond knife. The sections were applied to copper grids (G2450 C hexagonal, Plano, Wetzlar, Germany) that have been coated with $0.3 \%$ Formvar in chloroform (Merck) and were stained with saturated uranyl acetate for $30 \mathrm{~min}$ and with lead citrate (Reynolds, 1963) for $2 \mathrm{~min}$. The gland cell's ultrastructure was observed with a Zeiss EM 902A transmission electron microscope. Microscopic pictures were taken using a slow scan CCD-camera for TEM connected to a TRS Dual Speed camera controller (Tröndle, Moorenweis, Germany).

\subsection{Origins of candidate genes}

\subsection{1 iBeetle rescreen}

After the first phase of the iBeetle screen (Schmitt-Engel et al., 2015), the iBeetle database (Dönitz et al., 2015) was searched for genes annotated with a stink gland phenotype upon RNAimediated knockdown. To reproduce and thereby confirm these phenotypes, the selected genes from the database were screened again. This time, gene fragments independent from the original iBeetle (iB) fragments, so called non-overlapping fragments (NOF), were used for the design of dsRNA to exclude that the annotated gland phenotypes were due to off-target effects of the injected dsRNA. As some genes had a rather short coding sequence, the original iB fragments covered the coding sequence to a big extent, so that for their rescreen overlapping fragments had to be used instead of NOF's. 
MATERIAL AND METHODS

\subsubsection{Gland transcriptome data}

Tribolium stink gland transcriptome data were searched for differentially expressed putative phenoloxidases and peroxidases in the gland tissues to identify candidates for the enzymatic synthesis of benzoquinones in the gland secretion. Additionally, candidate genes that were identified by other approaches (see 3.3.1 iBeetle screen and 3.3.3 Gal4 enhancer trap screen) were reviewed with respect to their expression in the gland tissues and reconsidered where necessary. RNA-sequencing and the generation of gland transcriptome data has been done by Jianwei Li and is described in Li et al. (2013).

\subsubsection{Gal4 enhancer trap screen}

In a Gal4-based enhancer trap screen that has been refined from the insertional mutagenesis screen from Trauner et al. (2009) by using a modified piggyBac transposon construct and a Gal4-mutator line and a UAS-turboGFP-reporter line, insertional enhancer trap lines were screened for turboGFP fluorescence by Elke Küster in the laboratory of Prof. Gregor Bucher. Lines showing green fluorescence in pupal abdominal and prothoracic glands were further analyzed to locate the genomic insertion sites. To this end, genomic DNA was extracted from 23 pupae or adults, digested with Bsp143I or EcoRV, self-ligated and addressed to inverse PCR (adapted from Trauner et al., 2009). The obtained DNA fragments that flanked the insertion site were sequenced and subsequently searched against $T$. castaneum genome (NCBI and $\mathrm{ftp}: / /$ ftp.bioinformatics.ksu.edu/pub/BeetleBase analyzed in Geneious ${ }^{\circledR}$ ). If insertions appeared in predicted Tribolium genes, BLAST analysis was performed to get the top Drosophila hit. For insertions that didn't match a so far predicted gene, it was assumed that they might have hit an enhancer region. For time reasons, only the directly neighbored genes up- and downstream of the possible enhancer region were considered as the respective target. Together with the hit predicted genes these candidates were analyzed further by RNAi-mediated gene knockdown to test their involvement in stink gland processes. 


\section{MATERIAL AND METHODS}

\subsection{Sequence Analysis}

\subsubsection{Identification of conserved domains}

The National Center for Biotechnology Information (NCBI, Bethesda, USA) conserved domain search (http://www.ncbi.nlm.nih.gov/Structure/cdd/wrpsb.cgi, Marchler-Bauer et al., 2015) was used to identify conserved domains in nucleotide or amino acid sequences.

\subsubsection{Prediction of subcellular location of proteins}

Different online tools were used to predict N-terminal and C-terminal sorting signals of proteins. iPSORT (http://www.ipsort.hgc.jp/, Bannai et al., 2002) and TargetP 1.1 (http://www.cbs. dtu.dk/services/TargetP/, Emanuelsson et al., 2000, 2007) were used to predict mitochondrial targeting peptides and secretory pathway signal peptides. PTS1 predictor (http://mendel.imp. ac.at/mendeljsp/sat/pts1/PTS1predictor.jsp, Neuberger et al., 2003a, 2003b) was used to search for C-terminal peroxisomal targeting signals.

\subsubsection{Phylogenetic studies}

Phylogenetic trees of peroxiredoxins and insect laccases were constructed in MrBayes 3.2 (as a Geneious $^{\circledR}$ incorporation) with standard settings according to the instructor's manual (rate matrix poisson, chain length 1.100.000, burn-in length 100.000, Ronquist et al., 2011: http:// mrbayes.sourceforge.net/mb3.2_manual.pdf, Ronquist and Huelsenbeck, 2003), on the basis of ClustalW alignments of complete amino acid sequences (Larkin et al., 2007). The NCBI accession numbers of sequences used for phylogenetic analysis of Tribolium peroxiredoxins are: Drosophila melanogaster DmDPx-4783 (NP_477510), DmDPx-5037 (NP_524387), DmDPx-4156 (NP_525002), DmPrxV (NP_650679.3), DmDPx-2540_1 (AAG47823), DmDPx-2540_2 (AAG47824), DmDPx-6005 (Q9GPQ2 on UniProt database, Consortium, 2015), Mus musculus MmPrdx1 (NP_035164), MmPrdx2 (NP_035693), MmPrdx3 (NP_031478.1), MmPrdx4 (NP_058044), MmPrdx5 (NP_036151.1), MmPrdx6 (NP_031479.1), Homo sapiens HsPrdx1_a (NP_002565), HsPrdx1_b (NP_857634.1), HsPrdx1_c (NP_857635.1), HsPrdx2 (NP_005800), HsPrdx3 (AAH08435), HsPrdx4 (NP_006397.1), HsPrdx5 (NP_036226.1), HsPrdx6 (NP_004896.1), Tribolium castaneum Tc001071 (XP_975268), Tc001700 (XP_970881.1), Tc004948 (XP_968419), Tc007642 (XP_969254.2), Tc013791 (XP_970660.2) Tc014929 (XP_970797) and for Tc012328 
the RACE confirmed nucleotide sequence translated with the translate tool from ExPASy (Gasteiger et al., 2003). For Tc12328 sequences see appendix 7.7.

\subsubsection{Analysis of DNA sequences}

Sticky end cloning was planned with geneious ${ }^{\boxplus 7.1 .5}$ (Kearse et al., 2012). For analysis of DNA sequencing results, Chromas Lite 2.1.1 (Technelysium Pty Ltd, 2013) was used to display the chromatograms. Sequence alignments were performed with MultAlin (http://multalin.toulouse. inra.fr/multalin/, Corpet, 1988) and the Basic Local Alignment Tool (BLAST ${ }^{\circledR}$ ) on NCBI (http://blast.ncbi.nlm.nih.gov/Blast.cgi, Altschul et al., 1990).

\subsection{Molecular biology}

\subsubsection{Oligonucleotides}

Oligonucleotides were ordered from Eurofins MWG Operon (Ebersberg, Germany). All used oligonucleotides are listed with their sequence and the respective Tribolium gene in the appendix (7.1).

\subsubsection{Fishing PCR}

The amplification of DNA sequences of Tribolium genes for the purpose of dsRNA synthesis or RNA probe production was performed with the Phusion ${ }^{\circledR}$ High-Fidelity DNA polymerase (Finnzymes, Thermo Fisher Scientific Germany BV \& Co KG, Braunschweig, Germany) using cDNA from Tribolium adult stink gland tissue as template. The double stranded cDNA library has been produced by a former PhD student from poly(A) mRNA following the instructions of the SMART ${ }^{T M}$ PCR cDNA Synthesis Kit (Clontech, Saint-Germain-en-Laye, France; Li, 2013 section 2.7) and was adopted for these studies. The Eppendorf Mastercycler personal (Eppendorf AG, Hamburg, Germany) was used as PCR cycler and programs were set up according to the manufacturer's instructions.

\subsubsection{Rapid amplification of cDNA ends (RACE)}

The 5'- and 3'-RACE-ready cDNA libraries were synthesized from adult beetle poly(A) mRNA with the SMART ${ }^{T M}$ RACE cDNA Amplification Kit (Clontech, Saint-Germain-en-Laye, France) and the 


\section{MATERIAL AND METHODS}

Advantage ${ }^{\circledR} 2$ PCR Kit (Clontech, Saint-Germain-en-Laye, France) was used to perform the RACE subsequently, according to the specifications in the user manuals.

\subsubsection{Cloning}

Molecular cloning was carried out following standard protocols (Green and Sambrook, 2012) or according to respective user manuals. Briefly, DNA sequences were amplified by PCR (see 3.5.2), purified from agarose gels via the NucleoSpin ${ }^{\circledR}$ Gel and PCR Clean-up Kit (Macherey-Nagel, Düren, Germany), and ligated by T4 DNA ligase (Fermentas GmbH, St. Leon-Rot, Germany, and New England Biolabs, Frankfurt a.M., Germany). DNA templates for later dsRNA- and RNA probe synthesis were ligated into pJET1.2/blunt vector. For ligation of RACE-PCR products into $\mathrm{PCR}{ }^{\otimes} \|$ vector, the TA Cloning ${ }^{\circledR}$ Kit Dual Promoter (Invitrogen GmbH, Karlsruhe, Germany) was used. Heat shock transformation with chemically competent DH5 $\alpha$ E. coli was performed and colony PCR was used to verify positive clones. Plasmid DNA was extracted using the NucleoSpin ${ }^{\circledR}$ Plasmid Kit (Macherey-Nagel, Düren, Germany) and sent to the sequencing service LGC genomics (Berlin, Germany).

\subsection{RNA interference-mediated gene knockdown}

\subsubsection{Synthesis of double-stranded RNA}

Double-stranded RNAs (dsRNAs) for RNA interference (RNAi) experiments were either ordered from Eupheria Biotech GmbH (Dresden, Germany) or self-made. In the latter case the E-RNAi web service of the German cancer research center (Horn and Boutros, 2010) was used to define fragments for dsRNA synthesis with no off-targets. The fragments were cloned (see 3.5.4 and 7.1 for oligonucleotides) and the in vitro transcription was performed with the MEGAscript ${ }^{\circledR}$ T7 Kit from Ambion ${ }^{\circledast}$ (ordered from Life Technologies GmbH, Darmstadt, Germany, Cat\#: AM1334) using the purified PCR product of the respective gene fragment with added T7-RNA Polymerase promoter sites as template DNA. The synthesized dsRNAs were resuspended and diluted in injection buffer (10x stock: $14 \mathrm{mM} \mathrm{NaCl}, 0.7 \mathrm{mM} \mathrm{Na} 2 \mathrm{HPO}_{4} \cdot 2 \mathrm{H}_{2} \mathrm{O}, 0.3 \mathrm{mM} \mathrm{KH}_{2} \mathrm{PO}_{4}, 40 \mathrm{mM} \mathrm{KCl}$ ) and stored at $-20^{\circ} \mathrm{C}$ 
MATERIAL AND METHODS

\subsubsection{Injection of dsRNA}

Injections were performed at mid-pupal stage in male and female animals. Pupae were incubated on ice for $5 \mathrm{~min}$ and lined up on adhesive tape on a microscope slide. Injection needles were pulled with Micropipette Puller Model P-2000 (Sutter Instruments, Novato, USA) using borosilicate glass capillaries (length: $100 \mathrm{~mm}$, outside-diameter: $1 \mathrm{~mm}$, wall thickness: $0.21 \mathrm{~mm}$, Heinemann Labortechnik $\mathrm{GmbH}$, Duderstadt, Germany) and loaded with dsRNA by the use of Femtotips $^{\circledR}$ (Eppendorf, Hamburg, Germany). For semiautomatic injections, a FemtoJet ${ }^{\circledR}$ express microinjector (Eppendorf, Hamburg, Germany) was used in combination with a micromanipulator (M1, Helmut Saur Laborbedarf, Reutlingen, Germany). In the iBeetle rescreen, dsRNAs were injected at a concentration of $2 \mu \mathrm{g} / \mu \mathrm{l}$. For genes whose stink gland phenotypes could not be reproduced injections were first tried with higher dsRNA concentration $(3 \mu \mathrm{g} / \mu \mathrm{l})$ and, if necessary, repeated in larvae. For candidate genes selected from the gland transcriptome library and the Gal4 enhancer trap screen, dsRNA concentrations of 1 $\mu \mathrm{g} / \mu \mathrm{l}$ were used.

\subsection{Localization of gene expression in gland tissue}

\subsubsection{Synthesis of digoxigenin (DIG) -labeled RNA probes}

RNA probes were synthesized from purified PCR products (with added T3- and T7-RNA Polymerase promoter sites) by using the DIG RNA Labeling Mix, T3- and T7-RNA Polymerase, and Protector RNase Inhibitor (Roche Applied Science, Mannheim, Germany, Cat\#: 11277073910, Cat\#: 11031163001, Cat\#: 10881767001 and Cat\#: 03335399001, respectively) in an in vitro transcription reaction according to the user manual. The DIG-labeled RNA probes were solved in RNA resuspension buffer (50\% Formamide, 0.1\% Tween20, 5x SSC pH 4.5, 20 $\mu \mathrm{g} / \mathrm{ml}$ heparin) and stored at $-20^{\circ} \mathrm{C}$ until use.

\subsubsection{Gland whole mount in situ hybridization}

Glands were dissected out of the adult beetles leaving a small piece of the exoskeleton attached to them and were pinned to the sylgard bottom (Sylgard 184, Cat\#: SYLG184, World Precision Instruments, Berlin, Germany) of a 24 well-plate (about four abdominal and four prothoracic

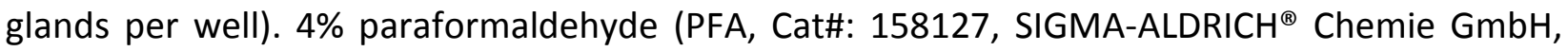




\section{MATERIAL AND METHODS}

Munich, Germany) in phosphate-buffered saline (1x PBS, 10x stock: $1.37 \mathrm{M} \mathrm{NaCl}, 27 \mathrm{mM} \mathrm{KCl}, 20$ $\mathrm{mM} \mathrm{KH}_{2} \mathrm{PO}_{4}, 100 \mathrm{mM} \mathrm{Na}_{2} \mathrm{HPO}_{4}, \mathrm{pH}$ 7.4) was added and the glands were incubated for $2.5 \mathrm{~h}$ at $8^{\circ} \mathrm{C}$. After rinsing and washing for $10 \mathrm{~min}$ in PBT (1x PBS with $0.03 \%$ TritonX-100), $5 \mu \mathrm{g} / \mathrm{ml}$ Proteinase $\mathrm{K}$ in PBT was added for $5 \mathrm{~min}$. The solution was removed and the glands were rinsed and washed in PBT for 10 min. 4\% PFA was used to postfix the gland tissues for 30 min at room temperature (RT). To remove the fixative, the tissues were rinsed and washed with PBT for 10 min. Then the glands were washed for 5 min in pre-warmed $50 \%$ hybridization-buffer (Hybbuffer: 50\% Formamide, 5x SSC pH 5.5, 0.03\% TritonX-100, $100 \mu \mathrm{g} / \mathrm{ml}$ salmon sperm DNA, $100 \mu \mathrm{g} / \mathrm{ml}$ heparin, $100 \mu \mathrm{g} / \mathrm{ml}$ Yeast RNA, in PBT) at $60^{\circ} \mathrm{C}$ and after this incubated in Hyb-buffer for $3 \mathrm{~h}$ at $60^{\circ} \mathrm{C}$. In the meantime, RNA probes were diluted in Hyb-buffer to $50 \mathrm{ng} / \mathrm{ml}$, heated to $95^{\circ} \mathrm{C}$ for $2 \mathrm{~min}$, and immediately chilled on ice for $10 \mathrm{~min}$. At the end of the $3 \mathrm{~h}$ incubation time, the prepared probes were added to the glands $\left(1 \mathrm{ml}\right.$ per well) and incubated over night at $60^{\circ} \mathrm{C}$. On the next day, the hybridization buffer was removed and the gland tissues were rinsed and washed for 20 min in pre-warmed $2 x$ SSC (20x stock: $3 \mathrm{M} \mathrm{NaCl}, 300 \mathrm{mM}$ sodium citrate, pH 5.5 adjusted with citric acid) at $65^{\circ} \mathrm{C}$. After this, the tissues were incubated in washing buffer $(50 \%$ Formamide, $2 \times \mathrm{SSC}, 0.03 \%$ TritonX-100) for $45 \mathrm{~min}$ at $65^{\circ} \mathrm{C}$, then for $30 \mathrm{~min}$ in $50 \%$ washing buffer-TBST (TBS with $0.03 \%$ TritonX-100) at $65^{\circ} \mathrm{C}$, for $15 \mathrm{~min}$ in TBST at $65^{\circ} \mathrm{C}$, and finally for $15 \mathrm{~min}$ in TBST at RT. Subsequently, the gland tissues were blocked in blocking solution (Blocking Reagent, Cat\#: 11096176001, Roche Applied Science, Mannheim, Germany, 1:10 in TBST) for $2-4 \mathrm{~h}$ at $8^{\circ} \mathrm{C}$. The blocking solution was removed and anti-DIG-AP antibody (AntiDigoxigenin-AP, Fab fragments, Cat\#: 11093274910, Roche Applied Science, Mannheim, Germany, diluted 1:1000 in blocking solution) as well as Hoechst 33342 (bisBenzimide H 33342 trihydrochloride, Cat\#: B2261, SIGMA-ALDRICH ${ }^{\circledR}$ Chemie GmbH, Munich, Germany, $1 \mathrm{mg} / \mathrm{ml}$ stock, 1:1000) were added and incubated over night at $8^{\circ} \mathrm{C}$. On the next day, the antibody solution was removed and the tissues were washed four times in TBST for 10 min each, followed by 5 min washing in detection buffer $\left(100 \mathrm{mM}\right.$ Tris- $\mathrm{HCl}, 0.1 \mathrm{M} \mathrm{NaCl}, 0.05 \mathrm{M} \mathrm{MgCl}_{2}, \mathrm{pH} 9.5,0.03 \%$ TritonX-100). The detection buffer was removed and the staining solution ( $1 \mathrm{ml}$ detection buffer + 4.5 $\mu$ I NBT (Nitrotetrazolium Blue chloride, Cat\#: 4421.3, Carl Roth GmbH \& Co KG, Karlsruhe, Germany, $50 \mathrm{mg} / \mathrm{ml}$ in $70 \% \mathrm{DMF}),+3.5 \mu \mathrm{l} \mathrm{BCIP}{ }^{\circledR}$ (5-Bromo-4-chloro-3-indolyl phosphate 
disodium salt, Cat\#: B6149, SIGMA-ALDRICH ${ }^{\circledR}$ Chemie $\mathrm{GmbH}$, Munich, Germany, $50 \mathrm{mg} / \mathrm{ml}$ in $100 \%$ DMF)), was added to the glands. They were incubated in the dark for 30-60 min at RT. The staining reaction was stopped by rinsing two times with TBST, washing two times in TBST for 10 min each and a final wash for $10 \mathrm{~min}$ in TBS (Tris-buffered saline: $100 \mathrm{mM}$ Tris HCl pH 7.5, 150 $\mathrm{mM} \mathrm{NaCl}$ ). The glands were transferred into a drop of Mowiol ${ }^{\circledR}$ 4-88 (Cat\#: 81381-50G, SIGMAALDRICH $^{\circledR}$ Chemie $\mathrm{GmbH}$, Munich, Germany) on a microscope slide and the remains of the exoskeleton were removed. Finally, the glands were embedded in fresh Mowiol ${ }^{\circledR} 4-88$ on a new slide, hardened over night, and stored at $8^{\circ} \mathrm{C}$ until microscopic observation.

\subsection{Gland whole mount immunohistochemistry}

Glands were dissected out of the adult beetles leaving a small piece of the exoskeleton attached to them and were pinned to the sylgard bottom (Sylgard 184, Cat\#: SYLG184, World Precision Instruments, Berlin, Germany) of a 24 well-plate (ca. four abdominal and four prothoracic glands per well). The gland tissues were fixated in $4 \%$ PFA in PBS for $2 \mathrm{~h}$ at $8^{\circ} \mathrm{C}$. The fixative was removed and the glands were rinsed and three times washed for 20 min each in PBST (1x PBS with $0.1 \%$ Triton X-100). After $1 \mathrm{~h}$ incubation at RT in blocking buffer (5\% goat serum, Cat\#: G9023, SIGMA-ALDRICH ${ }^{\circledR}$ Chemie GmbH, Munich, Germany; 1\% Albumin Fraktion V, Cat\#: 8076, Carl Roth GmbH \& Co KG, Karlsruhe, Germany; in PBST), the primary antibody (polyclonal rabbit antiserum and purified antibodies of M. sexta Lac2 (Dittmer et al., 2009) or polyclonal rabbit antiserum of An. gambiae Lac2, both kindly provided by the group of Prof. Dr. Michael Kanost, Kansas State University, USA) was diluted 1:4000 in fresh blocking buffer, added to the glands, and incubated over night at $8^{\circ} \mathrm{C}$. On the next day, the gland tissues were washed four times in PBS at RT before they were incubated with the secondary antibody (goat Anti-rabbit IgG H\&L AP, Cat\#: ab97048, Abcam, Cambridge, United Kingdom, 1:1000 in blocking buffer) for $2 \mathrm{~h}$ at RT. After two washes in PBST and a wash in PBS for 20 min each, the glands were incubated for 5 min in detection buffer (100 mM Tris- $\mathrm{HCl} \mathrm{pH} \mathrm{9.5,} 0.1 \mathrm{M} \mathrm{NaCl}, 0.05 \mathrm{M} \mathrm{MgCl}_{2}$ ). The buffer was removed, the staining solution $\left(4.5 \mu \mathrm{lNBT}\right.$ and $3.5 \mu \mathrm{IBCI}{ }^{\circledast}$ per $\mathrm{ml}$ detection buffer, see section 2.7) was added to the glands, and the incubation took place in the dark at RT. The color reaction was stopped after 5-20 min by rinsing two times with PBS, washing two times for 20 min with 


\section{MATERIAL AND METHODS}

PBST, and a final wash in PBS for $20 \mathrm{~min}$. Then the glands were transferred into a drop of Mowiol $^{\circledR}$ 4-88 (Cat\#: 81381-50G, SIGMA-ALDRICH ${ }^{\circledR}$ Chemie GmbH, Munich, Germany) on a microscope slide and the remains of the exoskeleton were removed. Finally, the glands were embedded in fresh Mowiol $^{\circledR}$ 4-88 on a new slide, hardened over night, and stored at $8^{\circ} \mathrm{C}$ until microscopic analysis.

\subsection{Semi-quantitative gas chromatography-mass spectrometry analysis of gland volatiles}

Gas chromatography-mass spectrometry (GC-MS) analysis was done separately for abdominal and prothoracic glands of wildtype beetles and knockdown situations. To confirm stink gland phenotypes identified in the iBeetle rescreen, the secretions of one male and one female beetle were mixed and analyzed, whereas for candidates from the enhancer trap screen and the transcriptome data analysis three beetles of the same gender were used in one measurement, allowing to identify gender specific phenotypes. The glands were dissected ten days after hatching and crushed in $50 \mu \mathrm{l}$ (one beetle) or $100 \mu$ l (three beetles) methanol (SupraSolv ${ }^{\circledR}$ ECD and FID, Cat\#: 106011, Merck Millipore KGaA, Darmstadt, Germany). The samples were stored at $-20^{\circ} \mathrm{C}$ and measured within $48 \mathrm{~h}$.

The GC-MS system composed of a gas chromatograph (6890N Network GC System, Agilent Technologies, Santa Clara, USA), and a mass spectrometer (5973 Network Mass Selective Detector, Agilent Technologies, Santa Clara, USA) connected to a MultiPurposeSampler (MPS, Gerstel, Mülheim, Germany). Detailed technical parameters are described in Li (2013) section 2.11. Data were collected and analyzed by the software MSD ChemStation D.02.00.275 (Agilent Technologies, Santa Clara, USA). The volatile secretion compounds were identified with the NIST 2008 and Wiley $9^{\text {th }}$ edition databases (National Institute for Standards and Technology, Gaitherburg, USA; Wiley, Hoboken, USA). Microsoft Excel was used to display comparative chromatograms and to do the calculations for a semi-quantitative analysis of volatile gland secretion substances in wildtype and knockdown situations. To this end, the mean values of abundances of wildtype beetle gland chemicals (buffer injected) were determined and set as $100 \%$. Referring to the respective wildtype mean, alterations of secretion substances in 
MATERIAL AND METHODS

knockdown glands were calculated in percent, in which values $>100 \%$ indicate an increase of the respective substance in the knockdown glands compared to the wildtype and values $<100 \%$ signify a corresponding reduction. Analyzed gland secretion volatiles were the benzoquinones 2methyl-1,4-benzoquinone and 2-ethyl-1,4-benzoquinone as well as the alkenes 1-pentadecene and 1-heptadecene.

\subsection{Enzymatic activity assays}

\subsubsection{Substrates}

Two colorimetric assays were set up, using either L-DOPA - a beetle endogenous typical laccase substrate (3,4-Dihydroxy-L-phenylalanine, Cat\#: D9628, SIGMA-ALDRICH ${ }^{\circledR}$ Chemie GmbH, Munich, Germany; Mason, 1947; Terwilliger and Ryan, 2006) or the artificial substrate ABTS (2,2'-Azino-bis(3-ethylbenzothiazoline-6-sulfonic acid) diammonium salt, Cat\#: A9941, SIGMA$\mathrm{ALDRICH}^{\circledR}$ Chemie GmbH, Munich, Germany), which is suitable to detect phenoloxidase activity like L-DOPA, but can also be used to demonstrate active peroxidases in the presence of hydrogen peroxide (Collins et al., 1998; Lonergan et al., 1997). L-DOPA was buffered in $100 \mathrm{mM}$ potassium phosphate pH 7 (Liang, 2006) and ABTS in 100 mM sodium acetate pH 4.5 (Gorman et al., 2012). Both were used in a final concentration of $5 \mathrm{mM}$, if not indicated otherwise. For ABTS assays on tissue the reactions were started by adding hydrogen peroxide to final concentration of $0.006 \%$.

\subsubsection{Activity assays with gland tissue}

To test wildtype and RNAi knockdown stink gland tissues for laccase and peroxidase activity, reactions were carried out in 96 -well plates in a total volume of $100 \mu$ l each. Per reaction, the glands of two beetles were dissected freshly, separated by gender and gland type, and incubated in the dark at RT in $100 \mu \mathrm{l} \mathrm{L-DOPA}$ substrate solution, $100 \mu \mathrm{l}$ ABTS solution or $98 \mu \mathrm{l}$ ABTS solution plus $2 \mu \mathrm{l}$ of $0.3 \% \mathrm{H}_{2} \mathrm{O}_{2}$. After $60 \mathrm{~min}$, the formed products were detected by measuring the absorbance with a NanoDrop ${ }^{\circledR}$ spectrophotometer (ND-1000, software V3.7.1, Thermo Fisher Scientific Germany BV \& Co KG, Braunschweig, Germany) at $475 \mathrm{~nm}$ for dopachrome (Gorman et al., 2012) and $420 \mathrm{~nm}$ for the ABTS cation radical (Collins et al., 1998; 


\section{MATERIAL AND METHODS}

Eichlerová et al., 2012). To account for autoxidation of the substrates, reactions without gland material were included as blanks for the L-DOPA experiments. As the addition of gland material to the ABTS solution directly led to a reduction of $A_{420 \mathrm{~nm}}$ before the reaction was started, reactions that contained the substrate solution and gland material but no hydrogen peroxide served as blank.

To observe the product formation over a time period of $60 \mathrm{~min}$, the ABTS assay was also performed in $1 \mathrm{ml}$ volumes $\left(980 \mu \mathrm{ABTS}\right.$ solution plus $\left.20 \mu \mathrm{l} 0.3 \% \mathrm{H}_{2} \mathrm{O}_{2}\right)$ using the abdominal or prothoracic glands of four male and four female beetles per reaction. At time points 0 min (start of the reaction), $1 \mathrm{~min}, 5 \mathrm{~min}, 10 \mathrm{~min}, 20 \mathrm{~min}, 30 \mathrm{~min}, 40 \mathrm{~min}, 50 \mathrm{~min}$ and $60 \mathrm{~min}$ the absorbance was measured in cuvettes with a UV-Vis spectrophotometer (Uvikon940, Kontron Instruments, now tresser instruments, Rossdorf, Germany) at $420 \mathrm{~nm}$. To identify in which type of gland cells and in which subcellular structures the detected enzymatic activity is located, microscopic pictures were taken from gland tissues that were incubated for $60-90 \mathrm{~min}$ in LDOPA and ABTS solution, respectively.

\subsubsection{DOPA assay with gland secretions}

To test whether an active laccase also occurs in the gland secretions, the L-DOPA assay in the 96-well plate (3.10.2) was repeated, but instead of incubating whole tissues, the gland reservoirs were smashed to release the secretions into the substrate solution and any gland tissue was directly removed from it. Per reaction the abdominal gland content of five beetles was added to $100 \mu \mathrm{I}$ DOPA solution and incubated for $60 \mathrm{~min}$ at RT in the dark. Subsequently, the formation of dopachrome was detected by measuring the absorbance at $475 \mathrm{~nm}$ with a NanoDrop ${ }^{\circledR}$ spectrophotometer.

\subsubsection{ABTS assay with purified Lac2}

$0.05 \mathrm{mM}$ ABTS buffered in $0.1 \mathrm{M}$ sodium acetate $\mathrm{pH} 5$ was mixed with hydrogen peroxide to final concentrations of $25 \mathrm{mM}(0.075 \%), 2.5 \mathrm{mM}(0.0075 \%)$ and $0.25 \mathrm{mM}(0.00075 \%)$, respectively. $0.5 \mu \mathrm{g}$ of purified recombinant Lac2A or Lac2B was added to start the reaction. As blank control, one reaction per Laccase variant contained no hydrogen peroxide. To check for an autoxidation of ABTS in its buffer, the assay buffer (containing the ABTS) was measured with no, 
$0.075 \%, 0.0075 \%$, and $0.00075 \% \mathrm{H}_{2} \mathrm{O}_{2}$ without enzyme. Final volume for each reaction was $200 \mu \mathrm{l}$. The measurement was performed in a microplate reader at $32^{\circ} \mathrm{C}$. The absorbance at $414 \mathrm{~nm}$ was recorded every $30 \mathrm{~s}$ for $15 \mathrm{~min}$ and the activity was calculated as median absorbance increase per minute.

\subsection{Photo imaging and editing}

Pictures of gland cells after DOPA assays, gland in situ hybridization and gland immunohistochemistry were taken at a Axioplan2 imaging microscope (Carl Zeiss AG, Oberkochen, Germany) equipped with a intas camera and a Qimaging 32-100"B-358 RGB slider. Dissected abdominal and prothoracic stink glands of wildtype and RNAi knockdown beetles were recorded with a Leica MZ16 FA stereomicroscope connected to a Qimaging camera. Photographs were edited in terms of brightness and contrast using CorelPHOTO-PAINT ${ }^{\circledR} \mathrm{X} 4$ and schemes were

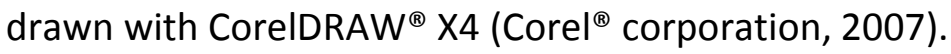

\subsection{Databases}

Tribolium gene annotations were taken from the iBeetle Genome Browser versions Tcas 4.0 and 5.2, available at http://bioinf.uni-greifswald.de/gb2/gbrowse/tcas4/ and http://bioinf.unigreifswald.de/gb2/gbrowse/tcas5/, respectively. The iBeetle-Base was searched for stink gland affecting genes (Dönitz et al., 2015; http://ibeetle-base.uni-goettingen.de/). The identification

of potential T. castaneum peroxidases was supported by PeroxiBase (Fawal et al., 2013, http://peroxibase.toulouse.inra.fr/). 

RESULTS

\section{Results}

\subsection{Gland cytology}

\subsubsection{Structure of secretion producing gland cells}

To clarify the morphology of $T$. castaneum secretory cells and their special vesicular organelles, dissected gland tissues were observed under the microscope either untreated or treated with potassium hydroxide $(\mathrm{KOH})$ to remove all non-cuticular cell structures (Figure 7).
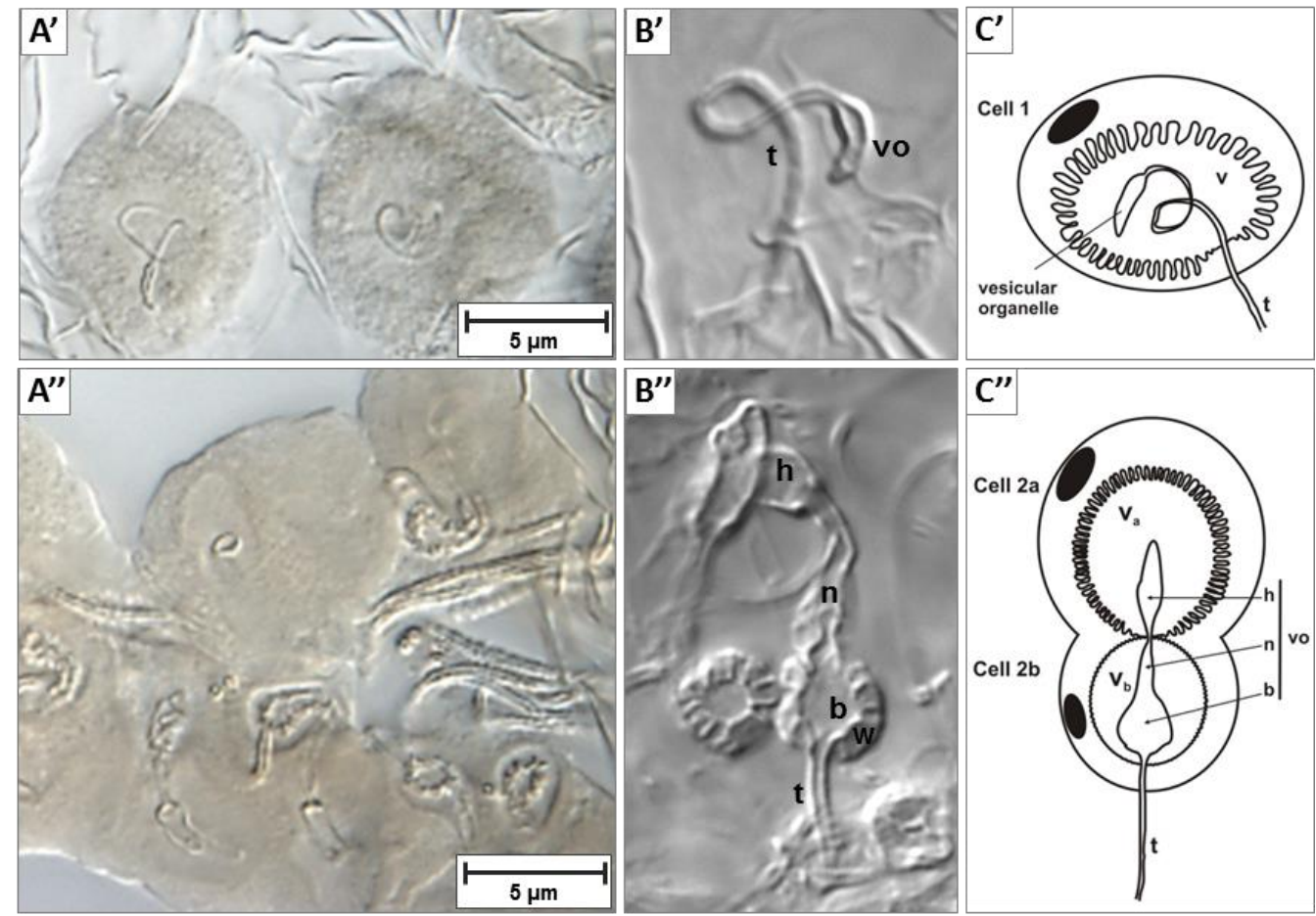

Figure 7: Morphological studies of secretory cells in the odoriferous glands of $T$. castaneum. The upper panels $A^{\prime} B^{\prime} C^{\prime}$ display untreated type 1 cells, a $\mathrm{KOH}$ released vesicular organelle of cell-type 1 and a schematic representation, respectively. The lower panels $A^{\prime \prime} B^{\prime \prime} C^{\prime \prime}$ depict the corresponding findings for secretory cell-type 2 . The schemes in panels $C^{\prime}$ and $C^{\prime \prime}$ are based on own observations and the studies of Roth (1943). For details see text. Black-filled ellipse: nucleus, b: bulb, h: head, n: neck, $r$ : reservoir, t: tubule, v: vesicle, vo: vesicular organelle, w: wall.

Coinciding with the findings of Roth (1943), cell-type 1 appears as single cells or in some cases as paired cells on the surface of the reservoir. The organelle in the type 1 cell vesicle consists of a head and a looped tubule that ends in the gland reservoir (Figure $7 B^{\prime}+C^{\prime}$ ). These observations are equal to the studies of Roth (1943), but clearly differ from the scheme in Happ (1968), 


\section{RESULTS}

where first, the organelle is shown subdivided in head and body, and second, most of the efferent tubule runs through an extra tubule carrying cell. In this study such an additional attached cell could not be observed for $T$. castaneum. The structure of the second gland celltype is more complex but found similar to the descriptions of Happ (1968). Actually, cell-type 2 is a fusion of two cells, $2 a$ and $2 b$. Both harbor a vesicle, $v_{a}$ and $v_{b}$, respectively, but share one cuticular organelle that is composed of a head, a corrugated neck, a bulb lined by an intensely folded wall, and a tubule that connects the lumen of the bulb with the reservoir (Figure $\left.7 \mathrm{~B}^{\prime \prime}+\mathrm{C}^{\prime \prime}\right)$. Whereas the head of the organelle is completely enclosed by the vesicle of cell $2 a$, neck and bulb reside in vesicle $2 b$ (Figure $\left.7 A^{\prime \prime}+C^{\prime \prime}\right)$.

\subsubsection{Ultrastructural studies of gland secretory cells}

To examine the fine structure of the vesicular organelle, transmission electron microscopy (TEM) was performed on ultrathin sections of gland tissue. TEM pictures in Figure 8 and Figure 9 display sections through different bunches of gland secretory type 2 cells. Note that due to the cutting planes not all described structures can be found in every section depicted here. In general, the cell aggregates are accompanied by tracheae and tubules and are framed by a basal membrane (Figure $8 \mathrm{~A}$, Figure $9 \mathrm{~A}-\mathrm{C}$ ). Cell membranes confining single secretory cells inside the bundle were hard to identify. One was found partly lining a $2 \mathrm{~b}$ cell (Figure 8C). However, membranes constricting tubule-carrying cells were identified easily (Figure 9B+C). Cross-sections of tubules revealed that their lumen is lined by three structures: an inner membrane, a thicker layer in the middle and an outer membrane (Figure 9B). The density and composition of secretory cell-type 2 cytoplasm varies depending on cutting plane but is generally filled with granulae of variable density and numerous mitochondria (Figure 8A, Figure 9A). Vesicles of celltype $2 \mathrm{a}$ are very prominent through their size (Figure 8A, Figure 9A). They are lined by a membrane that is surrounded by a thick layer of condensed material, referred to as wall. From this membrane, microvilli-like structures arise and head towards the center of the vesicle (Figure $8 A+B$, Figure $9 A+D$ ). The microvilli are filled and appear mostly singly. Sometimes, the linings of two or very seldom three are fused together. In comparison to the vesicle $2 a$ cells, the cell $2 \mathrm{~b}$ vesicle is much smaller and lacks the thick surrounding wall (Figure $8 \mathrm{C}$ ). 

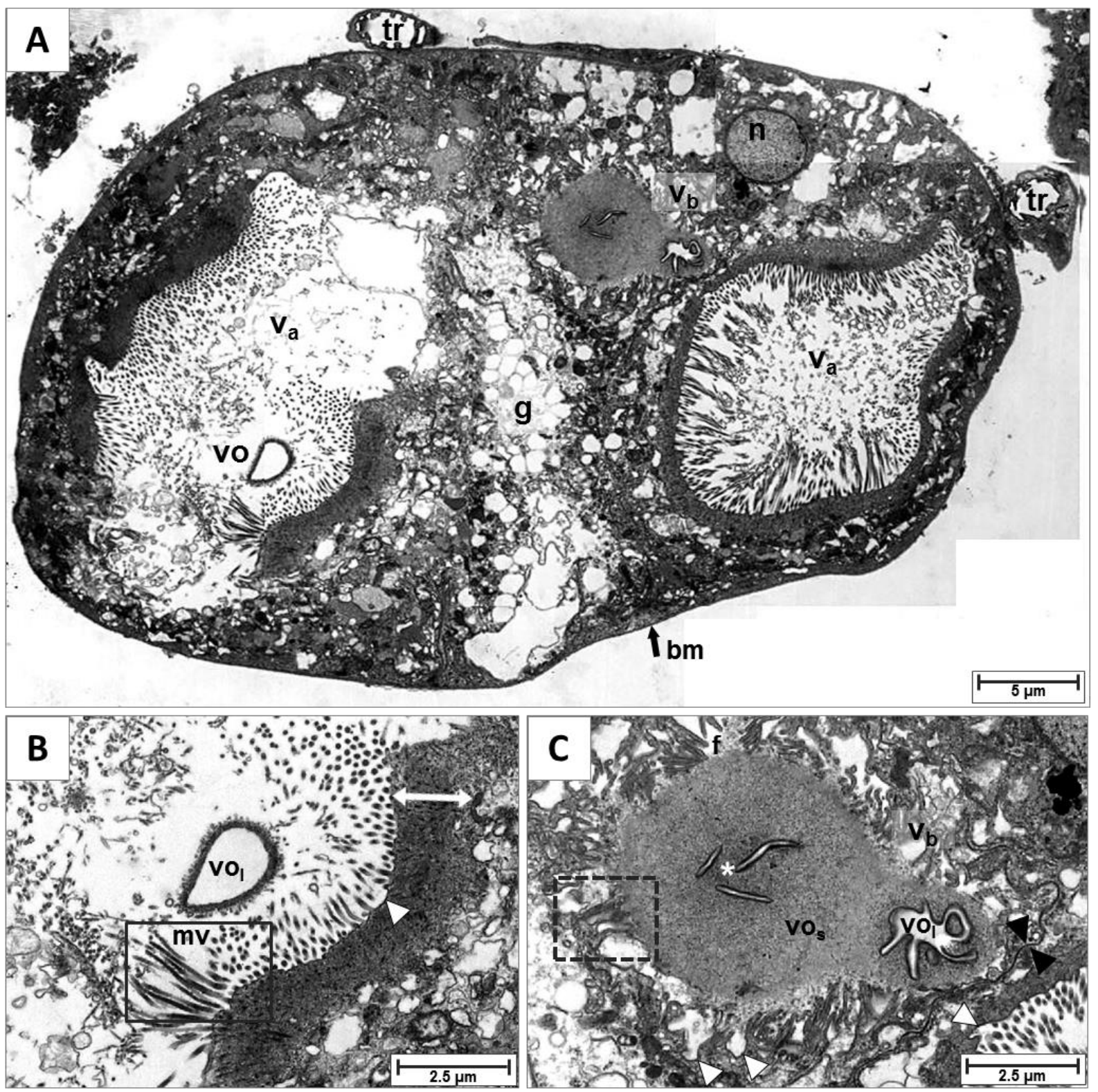

Figure 8: Ultrastructural differences between vesicles of cell-type $\mathbf{2 a}$ and $\mathbf{2 b}$. A Overview. The section shows at least two abdominal secretory type 2 cells whose cytoplasms are dense and filled with granulae. The cell bundle is constricted by a basal membrane (bm) and crossed by tracheae (tr). Note that differences in brightness and small cloudy zones are due to manual stitching of multiple single pictures. B Close up of the vesicle in type $2 \mathrm{a}$ cell $\left(\mathrm{v}_{\mathrm{a}}\right)$ harboring a vesicular organelle (vo, cross-sectioned). The black-lined box highlights microvilli-like structures $(\mathrm{mv})$ that arise from a thick vesicle lining wall (white double arrow) and appear either as stripes or as dots, depending on their growth direction and the cutting angle. C Close up of the vesicle of secretory cell type $2 b\left(v_{b}\right)$. The vesicular organelle is cut longitudinal at its outer edge. The white asterisk marks structures that will outgrow to the vesicular organelle in deeper cutting planes. The dashed black box highlights filled filaments that dock to the vesicular organelle. bm: basal membrane, f: filament, mv: microvilli-like structure, $n$ : nucleus, tr: trachea, $\mathrm{v}_{\mathrm{a}}$ : vesicle of $2 \mathrm{a}$ cell, $\mathrm{v}_{\mathrm{b}}$ : vesicle of $2 \mathrm{~b}$ cell, vo: vesicular organelle, vol: lumen of vesicular organelle, $\mathrm{vo}_{\mathrm{s}}$ : surface of vesicular organelle, $\nabla$ vesicle membrane, cell membrane. 

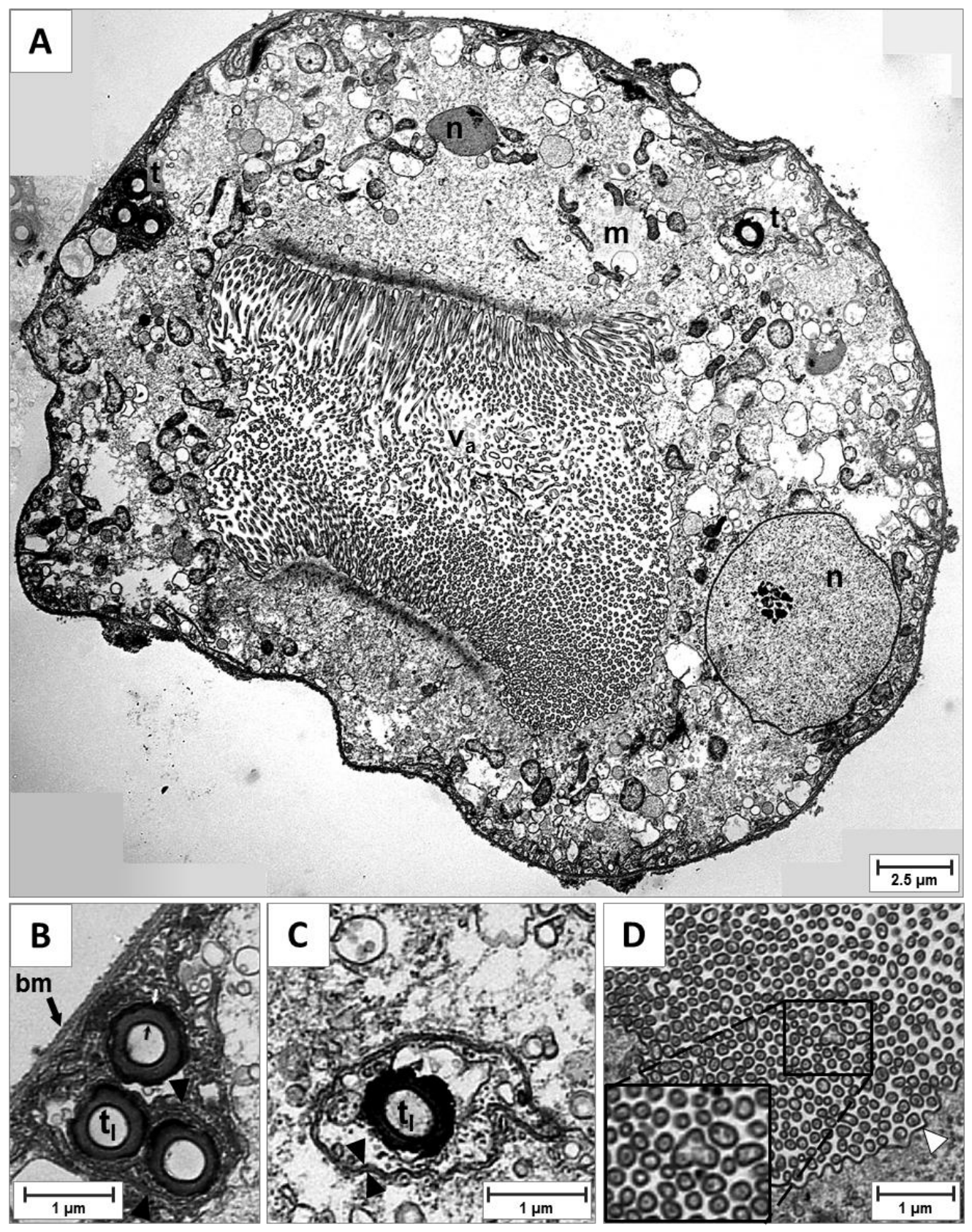

Figure 9: TEM of type 2 secretory gland cells. A Overview. The section contains at least two cells, as two nuclei ( $n$ ) can be observed. Note that differences in brightness and small cloudy zones are due to manual stitching of multiple single pictures. Gray squares on the outer edges have been added to cover close-by structures. B Close up of a bunch of three tubules $(\mathrm{t})$ and their carrier cells in cross-section. Tubule membranes (black and white small arrows) as well as the membranes of their carrier cells and the basal membrane (bm) that encloses the cell aggregate (thick black arrow), are indicated. C Close up of a single tubule and its carrying cell in cross-section. The lining cell membrane can be clearly seen. $\mathbf{D}$ Close up of vesicle $2 \mathrm{a}$ microvilli in cross-section. $\mathrm{m}$ : mitochondria, $\mathrm{t}_{\mathrm{l}}$ : tubule lumen, $\mathrm{v}_{\mathrm{a}}$ : vesicle of $2 \mathrm{a}$ cell, $\square$ vesicle membrane, cell membrane. 
The rather short distance between the vesicle lining membrane and the vesicular organelle is filled with strong filaments that dock to the organelle (Figure $8 A+C$ ), assuming that they give the organelle stability and help to keep it in place. In contrast, in vesicle 2a direct contacts between the microvilli and the organelle's head could not be observed. Instead, a fine mesh of thin microvilli seems to stabilize the organelle within the huge vesicle lumen (Figure $8 A+B$ ). Moreover, the head of the vesicular organelle seems to be porous at certain areas (not shown).

\subsection{Screens for the identification of genes involved in stink gland biology}

For the identification of candidate genes that play a role in stink gland development and physiology, three different approaches were at hand: (I) the iBeetle screen, a reverse genetics approach based on systemic RNA interference, (II) a forward genetics Gal4 enhancer trap screen, and (III) gland transcriptomics.

\subsection{1 iBeetle screen}

The iBeetle screen is an unbiased large-scale RNAi screen in the model organism $T$. castaneum (Schmitt-Engel et al., 2015). It was subdivided in a pupal injection part and a larval injection part. While the pupal screen identified late metamorphosis phenotypes and defects during embryogenesis, the larval screen focused on early metamorphosis and general morphological defects in the adults, for example altered ovaries and stink glands. All phenotypic features were recorded and collected in the iBeetle-Base (Dönitz et al., 2015).

\subsubsection{Identification of gland affecting genes in the iBeetle database}

For this study, the iBeetle database was searched for stink gland affecting genes after the first screening phase, when about 4480 genes had been analyzed in the larval screen, which resulted in 59 hits. Every hit is listed together with its corresponding official gene set number (OGS; Figure 10A). In addition, every iBeetle hit was maintained with an internal number (SG). The annotated phenotypes were grouped into seven different categories: I. secretion color darker, II. empty glands and/or necrotic gland tissue, III. irregular separation of gland compounds within the reservoir, IV. less secretion, V. irregular reservoir size (decreased or increased), VI. melanized gland content, and VII. colorless secretion. 


\section{RESULTS}

A

\begin{tabular}{|c|c|c|}
\hline SG & \# iB & OGS \\
\hline \hline 1 & iB_00069 & Tc_000316 \\
\hline 2 & iB_00081 & Tc_000379 \\
\hline 3 & iB_00110 & Tc_000504 \\
\hline 4 & iB_00185 & Tc_000885 \\
\hline 5 & iB_00414 & Tc_002616 \\
\hline 6 & iB_00754 & Tc_004698 \\
\hline 7 & iB_01044 & Tc_006408 \\
\hline 8 & iB_01236 & Tc_007650 \\
\hline 9 & iB_01315 & Tc_008186 \\
\hline 10 & iB_01372 & Tc_008608 \\
\hline 11 & iB_01440 & Tc_008936 \\
\hline 12 & iB_01644 & Tc_010033 \\
\hline 13 & iB_01798 & Tc_011075 \\
\hline 14 & iB_01814 & Tc_011159 \\
\hline 15 & iB_01910 & Tc_011969 \\
\hline 16 & iB_02292 & Tc_014494 \\
\hline 17 & iB_02297 & Tc_014520 \\
\hline 18 & iB_02301 & Tc_014544 \\
\hline 19 & iB_02367 & Tc_014967 \\
\hline 20 & iB_02401 & Tc_015095 \\
\hline
\end{tabular}

\begin{tabular}{|c|c|c|}
\hline SG & \# iB & OGS \\
\hline \hline 21 & iB_02416 & Tc_015165 \\
\hline 22 & iB_02428 & Tc_015203 \\
\hline 23 & iB_02471 & Tc_015379 \\
\hline 24 & iB_02516 & Tc_015811 \\
\hline 25 & iB_02517 & Tc_015818 \\
\hline 26 & iB_02542 & Tc_015993 \\
\hline 27 & iB_02563 & Tc_016254 \\
\hline 28 & iB_02584 & Tc_030051 \\
\hline 29 & iB_02625 & Tc_011255 \\
\hline 30 & iB_02633 & Tc_011371 \\
\hline 31 & iB_02673 & Tc_000240 \\
\hline 32 & iB_02692 & Tc_003063 \\
\hline 33 & iB_03401 & Tc_002550 \\
\hline 34 & iB_03552 & Tc_003409 \\
\hline 35 & iB_03637 & Tc_003857 \\
\hline 36 & iB_03693 & Tc_004126 \\
\hline 37 & iB_03695 & Tc_004129 \\
\hline 38 & iB_03780 & Tc_004533 \\
\hline 39 & iB_03913 & Tc_005167 \\
\hline 40 & iB_04066 & Tc_006098 \\
\hline
\end{tabular}

\begin{tabular}{|c|c|c|}
\hline SG & \# iB & OGS \\
\hline \hline 41 & iB_04137 & Tc_006423 \\
\hline 42 & iB_04205 & Tc_006735 \\
\hline 43 & iB_04797 & Tc_010251 \\
\hline 44 & iB_04839 & Tc_010449 \\
\hline 45 & iB_04850 & Tc_010484 \\
\hline 46 & iB_05264 & Tc_012539 \\
\hline 47 & iB_05278 & Tc_012610 \\
\hline 48 & iB_05284 & Tc_012642 \\
\hline 49 & iB_05329 & Tc_012828 \\
\hline 50 & iB_05331 & Tc_012834 \\
\hline 51 & iB_05342 & Tc_012857 \\
\hline 52 & iB_05442 & Tc_013513 \\
\hline 53 & iB_05518 & Tc_013892 \\
\hline 54 & iB_05584 & Tc_014205 \\
\hline 55 & iB_05712 & Tc_014870 \\
\hline 56 & iB_05719 & Tc_014887 \\
\hline 57 & iB_02627 & Tc_011288 \\
\hline 58 & iB_02716 & Tc_002723 \\
\hline 59 & iB_02931 & Tc_011812 \\
\hline
\end{tabular}

B

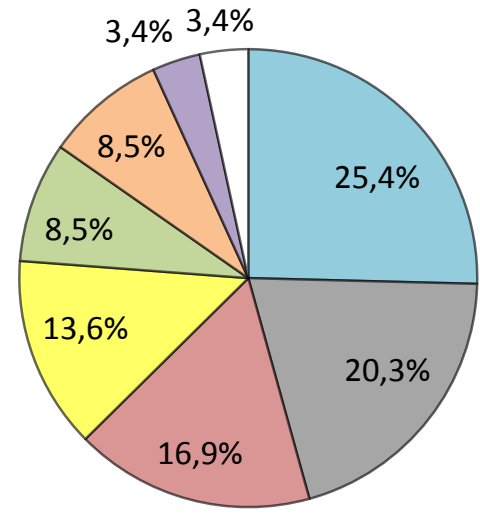

$\square \mathrm{I}$, secretion color darker

$\square$ II, empty/necrotic

$\square$ III, irregular separation of gland compounds

$\square \mathrm{IV}$, less secretion

$\square \mathrm{V}$, irregular reservoir size

$\square \mathrm{VI}$, melanized gland content

$\square$ VII, colorless secretion

$\square$ wrongly annotated

Figure 10: Stink gland phenotype categorization. A List of 59 iBeetle-Base hits annotated with a stink gland phenotype, accompanied by their corresponding official gene set numbers (OGS). SG stands for stink gland and represents an internal numbering for the ease of handling. The colored background fields depict the phenotype category to which a hit was assigned. See panel B for color code explanation. B Distribution of phenotypes to the different groups (I-VII) indicated by a color code. Note that two hits were wrongly annotated, therefore classified as 'wrongly annotated,' and excluded from further analysis. Percentages were rounded to one decimal place. 
RESULTS

The phenotypic classification for each hit is indicated by the color of the respective SG field in the list of Figure 10A. The distribution of phenotypes in the described categories is depicted in Figure 10B. Most gland phenotypes were annotated with 'darker secretion' (25.4\%). About onefifth showed 'empty/necrotic' glands, followed by about $17 \%$ displaying an 'irregular separation

of gland compounds', and $13.6 \%$ with 'less secretion'. With $8.5 \%$ each, only few hits were categorized as 'irregular reservoir size' and 'melanized gland content'. Least phenotypes (3.4\%) were ranked to the category 'colorless secretion'. Note that two hits were wrongly displayed as stink gland phenotypes at an early status of the iBeetle-Base. By now their annotations have been revised. As this mistake was not recognized until the rescreen, they were kept on the list among the 59 hits, but categorized as 'wrongly annotated'.

\subsubsection{Rescreen}

To confirm the stink gland phenotypes that were found in the iBeetle screen, 59 genes were rescreened by injecting dsRNA of fragments independent of the original iBeetle fragment into pupae of the San Bernardino strain. By using these so called non-overlapping fragments the risk of getting gland phenotypes due to off-target effects should be minimized. To be counted as a reproduced phenotype, two criteria had to be fulfilled: first, the phenotype of the rescreen had to be identical to the annotated one in the iBeetle screen, and secondly, this phenotype had to occur with a penetrance of at least $30 \%$, which was three out of ten injected animals per fragment.

In this way, the phenotypes of 32 genes (about 54\%) could be confirmed, but it should be noted that for some genes it was necessary to inject in larval stage and/or use higher dsRNA concentrations $(3 \mu \mathrm{g} / \mu \mathrm{l})$ to reproduce their phenotypes (data not shown). Exemplary pictures of altered glands after RNAi knockdown for each of the 32 confirmed genes are compiled in Figure 11. Some knockdown glands fit to more than one phenotype category. For example, there are good arguments for "SG10, III" to be put in the groups 'colorless secretion', 'melanized gland content', and 'irregular separation of gland compounds'. In such cases, individual decisions were made considering only the most striking feature. 


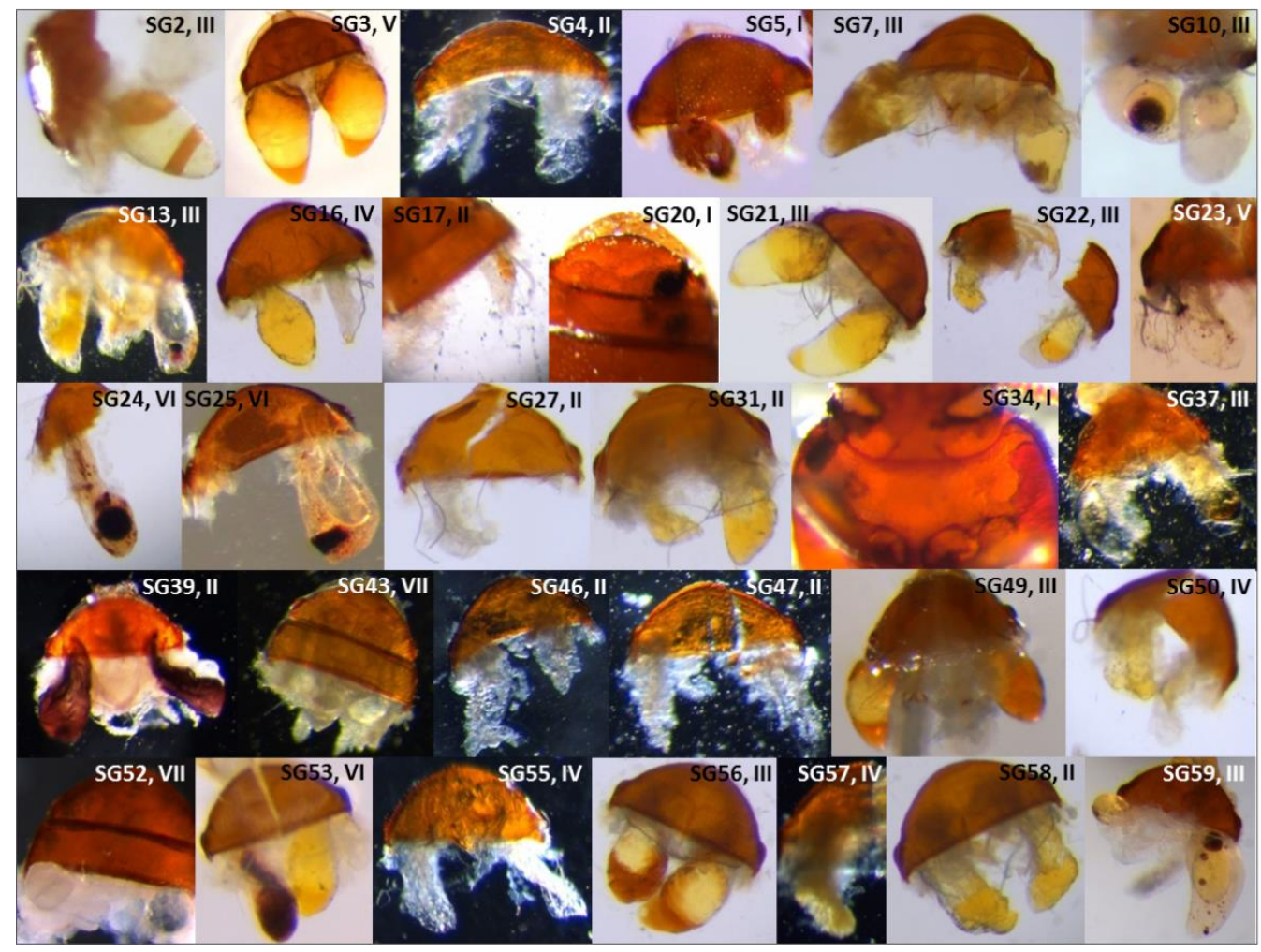

Figure 11: Morphological gland alterations after RNAi-mediated gene knockdown of 32 phenotypeconfirmed genes. Pictures are sorted by SG numbers and were taken either from own rescreen experiments or from the iBeetle-Base. Roman numbers indicate the phenotype category for each gene. I: 'secretion color darker'; II: 'empty/necrotic'; III: 'irregular separation of gland compounds'; IV: 'less secretion'; V: 'irregular reservoir size'; VI: 'melanized gland content'; VII: 'colorless secretion'.

In Figure 12 the distribution of confirmed and not confirmed genes within each phenotype category is displayed. All genes that counted for the categories 'irregular separation of gland compounds' and 'colorless secretion' could be confirmed in the rescreen, whereas phenotypes like 'secretion color darker', 'irregular reservoir size' and 'less secretion' were reproduced only in half or less of the primarily identified genes. Surprisingly, not all genes of the categories 'empty/necrotic' and 'melanized gland content' could be confirmed, although these phenotypes were clearly defined and easy to detect. Phenotypes that could not be reproduced had a wildtypic appearance in the rescreen. The chart in Figure 13 depicts the new distribution of phenotypes among the confirmed genes (compare Figure 10B). 
RESULTS

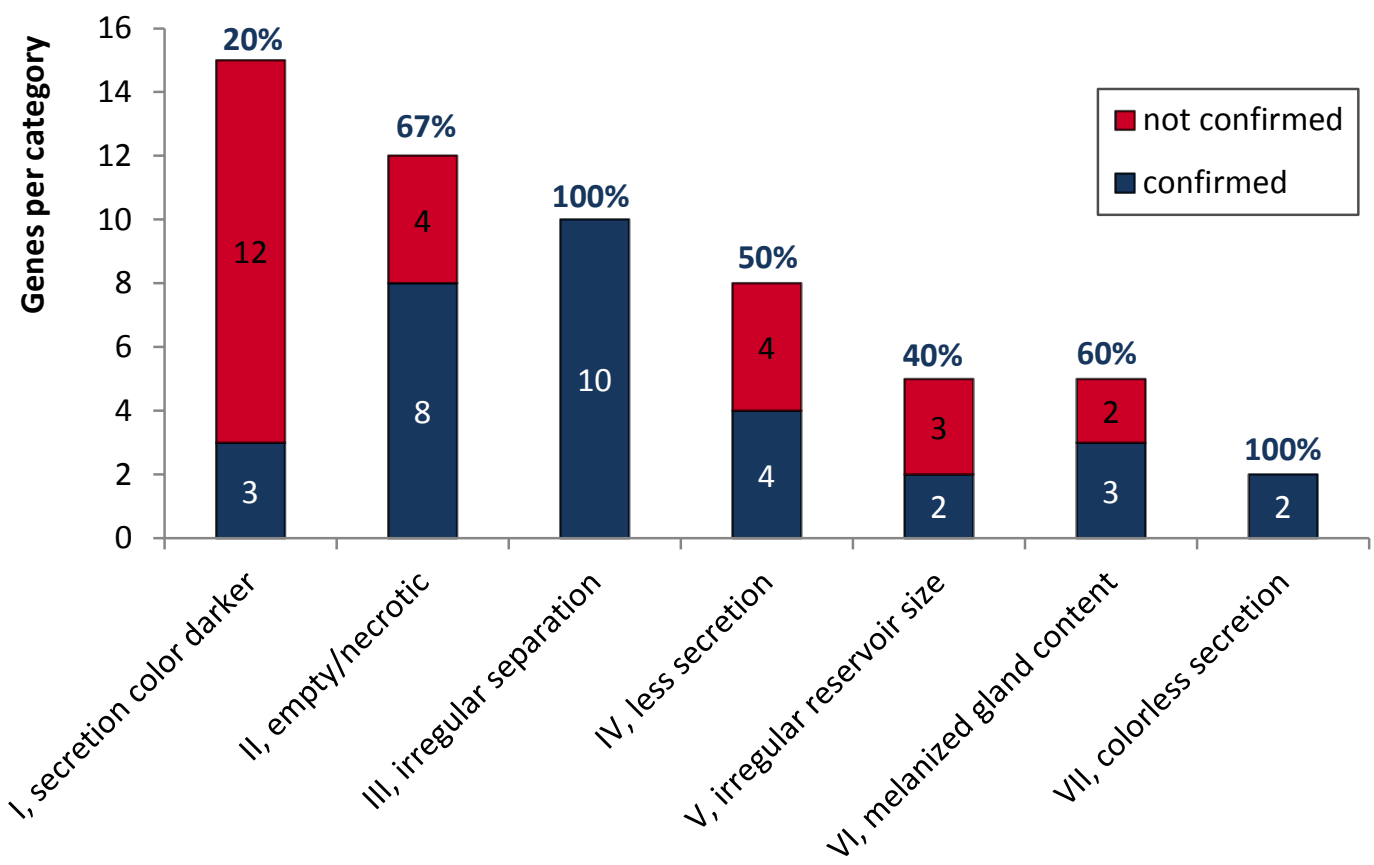

Figure 12: Rescreen statistics. Confirmed vs. not confirmed genes. Each bar represents one stink gland phenotype category. In blue filling and white font color the number of confirmed genes is depicted, whereas not confirmed phenotypes are indicated by red filling and black font. The blue percentage above each bar refers to confirmed phenotypes within the respective category. All indicated percentages were rounded to no decimal place.

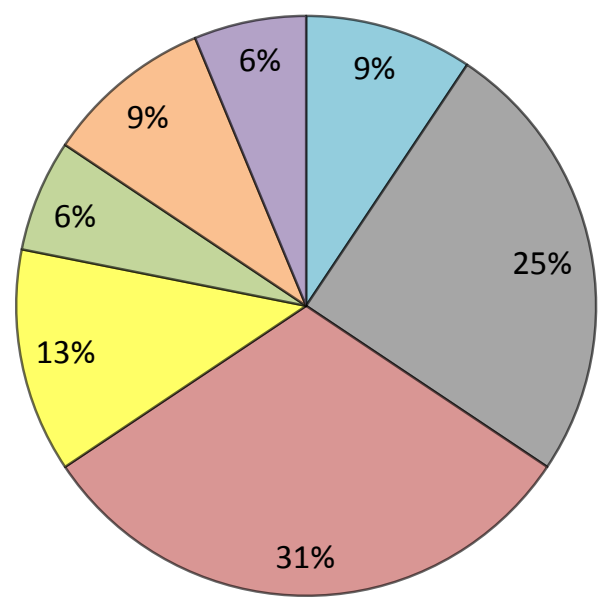

\author{
$\square \mathrm{I}$, secretion color darker \\ $\square$ II, empty/necrotic \\ $\square \mathrm{III}$, irregular separation of gland compounds \\ $\square \mathrm{IV}$, less secretion \\ $\square \mathrm{V}$, irregular reservoir size \\ $\square \mathrm{VI}$, melanized gland content \\ $\square$ VII, colorless secretion
}

Figure 13: Phenotype categories of the 32 confirmed genes. Updated distribution of phenotypes to the seven different groups I-VII. Indicated percentages were rounded to no decimal place. 


\section{RESULTS}

Gene ontologies of the 32 confirmed genes are summarized in Figure 14. Details are listed in Table 1. Tribolium sequences were taken from Genome Browser Greifswald (Tcas 3.0, 4.0, and 5.2) and analyzed for conserved domains as well as fly orthologues to deduce the rough molecular function. Based on their hypothesized molecular function, the 32 genes were divided into the following six groups: enzymes, transporters, channels, transcription factors, unknown function and others. Genes were ranked with unknown function, when neither conserved domains were detected nor any insect orthologue was found. The group 'others' mainly contained predicted receptors and activator molecules.

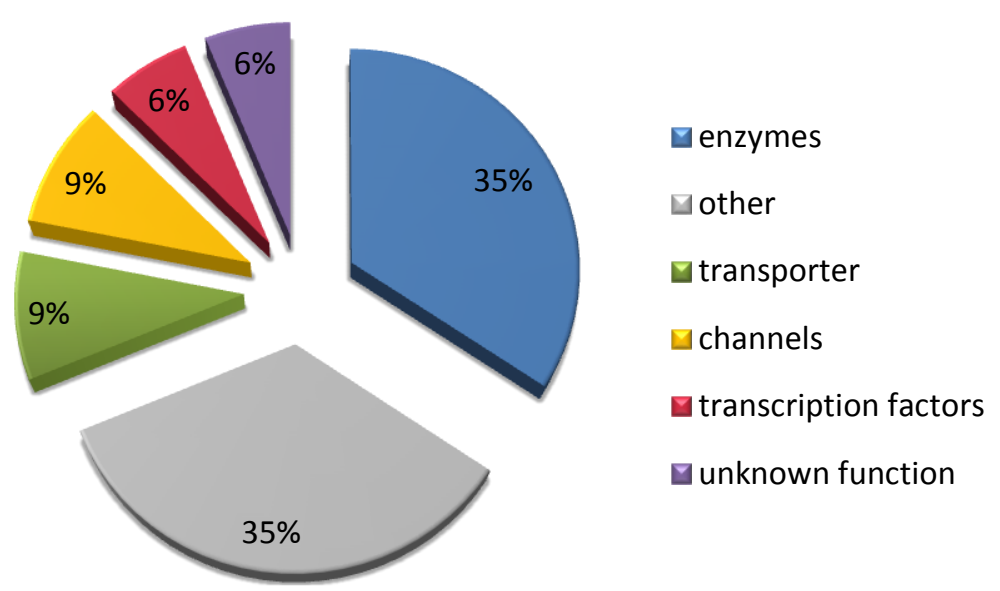

Figure 14: Gene ontology of the 32 confirmed genes. Genes were grouped by their predicted molecular function (see Table 1). All percentages were rounded to no decimal place. 
Table 1: Gene ontologies of the 32 confirmed stink gland phenotypes from the iBeetle screen. Conserved domains were identified by $\mathrm{NCBI}$ conserved domain search. Closest $D$. melanogaster fly homologs were taken from iBeetle-Base. The SG color code stands for the stink gland RNAi phenotype category of the respective gene and corresponds to the one displayed in Figure 13.

\begin{tabular}{|c|c|c|c|c|}
\hline SG & \# iB hit & OGS & conserved domains & fly ortholog/best hit \\
\hline 2 & iB_00081 & Tc_000379 & emp24/gp25L superfamily & eca - eclair (CG33104) \\
\hline 3 & iB_00110 & Tc_000504 & OTU-like cysteine protease superfamily & CG3251 - (CG3251) \\
\hline 4 & iB_00185 & Tc_000885 & none & CG14509 - (CG14509) \\
\hline 5 & iB_00414 & Tc_002616 & Aminoacid transporter superfamily & CG7888 - (CG7888) \\
\hline 7 & iB_01044 & Tc_006408 & Porin3 superfamily & porin - porin (CG6647) \\
\hline 10 & iB_01372 & Tc_008608 & Calcium-binding EGF-like domain; TSP_C superfamily & Tsp - Thrombospondin (CG11326) \\
\hline 13 & iB_01798 & Tc_011075 & Leucin rich repeats (LRR-8) & Sclp-Sclp (CG2471) \\
\hline 16 & iB_02292 & Tc_014494 & PTPA superfamily & $\begin{array}{l}\text { Ptpa - Phosphotyrosylphosphatase activator } \\
\text { (CG3289) }\end{array}$ \\
\hline 17 & iB_02297 & Tc_014520 & none & none \\
\hline 20 & iB_02401 & Tc_015095 & Major Facilitator Superfamily (MFS) & CG4797 - (CG4797) \\
\hline 21 & iB_02416 & Tc_015165 & V-SNARE_Csuperfamily & Gos28 - Golgi SNARE (CG7700) \\
\hline 22 & iB_02428 & Tc_015203 & NADB_Rossmann superfamily; FAR_Csuperfamily & wat - waterproof (CG1443) \\
\hline 23 & iB_02471 & Tc_015379 & NADB_Rossmann superfamily; FAR_C superfamily & CG1441 - (CG1441) \\
\hline 24 & iB_02516 & Tc_015811 & HP (Histidine Phosphatase) superfamily & Acph-1 - Acid phosphatase 1 (CG7899) \\
\hline 25 & iB_02517 & Tc_015818 & $\begin{array}{l}\text { Heavy-metal associated (HMA) domains --> copA (copper } \\
\text { exporting ATPase) }\end{array}$ & ATP7 - ATP7 (CG1886) \\
\hline 27 & iB_02563 & Tc_016254 & Gluzincin Peptidase superfamily; DUF3358 & CG8773 - (CG8773) \\
\hline 31 & iB_02673 & Tc_000240 & $\begin{array}{l}\text { Neurotransmitter-gated ion channel ligand binding and } \\
\text { transmembrane domain }\end{array}$ & GluClalpha - GluClalpha (CG7535) \\
\hline 34 & iB_03552 & Tc_003409 & NR-DBD-like superfamily & none \\
\hline 37 & iB_03695 & Tc_004129 & CUT superfamily; Homeodomain & onecut - onecut (CG1922) \\
\hline 39 & iB_03913 & Tc_005167 & Sec7 superfamily; Pleckstrin homology-like superfamily & siz - schizo (CG32434) \\
\hline 43 & iB_04797 & Tc_010251 & p450 superfamily & Cyp6a2 - Cytochrome P450-6a2 (CG9438) \\
\hline 46 & iB_05264 & Tc_012539 & $\begin{array}{l}\text { Immunglobulinesuperfamily; Down syndrome cell } \\
\text { adhesion moleculeC superfamily }\end{array}$ & $\begin{array}{l}\text { Dscam1 - Down syndrome cell adhesion } \\
\text { molecule } 1 \text { (CG17800) }\end{array}$ \\
\hline 47 & iB_05278 & Tc_012610 & ZnMc superfamily; disintegrin & mmd - mind-meld (CG42252) \\
\hline 49 & iB_05329 & Tc_012828 & none & Cpr92F - Cuticular protein 92F (CG5494) \\
\hline 50 & iB_05331 & Tc_012834 & CUB superfamily; Kelch superfamily; EGF-L superfamily & dsd - distracted (CG5634) \\
\hline 52 & iB_05442 & Tc_013513 & T-Box superfamily & mid - midline (CG6634) \\
\hline 53 & iB_05518 & Tc_013892 & JHBP-Superfamily & CG2016 - (CG2016) \\
\hline 55 & iB_05712 & Tc_014870 & $\begin{array}{l}\text { Fibronectin type } 3 \text { superfamily; Srchomology } 3 \text { domain } \\
\text { superfamily }\end{array}$ & Rbp-RIM-binding protein (CG43073) \\
\hline 56 & iB_05719 & Tc_014887 & NADB_Rossmann superfamily & antdh - antdh (CG1386) \\
\hline 57 & iB_02627 & Tc_011288 & ion channel superfamily & slo- slowpoke (CG10693) \\
\hline 58 & iB_02716 & Tc_002723 & Calponin homology domain; Filamin/ABP280 repeat & jbug - jitterbug (CG30092) \\
\hline 59 & iB_02931 & Tc_011812 & serine/threonine protein kinasesuperfamily & $\begin{array}{l}\text { Drak - Death-associated protein kinase } \\
\text { related (CG32666) }\end{array}$ \\
\hline
\end{tabular}




\section{RESULTS}

\subsubsection{Candidate genes from a Gal4-based enhancer trap screen}

A second approach for the identification of genes with roles in stink gland biology was a Gal4based enhancer trap screen. Eleven enhancer trap lines expressing tGFP in the glands have been found so far. For eight of them, the new integration sites could be determined, whereby one line (41067) had two integrations on separate chromosomes ( 3 and 7, respectively). Interestingly, lines 41123 and 41665, although obtained independently, had the insertion exactly on the same locus on chromosome 3 as line 41067, indicating a hotspot for the integration of the piggyBac transposon and suggesting that in line 41067 this integration site rather than the one on chromosome 7 is responsible for tGFP expression in the glands. The localization of the insertion for the three remaining lines (40093, 41142 and 41784) was not successful until now. Pictures of the lines with determined genomic integration loci are listed in Table 2. Note that pupae of line 41665 are representative for the tGFP expression pattern of pupae from lines 41067 and 41123 as well. In pupae of the enhancer trap lines 40011 and 41025, tGFP was only expressed in abdominal glands, whereas pupae of the other four lines showed fluorescing abdominal and prothoracic glands. Genomic integration in the eight lines occurred either directly into an exon or into non-coding regions between two genes (Figure 15). In four lines, the transposon jumped into regions between two genes. These neighbored genes were considered as possible enhancer targets and thus were knocked down to check for any alterations in the stink glands (Zhiyuan Shi, Bachelor thesis, Göttingen, 2014). However, no abnormalities were detected after pupal or larval injections, except for a high lethality of Tc_009984 knockdown at adult stage after pupal injection and Tc_012990 at larval stage. Much likely, the affected enhancer elements in the respective lines control genes at farther distance.

In the remaining two lines, the mutator element jumped into a coding region, affecting gene TC_007045 (line 41072) and Tc_013894 (line 40011), respectively. RNAi-mediated knockdown of these genes led to altered gland secretions (Figure 16). For Tc_007045, both gland types showed colorless secretions, whereas Tc_013894 RNAi resulted in colorless secretion in prothoracic glands and reduced light yellow abdominal gland content. It should be noted that the described gland phenotype of Tc_007045 was only observed in females. Due to their gland phenotypes both genes were chosen for further analysis (section 4.3). 
Table 2: Stink gland enhancer trap lines. Pictures show pupae of the indicated enhancer trap line from ventral (left) and dorsal site (right). Pupae of enhancer trap lines 40011 and 41025 display fluorescing abdominal glands only, whereas pupae of the other lines show tGFP expression in both abdominal and prothoracic glands. The genomic integration site was localized for every line via inverse PCR (Figure 15). In lines 40011 and 41072 the transposon inserted into an exon of Tc_013894 and Tc_007045, respectively. In all other lines the insertion site lies in between two genes. In these cases the directly neighbored genes upstream and downstream were considered as possible enhancer targets. Note that line 41665 is representative for two further lines (41067 and 41123) that show the same integration site.

\begin{tabular}{|c|c|c|c|c|}
\hline \multirow{2}{*}{ line } & \multirow{2}{*}{$\begin{array}{c}\text { tGFP expression } \\
\text { pattern }\end{array}$} & \multicolumn{3}{|c|}{ affected genes identified via inverse PCR } \\
\hline & & \# OGS & conserved domains & fly ortholog \\
\hline 40011 & & Tc_013894 & $\begin{array}{l}\text { Secreted trypsin-like serine } \\
\text { protease family }\end{array}$ & Corin (CG2105) \\
\hline \multirow{2}{*}{41025} & & Tc_002392 & $\begin{array}{l}\text { SIT4 phosphatase-associated } \\
\text { protein family }\end{array}$ & CG10289 \\
\hline & & Tc_002393 & $\begin{array}{l}\text { periplasmic serine protease } \\
\text { family }\end{array}$ & ssp6 - short spindle 6 (CG6619) \\
\hline \multirow{2}{*}{41041} & & Tc_015355 & Alpha/beta hydrolase family & CG8245 \\
\hline & $\theta$ & Tc_015376 & TRX-family & Trx-2 - thioredoxin-2 (CG31884) \\
\hline 41072 & & Tc_007045 & $\begin{array}{l}\text { Putative zinc finger in } \mathrm{N}- \\
\text { recognin (UBR box) }\end{array}$ & CG15141 \\
\hline \multirow{2}{*}{41389} & & Tc_012990 & $\begin{array}{l}\text { multiple Zinc finger double } \\
\text { domains }\end{array}$ & $\begin{array}{c}\text { Kr-h1 - Kruppel-homolog } 1 \\
\text { (CG45074) }\end{array}$ \\
\hline & & Tc_014818 & no CD's detected & no ortholog found \\
\hline \multirow{2}{*}{41665} & & Tc_010352 & lysozyme_like domain & CG8492 \\
\hline & & Tc_010353 & CD36 family & $\begin{array}{c}\text { Snmp2 - Sensory neuron } \\
\text { membrane protein } 2 \text { (CG7422) }\end{array}$ \\
\hline
\end{tabular}


RESULTS

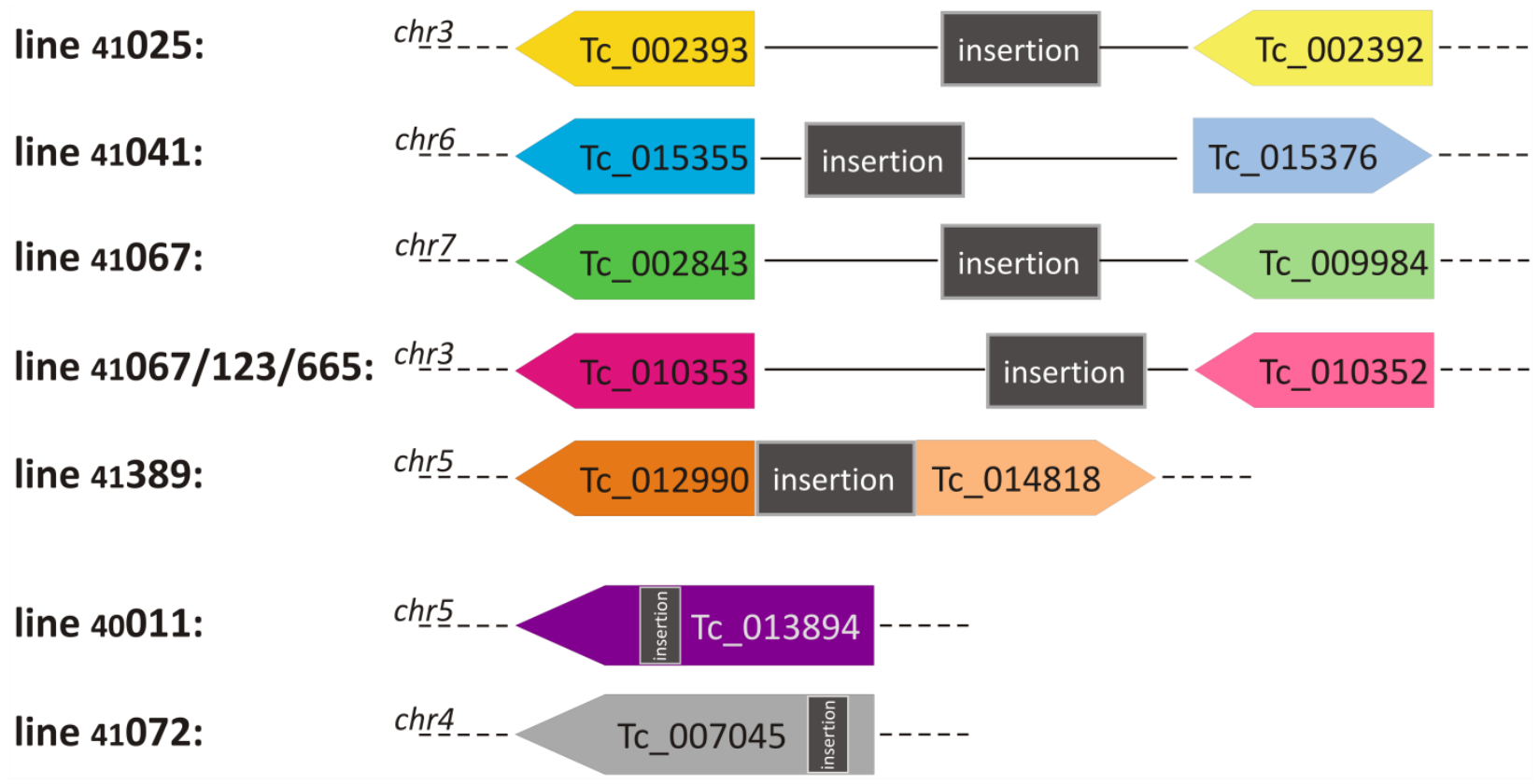

Figure 15: Transposon insertion sites of enhancer trap lines and relative distances to neighbored genes. Insertions occurred on chromosomes (chr) 3-7 and mainly in between two genes (colored arrows) as indicated for lines $41025,41041,41067,41123,41665$, and 41389 . Note that due to overlapping gene annotations for $T c_{-} 012990$ and $T c_{-} 014818$, the scheme depicts the insertion directly attached to both genes. For line 40011 and 41072 the transposon jumped into a coding region of the displayed gene. The insertion site between Tc_010353 and Tc_010352 (magenta) was obtained independently in three lines (41067, 41123, and 41665). For line 41067 an additional integration site was identified on chromosome 7 (green arrows).

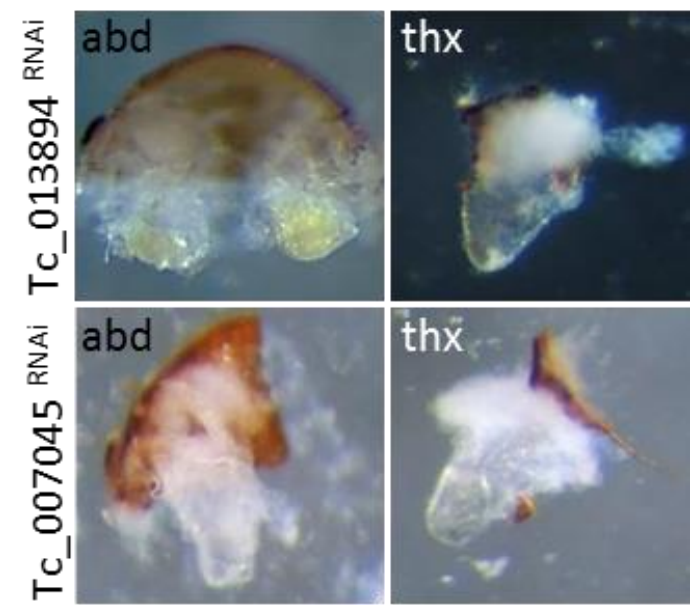

Figure 16: RNAi knockdown phenotypes of Tc_013894 and Tc_007045. Pictures were taken of dissected abdominal (abd, left) and prothoracic (thx, right) glands ten days after hatching of the injected pupae. Note that the phenotype depicted for Tc_007045 knockdown was only found in female beetles. 
Independent of known integration sites and knockdown gland phenotypes, all eleven lines are also of great interest for future transgenic experiments, for example a directed (over)expression of genes in different gland cell-types. Several attempts have been made by Zhiyuan Shi to figure out, which cell-type is marked by the reporter (tGFP) in every line. However, neither a direct dissection of pupal gland tissue, nor in situ hybridizations with a GAL4 RNA probe nor immunohistochemistry with anti-tGFP antibody on gland cryosections showed a clearly identifiable expression (data not shown).

\subsubsection{Gland transcriptome data}

A third approach for the identification of gland candidate genes was based on gland RNA-seq data, that had been produced and analyzed by Jianwei Li in his doctoral thesis (Li, 2013). He examined only the most 77 highly differentially expressed genes in the stink glands via RNAimediated knockdown. Subsequent gas chromatography-mass spectrometry analysis of RNAi gland volatile compounds identified genes that are essential for the beetle's biosynthesis of benzoquinones (Li et al., 2013). However, Dr. Li did not systematically analyze the function of glucosidases, phenoloxidases, and peroxidases, which are assumed to play critical roles in the production and chemical maintenance of benzoquinones as revealed in various enzymatic colorimetric assays by Georg Happ in 1968. In order to identify the genes that fulfill these predicted enzymatic functions in benzoquinone biosynthesis, in this study transcriptome data of Tribolium glucosidases, phenoloxidases, and peroxidases were analyzed in more detail for their differential expression in stink glands.

\subsubsection{Glucosidases}

Li et al. (2013) suggested four glucosidases (Tc_000223, Tc_000537, Tc_002741, and Tc_011354) to be possibly involved in precursor processing of benzoquinone production, based on their differential and high expression in stink gland tissue. Thus, in this study, these genes were functionally analyzed in RNAi-mediated knockdown experiments. However, none of those genes caused morphologically altered glands upon knockdown (data not shown). 


\section{RESULTS}

\subsubsection{Phenoloxidases}

Based on conserved domains and sequence similarity, 14 phenoloxidase (PO) genes, partly taken from Li et al. (2013), were identified in T. castaneum and grouped by their protein's substrate specificity and function into (I) hexamerins, (II) laccases, and (III) tyrosinases. Gene functions for most of the annotated Tribolium PO genes have been published previously and are listed in Table 3. Their differential expression in stink glands is depicted in Figure 17.

Table 3: Annotated phenoloxidases in T. castaneum. Lac2A and $B$ are splicing variants of $L a c 2$, whereby Lac2B $\left(^{*}\right)$ is not encoded by $T c_{-} 010490$ alone, but also shares the first five exons with splicing variant Lac2A (see text and Figure 18A+ B for details). 'ae6-8' stands for alternative exons 6-8. Note that the published Tribolium Lac2A sequence (AY884061) could be confirmed by RACE-PCR on stink gland cDNA of the $S B$ strain.

\begin{tabular}{|c|c|c|c|c|c|}
\hline \multirow{7}{*}{ 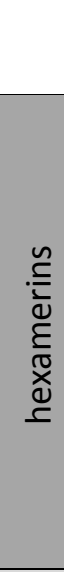 } & \# OGS & \multicolumn{2}{|c|}{ molecular function } & \multicolumn{2}{|c|}{$\begin{array}{c}\text { published under gene } \\
\text { name: }\end{array}$} \\
\hline & Tc_005374 & hexamerin 3 & \multirow{6}{*}{$\begin{array}{l}\text { no oxygen binding, } \\
\text { amino acid source } \\
\text { during metamorphosis } \\
\text { of holometabolous } \\
\text { insects }\end{array}$} & TcaHEX3 & \multirow{6}{*}{$\begin{array}{c}\text { Cristino et al., } \\
2010\end{array}$} \\
\hline & Tc_005375 & hexamerin 4 & & TcaHEX4 & \\
\hline & Tc_005376 & hexamerin $1 \mathrm{~A}$ & & TcaHEX1A & \\
\hline & Tc_005377 & hexamerin $1 \mathrm{~B}$ & & TcaHEX1B & \\
\hline & Tc_006515 & hexamerin 5 & & TcaHEX5 & \\
\hline & Tc_006769 & hexamerin 2 & & TcaHEX2 & \\
\hline \multirow{4}{*}{$\begin{array}{l}\mathscr{U} \\
\stackrel{⿹}{0} \\
\stackrel{U}{0} \\
\underline{0}\end{array}$} & Tc_000821 & laccase 1 & $\begin{array}{l}\text { not involved in } \\
\text { sclerotization }\end{array}$ & TcLac1 & \multirow{3}{*}{$\begin{array}{c}\text { Arakane et al. } \\
2005\end{array}$} \\
\hline & Tc_010489 & laccase $2 \mathrm{~A}$ & \multirow{2}{*}{$\begin{array}{l}\text { responsible for beetle } \\
\text { cuticle tanning }\end{array}$} & TcLac2A & \\
\hline & Tc_010490 & laccase 2 ae6-8 & & TcLac2B* & \\
\hline & Tc_015880 & $\begin{array}{c}\text { multicopper } \\
\text { oxidase- } \\
\text { related protein }\end{array}$ & no oxidase activity & TcMCORP & $\begin{array}{l}\text { Peng et al., } \\
2014\end{array}$ \\
\hline \multirow{4}{*}{ 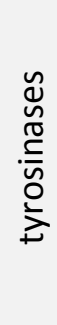 } & Tc_000325 & tyrosinase 1 & \multirow{2}{*}{$\begin{array}{l}\text { melanin formation, } \\
\text { wound healing, } \\
\text { insect immune defense }\end{array}$} & TcTyr1 & \multirow{2}{*}{$\begin{array}{c}\text { Arakane et al. } \\
2005\end{array}$} \\
\hline & Tc_014907 & tyrosinase 2 & & TcTyr2 & \\
\hline & Tc_015848 & tyrosinase $2-\mathrm{N}$ & \multicolumn{3}{|c|}{ not described } \\
\hline & Tc_015849 & tyrosinase $2-\mathrm{C}$ & \multicolumn{3}{|c|}{ not described } \\
\hline
\end{tabular}




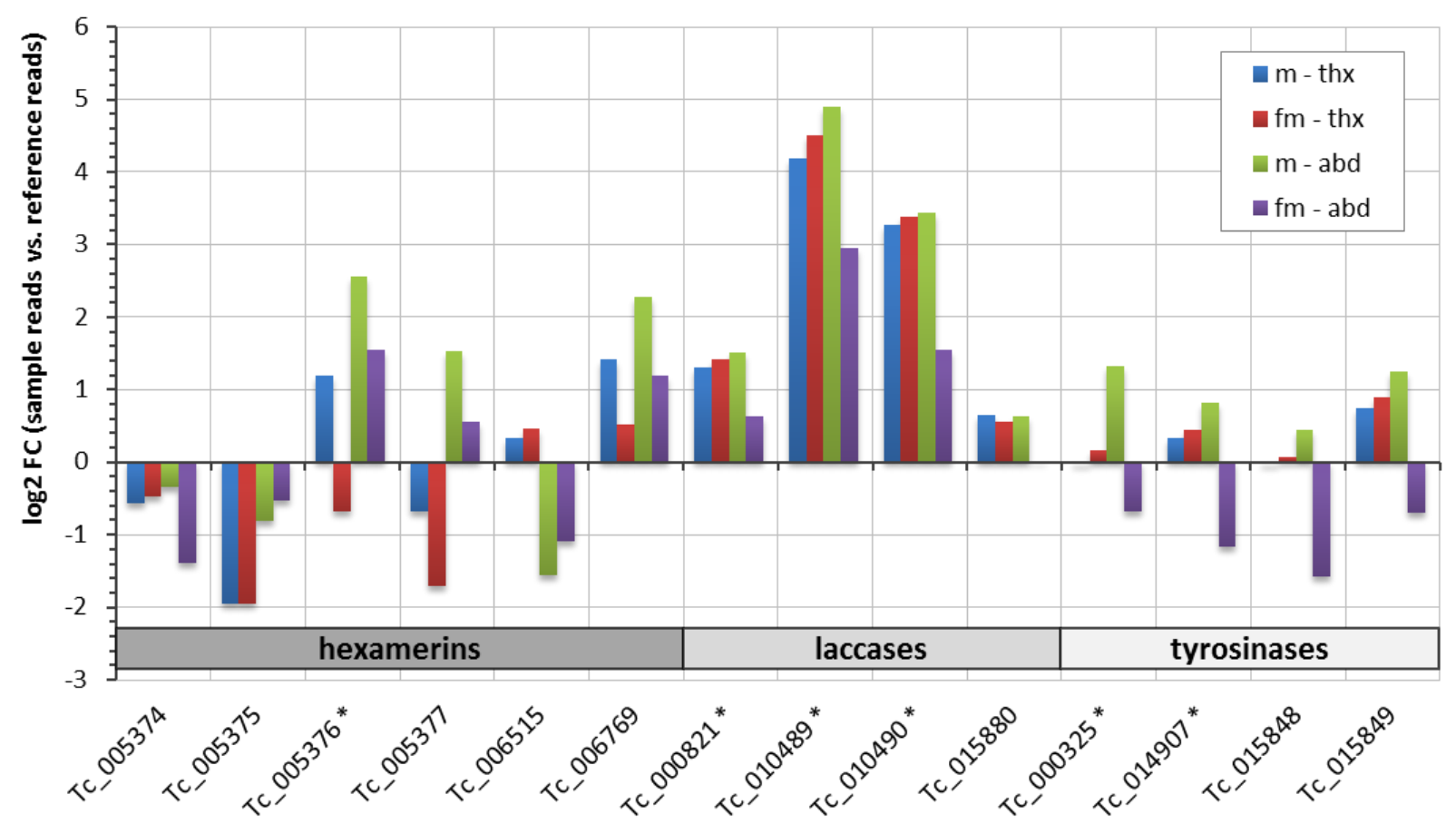

Figure 17: Differential expression of $T$. castaneum phenoloxidase genes in stink glands. 14 genes were identified and six were selected for further analysis (marked with asterisk). The corresponding dataset as well as actual read counts are displayed in the appendix (7.2: Tables 8+9). FC: fold change, m: male beetle, fm: female beetle, abd: abdominal gland, thx: prothoracic gland.

To identify candidates that possess phenoloxidase activity relevant in the glands, genes were selected for RNAi knockdown experiments, based on their expression level and known molecular function. As it is known that hexamerins have lost their oxidizing function (Cristino et al., 2010), only HEX1a (Tc_005376), which shows the highest differential expression in stink gland tissue, was included. In the laccase group, Lac1 (TC_000821) and Lac2 with both splicing variants were chosen. While $L a c 2 A$ corresponds to $T C_{-} 010489$, Lac2B consists of shared first five exons from Tc_010489 but an alternative exon set 6-8, which is annotated as Tc_010490 (Figure 18A). TCMCORP (Tc_015880) was excluded as no oxidase activity could be detected for the laccase-like protein in a previous study (Peng et al., 2014). Both tyrosinases Tyr1 (Tc_000325) and Tyr2 (Tc_014907) were selected, whereas additionally annotated fragments of Tyr2 (TC_015848 and TC_015849, corresponding to the $\mathrm{N}$-terminal and C-terminal part of tyrosinases 2 protein) were not considered for further experiments. In sum, HEX1A, Lac1, Lac2, Tyr1 and Tyr2 were knocked down via RNAi. 
A

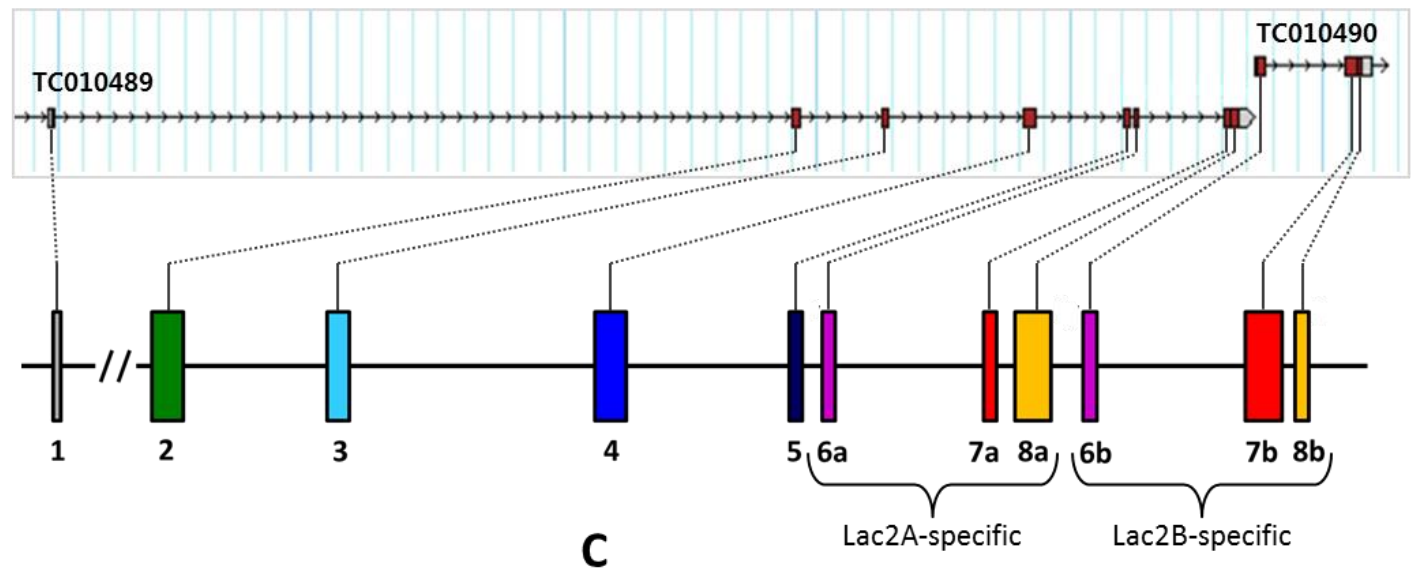

B
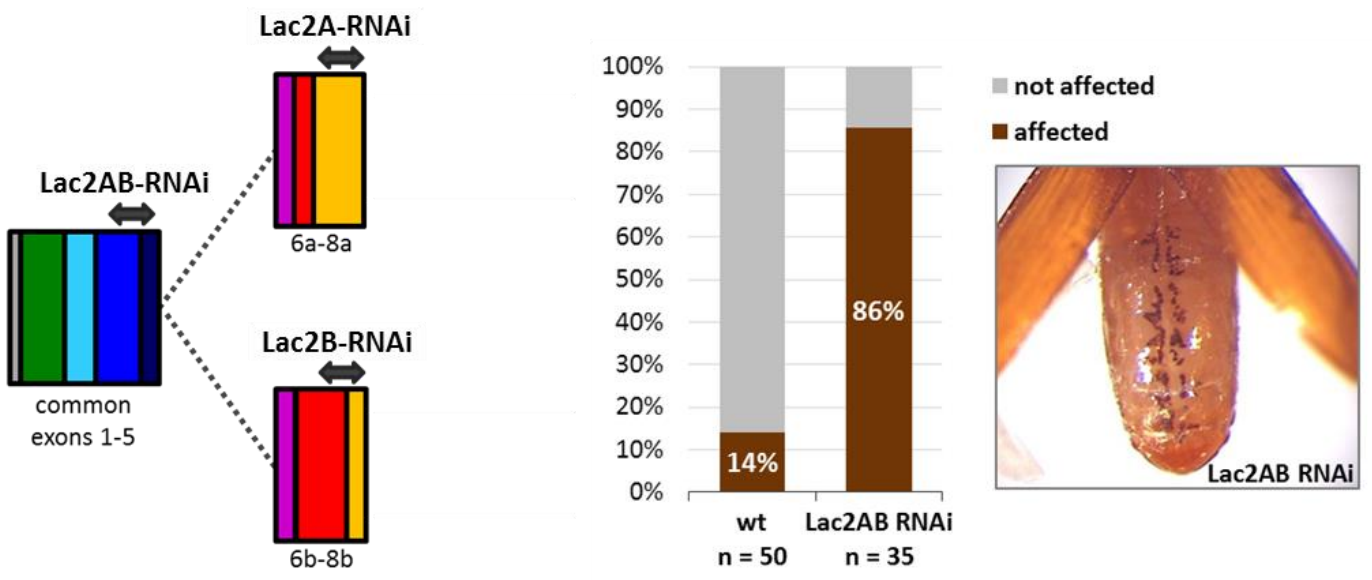

D

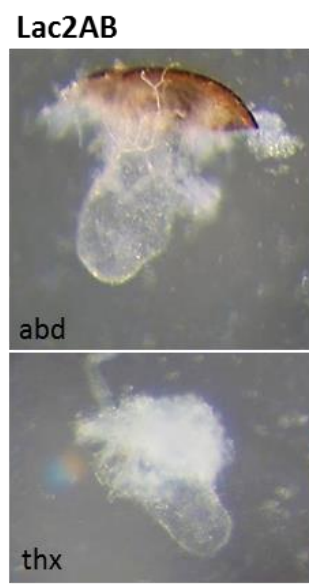

Lac2A

Lac2B

Tyr1

wt
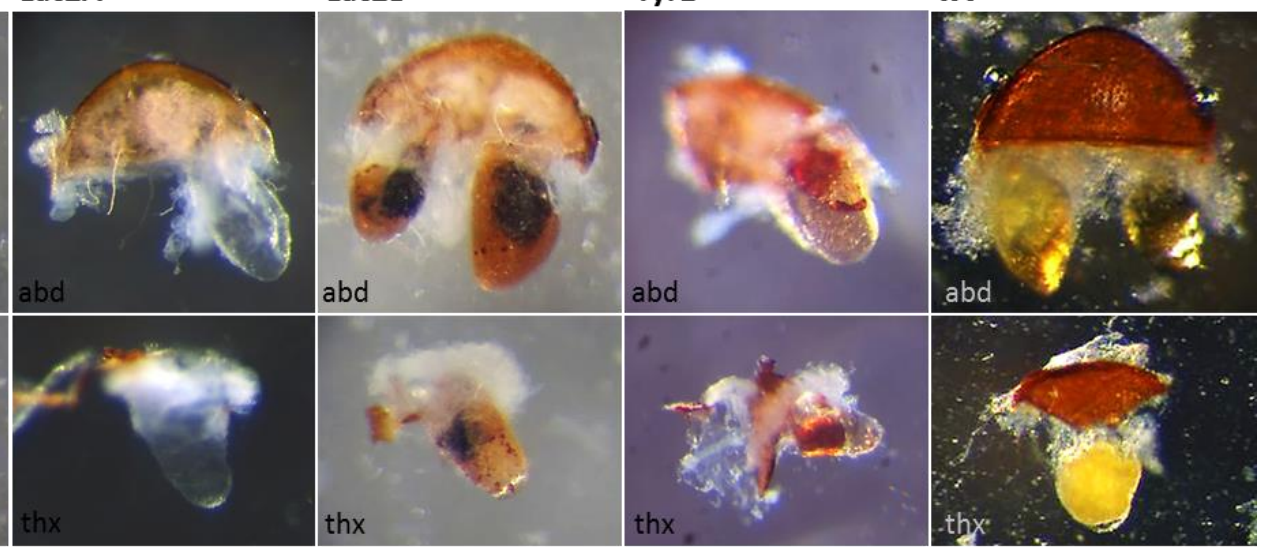

Figure 18: RNAi mediated knockdown of selected phenoloxidase genes. A Gene structure of Tribolium Lac2. Exons are displayed as colored bars and are linked to their corresponding exons from the gene annotation on GBrowse 5.2. Alternative exon sets are marked with A and B (after Dittmer and Kanost, 2010). B Selected Lac2 RNAi fragments (gray double arrows). For knockdown of both splicing variants dsRNA was produced from a sequence spanning the border of common exons 4 and 5 (Lac2AB). RNAi fragments for $\operatorname{La} 2 A$ and $L a c 2 B$ were constructed in regions with highest sequence difference (exon $8 a$ for $\operatorname{Lac} 2 A$ and exon border $7 / 8$ for $L a c 2 B$, respectively). C Number of beetles showing melanotic dots 
along the central nerve system in the abdomen upon $\angle a c 2 A B$ double knockdown compared to wildtype (wt). D Gland phenotypes upon Lac2 and Tyr1 knockdown. Phenotypes are displayed for abdominal (abd) and prothoracic (thx) glands. For Lac2, both splicing variants were knocked down separately (Lac2A and $L a c 2 B$, respectively) and one double knockdown was performed using dsRNA that targeted a sequence in the shared part of both variants $(\operatorname{Lac} 2 A B)$.

For Lac2 knockdown, three different dsRNAs were injected, one only directed against splicing variant $A$ (exon $8, \operatorname{Lac} 2 A$ ), a second only for the knockdown of splicing variant $B$ (spanning exon border $7 / 8, L a c 2 B$ ) and a third that targeted both variants spanning exon border 4/5 (Lac2AB; Figure 18B). Whereas glands of HEX1A, Lac1, and Tyr2 knockdown beetles were not detectibly different from wildtype (not shown), strong aberrant gland phenotypes could be detected in Lac2 and Tyr1 knockdowns (Figure 18D). The knockdown of each of the transcripts (Lac2A, $L a c 2 B, L a c 2 A B, T y r 1)$ caused severely altered gland contents. Lac2A deficient glands displayed colorless secretions, whereas the lack of splicing variant B led to darker and partly melanized gland content. Interestingly, the double knockdown had the same appearance as the single knockdown of Lac2A. For Tyr1 knockdown the main represented phenotype had areas with red secretion separated from the yellow content. In rare cases the secretions were light yellow with numerous little red dots equally distributed inside (not shown). Both genes, Lac2 and Tyr1, were selected for further analysis of gland volatile composition in their knockdowns via GC-MS (section 4.3). In addition to altered glands upon Lac2 knockdown, it was also observed that the number of beetles showing melanotic-like dots along their anterior-posterior axis next to the central nervous system in the abdomen was drastically increased in the knockdowns. Whereas only $14 \%$ of wildtype beetles possessed the dark-brown capsules, they could be detected in $86 \%$ of the Lac2AB deficient beetles (Figure 18C). Also, the number of the capsules seemed to be much higher in $L a c 2 A B$ knockdown beetles than in wildtype beetles but have not been analyzed statistically. Moreover, the beetle cuticle tanning process was delayed and beetles died 20 days after hatching at latest, many of them already within the first ten days (not shown), which is similar to published data from Arakane et al. (2005). 


\section{RESULTS}

\subsubsection{Peroxidases}

28 peroxidase genes were identified in $T$. castaneum and grouped into seven peroxidase families based on conserved domains. In addition, every predicted gene was analyzed for a signal peptide in its encoded protein (Figure 19A), as the model for benzoquinone biosynthesis hypothesized an enzyme with peroxidase activity in the tubule carrying cell (Happ, 1968; introduction 2.4.3), which might be secreted into the lumen of the tubule and therefore contained in the secretion.

\begin{tabular}{|c|c|c|}
\hline \# OGS & predicted molecular function & secreted protein \\
\hline Tc_010354 & \multirow{3}{*}{ glutathione-peroxidases } & no \\
\hline Tc_010355* & & no \\
\hline Tc_010362* & & yes \\
\hline Tc_001071* & \multirow{8}{*}{ peroxiredoxins } & yes \\
\hline Tc_001700* & & no \\
\hline Tc_004948* & & no \\
\hline Tc_007526 & & no \\
\hline Tc_007642* & & no \\
\hline Tc_012328* & & no \\
\hline Tc_013791* & & no \\
\hline Tc_014929* & & no \\
\hline Tc_000175* & \multirow{8}{*}{$\begin{array}{l}\text { peroxinectin-like animal } \\
\text { heme peroxidase }\end{array}$} & yes \\
\hline Tc_000751 & & yes \\
\hline Tc_004551 & & no \\
\hline Tc_004579 & & no \\
\hline Tc_004661 & & yes \\
\hline Tc_005493* & & yes \\
\hline Tc_011222 & & yes \\
\hline Tc_015234 & & yes \\
\hline Tc_001556 & peroxidasin & yes \\
\hline Tc_002498 & \multirow{2}{*}{$\begin{array}{l}\text { (Duox) Dual oxidase and rel. } \\
\text { animal heme peroxidases }\end{array}$} & yes \\
\hline Tc_004592 & & yes \\
\hline Tc_004593 & \multirow{3}{*}{$\begin{array}{c}\text { NOX_Duox_like_FAD_NADP } \\
\text { (NADPH-Oxidase) }\end{array}$} & no \\
\hline Tc_013282 & & no \\
\hline Tc_013283 & & no \\
\hline Tc_000083 & \multirow{3}{*}{ catalase } & no \\
\hline Tc_011090 & & no \\
\hline Tc_011385 & & no \\
\hline
\end{tabular}

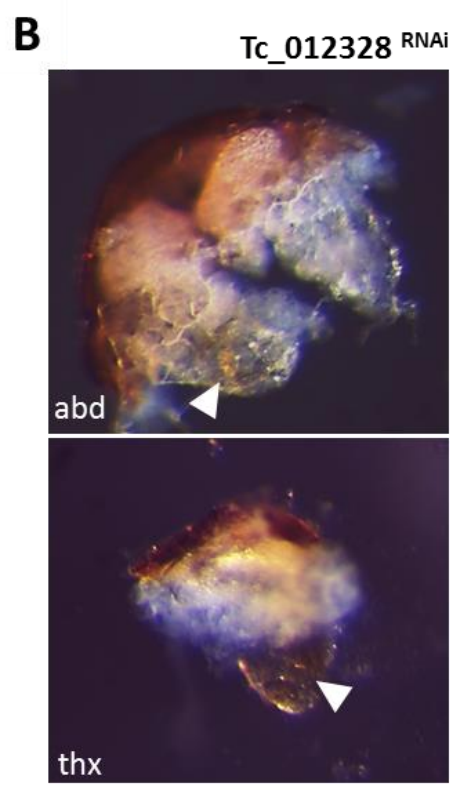

Figure 19: T. castaneum peroxidases. A 28 annotated peroxidases were grouped into the seven displayed peroxidase families. Signal peptide analysis was performed to identify secreted peroxidases. Asterisks mark the peroxidases selected for further analysis. Peroxiredoxin Tc_012328 is highlighted. B Gland phenotype after RNAi knockdown of Tc_012328. White arrows point to light yellow secretion in the reservoir of abdominal (abd) and prothoracic (thx) glands. 
The differential expression levels of these peroxidases are depicted in Figure 20. Only few of them showed enriched expression in stink glands. Transcripts of most peroxidase genes were strongly underrepresented in the glands compared to the reference tissue. For RNAi knockdown experiments, two glutathione peroxidases (Tc_010355, Tc_010362), all peroxiredoxins except for the not expressed one (TC_007526), and two peroxinectin-like peroxidases (TC_000175, Tc_005493) were selected. Selection was based mainly on high differential expression levels and the feature of being secretable. Catalases were not included as it was assumed that their cellular function of breaking down reactive oxygen species is much likely not linked to benzoquinone production and maintenance. For the peroxinectin-like peroxidases, one enriched and one underrepresented gene was chosen randomly from the secretable candidates. After RNAi-mediated knockdown of the selected genes, only one of the peroxiredoxins, Tc_012328, displayed altered gland content. The reservoirs contained less light yellow secretion in both types of glands (Figure 19B). However, this protein is much likely not secreted (Figure 19A).

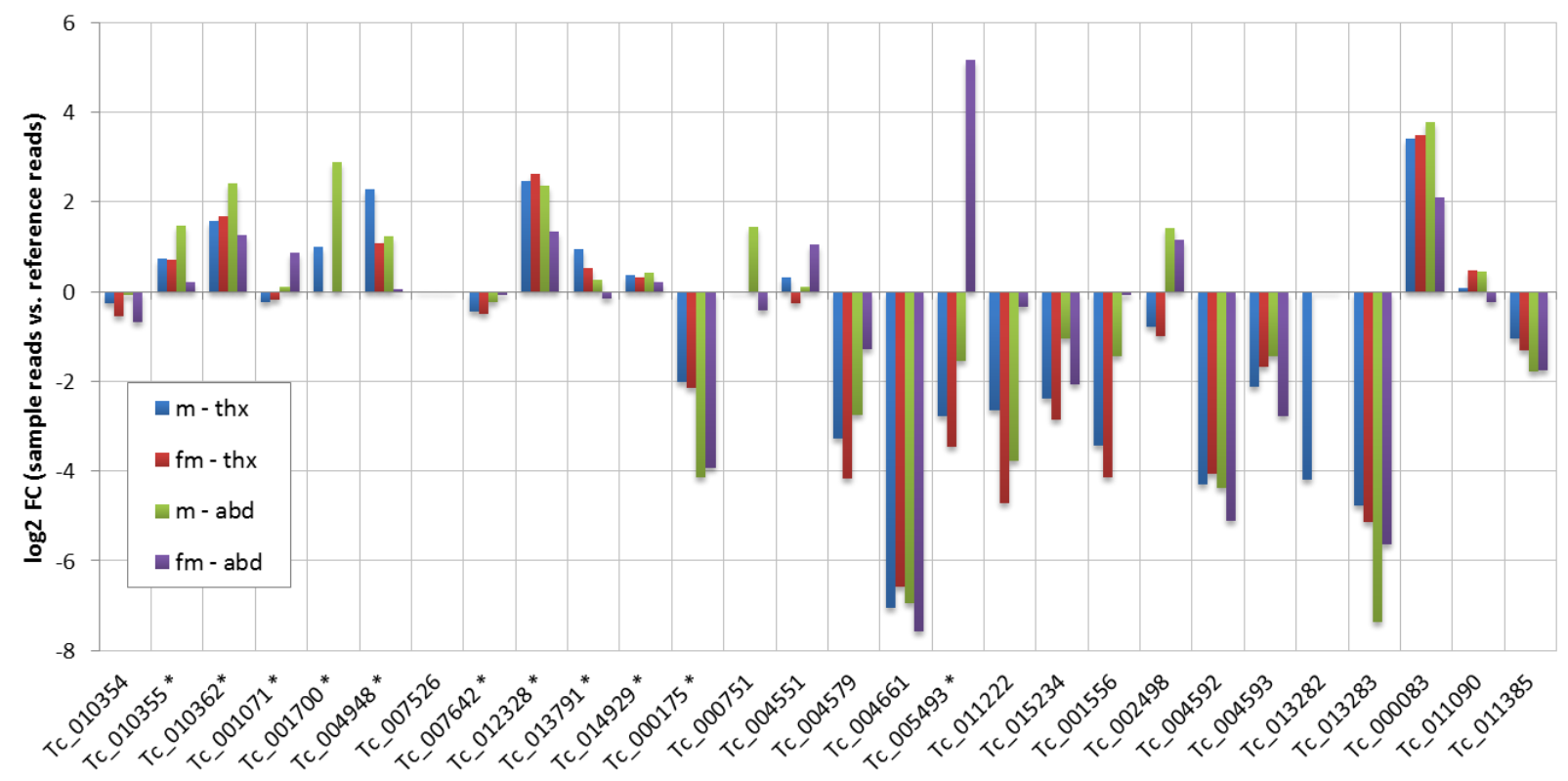

Figure 20: Differential expression of $T$. castaneum peroxidase encoding genes in stink glands. 28 genes were identified. Peroxidases selected for RNAi knockdown analysis are marked with an asterisk. Missing bars are either due to the fact that the respective gene is neither expressed in the glands nor in the reference tissue (beetle mid-abdomen) or to a minimal difference between gland sample and reference, which is badly resolved in the chart. The corresponding dataset as well as actual read counts are displayed in the appendix (7.2: Tables 10+11). FC: fold change, m: male beetle, fm: female beetle, abd: abdominal gland, thx: prothoracic gland. 


\section{RESULTS}

To deduce a possible molecular function of peroxiredoxin Tc_012328, its coding sequence was confirmed via RACE-PCR and translated into protein sequence (see appendix 7.7 for nucleotide and aa-sequence). Subsequently, a phylogenetic tree with insect (T. castaneum and $D$. melanogaster) and mammal ( $H$. sapiens and $M$. musculus) peroxiredoxins was calculated (Figure 21). Amino acid sequences clustered in five peroxiredoxin families with at least one Tribolium peroxiredoxin per family (alignment in appendix 7.5). Phylogenetic analysis suggested Tc_012328 as peroxiredoxin 3 ( $\operatorname{Prdx} 3)$, which could be confirmed by an expanded signal peptide analysis of all peroxiredoxins used in the phylogenetic tree (see appendix 7.3). In agreement with all other Prdx3, Tc_012328 was also predicted with a mitochondrial target peptide. Thus, Tc_012328 was designated as Tribolium peroxiredoxin 3 ( $\operatorname{Prdx} 3)$.

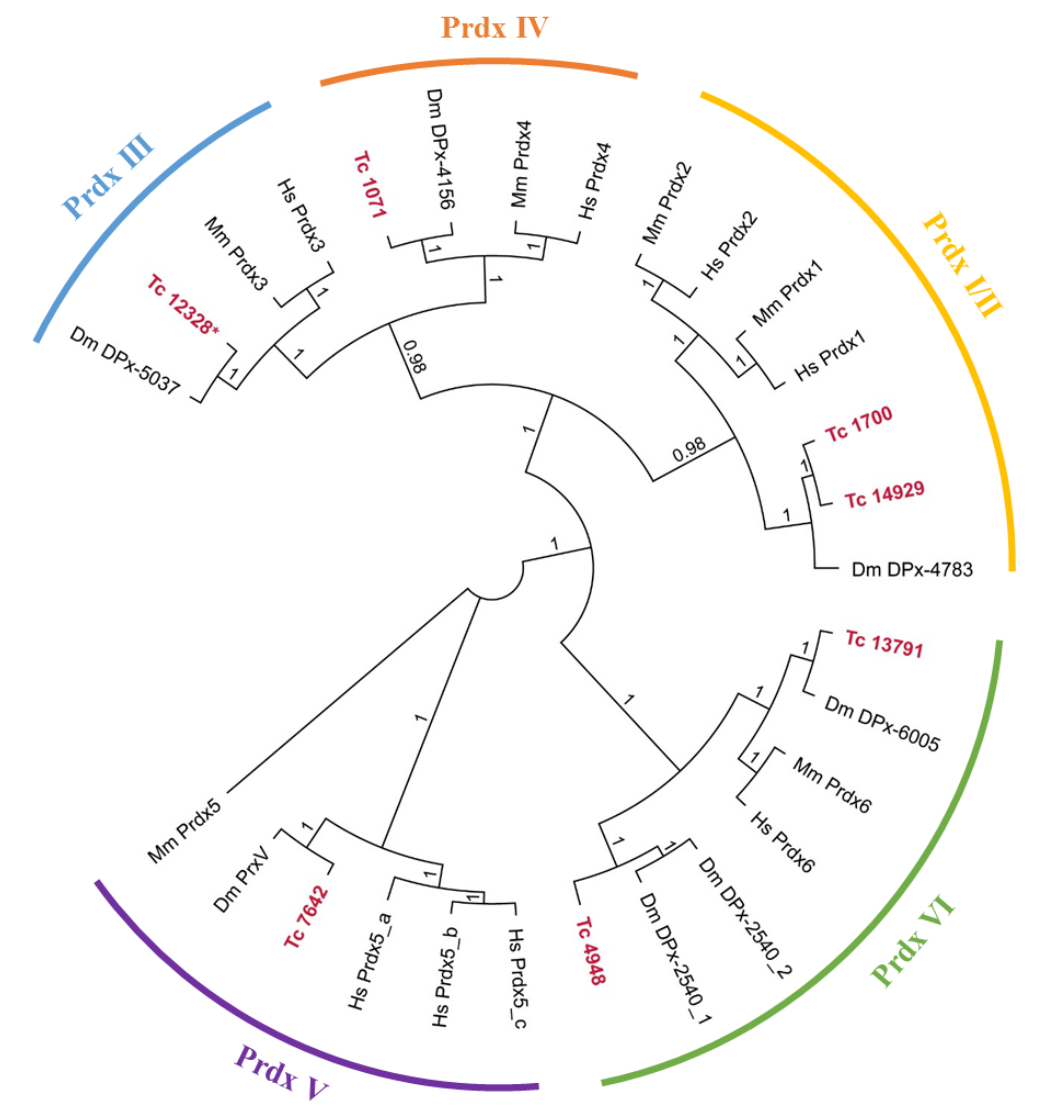

Figure 21: Phylogenetic tree of insect and mammal peroxiredoxins. Peroxiredoxins cluster in five subfamilies (Prdx I/II to PrdxVI) with at least one Tribolium peroxiredoxin (red) per family. Tc_012328 is marked additionally with an asterisk and clusters with members of the PrdxIII family. The tree was constructed with MrBayes, based on ClustalW alignment of full amino acid sequences. Mm Prdx5 was set as outgroup. Numbers at nodes indicate probabilities. Accession numbers are displayed in section 3.4.3. Dm: D. melanogaster, Hs: H. sapiens, Mm: M. musculus, Tc: T. castaneum. 
RESULTS

\subsection{Gas chromatography-mass spectrometry analysis of gland secretion volatiles}

The iBeetle screen, the enhancer trap screen, and the transcriptomic data analysis revealed a large number of candidates with a possible role in gland secretion production. To validate and specify their obtained RNAi knockdown phenotypes, GC-MS analysis was performed with gland content of ten days-old RNAi knockdown beetles in comparison to gland content of bufferinjected beetles at same age (referred to as wt in the following). For every knockdown, abdominal and prothoracic gland contents were measured separately. But as the dissection of intact prothoracic gland reservoirs was difficult, much more reliable data were achieved from abdominal gland measurements and will be presented here. Nevertheless, raw data of prothoracic gland measurements were kept and are displayed in the appendix (7.6). Note that the following results respect to the mean of two independent measurements, unless indicated otherwise.

For the 32 iBeetle genes, the gland contents of two individuals (one male and one female beetle) were mixed and measured in one sample. In Table 4, the GC-MS analysis is summarized for each gene. Abundances for each chemical was calculated as percentage of the respective wildtype abundance, whereby $100 \%$ indicated wildtype level of a chemical, $>100 \%$ an increase and $<100 \%$ a reduction of the respective chemical compared to wildtype levels. In total, 14 of the 32 genes, which have been detected with a morphological phenotype in the rescreen, also displayed a GC-MS phenotype in terms of secretion volatile abundance. Seven of them (red type) were observed with strongly reduced amounts of the benzoquinones (MBQ and $E B Q$ ) as well as the alkenes (C-15 and C-17). Slightly reduced levels of BQ's or BQ's and alkenes were obtained for four genes (gray fields). Most striking were three genes, Tc_010251, Tc_011812, and Tc_015818, that upon knockdown showed no benzoquinones but wildtypic levels for the alkenes (Figure 22C-E) and were henceforth called benzoquinone-less genes (BQ-less). Rescreen and GC-MS analysis data for Tc_015818, encoding a copper transporting ATPase (Atp7), is published as an example for gland phenotypes in the major iBeetle publication with coauthorship (Schmitt-Engel et al., 2015). 


\section{RESULTS}

Table 4: GC-MS analysis of 32 confirmed iBeetle genes. For each gene knockdown, the gland content of one male and one female was measured together (mixed genders), but with abdominal and prothoracic gland content measured separately. Here, only abdominal gland data are presented. Abundances for each substance is calculated in percent of the respective wildtype abundance $(100 \%=w t, 50 \%=$ half amount of wt and so on). Black fields mark benzoquinone-less genes (BQ-less), gray fields accentuate genes with milder GC-MS phenotype. Genes with both affected BQ- and alkene levels are highlighted in red. $M B Q:$ 2-methyl-1,4-benzoquinone, EBQ: 2-ethyl-1,4-benzoquinone , 1-C15: 1-pentadecene, 1-C17: 1-heptadecene.

\begin{tabular}{|c|c|c|c|c|c|}
\hline \multirow{2}{*}{ screen } & \multirow{2}{*}{ gene } & \multicolumn{4}{|c|}{ mixed genders } \\
\hline & & $M B Q$ & $E B Q$ & $1-C 15$ & $1-C 17$ \\
\hline \multirow{32}{*}{ 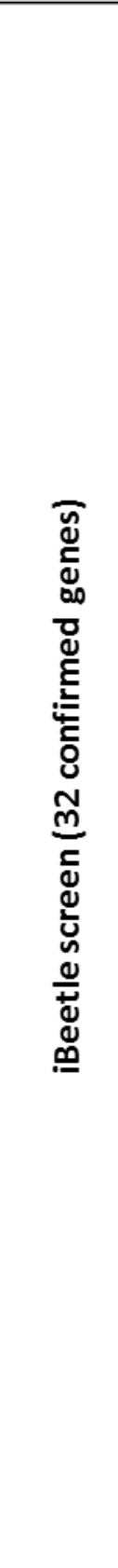 } & Tc_000240 & $11 \%$ & $8 \%$ & $39 \%$ & $28 \%$ \\
\hline & Tc_000379 & $177 \%$ & $119 \%$ & $98 \%$ & $106 \%$ \\
\hline & Tc_000504 & $166 \%$ & $140 \%$ & $119 \%$ & $169 \%$ \\
\hline & Tc_000885 & $0 \%$ & $0 \%$ & $14 \%$ & $12 \%$ \\
\hline & Tc_002616 & $226 \%$ & $170 \%$ & $99 \%$ & $123 \%$ \\
\hline & Tc_002723 & $90 \%$ & $66 \%$ & $125 \%$ & $160 \%$ \\
\hline & Tc_003409 & $41 \%$ & $40 \%$ & $46 \%$ & $44 \%$ \\
\hline & Tc_004129 & $123 \%$ & $99 \%$ & $87 \%$ & $119 \%$ \\
\hline & Tc_005167 & $0 \%$ & $0 \%$ & $10 \%$ & $16 \%$ \\
\hline & Tc_006408 & $111 \%$ & $88 \%$ & $113 \%$ & $144 \%$ \\
\hline & Tc_008608 & $189 \%$ & $149 \%$ & $75 \%$ & $63 \%$ \\
\hline & TC_010251 & $0 \%$ & $0 \%$ & $102 \%$ & $150 \%$ \\
\hline & Tc_011075 & $259 \%$ & $211 \%$ & $135 \%$ & $195 \%$ \\
\hline & Tc_011288 & $35 \%$ & $25 \%$ & $81 \%$ & $92 \%$ \\
\hline & Tc_011812 & $0 \%$ & $0 \%$ & $114 \%$ & $127 \%$ \\
\hline & Tc_012539 & $0 \%$ & $0 \%$ & $34 \%$ & $32 \%$ \\
\hline & Tc_012610 & $28 \%$ & $20 \%$ & $37 \%$ & $33 \%$ \\
\hline & Tc_012828 & $99 \%$ & $57 \%$ & $54 \%$ & $69 \%$ \\
\hline & Tc_012834 & $157 \%$ & $101 \%$ & $102 \%$ & $85 \%$ \\
\hline & Tc_013513 & $0 \%$ & $0 \%$ & $7 \%$ & $0 \%$ \\
\hline & Tc_013892 & $33 \%$ & $19 \%$ & $64 \%$ & $107 \%$ \\
\hline & Tc_014494 & $140 \%$ & $93 \%$ & $86 \%$ & $110 \%$ \\
\hline & Tc_014520 & $216 \%$ & $187 \%$ & $123 \%$ & $154 \%$ \\
\hline & Tc_014870 & $0 \%$ & $0 \%$ & $18 \%$ & $39 \%$ \\
\hline & Tc_014887 & $185 \%$ & $124 \%$ & $123 \%$ & $181 \%$ \\
\hline & Tc_015095 & $101 \%$ & $46 \%$ & $102 \%$ & $110 \%$ \\
\hline & Tc_015165 & $122 \%$ & $91 \%$ & $99 \%$ & $146 \%$ \\
\hline & Tc_015203 & $123 \%$ & $83 \%$ & $149 \%$ & $183 \%$ \\
\hline & Tc_015379 & $36 \%$ & $38 \%$ & $65 \%$ & $64 \%$ \\
\hline & Tc_015811 & $173 \%$ & $110 \%$ & $135 \%$ & $210 \%$ \\
\hline & Tc_015818 & $0 \%$ & $0 \%$ & $95 \%$ & $130 \%$ \\
\hline & Tc_016254 & $108 \%$ & $85 \%$ & $98 \%$ & 103 \\
\hline
\end{tabular}

\begin{tabular}{ll}
\hline $0 \%$ & substance not present \\
$1-50 \%$ & strongly reduced \\
$51-75 \%$ & reduced \\
$>76 \%$ & not-reduced, $w t=100 \%$
\end{tabular}


RESULTS
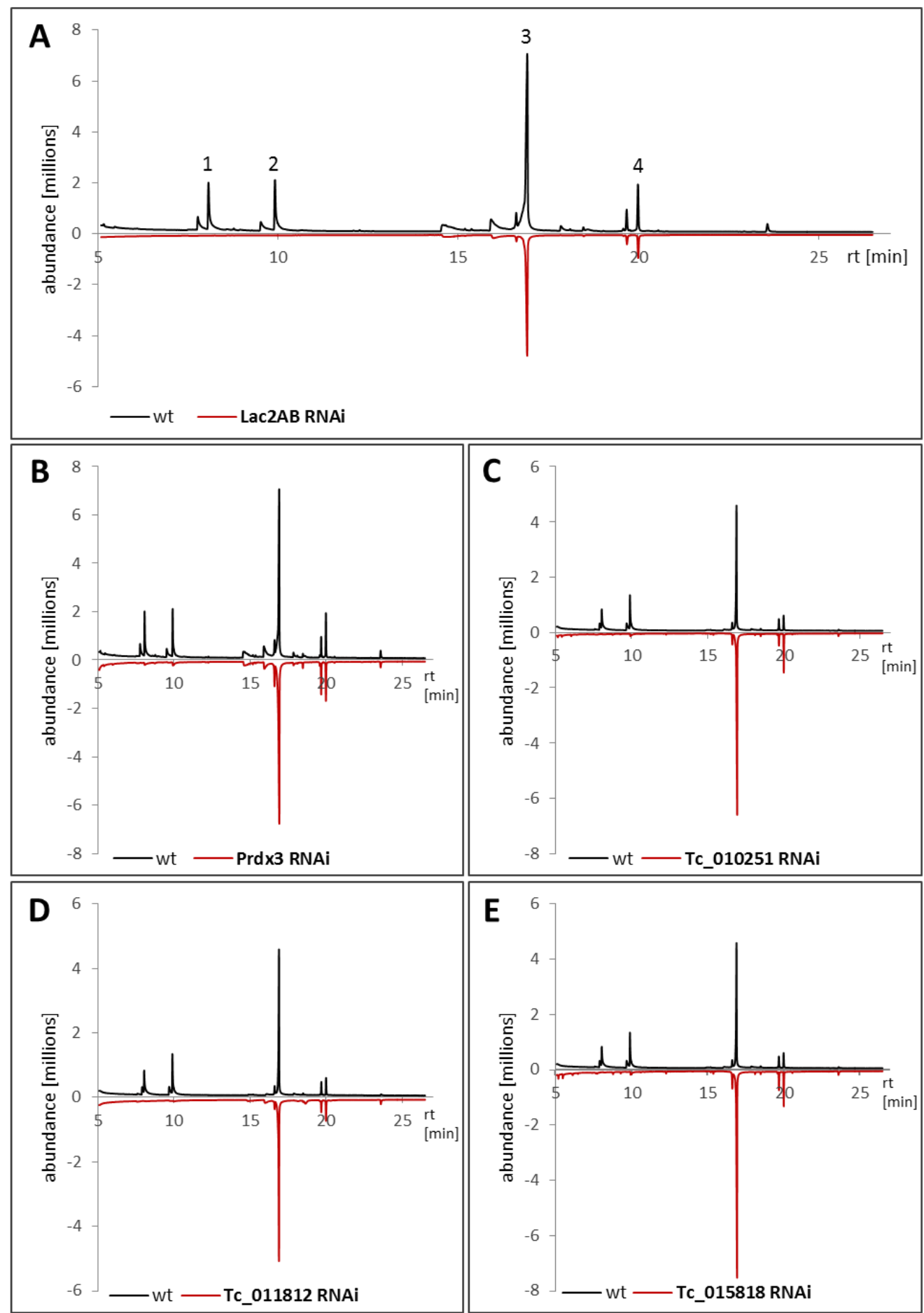

Figure 22: Gas chromatograms of stink gland content. The four main volatile substances detected via GC-MS in abdominal glands of wildtype beetles (wt, black line) are 1: 2-methyl-1,4-benzoquinone, 2: 2ethyl-1,4-benzoquinone, 3: 1-pentadecene, and 4: 1-heptadecene. In the knockdowns (red lines, for better comparison plotted as negative values), the benzoquinones (peaks 1 and 2) are missing. Note that different wt chromatograms are due to the measurement of two $(C-E)$ and three $(A, B)$ beetles per sample, respectively. rt: retention time. 


\section{RESULTS}

To check whether GC-MS phenotypes were more likely for genes within a distinct morphology phenotype category, the distribution of the 14 genes with GC-MS phenotypes was analyzed (Figure 23). At least one gene with altered gland chemical composition was represented in each of the seven phenotype categories. 'Colorless secretion', 'melanized content' and empty/necrotic' phenotypes were strongly represented in the gland volatile-affecting genes. A correlation between morphological gland phenotype and altered gland content was also shown for genes with 'irregular reservoir size', 'less secretion', and 'secretion color darker'.

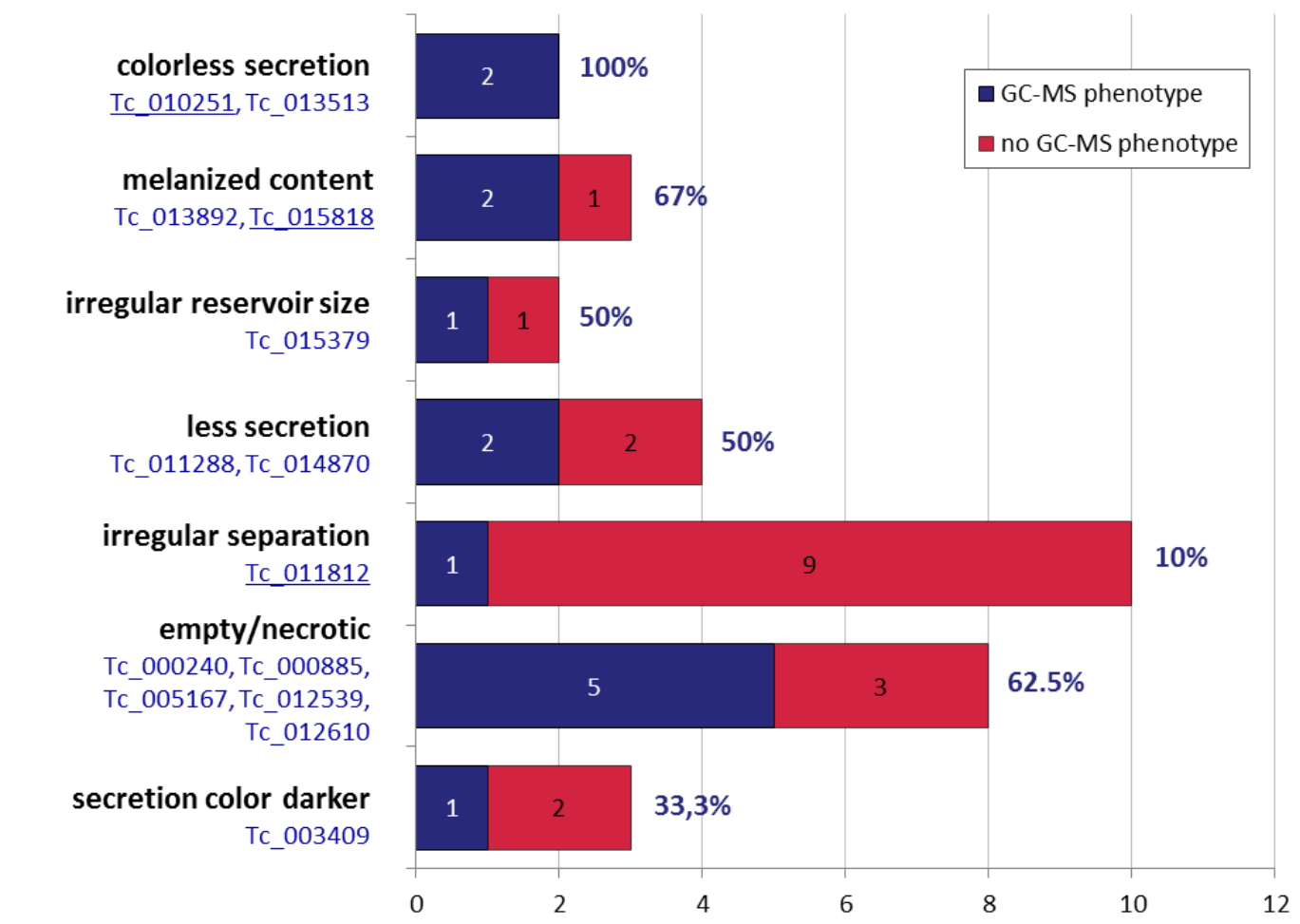

Figure 23: Distribution of 14 genes with GC-MS phenotype compared to their gland morphology phenotype categories. Each bar represents the total number of genes belonging to the respective categories. In blue and white font color the number of genes with GC-MS phenotype within a group is depicted, whereas genes with no altered gland volatiles are marked by red and black font color. Blue percentages next to the bars refer to GC-MS phenotype genes within the respective category. Genes with a GC-MS phenotype are listed with their OGS number in blue. The three benzoquinone-less genes are underlined.

However, 'irregular separation' phenotypes were found to seldom go along with changes in composition of gland volatiles. The three benzoquinone-less genes also stem from three different phenotype categories ('colorless secretion', 'melanized gland content' and 'irregular separation'), supporting the assumption that there is not only a single phenotype category 
related to altered gland secretion composition. It should be noted that for both genes, which were identified with a colorless secretion phenotype upon knockdown, the GC-MS analysis of knockdown gland contents revealed a lack of benzoquinones. This finding may relate the yellow color of wildtype gland secretion to the presence of benzoquinones. For candidates from the enhancer trap screen and selected glucosidases, phenoloxidases, and peroxidases from the transcriptome data, gland contents of three male and three female beetles per sample were analyzed separately with GC-MS, enabling the detection of possible gender-specific differences in terms of volatile secretion composition changes. Measurements were performed once and the results are displayed in Table 5. Similar to Table 4, abundances for each substance was calculated as percentage of the respective wildtype abundance, whereby $100 \%$ indicated wildtype level of a substance, $>100 \%$ an increase and $<100 \%$ a reduction of the respective compound compared to wildtype levels. In fact, both enhancer trap screen-identified genes (Tc_007045 and Tc_013894) demonstrated gender-specific GC-MS phenotypes. Tc_007045 RNAi resulted in no alkenes in female glands, whereas the knockdown of Tc_013894 led to no detectable BQ's and alkenes in males and reduced levels in females, except for 1-heptadecene. For the knockdown of the four glucosidases, a wildtypic volatile composition was obtained, being in line with the finding that the respective glands showed no morphological alterations (data not shown). The RNAi-mediated knockdown of Tyr1 caused reduced levels of benzoquinones but did not alter abundances of the alkenes. The knockdown affecting both Lac2 splicing variants at a time $(\operatorname{Lac} 2 A B)$ resulted in no benzoquinones but less affected alkene levels in male beetles (chromatogram in Figure 22A), whilst in females neither benzoquinones nor alkenes were detected. Surprisingly, compound abundances in males and females were the opposite in the Lac2A knockdown situation. Male Lac2A RNAi looked like female $\operatorname{Lac} 2 A B$ knockdown and the female Lac2A knockdown was very similar to male $\operatorname{Lac} 2 A B$. Notably, the knockdown of splicing variant B gave the same result as the double knockdown in males, but for females the levels for benzoquinones and alkenes were only reduced to about half of the wildtype levels. Thus, Lac2 was designated as another benzoquinone-less gene. Secretion lacking benzoquinones were also found in $\operatorname{Prdx} 3$ knockdown glands (Figure 22B). Therefore Prdx3 was also ranked as a benzoquinone-less gene. 


\section{RESULTS}

Table 5: GC-MS analysis of candidates from the enhancer trap screen and the transcriptome data analysis. Measurements were performed for abdominal and prothoracic glands separately, but only the data for abdominal gland volatiles are depicted. Each measured sample contained the gland content of three beetles of the same gender. Per knocked-out gene, gland volatile composition was determined for male and female beetles. Abundances for each compound is calculated in percent of the respective wildtype abundance $(100 \%=w t, 50 \%=$ half the amount of wt). Black fields mark benzoquinone-less genes (BQ-less), gray fields accentuate genes with milder GC-MS phenotype. Genes with both affected BQ- and alkene levels are highlighted in red. GC-MS phenotypes of genes marked additionally with an asterisk seem to be gender-specific in part or completely. $M B Q: 2$-methyl-1,4-benzoquinone, $E B Q:$ 2ethyl-1,4-benzoquinone , 1-C15: 1-pentadecene, 1-C17: 1-heptadecene.

\begin{tabular}{|c|c|c|c|c|c|c|c|c|c|c|}
\hline \multirow{2}{*}{\multicolumn{2}{|c|}{ screen }} & \multirow{2}{*}{ gene } & \multicolumn{4}{|c|}{ male } & \multicolumn{4}{|c|}{ female } \\
\hline & & & $M B Q$ & $E B Q$ & $1-C 15$ & $1-C 17$ & $M B Q$ & $E B Q$ & $1-C 15$ & $1-C 17$ \\
\hline \multirow{2}{*}{ 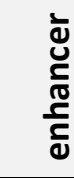 } & \multirow{2}{*}{$\frac{0}{\sqrt[T]{2}}$} & Tc_007045* & $96 \%$ & $93 \%$ & $85 \%$ & $59 \%$ & $10 \%$ & $9 \%$ & $31 \%$ & $16 \%$ \\
\hline & & Tc_013894* & $0 \%$ & $0 \%$ & $0 \%$ & $0 \%$ & $33 \%$ & $27 \%$ & $8 \%$ & $93 \%$ \\
\hline \multirow{9}{*}{ 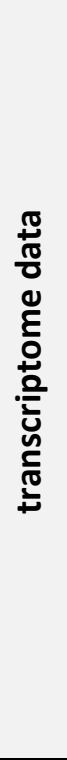 } & \multirow{6}{*}{ 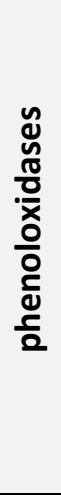 } & Tc_HEX1a & $79 \%$ & $82 \%$ & $89 \%$ & $79 \%$ & $86 \%$ & $97 \%$ & $73 \%$ & $50 \%$ \\
\hline & & Tc_Lac1 & $97 \%$ & $91 \%$ & $95 \%$ & $87 \%$ & $70 \%$ & $78 \%$ & $69 \%$ & $45 \%$ \\
\hline & & Lac2AB* & $0 \%$ & $0 \%$ & $67 \%$ & $51 \%$ & $0 \%$ & $0 \%$ & $2 \%$ & $0 \%$ \\
\hline & & Lac2A & $0 \%$ & $0 \%$ & $17 \%$ & $9 \%$ & $0 \%$ & $0 \%$ & $58 \%$ & $27 \%$ \\
\hline & & Lac2B & $9 \%$ & $10 \%$ & $68 \%$ & $59 \%$ & $41 \%$ & $42 \%$ & $53 \%$ & $34 \%$ \\
\hline & & Tyr1 & $12 \%$ & $13 \%$ & $87 \%$ & $87 \%$ & $31 \%$ & $27 \%$ & $91 \%$ & $84 \%$ \\
\hline & \multirow{3}{*}{ 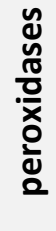 } & Tc_010355 & $59 \%$ & $46 \%$ & $69 \%$ & $57 \%$ & $37 \%$ & $33 \%$ & $57 \%$ & $32 \%$ \\
\hline & & Tc_010362 & $112 \%$ & $122 \%$ & $94 \%$ & $94 \%$ & $112 \%$ & $122 \%$ & $94 \%$ & $95 \%$ \\
\hline & & Prdx3 & $0 \%$ & $0 \%$ & $65 \%$ & $49 \%$ & $11 \%$ & $10 \%$ & $93 \%$ & $93 \%$ \\
\hline & & & & & & & \multicolumn{3}{|c|}{ substance not present } & $0 \%$ \\
\hline & & & & & & & \multirow{2}{*}{\multicolumn{3}{|c|}{$\begin{array}{r}\text { strongly reduced } \\
\text { reduced }\end{array}$}} & $1-50 \%$ \\
\hline & & & & & & & & & & $51-75 \%$ \\
\hline & & & & & & & \multicolumn{3}{|c|}{ not-reduced, wt $=100 \%$} & $>76 \%$ \\
\hline
\end{tabular}


RESULTS

\section{4 iBeetle and enhancer trap screen versus transcriptomics}

The three different approaches provided a broad spectrum of genes with potential roles in stink gland biology. To determine the effectiveness and utility of laborious and time-consuming genetic screens in contrast to rather easily and fast obtained RNA-seq data for differential expression analysis, the candidate genes identified with a gland phenotype in the iBeetle screen and in the enhancer trap screen were inspected for their expression levels in gland tissue (Figure 24). From the 34 discovered genes (32 iBeetle +2 enhancer trap) only five were highly differentially expressed in gland tissue (cut-off: 4-fold enrichment), comprising two of the BQless genes (Tc_010251 and Tc_011812), another gene that displayed a strong GC-MS phenotype (TC_013513), one with only mild alterations of gland volatile content (TC_015379) and one gene with morphological but no gland volatile phenotype (TC_000504). Surprisingly, gland expression levels of Tc_015818, one of the BQ-less genes, were not increased but very similar to those of the reference tissue. Notably, eight genes that also revealed very strong changes in gland secretion composition upon knockdown in the GC-MS analysis were found to be even strongly underrepresented in gland tissue, particularly Tc_014870. In sum, more than 85\% (29 of 34 genes) of potential candidate genes identified in the genetic screens would have been missed by a selection based on differential expression data alone, clearly demonstrating the utility and necessity of phenotypic screens for entire studies of stink gland biology.

A detailed examination about how many highly differentially expressed genes in gland tissue might have been missed in the iBeetle screen will be done after the end of the second iBeetle screening phase and will be presented elsewhere. At present it can be stated that Lac2 and Prdx3 were not detected in the iBeetle screen. For the knockdown of Lac2 in the iBeetle screen, high larval lethality and unfulfilled pupal eclosure have been annotated, so that no animal reached the respective adult stage (40 days after hatching) for stink gland examination. In contrast, Prdx3 larval injections led to analyzable adult beetles. However, the gland phenotype that was detected for Prdx3 knockdown in this study (less light yellow secretion, Figure 19B) has not been recognized in iBeetle despite being tested. As the two enhancer trap genes Tc_007045 and Tc_013894 were not included in the first round of iBeetle screening, so far no statement can be made about their recovery. 


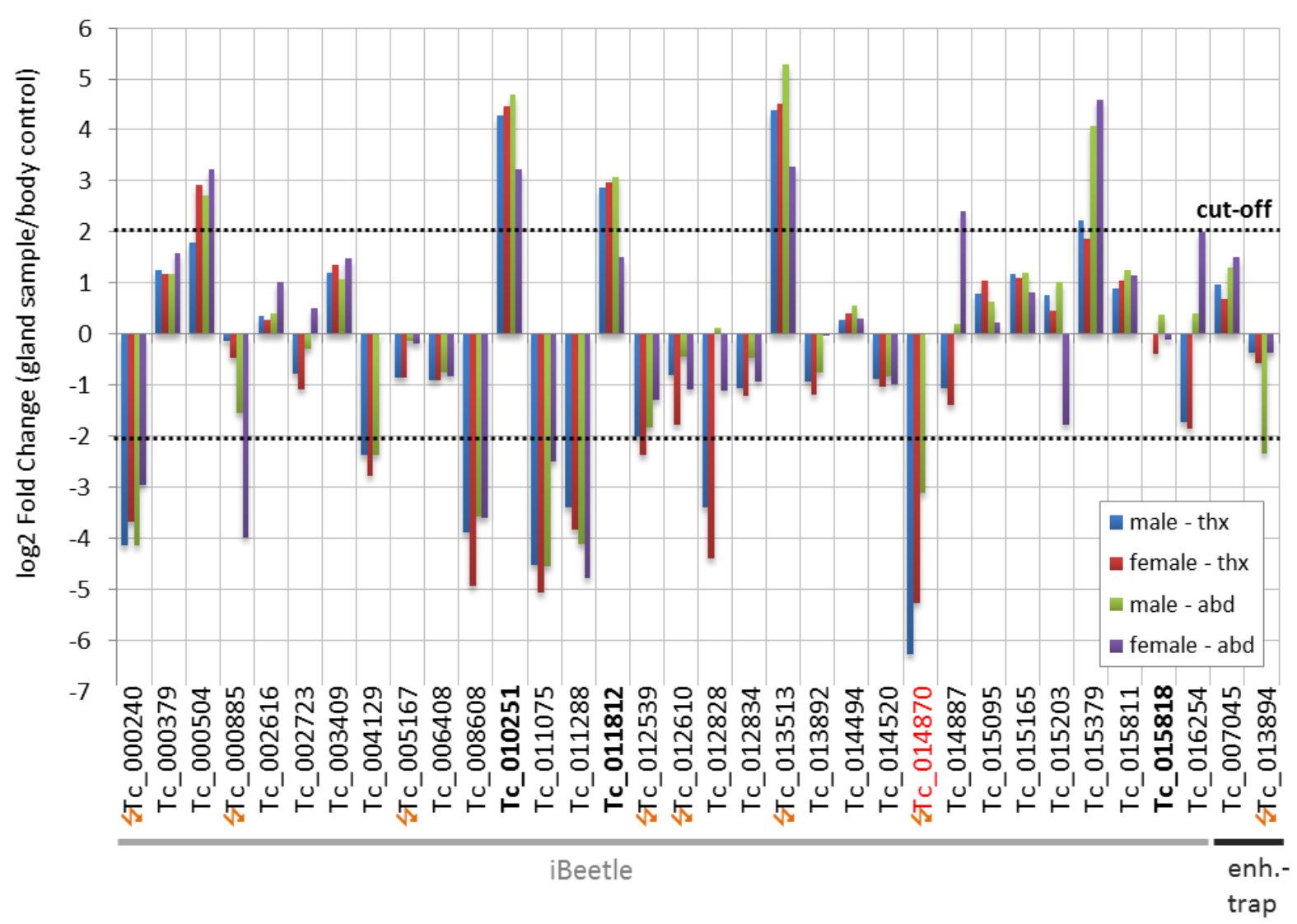

Figure 24: Differential expression levels of iBeetle and enhancer trap screen identified candidates. iBeetle genes are indicated by the gray line, the two enhancer trap candidates by the black line. In bold letters the three benzoquinone-less genes are highlighted, whereas orange flashes mark the eight other genes with strong gland phenotypes. In red a gene with strong reduced differential expression level despite of a striking GC-MS phenotype is demonstrated exemplarily. The corresponding dataset is available in the appendix (7.2: Table 12+13). abd: abdominal gland, thx: prothoracic gland, black dotted line: cut-off log2 FC of +2 and -2 (= 4-fold enriched or reduced).

\subsection{Localization of candidate gene expression in gland cells}

\subsubsection{Expression analysis via in situ hybridization}

First, fluorescence in situ hybridization (FISH) was performed to detect expression of the three BQ-less genes Tc_010251, Tc_011812 and Tc_015818 (performed by bachelor student Tobias Vollmer). However, the high background staining of cuticle and signal detection in no probe controls didn't allow us to make reliable statements (data not shown). Thus, in the following in situ hybridizations NBT/BCIP staining was used to detect expression patterns. As a proof of principle this method was first tested with two RNA probes that have been used successfully in 
gland FISH before by Jianwei Li. The first probe targeted Tcas-al P450 (GT12), a member of the cytochrome p450 superfamily that revealed alkene-less phenotype in GC-MS analysis upon gene knockdown. Expression of this gene had been detected by FISH in cell-type 1 but not in cell-type 2 of abdominal glands. In prothoracic glands, GT12 expression had also been allocated to celltype 1 by misinterpretation, as prothoracic gland tissue just composes of cell-type 2 (see introduction 2.2.1; Figure 27 in Li, 2013). The second probe was directed against Tcas-q/ VTGI (GT39), encoding a vitellogenin-like protein with BQ-less phenotype upon gene knockdown. Its transcripts had been localized via FISH in both abdominal gland cell-types and in type 2 cells of prothoracic glands (Li et al., 2013). Using the NBT/BCIP staining method for ISH detection in this study, the expression of GT12 in cell-type 1 only could be confirmed, whereas expression analysis for prothoracic glands revealed a restriction to cell $2 \mathrm{~b}$ (Figure 25B1+B2).

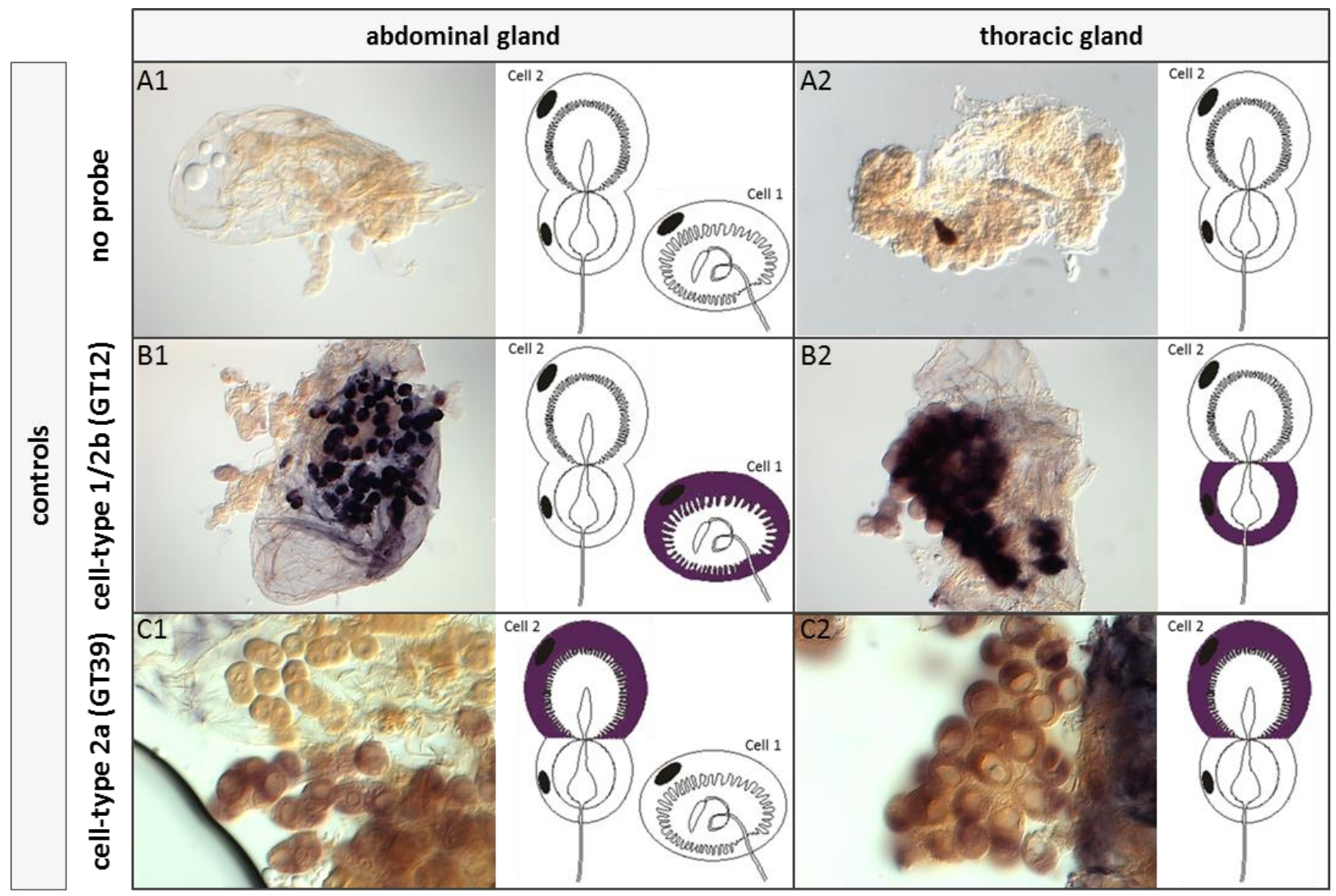




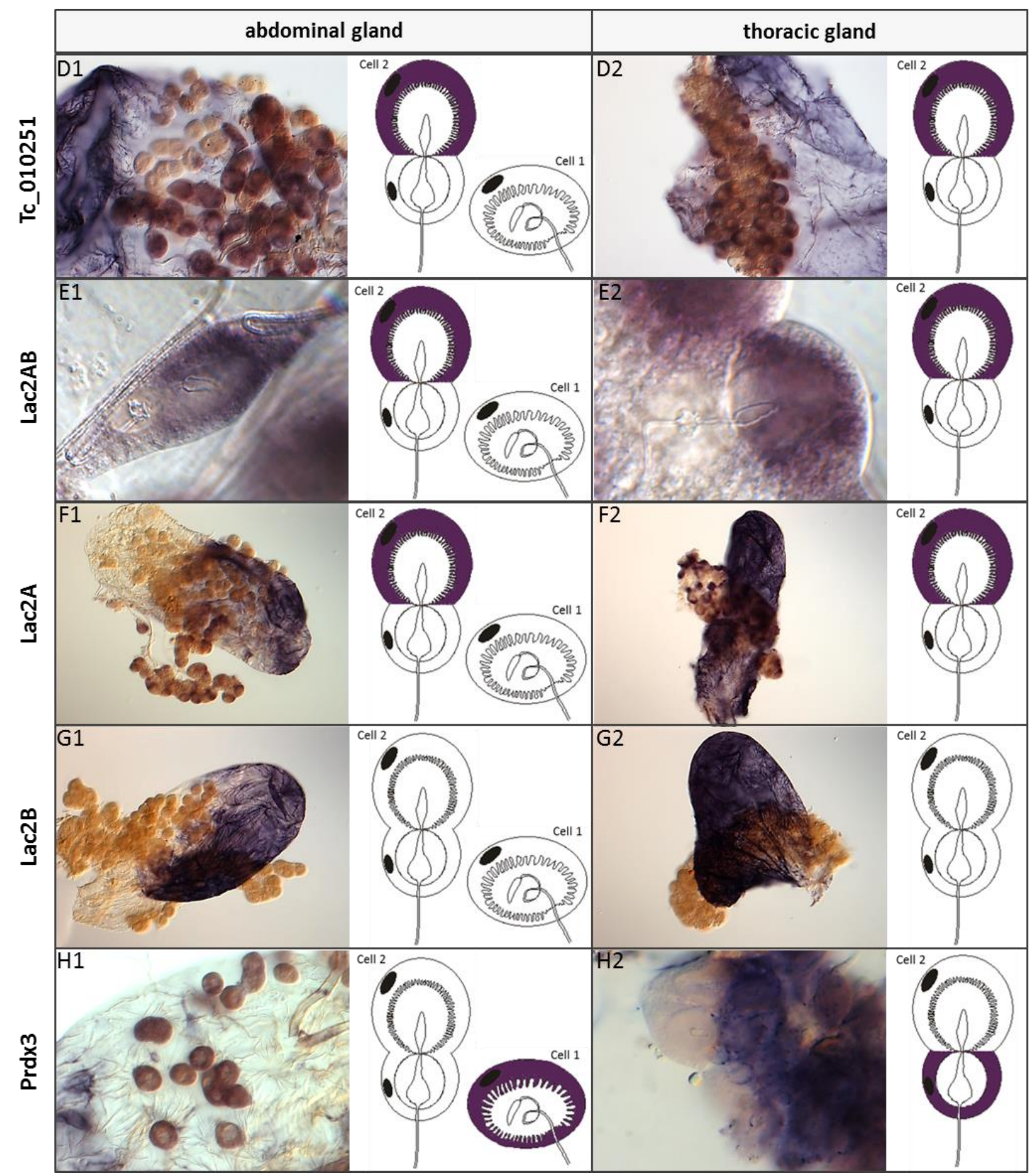

Figure 25: Gland in situ hybridization of selected genes. Expression analysis was done for abdominal (panels 1) and prothoracic glands (panels 2). Observations are summarized in particular schemes to the right. A: no probe as negative control, B: GT12 as positive control for cell-type 1 staining in abdominal glands and cell2b in prothoracic glands, C: GT39 as marker for cell-type 2a. D-H: probes against three BQless genes (Tc_010251, Lac2 and Prdx3), whereby $\mathbf{F}$ and $\mathbf{G}$ display ISHs of Lac2 splicing variants $\mathrm{A}$ and $\mathrm{B}$, respectively. Note that in contrast to all other pictures, E1 and E2 each show a close-up of a single type 2 cell. 
RESULTS

Li's FISH results for GT39 expression could be verified partly, as in abdominal and prothoracic glands only cells 2 a were stained, instead of reported whole type 2 cells and type 1 cells in abdominal glands (Figure $25 \mathrm{C} 1+\mathrm{C} 2$ ). Taken together, the newly established gland ISH protocol with NBT/BCIP detection provided clearer gland tissue staining with less background compared to Li's gland FISH protocol, enabling the study of expression patterns in more detail. With GT12 (cell-type 1 and 2b in abdominal and prothoracic glands, respectively) and GT39 (cell-type 2a in both gland types) as positive controls, the expression patterns of the five BQ-less genes (Tc_010251, Tc_011812, Tc_015818, Lac2, and Prdx3) and three genes with a strong GC-MS phenotype (Tc_000885, Tc_013513, and Tc_013894) were examined via gland ISH. Clear signals were obtained only for three BQ-less genes (TC_010251, Lac2, and Prdx3). Corresponding gland pictures are given in Figure 25D-H. Tc_010251 and Lac2AB were expressed in cell 2a in both gland types, just like the BQ-less GT39 (Figure 25C-E). In addition, Lac2AB (probe directed against both splicing variants) ISHs were also performed with probes targeting the splicing variants $A$ and $B$ separately (Figure $25 \mathrm{~F}+\mathrm{G}$ ). Whereas $L a c 2 A$ expression was similar to $L a c 2 A B$, no signal could be detected for splicing variant B, suggesting Lac2A as the major variant of Lac2 in stink gland tissue, which is also reflected by estimated read counts for the two splicing variants (Table 6).

Table 6: Extrapolation of reads for the two Lac2 splicing variants. Given that both variants are similar in total length and that exons 1-5 resemble almost the same length as the alternative exon sets 6-8 in each variant, and assuming an equally distributed read coverage, Lac2 reads can be estimated as follows: reads $($ Lac2A $)=$ reads $\left(T C_{-}\right.$010489) - reads $\left(T C_{-}\right.$010490) and reads $($Lac2B $)=$reads $\left(T C_{-}\right.$010490) $x 2$.

\begin{tabular}{|c|r|r|r|r|}
\cline { 2 - 5 } \multicolumn{1}{c|}{} & \multicolumn{4}{c|}{ reads } \\
\hline sample & Tc_010489 & Tc_010490 & \multicolumn{1}{c|}{ Lac2A } & \multicolumn{1}{c|}{ Lac2B } \\
\hline \hline male - thx & 85,149 & 183 & 84,966 & 366 \\
\hline female - thx & 105,542 & 197 & 105,345 & 394 \\
\hline male - abd & 139,482 & 206 & 139,276 & 412 \\
\hline female - abd & 35,974 & 55 & 35,919 & 110 \\
\hline
\end{tabular}




\section{RESULTS}

In contrast to $L a c 2 A / A B$ and Tc_010251, BQ-less Prdx3 showed the same expression pattern as the alkene-less GT12, namely cell-type 1 in abdominal glands and cell $2 \mathrm{~b}$ in prothoracic glands (Figure 25H1+H2). For the remaining five genes Tc_011812, Tc_015818, Tc_000885, Tc_013513, and Tc_013894 no staining was observed (data not shown). To find an explanation for that, read counts of genes with and without detectable signals were compared (Table 7). Noticeably, reads of detected genes were much higher than those of undetected genes. An exception is Tc_Prdx3, which showed a clear detection pattern despite the lower read count of 8000 . Thus, failed staining was much likely not due to technical issues but to the rather low sensitivity of the used detection method.

Table 7: Gland tissue read counts of genes selected for in situ hybridization. Genes above the red line displayed clear staining in the ISH, whereas for all genes below this line no specific expression patterns could be detected.

\begin{tabular}{|r|r|r|r|r|}
\hline \multirow{2}{*}{ gene } & \multicolumn{4}{|c|}{ reads } \\
\cline { 2 - 5 } & male - thx & female - thx & male - abd & female - abd \\
\hline GT12 (Tc_003768) & 539,533 & 641,884 & 463,311 & 129,261 \\
\hline GT39 (TC_011211) & 46,017 & 47,146 & 52,445 & 16,140 \\
\hline Prdx3 & 8,537 & 9,570 & 8,048 & 3,967 \\
\hline Tc_010251 & 29,710 & 33,432 & 39,562 & 14,185 \\
\hline Tc_010489 & 85,149 & 105,542 & 139,482 & 35,974 \\
\hline Tc_000885 & 144 & 113 & 54 & 10 \\
\hline Tc_010490 & 183 & 197 & 206 & 55 \\
\hline Tc_011812 & 7,031 & 7,559 & 8,061 & 2,731 \\
\hline Tc_013513 & 444 & 484 & 826 & 204 \\
\hline Tc_013894 & 2,425 & 2,095 & 609 & 2,408 \\
\hline Tc_015818 & 1,563 & 1,182 & 2,019 & 1,442 \\
\hline
\end{tabular}

\subsubsection{Subcellular localization of Lac2 protein}

Signal peptide analysis of both Lac2 proteins revealed a 24 amino acid long signal peptide sequence, targeting Lac2 for the secretion pathway (Figure 26). To determine the protein's place of activity in gland cells and subcellular structures, immunohistochemical detection of Lac2 was performed on whole dissected glands. Antisera against An. gambiae Lac2 (AgLac2) and 
RESULTS

M. sexta Lac2 (MsLac2) as well as affinity-purified MsLac2 polyclonal antibodies have been kindly provided by the group of Professor Michael Kanost, Kansas State University, USA. Both Lac2 antibodies (MsLac2 and AgLac2) have previously been shown to detect Tribolium Lac2 protein in immunoblots (Dittmer et al., 2009 and statement by Prof. Michael Kanost). However, their functioning in animal tissue was not examined before.

\begin{tabular}{|c|c|c|c|c|c|c|c|}
\hline Name & Len & mTP & SP & other & Loc & RC & TPlen \\
\hline TcLac2A & 717 & 0.049 & 0.917 & 0.055 & $\mathbf{s}$ & 1 & 24 \\
\hline TcLac2B & 713 & 0.048 & 0.916 & 0.056 & $\mathrm{~s}$ & 1 & 24 \\
\hline cutoff & & 0.000 & 0.000 & 0.000 & & & \\
\hline
\end{tabular}

Figure 26: Signal peptide analysis and subcellular location prediction of Lac2 protein. Prediction was performed with the TargetP 1.1 server for TcLac2A (AY884061) and TcLac2B (AY884062). Both splicing variants were predicted with a 24 amino acid long signal peptide (blue box) and therefore as targeted for the secretory pathway (red box). Loc: prediction of localization ( $\mathbf{S}=$ secretory pathway), RC: Reliability class, from 1 to 5 , where 1 indicates the strongest prediction. TPlen: predicted presequence length. $\mathbf{m T P}$, SP and other are scores on which the final prediction is based. For detailed explanation see http://www.cbs.dtu.dk/services/TargetP-1.1/output.php.

Incubation of abdominal glands with pre-immune sera of the rabbits that were subsequently immunized with epitopes of MsLac2 (Figure 27B1) and AgLac2 (Figure 27B2) respectively, revealed varying grades of signals from the cytoplasm of both cell-types as well as the presence of vesicle-like structures on the cell surface - preferentially of cell-type $2 b$ and to a less frequency on type-1 cells. In prothoracic glands pre-immune sera detected less in the cytoplasm but huge amounts of small vesicles around cell-type $2 b$, filling the intercellular space (Figure 27B3+4). All signals obtained from pre-immune sera incubation were designated as background. 


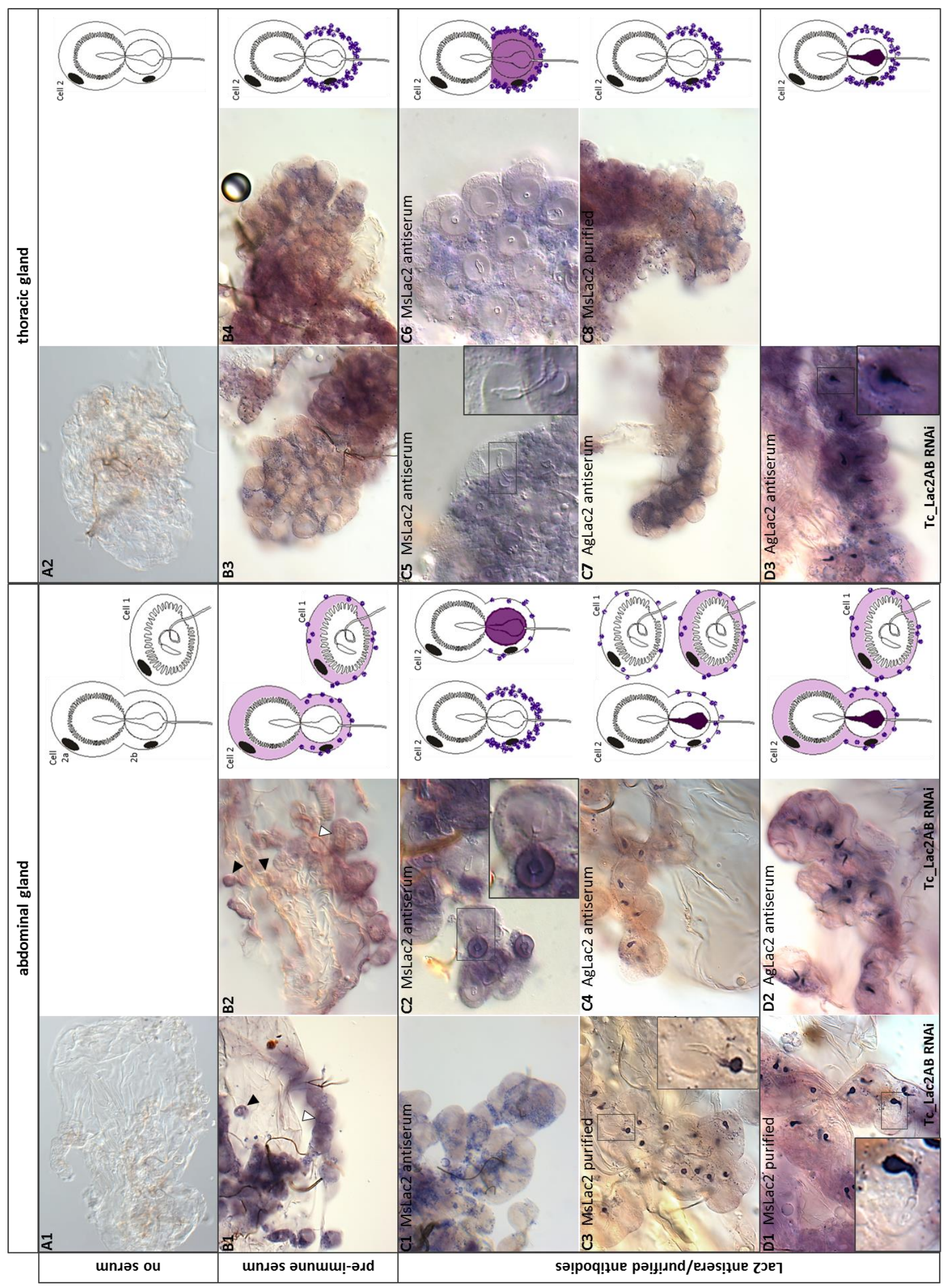


Figure 27: Subcellular localization of Laccase 2 protein in Tribolium stink glands. Immunohistochemical staining was performed on wildtype (A-C) and Lac2AB-RNAi knockdown (D) dissected abdominal and prothoracic stink glands of ten days-old beetles (left and right panels, respectively). Similar staining patterns were also found for 20 and 40 days-old beetles (not shown). The used polyclonal Lac2 antisera were: $M$. sexta Lac2 antiserum $(\mathbf{C} \mathbf{1}+\mathbf{2}, \mathbf{C} 5+\mathbf{6})$, its Lac2 specific purified antibodies alone $(\mathbf{C} \mathbf{3}, \mathbf{C} \mathbf{8}, \mathbf{D} \mathbf{1})$ and An. gambiae Lac2 antiserum $(\mathbf{C 4}, \mathbf{C 7}, \mathbf{D 2}+\mathbf{3})$. No serum as well as respective rabbit pre-immune sera served as negative controls (panels $\mathbf{A}$ and $\mathbf{B}, \mathbf{B} \mathbf{1 + 3}$ from rabbit afterwards immunized with MsLac2, B2+4 from AgLac2 antibody producing rabbit; black triangles point to type-1 cells, white triangles mark exemplary cell-type 2 bunches). Most striking findings are summarized in schemes to the right. Note that no pictures of type- 1 cells are shown, because they only displayed varied grades of background staining (as indicated in the schemes).

In the following incubations with MsLac2- and AgLac2 antisera (Figure 27C), three different staining patterns were observed: Most glands showed only background staining with varying intensities of signals in the cytoplasm and number of outer vesicles (Figure 27C1+C6-8). But for approximately one out of five glands additional and strong signals were observed from cell-type $2 \mathrm{~b}$. Either the whole $2 \mathrm{~b}$ vesicle including the lumen of the vesicular organelle and their outlining were stained (Figure $27 \mathrm{C} 2+5$ ), or strong signaling was restricted to the lumen of the vesicular organelle in $2 b$ vesicle (Figure $27 \mathrm{C} 3+4$ ). Both staining patterns were found in differently aged beetles (10 days, 20 days and 40 days old) and seemed to be gender-independent (data not shown). Moreover, they could be obtained with both, AgLac2 and MsLac2, antibodies, indicating a Lac2 dependent signaling. Surprisingly, these signals remained in Lac2AB-RNAi knockdown situations (Figure 27D1+2), and even were detected on this situation for the first time in prothoracic glands (Figure 27D3 compared to C5-8). An incomplete Lac2 knockdown could be excluded as an explanation for that phenomenon, as the glands dissected for the immunehistochemical staining showed their characteristic colorless secretion like invented before. Thus, it may be stated with care that Lac2 accumulates in the vesicular organelle of $2 \mathrm{~b}$ gland cells.

\subsection{Enzyme activity assays}

Lac2 immunohistochemical staining suggested an accumulation of the protein in the vesicular organelle of $2 \mathrm{~b}$ cells (4.5.2). To validate the location of Lac2 protein within gland cells, two colorimetric assays were set up, showing the presence of active phenoloxidase in gland tissue by the formation of colored products, which first accumulate in gland tissue and then disperse in the surrounding staining solution. 


\section{RESULTS}

\subsubsection{ABTS assays on gland tissue}

In a first test, dissected abdominal and prothoracic stink glands of ten 15 days-old beetles were incubated in $1 \mathrm{ml}$ ABTS solution and product formation was recorded over a time period of 60 minutes by measuring the absorbance. Surprisingly, no product was formed (Figure 28A).

A

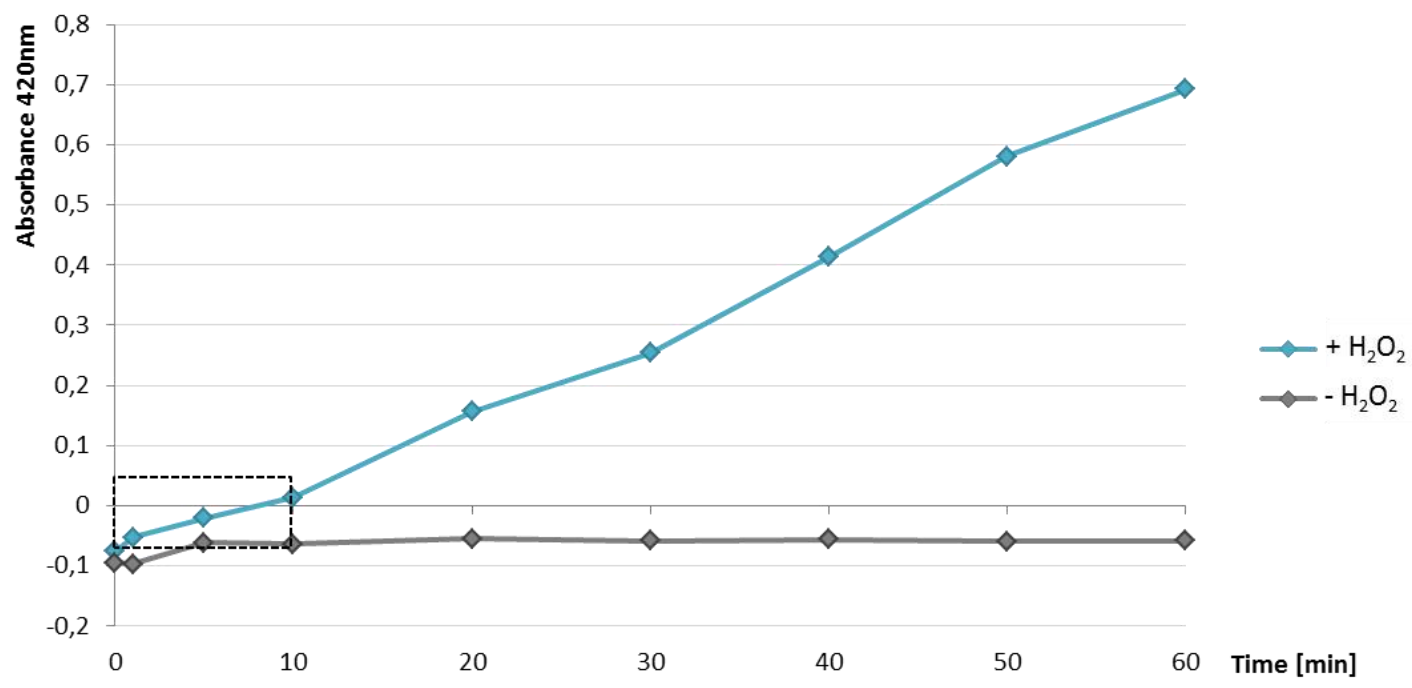

B

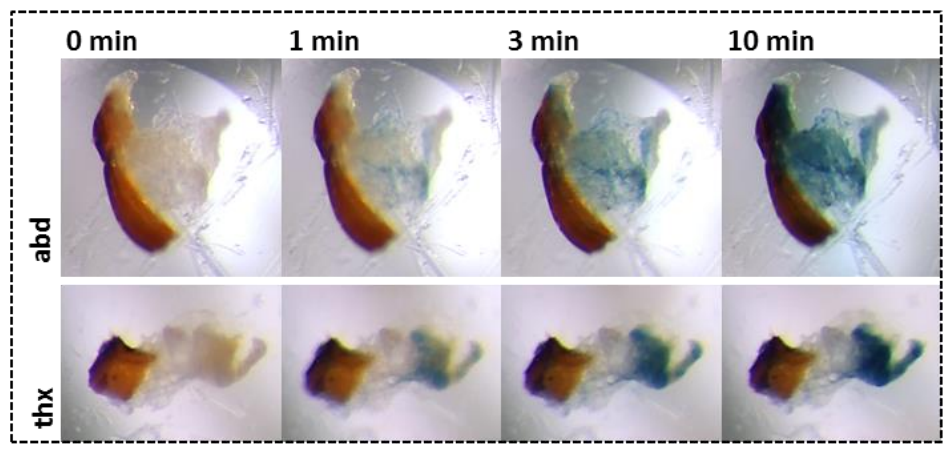

C

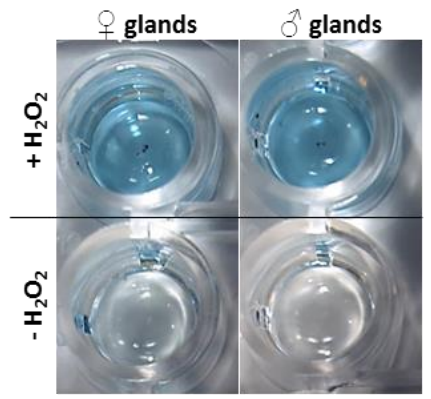

Figure 28: ABTS assay test-run. A Curve diagram showing absorbance in dependency of time for two reactions: blue line: presence of $\mathrm{H}_{2} \mathrm{O}_{2}$, gray line: no $\mathrm{H}_{2} \mathrm{O}_{2}$. Measurement was done with abdominal and prothoracic glands of ten beetles (15 days old). B Product formation in abdominal and prothoracic gland tissue in the first ten minutes of the ABTS assay (dashed black box). C Photograph of ABTS assay performed in well-plate, with glands of two beetles separated by gender.

However, repeating the same experiment in the presence of $\mathrm{H}_{2} \mathrm{O}_{2}$, the formation of ABTS cation radicals could be detected photometricly and was allocated to both gland types (Figure 28A+B). To check if the reaction without $\mathrm{H}_{2} \mathrm{O}_{2}$ might take longer time, the glands were incubated for up to $48 \mathrm{~h}$ but no product formation could be observed (data not shown). As the same $\mathrm{H}_{2} \mathrm{O}_{2}-$ 
dependent gland tissue activity was obtained in experiments with glands separated by gender (Figure 28C), this phenomenon appeared to be gender-independent. Considering that ABTS has been used successfully to demonstrate phenoloxidase activity many times in the past and even for Tribolium Lac2 (Dittmer et al., 2009; Gorman et al., 2012), three reasons were imaginable for why no activity showed up in the ABTS assay in this study.

First: Neither Lac2 nor any other enzyme with a phenoloxidase activity is present in the gland tissue. This would be the most obvious but least likely explanation at the same time, as (I) Lac2 transcripts and especially Lac2 proteins have been detected in gland cells of differently aged beetles via ISH and immunohistochemical staining, respectively (Figures $25+27$ ), and (II) the lack of Lac2 has been linked to a BQ-less gland phenotype (Figures 18D + 22A and Table 5) .

Second: Failed product formation is due to wrong reaction conditions in which the enzyme is inactive. However, substrate concentration, buffer composition and $\mathrm{pH}$ value as well as incubation time and -temperature have been selected according to previous laccase activity studies (Gorman et al., 2012; Hattori et al., 2005; Liang, 2006). As it was shown that the $\mathrm{pH}$ value is a critical parameter for T. castaneum Lac2 substrate specificity (Gorman et al., 2012), it was tested whether or not the $\mathrm{pH}$ value of the buffer system undergoes a noticeable change upon the application of gland material thereby prohibiting the reaction, but this was also not the case (not shown).

Third: Glandular Lac2 protein needs activation. Due to the findings of the ABTS assay, activation might be achieved directly through $\mathrm{H}_{2} \mathrm{O}_{2}$ or an $\mathrm{H}_{2} \mathrm{O}_{2}$-mediated interaction with another protein. If this would be the case, the product formation seen in the ABTS assay might be due to a peroxidase activity necessary to activate Lac2 or a peroxidase activity by itself. An ABTS assay using the purified recombinant Lac2A- and Lac2B enzymes instead of whole gland tissue (performed together with Maureen Gorman in the Department of Biochemistry and Molecular Biophysics at the Kansas State University, Manhattan Kansas), demonstrated that (I) purified enzymes do not need any activation to oxidize ABTS, and (II) the addition of hydrogen peroxide even led to a dose-dependent inhibition of the laccase activity (Figure 29). However, in vivo the situation might be different and the addition of hydrogen peroxide still might activate Lac2 by removing possible inhibitors. 


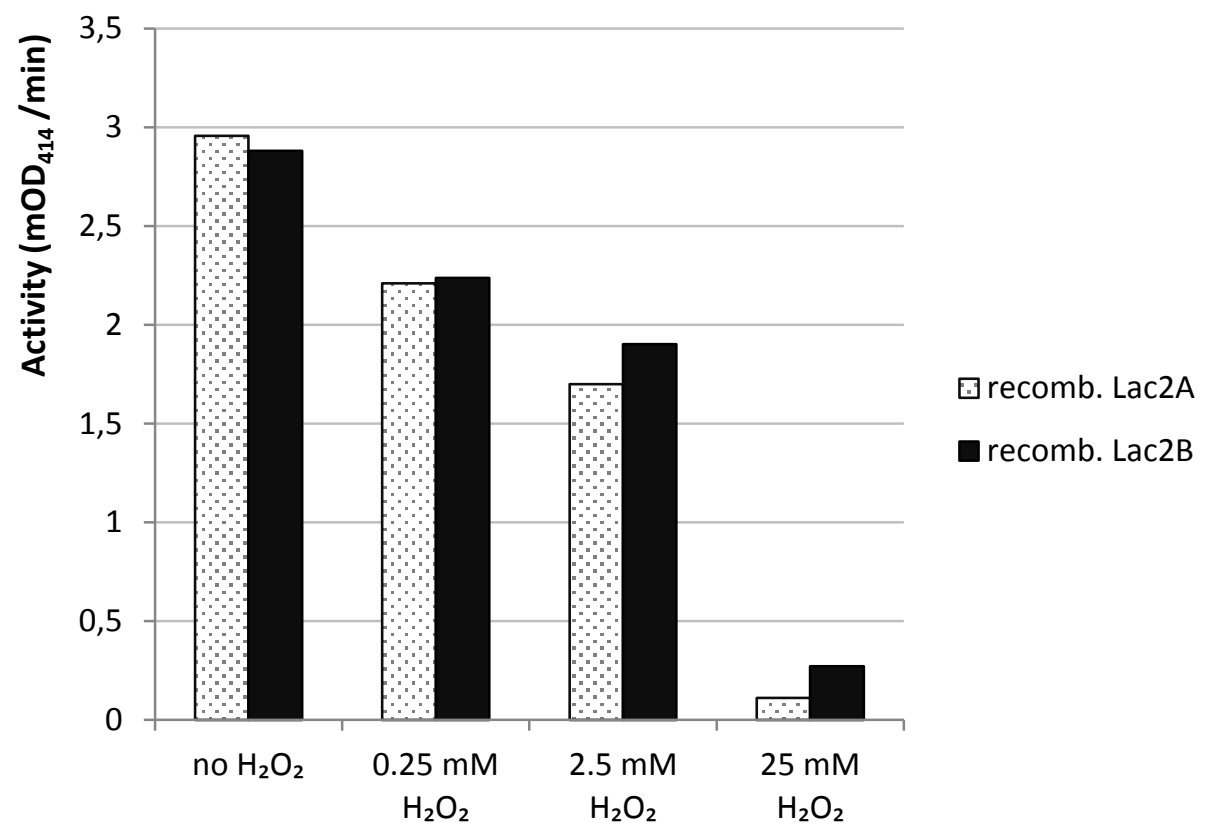

Figure 29: ABTS assay with purified recombinant Lac2 enzymes. The ABTS assay was performed with purified Lac2A (dotted) and Lac2B (black), respectively, in the absence or presence of indicated hydrogen peroxide concentrations. The activity of the enzymes is given as the median absorbance increase per minute.

Following this idea, in a next step the $\mathrm{H}_{2} \mathrm{O}_{2}$-dependent ABTS assay was performed for female abdominal gland, female prothoracic gland, male abdominal gland, and male prothoracic gland separately and at different beetle ages $(0,5,10,20$ and 40 days after hatching) to find optimal reaction conditions and to collect more characteristics of the reaction. In a total reaction volume of $100 \mu \mathrm{l}$, glands of two individuals were incubated and absorbance was measured after $60 \mathrm{~min}$. For each situation, five reactions were measured. Calculated mean values for absorbance of each reaction condition are presented in Figure 30. Note that high absorbance values were synonymously interpreted with high enzyme activity. In short, no gender-specific activity was observed, whereas significant differences existed between abdominal and prothoracic glands, as exemplarily indicated for A20. Noticeably, the highest activity was found in five days-old beetles for both gland types. Thus, in subsequent ABTS experiments, glands of five days-old beetles were used and abdominal and prothoracic glands were measured separately, whereas gender separation was not continued. 


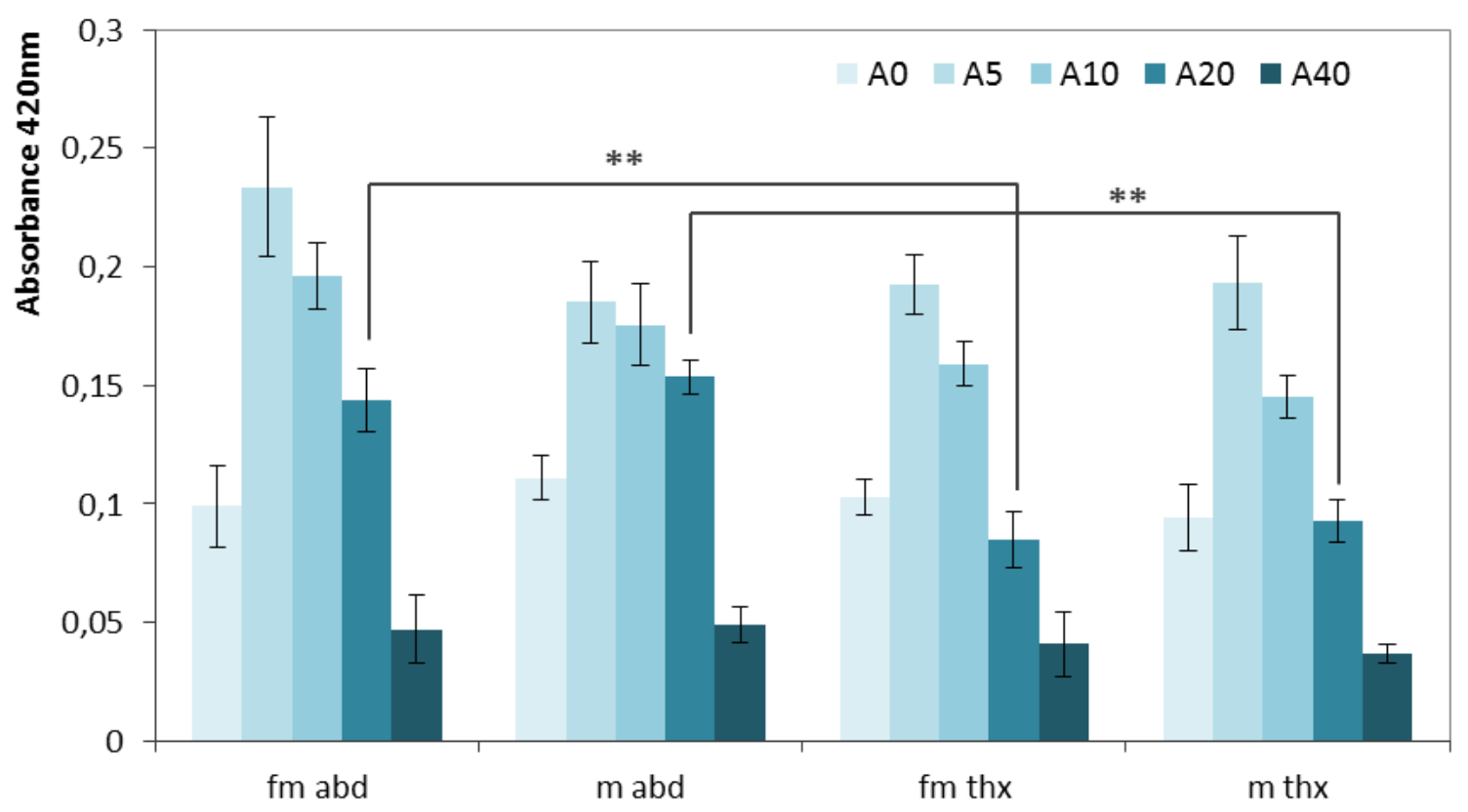

Figure 30: ABTS assay with glands of differently aged wildtype beetles. Differences in ABTS product formation are displayed in dependency of beetle age, gender and gland type. Significant differences are marked exemplarily for A20. A0, A5, etc. describe the age of the analyzed beetles, with 0: day of beetle's hatch, 5: five days after hatching and so forth. Statistical analysis was performed with student's t-test. ${ }^{*} p<0.05, * * p<0.01, * * * p<0.001$.

To get an idea in which gland cells the ABTS-based enzymatic activity appeared, microscopic pictures were taken of abdominal glands after the 60 min incubation in ABTS-solution with and without $\mathrm{H}_{2} \mathrm{O}_{2}$ (Figure 31). It should be noted that the proper turquois-green color (compare Figure 28B) rapidly turned to dark violet upon transfer of the glands from the well-plate to the microscope slide before it completely vanished within minutes. Nevertheless, enzymatic activity in the ABTS assay could be clearly observed in the cytoplasm of cell-type 1 (Figure 31A2) and with less intensity in the vesicle of $2 b$ cells as well as the outlining of its vesicular organelle (Figure 31A1), which partly conformed to the locus of Lac2 protein of the former immunostaining (Figure 27C2). Moreover, enzymatic activity was also allocated to the tubules (Figure 31A1), which connect type-2 cells with the reservoir, although it could not be clearly seen whether the product formed in the lumen of the tubules or in the cytoplasm of the tubule carrying cells. 

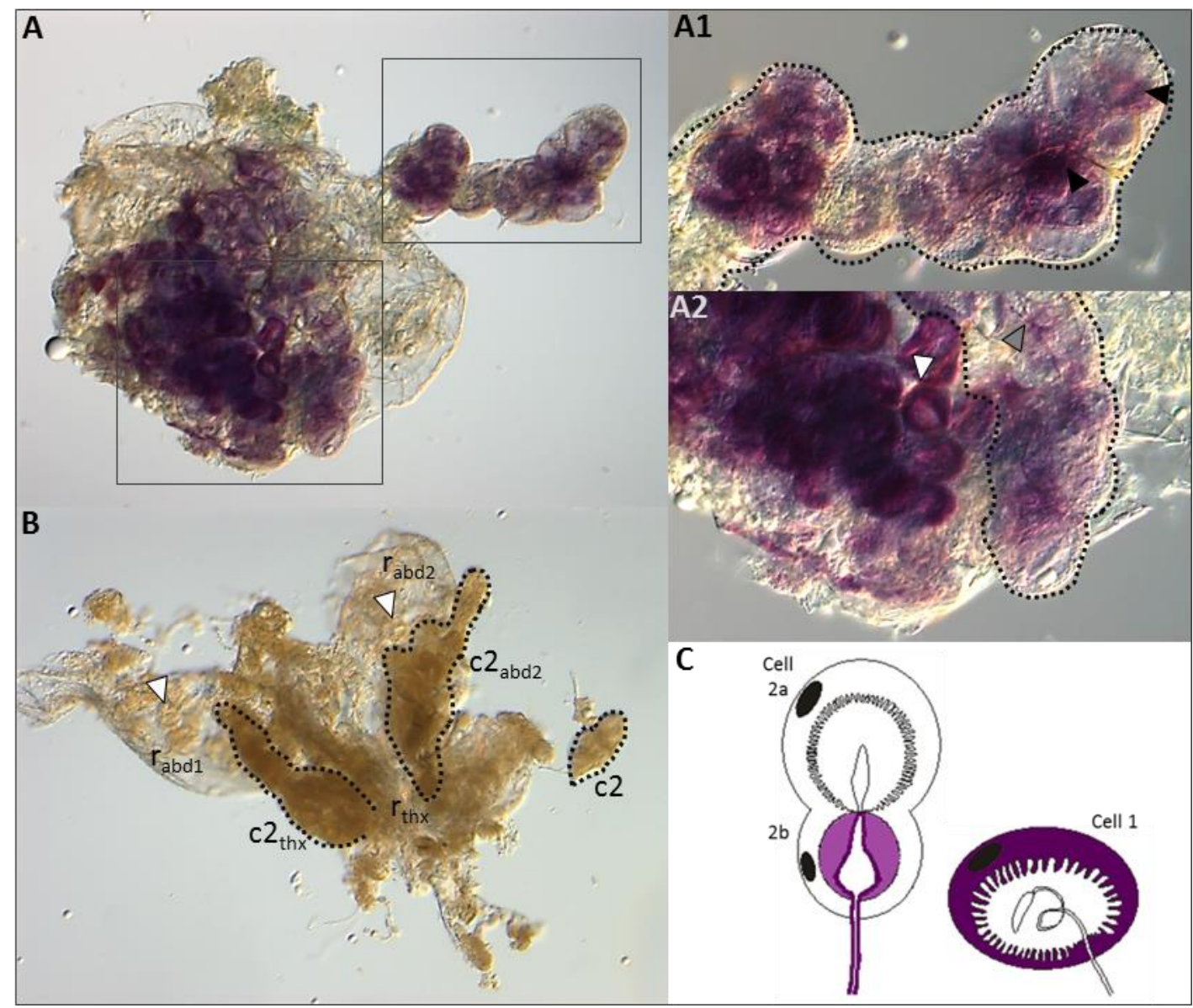

Figure 31: Microscopic pictures of glands after the ABTS assay. Pictures were taken after 60 minutes incubation in ABTS-solution with (A-A2) and without $\mathrm{H}_{2} \mathrm{O}_{2}(B$, negative control). Findings are summarized in a scheme (C). Note that in the negative control (B) a bunch of two abdominal glands and one prothoracic gland is shown. Type-2 cells are framed by dotted lines. White triangles point exemplarily to type- 1 cells, whereas black triangles mark stained $2 \mathrm{~b}$ cells and gray triangles indicate colored tubules of type-2 cells. r: reservoir, c2: cell-type 2 .

To check the hypothesis that Lac2, after being somehow activated by the presence of $\mathrm{H}_{2} \mathrm{O}_{2}$, plays a role in product formation of the ABTS assay, activity assays were performed with Lac2 knockdowns. In addition, Prdx3 knockdown glands were tested, as the detected enzymatic activity matched the locus of Prdx3 expression (cell-type 1; compare Figures $25 \mathrm{H}$ and 31 ). So potentially Prdx3 could be involved, although its predicted functional and spatial relation to mitochondria would not support this idea. For highest enzymatic activity, beetles analyzed now were five days-old (four male and four female per reaction) and abdominal and prothoracic glands were measured separately. The corresponding curve chart is given in Figure 32A. 
RESULTS

Strikingly, starting absorbance values (0min) differed highly. The reason for that was that the ABTS solution, which is per se slightly green before the start of the reaction, bleached upon application of gland material to different degrees. Choosing the blank with the same composition as a sample, namely $1 \mathrm{ml}$ ABTS solution plus glands of eight wildtype beetles but without adding $\mathrm{H}_{2} \mathrm{O}_{2}$ (as this would have started the color reaction), couldn't compensate this effect. Thus, for a better comparison of product formation in wildtype and knockdown glands, the overall absorbance alteration after 60 min was calculated for every situation and is displayed separately for abdominal and prothoracic glands in the bar chart in Figure 32B. It can be clearly seen that (I) in abdominal glands more product was formed than in prothoracic glands, (II) Prdx3 knockdown glands were as productive as wildtype glands, indicating that Prdx3 has no influence on the enzymatic activity, and (III) effects of Lac2 knockdowns were much more intense in abdominal glands compared to prothoracic glands. Having a closer look to product formation in abdominal glands, the knockdown of both Lac2 variants at the same time ( $L a c 2 A B$ RNAi) led to a drastic reduction. However, single knockdown of Lac2A showed almost no effect, whereas in Lac2B knockdown surprisingly no product was formed. The situation for prothoracic glands presented itself differently. Here, the double knockdown of Lac2 led to about $50 \%$ reduced product formation, while both single knockdowns reached product levels close to the double knockdown.

In summary, it can be stated that Lac 2 does have an influence on the enzymatic activity in the ABTS assay on gland tissue, however its nature remains completely elusive at this point. Together with unintentional but obvious peroxidase activities, triggered by the addition of hydrogen peroxide, it was concluded that this assay was not suitable to gain further insights in the location of active Lac2 in stink gland cells and therefore the following DOPA assay was tested instead. 
A
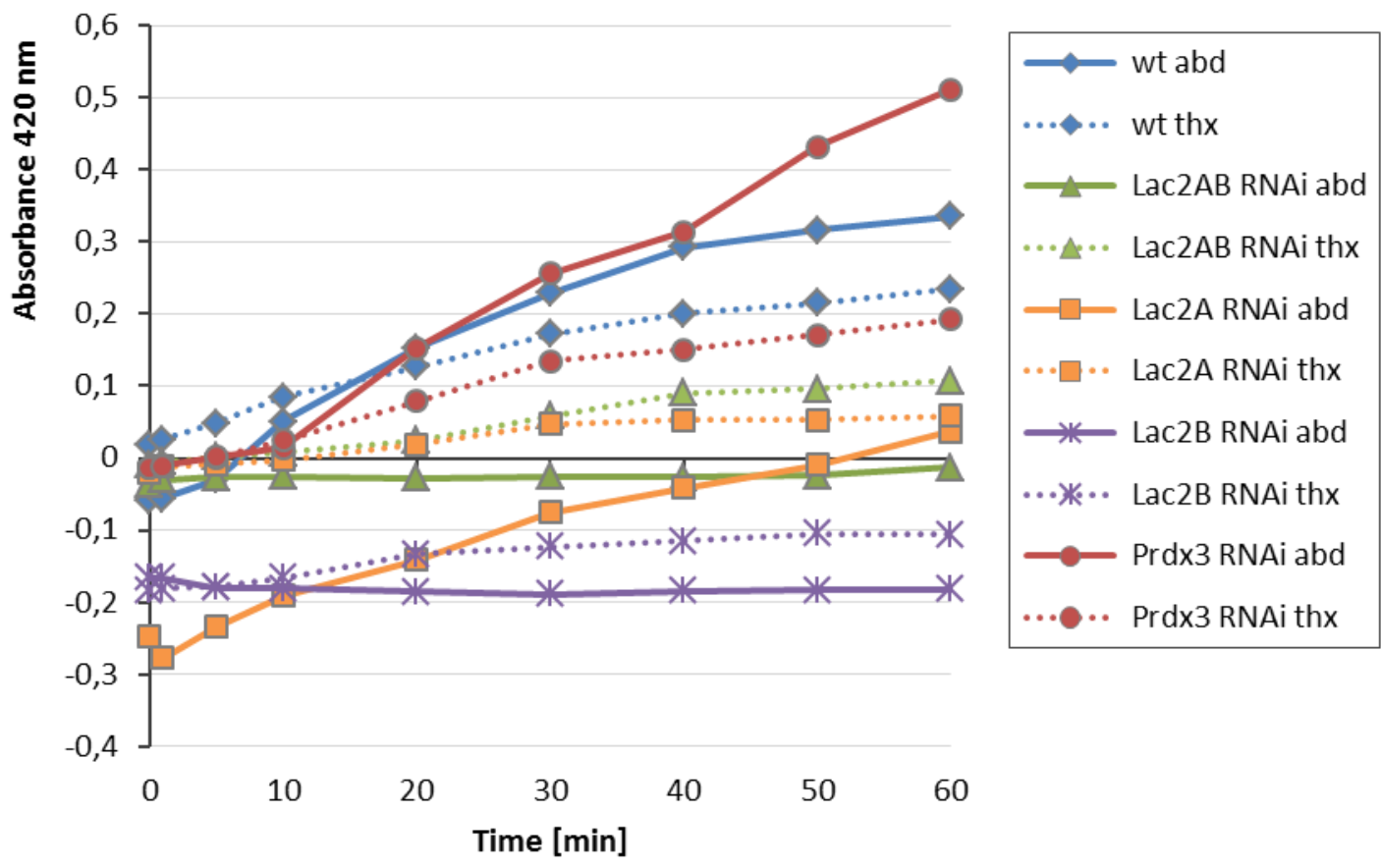

B

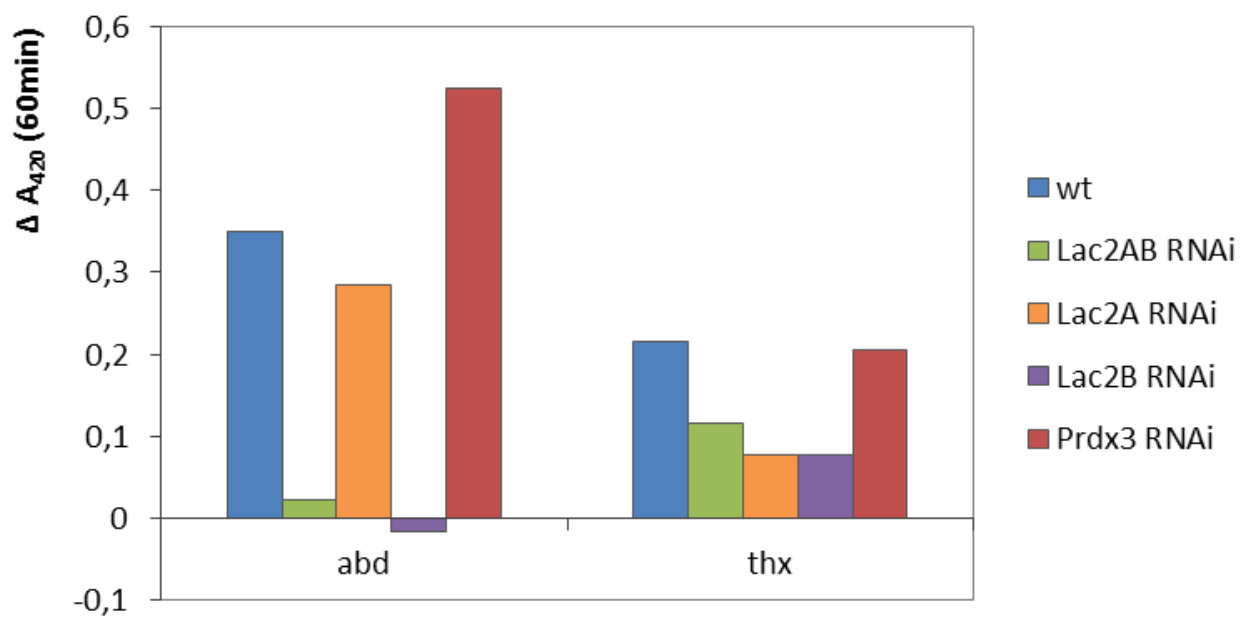

Figure 32: ABTS assay with Lac2 knockdowns. A Curve chart. Abdominal gland measurements are indicated by solid lines while prothoracic glands are displayed as dotted lines for wildtype and respective knockdown situations. B Bar chart showing absorbance alteration after $60 \mathrm{~min}$, displayed separately for abdominal and prothoracic glands. Note that abdominal and prothoracic glands were always taken from the same eight individuals per situation (wildtype or knockdown). 
RESULTS

\subsubsection{DOPA assays on gland tissue}

In a test run with abdominal and prothoracic glands of two male or two female beetles, it could be shown that the dopachrome formation worked independently of the addition of hydrogen peroxide and was not gender-specific, confirming that L-DOPA is a substrate to phenoloxidases but not to peroxidases (Figure 33A). Similarly to the ABTS assay, DOPA assays were then performed with beetles of different ages, namely at day of hatching and 5, 10, 20 and 40 days after hatching. For each age, glands were additionally separated by gender and gland type. Each of the four combinations of gland-type and gender was measured five times for every age in independent reactions. Mean values and standard errors of the mean were calculated and are depicted in Figure 33B.

A

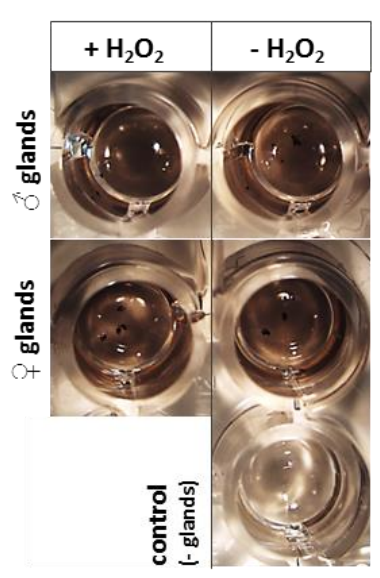

B

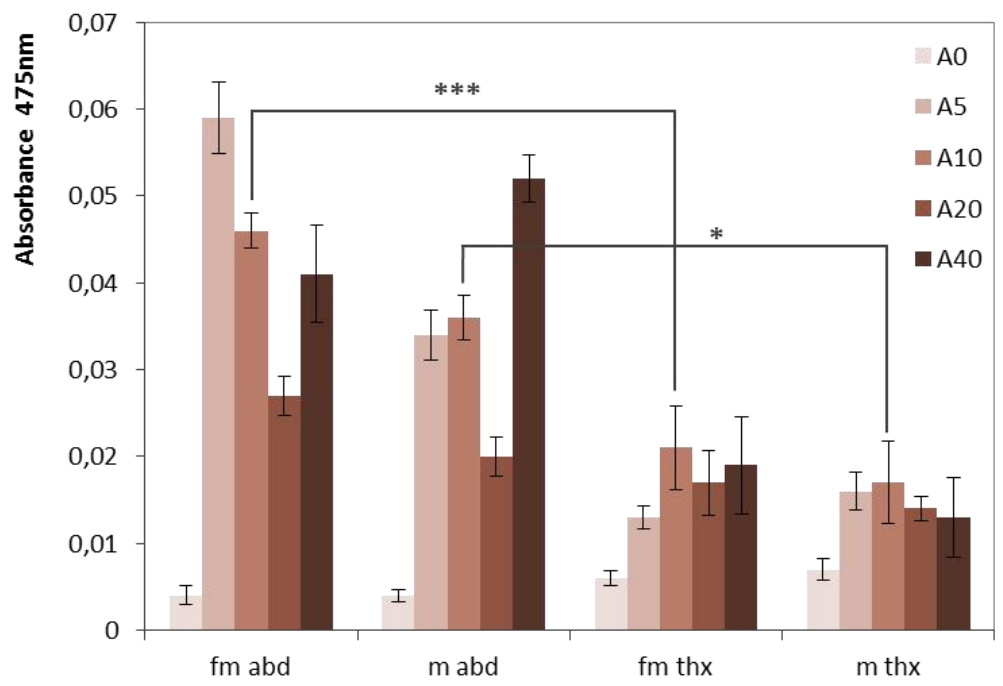

Figure 33: DOPA assays on wildtype gland tissue. A Picture of DOPA assay performed in well-plate with abdominal and prothoracic glands of two male or two female beetles per well with and without $\mathrm{H}_{2} \mathrm{O}_{2}$ (left and right, respectively). B Bar chart displays differences in dopachrome formation in dependency of beetle age, gender and gland type. Significant differences are marked exemplarily for A10. Age of the analyzed beetles is given in days after hatching with $A 0$ being the day of beetle's hatch. Statistical analysis was performed with student's t-test. ${ }^{*} p<0.05,{ }^{* *} p<0.01, * * * p<0.001$.

In agreement with the findings for the ABTS assay, product formation was higher in abdominal glands than in prothoracic glands. Also, significant differences in absorbance level could be found between gland-types, as exemplarily indicated for A10, but rather less variance was observed for the same gland-type in different genders. Highest level of dopachrome formation 


\section{RESULTS}

was obtained for gland tissue from five to ten days-old beetles. Interestingly, the enzymatic activity in abdominal gland tissue of 40 days-old beetles re-increased, after it had dropped significantly in 20 days-old beetles ( $\mathrm{fm}$ abd: $\mathrm{A} 10$ to A20 drop: $\mathrm{p}<0.001, \mathrm{~m}$ abd: $\mathrm{p}<0.05$, not indicated in Figure 33B).

To localize the enzymatic activity on subcellular level, microscopic pictures were taken of abdominal and prothoracic glands after $60 \mathrm{~min}$ incubation in L-DOPA solution. Findings for abdominal glands are presented in Figure 34. In short, dopachrome formation could not be observed in tubules (Figure 34A) or cell-type 1 (Figure 34B), but was linked to type-2 cells (Figure 34C1-F1). Interestingly, dopachrome formation was detected in different compartments of cell-type 2. It was found in dots in the cytoplasm of 2a cells (Figure 34C1), ubiquitously spread in vesicle 2a (Figure 34D1), in the lining of the vesicular organelle's head in 2a vesicle (Figure $34 \mathrm{E} 1$ ), as well as in the lining of the vesicular organelle part harbored in vesicle $2 \mathrm{~b}$ (Figure 34F1). Note that dopachrome patterns depicted in Figure $34 \mathrm{C}+\mathrm{D}+\mathrm{F}$ were also observed in prothoracic glands (data not shown). Importantly, in $L a c 2 A B$ knockdowns, dopachrome was neither formed in abdominal glands (Figure 34G1), nor in prothoracic glands (not shown), strongly indicating the dependency of the DOPA reaction on the presence of Lac2.

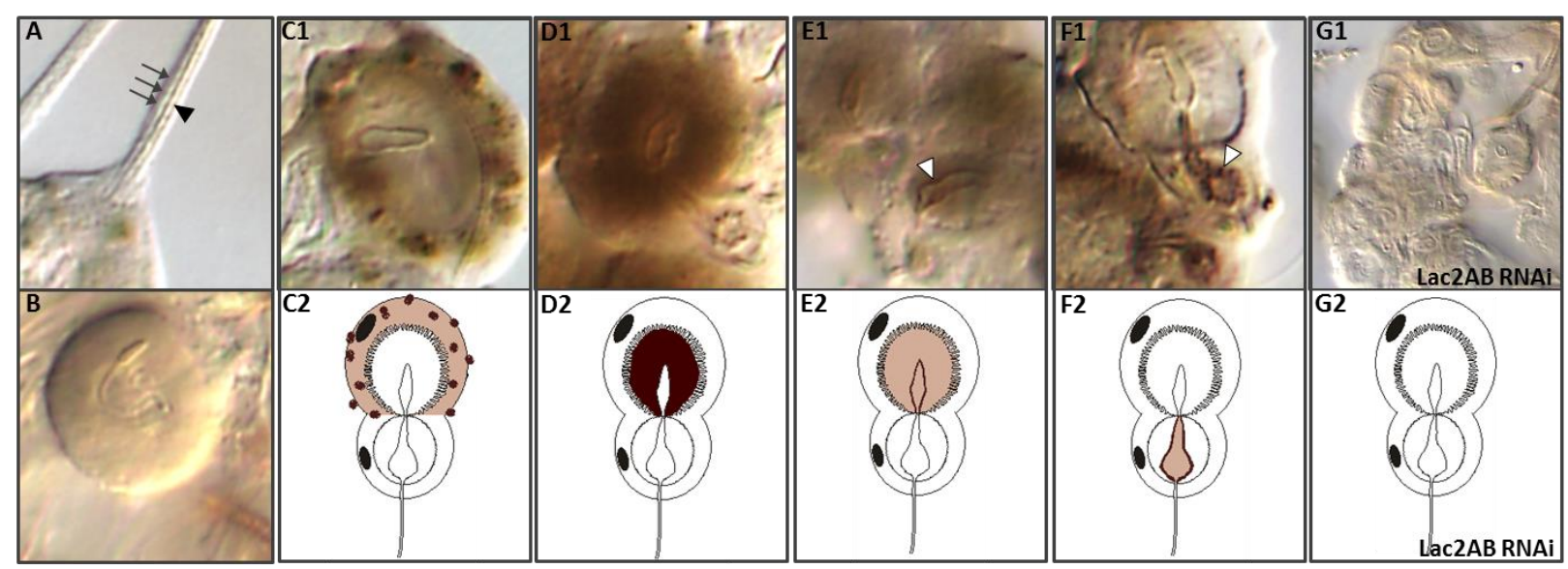

Figure 34: Microscopic pictures of abdominal glands. In A gray arrows mark a tubule carrying cell running off of a type- 2 cell, whereas the black triangle points to the tubule itself. B demonstrates a type- 1 cell with its vesicular organelle. Pictures C1-G1 were taken from abdominal glands incubated with L-DOPA solution for 60 minutes, whereby C1-F1 display wt glands and G1 a Lac2AB knockdown situation. White triangles in E1 and F1 point to brown colored outlining of vesicular organelle $2 a$ and $2 b$, respectively. Schemes C2-G2 summarize the findings. 
Subsequently, dopachrome formation was recorded in Lac2 knockdowns. Abdominal and prothoracic glands were measured separately and also the additional separation by gender was kept this time for a more detailed analysis. As the enzymatic activity could be allocated to type2 cells but not to cell-type 1 , no role for Prdx3 was expected and thus not tested, since $\operatorname{Prdx} 3$ transcripts have only been detected in cell-type 1 and were not predicted to be secreted (Figures $25+19 A)$. In addition to the Lac2 knockdowns, the influence of the phenoloxidase Tyr1 on dopachrome formation in stink gland tissue was analyzed. In vertebrates and invertebrates, this enzyme is crucial for melanin synthesis (Cerenius et al., 2010). It catalyzes the hydroxylation of tyrosine to DOPA and the oxidation of DOPA to dopaquinone, the latter being the reaction this DOPA assay is based on. As control, dsRNA of DsRed, encoding a red fluorescent protein from Discosoma sp., was injected to make sure that possible changes in enzymatic activity were not due to the dsRNA injection process itself but dependent on respective Tribolium gene knockdowns. In Figure 35, the enzymatic activity in different knockdown glands is given by the absorbance measured after $60 \mathrm{~min}$ incubation. In agreement with previous findings (Figure 32B), in general, absorbance levels were different between abdominal and prothoracic glands but quite similar for male and female beetles. Injection of DsRed dsRNA did not lead to major changes in absorbance level compared to wildtype. However, it should be mentioned that dopachrome formation was found to be significantly reduced in male prothoracic glands but at the same time significantly increased in male abdominal glands. Notably, upon knockdown of both $L a c 2$ variants at the same time ( $L a c 2 A B)$, the enzymatic activity was significantly and strongly reduced in abdominal and prothoracic gland tissue. This held also true for the single knockdown of splicing variant $\operatorname{Lac} 2 A$, though with less strength and except for male prothoracic gland, where no significant differences were observed in this case. In contrast, knockdown of splicing variant $\operatorname{Lac} 2 B$ did not lead to reduced dopachrome formation in any gland, but even to significantly increased absorbance levels compared to wildtype, suggesting that the oxidation of L-DOPA might be independent of Lac2B. The lack of Tyr1 resulted in less strong but significant reduction of dopachrome formation in abdominal glands, indicating the presence of active Tyr1 at least in abdominal gland cells and its involvement in dopachrome formation in this assay. But, as microscopic pictures of Lac2 knockdown glands already revealed that the main part of 


\section{RESULTS}

enzymatic activity was dependent on Lac2, only a minor role is suggested for Tyr1 in this assay, though microscopic pictures of Tyr1 knockdown glands are still to be analyzed.

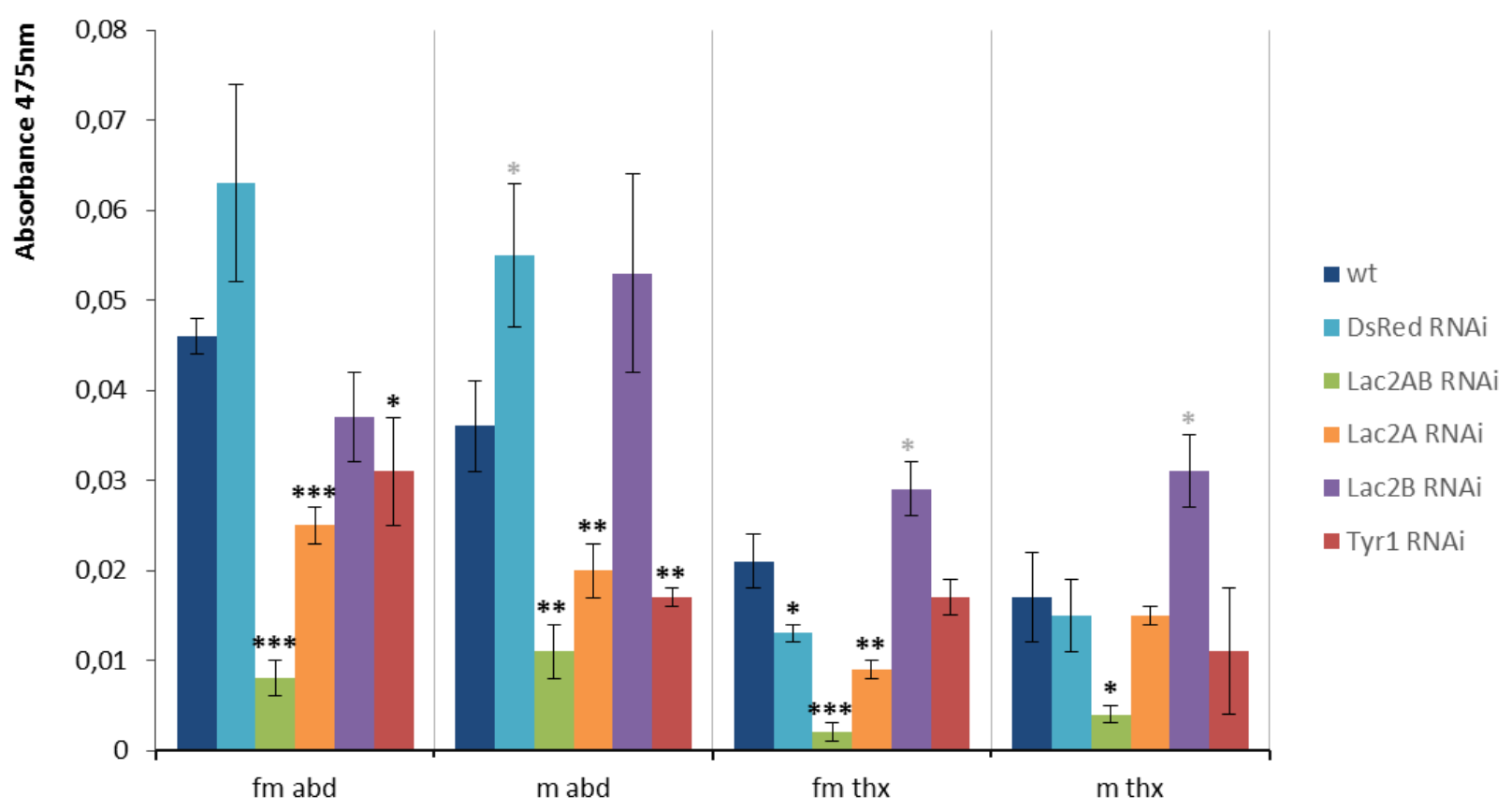

Figure 35: Dopachrome formation in 10 days-old knockdown beetles. Significant differences to wildtype glands are indicated by respective $p$-values, whereby black asterisks stand for significantly reduced levels of dopachrome formation and gray asterisks mark increased levels. Statistical analysis was performed with student's t-test. ${ }^{*} p<0.05,{ }^{* *} p<0.01, * * * p<0.001$.

In summary, enzymatic activity in the DOPA assay was obviously dependent on both tested phenoloxidases, Lac2 and Tyr1, but with Lac2 clearly playing the major role. Thus, this assay was suitable to study the subcellular location of Lac2 by means of its enzymatic activity loci. Referring to Figure 34, Lac2 does not only accumulate in vesicular organelle $2 b$, as identified previously by immunohistochemical staining, but is also present in other cell compartments, pointing to a possible secretion pathway that unusually ends in the lumen of the vesicular organelle of cell-type 2 a. 
RESULTS

\subsubsection{DOPA assay on gland secretion}

To test whether Lac2 is contained in its active form in the secretions stored in the reservoir, the DOPA assay was performed on abdominal gland secretions of five wildtype and five $L a c 2 A B$ RNAi knockdown beetles, respectively. As depicted in Figure 36, dopachrome was formed in wildtype gland secretion, indicating the presence of an active phenoloxidase. Compared to wildtype, dopachrome formation was strongly reduced in Lac2AB knockdown gland contents, demonstrating the dependency of dopachrome formation on the presence of Lac2. Thus, it can be concluded that Lac2 is not only located in cellular structures but also contained in the defensive secretions of stink glands in an active state.

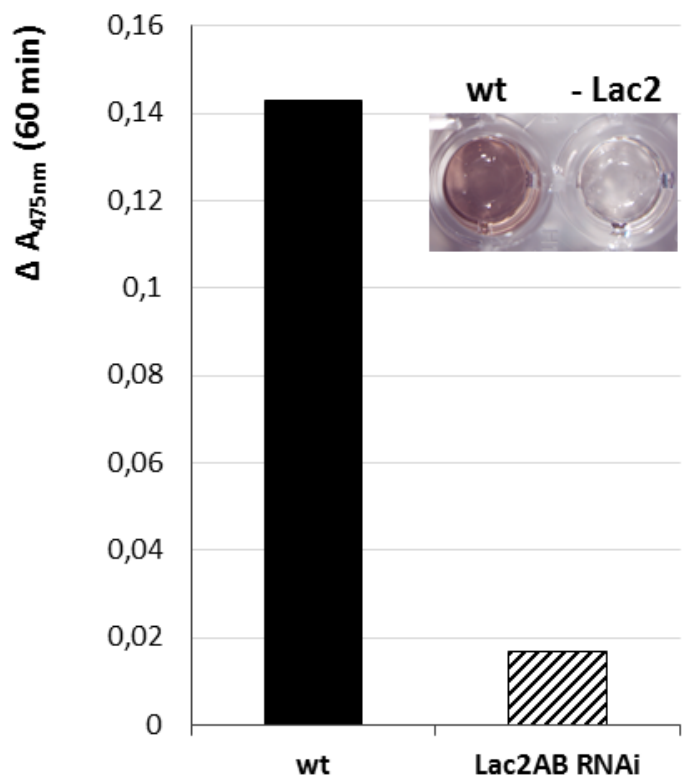

Figure 36: DOPA assay on gland secretions. Dopachrome formation was measured in secretions of wildtype (wt) and Lac2 knockdown (Lac2AB RNAi) abdominal glands of five individuals each, with depiction of the two reactions in the well-plate. 

DISCUSSION

\section{Discussion}

\subsection{Stink gland morphology of T. castaneum and E. longicollis is highly similar}

In the past, extensive studies on defensive glands in the family of tenebrionid beetles have been performed. Their morphology was analyzed to deduce phylogenetic relationships of the many groups within the Tenebrionidae (Tschinkel, 1975b; Tschinkel and Doyen, 1980), but most emphasis was put on the understanding of secretion production (Eisner et al., 1964; Happ, 1968; Roth, 1943; Tschinkel, 1969, 1975c). Detailed morphological microscopic observations of secretion producing cells of the tenebrionid beetles $E$. longicollis and $T$. castaneum have been summarized in a reference diagram by Happ (1968). In this study, microscopic examinations of T. castaneum stink glands confirmed the findings from Eisner et al. (1964) and Happ (1968) in terms of secretion-producing type 2 cell-structure and its subcellular compartmentation via a cuticular organelle. However, several differences were observed between Eleodes and the two Tribolium species concerning the type 1 cells. First of all, T. castaneum type 1 cells do not form a mosaic of closely packed cells but appear singly or sometimes in groups of two cells attached to the reservoir. Second, cell-type 1 cuticular organelle was found to be a twisted canal inside cell 1 vesicle with a slight constriction defining the organelle's head, which is in agreement with the descriptions in Roth (1943) for T. confusum, but different from E. longicollis cell 1 cuticular organelle depicted in Eisner et al. (1964). It is therefore concluded that the more complex structure of type 1 cuticular organelle shown in Happ (1968) rather reflects the situation in $E$. longicollis than in T. castaneum. Moreover, in contrast to E. longicollis (Happ, 1968), neither T. castaneum (this study) nor T. confusum (Roth, 1943) were found to possess an extra tubulecarrying cell attached to type 1 cell. It should be noted, however, that in the work of Eisner et al. (1964) the authors were only vaguely speculating about the existence of such an accompanying cell in E. longicollis.

In agreement with the findings for $T$. confusum by Roth (1943), T. castaneum possesses an additional pair of defensive glands in its prothorax. These glands lack cell-type 1 but still produce the same main five volatiles with a similar molar ratio of quinones to alkenes (Li et al., 2013), as the abdominal glands do with their two types of gland cells. Together with the fact that in abdominal glands the type 2 cells are considered to be the cells synthesizing the 


\section{DISCUSSION}

benzoquinones, whereas the type 1 cells are believed to produce the alkenes (Happ, 1968; Li, 2013), differences in the morphology of type 2 cells between abdominal and prothoracic glands seemed not unlikely, as the prothoracic type 2 cells obviously have to manage the production of all secretion components in just one cell-type. However, light microscopic analysis in this study revealed no obvious morphological differences between prothoracic and abdominal type 2 cells. Also, a first comparison of transmission electron microscopic (TEM) pictures of abdominal and prothoracic type 2 gland cells did not detect major ultrastructural differences (data not shown). Admittedly, it should be noted that most of the TEM data are preliminary and able to only give a first impression on the gland ultrastructure. Therefore, a more detailed study is strongly recommended to answer this question comprehensively.

To my knowledge, the only tenebrionid beetle whose defensive glands have also been studied via transmission electron microscopy is E. longicollis (Eisner et al., 1964). First results of TEManalyzed T. castaneum glands described in this study suggest a high similarity of abdominal gland cell-type 2 for Tribolium and Eleodes even on ultrastructural level. Differences were so far only observed for the appearance of vesicle $2 b$, but these were minor and may be due to a quite diverse appearance of the vesicle depending on the cutting plane that has been analyzed in each study. In future studies, this established technique will be of good use to: (I) shed light onto the controversially discussed nature of cell-type 1 cuticular organelle as well as the existence of a possible accompanying tubule-carrying cell; and (II) study possible differences in the fine structure of type 2 cells from Tribolium abdominal and prothoracic glands in respect to their different profile of requirement in terms of secretion production. In sum, the overall structure of abdominal glands from Eleodes and Tribolium are highly similar, reflecting their taxonomic membership to the subfamily of Tenebrioninae.

\subsection{Prothoracic glandular $2 b$ cells display gene expression pattern from cell- type 1 in abdominal glands}

As already pointed out in section 5.1, prothoracic glands are able to produce the same gland volatile compounds within just one gland cell-type, whereas two cell-types are used in abdominal glands to produce alkenes in one cell-type and benzoquinones in the other cell-type. 
DISCUSSION

Besides structural differences between abdominal and prothoracic type 2 cells, one would also assume a changed expression profile of certain genes. During expression localization studies of several identified genes related to stink gland biology (this study and Li, 2013), it was observed that genes that are expressed in cell-type $2 \mathrm{a}$ in the abdominal gland are always also expressed in the respective cell-type $2 \mathrm{a}$ in the prothoracic gland (e.g. Lac2, Tc_010251, Tcas-ql VTGI (GT39), Figure 25). On the contrary, genes that are exclusively expressed in cell-type 1 in abdominal glands always show expression only in cell-type $2 \mathrm{~b}$ in the prothoracic glands (e.g. Prdx3, Tcas-al P450 (GT12), Figure 25), suggesting that these cells have the gene expression pattern from abdominal cell-type 1 and are responsible for alkene production in the prothoracic gland.

\section{3 iBeetle as a platform for the identification of genes related to gland secretion production and maintenance}

In the first phase of a genome-wide RNAi screen, about 5300 genes of the $T$. castaneum genome had been knocked down, analyzed for developmental and morphological defects, and annotated in the iBeetle-Base (Schmitt-Engel et al., 2015; Dönitz et al., 2015). In this study, 57 genes were found by searching the iBeetle-Base for entries reporting altered stink gland morphology and secretion in the adult. In a subsequent rescreen, the phenotype of 32 of them could be confirmed.

\subsubsection{Non-reproducible phenotypes in the rescreen}

In the rescreen, using non-overlapping fragments, the stink gland phenotype of 25 genes could not be reproduced, even though larval injections and higher dsRNA concentrations were tried. However, 19 of those had been annotated with phenotypes rather difficult to identify, like 'secretion color darker' (12 genes), 'less secretion' (4 genes) and 'irregular reservoir size' (3 genes, Figure 12). Given the fact that gland secretions of wildtype beetles do show natural variance in color and filling levels to some degree, it is much likely that these not-reproduced genes have been originally annotated as false-positives in the iBeetle screen. However, finding the remaining six genes to be non-reproducible was unexpected, as their knockdown phenotypes were clearly defined and easy to detect ('empty/necrotic' ( 4 genes) and 'melanized 


\section{DISCUSSION}

gland content' ( 2 genes)) and therefore can hardly be counted as falsely annotated in this context. Rather, one should think of possible off-target effects (due to a wrong gene annotation). This can easily be tested by re-injecting dsRNA of the original iBeetle fragment and dsRNA that covers both, the iBeetle fragment and the non-overlapping fragment.

Quality and quantity of RNAi phenotypes can also depend on the respective injected strain. For the rescreen the wildtypic Tribolium strain San Bernadino was used, whereas the iBeetle larval screen was performed with female larvae that were derived from a cross between D17Xred and pearl strains (Schmitt-Engel et al., 2015). As pointed out by Kitzmann et al. (2013), different strains may possess a general difference in RNAi sensitivity and/or may display nucleotide sequence divergence for certain genes. However, rescreen injections with higher dsRNA concentrations $(3 \mu \mathrm{g} / \mu \mathrm{l})$ also failed to reproduce the phenotypes, indicating that a dosesdependent effect between strains is unlikely in this case. Changes in nucleotide sequences of the six genes in different strains weren't checked yet but should be considered.

Finally, one should note that the penetrance with which the RNAi gland phenotypes occurred in the iBeetle screen was low (20-30\%) at least for three of the six non-reproduced ones (SG11, SG32 and SG26) suggesting a rather weak effect of the respective gene products on stink gland biology.

\subsubsection{Rescreen results provided the basis for a more slender second screening phase}

For the rescreen of stink gland affecting genes identified in the first phase of iBeetle, some changes in the analysis procedure have been made. Instead of larvae, mid-pupae were injected, and glands were already examined ten days instead of 40 days after adult eclosion. By doing so, the analysis procedure could be shortened significantly compared to the first phase iBeetle schedule. Importantly, stink gland phenotypes that have been reported during the iBeetle larval screen and were not observed in the pupal rescreen, could also not be reproduced by injecting into larvae. Thus, pupal injections, which are easier in animal handling and less time consuming, would have resulted in the identification of the same phenotype-causing genes. According to these findings, the screening schedule of the second iBeetle screening phase was slenderized by canceling the larval screen and analyzing morphological defects in stink glands 20 days after pupal injection instead. 
DISCUSSION

\subsubsection{Ontology of iBeetle-identified genes}

32 iBeetle-identified genes with a confirmed phenotype in the rescreen were categorized by their (predicted) molecular function derived from D. melanogaster orthologs. Not unexpected for examining a metabolic process, most candidates were identified as enzymes, followed by channels and transporters. Surprisingly, two transcription factors, midline and onecut that are mostly related to neurogenesis in Drosophila (e.g. Buescher et al., 2006; Nguyen et al., 2000), were identified. Interestingly, two candidate genes (TC_000885, Tc_014520) lack any known conserved domain. Neither a Drosophila nor any other ortholog was found and thus no molecular function could be predicted. However, they cannot be considered as stink gland specific genes, since they are only little and not specifically expressed in the glands (Tc_000885: ca. 100 reads in glands compared to 157 reads in reference tissue, Tc_014520: ca. 500 reads compared to 1025 reads in reference tissue, appendix 7.2,Table 12). In sum, the iBeetle screen identified a large group of genes with different molecular functions that all contribute to proper stink gland functioning.

\subsection{4 iBeetle-identified novel genes involved in stink gland secretion production}

In the first phase of iBeetle, which included 4480 genes in the larval screening part, the iBeetle screen discovered 32 genes that are involved in stink gland biological processes, partly strongly influencing the gland secretion chemical composition. In a previous study, 77 genes had been picked as candidates based only on their stink gland-specific high differential expression (at least $64 x$ higher expressed in the glands compared to the mid-abdominal reference tissue), and 29 of those were found to strongly influence stink gland morphology and gland content (Li, 2013). However, 48 genes had no or only little effect on the glands, despite the fact that they were highly and gland-specifically expressed genes, demonstrating that a high differential expression profile alone is no guaranty for finding proper candidates. In contrast, iBeetle is a genome-wide unbiased screen with the ambition to systematically knockdown every gene in the $T$. castaneum genome, not considering levels of (differential) gene expression. By checking the differential expression profiles of the 32 iBeetle-identified genes afterwards, only five were found to be highly differentially expressed in gland tissue ( $4 x$ higher than in reference tissue), including four genes that play a role in secretion production, but also one gene, that only shows a 


\section{DISCUSSION}

morphological enlargement of the abdominal glands. Vice versa, one of the BQ-less genes with very strong influence on BQ-production, was not differentially expressed but showed the same expression level as in the reference tissue. And even more striking, genes that were strongly related to benzoquinone synthesis in the beetle were found to be even underrepresented in stink gland tissue, concluding that highly differentially expressed genes are not necessarily linked to an essential function and, vice versa, genes with no or low differential expression levels can matter (Figure 24). However, one should keep in mind that highly differentially expressed genes may have homologs, which are able to rescue any knockdown effect, and therefore their importance would only arise from respective double- or even multiple knockdowns.

Data reflecting the effectiveness of iBeetle in identifying genes that already have been discovered via their differential expression profile are under investigation and will be presented elsewhere after the end of the second screening phase. At present, it can be stated that Lac2 and $\operatorname{Prd} x 3$ - both genes with high differential expression in gland tissue - were not detected in the iBeetle screen. In case of Lac2 this was due to a high mortality rate before the gland analysis, which was scheduled 40 days after hatching of the imago. In contrast, Prdx3 injections led to analyzable adult beetles. However, the gland phenotype that was detected for Prdx3 knockdown in this study has been missed in the iBeetle screen. As the two enhancer trap screen-identified genes Tc_007045 and Tc_013894 as well as the 29 genes examined by Li (2013) were not included in the first iBeetle screening phase, no statement can be made about their recovery today.

Altogether, these data clearly demonstrate the utility and necessity of phenotypic genetic screens for entire studies in general and the power of iBeetle to identify novel genes involved in stink gland biology in particular. Complete data will be presented elsewhere, with respect to the genes analyzed during the second screening phase. 
DISCUSSION

\subsection{Genes influencing the chemical composition of stink gland secretion}

By analyzing abdominal gland secretions from RNAi knockdowns via gas chromatography-mass spectrometry, a subset of genes that influenced the volatile chemical secretion composition was identified. In total, 19 genes (14 genes from iBeetle, two genes from an enhancer trap screen, three genes based on transcriptome data) caused abnormal secretion composition upon knockdown, which included the complete loss of benzoquinones (BQ-less), strong reductions of benzoquinones, strong and milder reductions of both benzoquinones and alkenes, and in rare cases gender-specific reductions of benzoquinones (Tables $4+5)$. None of the analyzed genes caused an alkene-less phenotype alone. However, besides two known phenoloxidase-encoding genes (Lac2 and Tyr1), four so far uncharacterized genes - Tc_010251, Tc_015818, Tc_011812, and Tc_012328 - were identified with no or almost no benzoquinones but wildtypic alkene levels upon knockdown, indicating that their products play critical and specific roles in the biosynthesis of benzoquinones and, more general, that benzoquinone- and alkene production seem to work independently from each other.

\subsubsection{Four uncharacterized BQ-less genes and their possible molecular function} Tc_010251 - a member of the cytochrome P450 family. Insect P450 enzymes act as monooxygenases that are highly divers in structure and function, and hence are also called mixed function oxidases (Feyereisen, 1999). They are involved in many important metabolic processes, like the synthesis and degradation of steroid molting hormones and juvenile hormones as well as in pheromone metabolism (Bergé et al., 1998). Another important role is the detoxification of insecticides from a natural or chemical origin (Frank and Fogleman, 1992; Taylor and Feyereisen, 1996). The insect genome encodes approximately 100 different P450 genes and therefore all P450 superfamily members are named following a special numeral nomenclature that is based on sequence homologies (Bergé et al., 1998).

pBLAST analysis of Tc_010251, whose encoding gene is expressed in type 2a cells of stink glands (Figure 25) and causes BQ-less gland secretions upon knockdown (Table 4), is predicted to encode for Cyp6a2, where 'Cyp' is the prefix for cytochrome P450 superfamily and '6a2' represents the family " 6 ", the subfamily "a" and the individual gene " 2 ", respectively. The Drosophila homolog CypP6a2 (CG9438) has been characterized and is known to play a major 


\section{DISCUSSION}

role in the metabolism of organophosphorus and organochlorine insecticides (Dunkov et al., 1997; Wan et al., 2014). However, this function can hardly be related to stink gland secretion production in Tribolium. But, besides the typical P450 domain, another conserved domain was found, indicating that Tribolium Cyp6a2 may belong to a P450-derived glycosyltransferase activator family. Members of this family resemble cytochrome P450 homologs that lack a critical heme-binding Cys residue. They are encoded next to a glycosyltransferase gene and it is shown that these P450 homologs physically interact with the glycosyltransferase protein helping it to achieve high specificity (Moncrieffe et al., 2012). As it is suggested that benzoquinones in the stink gland secretion of Tribolium are produced from phenolic glucosides and given the fact that upon gene knockdown of Cyp6a2 the secretions appear colorless and BQ-less, Cyp6a2 might be involved in the metabolism of these precursors by supporting the glycosyltransferase that catalyzes the glycosylic transfer. For future studies, it will be important to identify the responsible glycosylating enzyme. Interestingly, the knockdown of another Tribolium P450 enzyme, expressed in type 1 cells (and in $2 b$ cells in the prothoracic glands), resulted in alkeneless stink gland secretions (Tcas-al P450 (GT12) in Li, 2013), indicating that this protein much likely belongs to a P450 family different from Cyp6a2 and generally underlining the functional diversity of P450 enzymes.

Tc_015818 - a copper transporting ATPase. Copper is needed as a cofactor for a variety of enzymes. At the same time it is highly toxic as it can generate free radicals, which is why copper uptake and -disposal is strictly regulated by special membrane-bound transporters (Zhou et al., 2003). Once inside the cell, copper is transported to the trans-Golgi, where copper-transporting P-type ATPases import the copper, which subsequently becomes incorporated in copperdependent enzymes (Sellami et al., 2012). In humans, two genes encode for ATP7A and ATP7B. Defects in either of these ATPases lead to Menkes' disease and Wilson's disease, respectively, resulting in abnormal copper distribution in the body and very low levels of functional copperdependent enzymes, leading to neurodegeneration, irreversible hepatic damage, and untreated to death (Ala et al., 2007; Kaler, 2011). These copper transporting ATPases were found to be well conserved between mammals and insects. In D. melanogaster a single homolog, DmAtp7, covers for human ATP7A and ATP7B. Norgate et al. (2006) showed that DmAtp7 is essential in 
embryogenesis, early larval development, and adult pigmentation and is probably required for copper uptake from the diet.

Knockdown of gene Tc_015818 caused BQ-less stink gland secretions in T. castaneum. BLAST analysis suggested Drosophila Atp7 as the closest fly homolog with an amino acid sequence identity of 56\%. Therefore, Tc_015818 was designated as Atp7. Interestingly, for Atp7 two transcripts are annotated in the iBeetle-Base, encoding two identical proteins, except for 17 consecutive additional amino acids near the $\mathrm{N}$-terminus in one of the two proteins (not shown). One might consider that these two isoforms may correspond to human ATP7A and ATP7B.

Tribolium Atp7 knockdown analyses indicate a role in the production of benzoquinones in the stink glands. Considering the function of human and Drosophila Atp7 in copper homeostasis, one can hypothesize that Tribolium Atp7 is involved in providing copper-dependent enzymes with their essential cofactor. Such copper-dependent enzymes are for instance phenoloxidases, two of which have also been examined in this study for their possible role in benzoquinone production and are discussed in section 5.5.

Tc_011812 - a Death-associated protein kinase (DAPK)-related protein. Tc_011812 knockdown leads to stink gland secretions lacking benzoquinones. PBLAST analysis determined DmDrak as its closest fly homolog, a serine/threonine kinase related to the death-associated protein kinase (DAPK) family. Accordingly, Tc_011812 was named Drak. Members of the DAPK family have been linked to several cell death-related signaling pathways and functions other than cell death have also been proposed (Bialik and Kimchi, 2006). Neubueser and Hipfner (2010) showed that Drak promotes proper morphogenesis of epithelial tissues during $D$. melanogaster development and concluded that this kinase is a new component of the signaling networks that regulate the actin cytoskeleton to shape epithelial tissues. If and how this function can influence the benzoquinone production in T. castaneum is not known so far. The morphology of the gland and the secreting cells seemed to be normal in Drak knockdown situations, thus not providing evidence for mis-shaped tissues due to the lack of Drak protein.

Tc_012328 - a member of the peroxiredoxin family. Peroxiredoxins are a family of antioxidant enzymes that protect cells from hydrogen- and organic-peroxides via their peroxidase activity. 


\section{DISCUSSION}

They are important players in regulating redox homeostasis of the cell and are involved in cell signaling. Additionally, some peroxiredoxins display chaperone and phospholipase activity (Sharapov et al., 2014). Peroxiredoxins are divided into three classes: typical 2-Cys, atypical 2Cys, and 1-Cys peroxiredoxins, based on the number of cysteinyl residues involved in the catalysis and the mechanism used for recycling the cysteine sulfenic acid back to a thiol (Wood et al., 2003). Many organisms possess several peroxiredoxins. However, there is no common nomenclature for the isoforms. In the literature, mostly Prx or Prdx in combination with Roman or Arabic numerals are used. In mammals six different peroxiredoxins (here named as PrxlPrxVI) have been identified. Prxl-PrxIV belong to the typical 2-Cys class, PrxV is a member of atypical 2-Cys class, and PrxVI is a 1-Cys peroxiredoxin. Also, six Drosophila homologs (Prx1-6) were found, though one peroxiredoxin phylogenetically clusters with Prxl and Prxll, whereas two Drosophila peroxiredoxins are homologous to PrxVI (Lee et al., 2009; Radyuk et al., 2001). In this study, seven $T$. castaneum peroxiredoxins were identified, six of them clustering with the six known Drosophila peroxiredoxins and one additional homolog of Prxl/II that might be a silent or pseudo gene, as it is not expressed in stink glands and abdominal tissue of the beetle (TC_007526, Figure 20). As genes with peroxidase activity are believed to be involved in benzoquinone biosynthesis and maintenance in the stink glands, all Tribolium peroxiredoxins (except for the non-expressed one) were analyzed by RNAi knockdown and subsequent GC-MS analysis of gland content. Tc_012328, homologous to Drosophila Prx3 and mammalian PrxIII and henceforth called Prdx3, seemed to be involved in the benzoquinone metabolism of the gland, as in Prdx3 deficient beetles the gland secretion lacked the benzoquinones. Based on signal peptide analysis, Tribolium Prdx3 is much likely located to the mitochondria (Appendix 7.3), like its mammal and fly homologs (Radyuk et al., 2001). Studies about Drosophila mitochondrial peroxiredoxins Prx3 and Prx5 state that they specifically protect thioredoxin/glutathione systems, are critical regulators of global thiol homeostasis and play an important role in agedependent oxidative stress resistance (Kayashima and Yamakawa-Kobayashi, 2012; Radyuk et al., 2010). However, how this molecular function and hence Prdx3 can be linked to BQ synthesis in Tribolium stink glands remains elusive. 
DISCUSSION

\subsection{Phenoloxidases in $T$. castaneum stink gland tissue}

\subsubsection{Novel function for Lac2}

Laccases are multicopper oxidases with a broad substrate range. They are present in bacteria, fungi, plants and insects. In recent years, insect laccases have been mostly studied for their role in cuticle sclerotization and pigmentation, but some reports also describe laccase-like enzymatic activity in other tissues (Dittmer and Kanost, 2010). In T. castaneum two different laccases, Lac1 and Lac2, have been identified (Arakane et al., 2005). The molecular role of Lac1 is still unclear. But reports on $M$. sexta and An. gambiae orthologs suggest a potential involvement in diet metabolism or metal ion homeostasis (Dittmer et al., 2004; Gorman et al., 2008). On the contrary, Lac2 has a clear role in cuticle tanning (sclerotization and pigmentation) as shown by RNAi knockdown experiments revealing that Lac2 is required for catalyzing the oxidation of endogenous catechols that serve as precursors for cuticle tanning agents (Arakane et al., 2005). Here, a novel function of Tribolium Lac2 is proposed. Based on in situ hybridization studies, Lac2 was found to be expressed in adult stink gland tissue in an age-dependent manner, with strongest signal directly after hatching and decreasing expression from day ten on (Figure 25 and data not shown). Lac2 transcripts could be detected in both abdominal and prothoracic glands, but only in cells $2 a$ - the gland cell-type that is hypothesized to produce the benzoquinone components of gland secretion (Happ, 1968). Immunohistochemical stainings and enzymatic activity assays on gland tissue localized the active Lac2 protein in different subcellular compartments, tracing the transport of the protein to its destination, which is a cuticular organelle that connects the cell with the gland reservoir via a tubule. Also, active Lac2 was detected in the gland secretions, supporting that the enzyme accumulates first in the cuticular organelle before it is released together with the produced secretions into the reservoir of the gland. RNAi-mediated knockdown of Lac2 led to colorless gland secretion compared to yellowish fluid in wildtype beetles. Moreover, GC-MS analysis of gland content volatiles revealed that Lac2 knockdown gland secretions lack the two quinonoid components 2-methyl1,4-benzoquinone and 2-ethyl-1,4-benzoquinone. Taken together, these data identified Lac2 as the protein required for proper benzoquinone biosynthesis in the defensive stink glands of the red flour beetle, with the biochemical potential to catalyze the final oxidation step of $p$ - 


\section{DISCUSSION}

diphenolic precursors to their corresponding $p$-benzoquinones in the cuticular organelle of secretory cells 2 , as has been suggested as part of a possible BQ synthesis pathway in the glands by Happ (1968).

\subsubsection{Further effects of Lac2 knockdown on beetle tissues}

Beetle cuticle tanning. In five day-old Lac2 deficient beetles the exoskeleton appeared rather soft and lighter in color than wildtypic beetles of similar age, indicating a delay in sclerotization and pigmentation. In addition, many individuals displayed malformed elytra. However, these abnormalities are minor compared to the findings of Arakane et al. (2005). In their Tribolium Lac2 RNAi experiments, injected prepupae (= stage between larva-pupa molt) were tanned neither in pupal stage nor in adult stage and died within seven days after adult eclosion. In the present study, dsRNA injections were performed at mid-pupal stage. At this point of development (3-4 day old pupae), a first impulse of Lac2 expression is already completed (Arakane et al., 2005), providing sufficient Lac2 protein to at least start the tanning process. The resulting milder cuticle phenotype together with the little longer life span enabled the examination of stink glands in this work. Noticeably, although the tanning process was relatively little affected, stink glands exhibited strong alterations and malfunctions in the same individual, suggesting a tissue-specific Lac2 expression. Indeed, it is known that gland secretion production in T. castaneum starts not before the cuticle tanning process is completed (Unruh et al., 1998). Benzoquinones have been shown to be toxic to Tribolium larvae, which naturally lack a protective cuticle (Mondal, 1990; Verheggen et al., 2007). Therefore it would make sense to express Lac2 in the stink gland tissue only when the adult cuticle has been formed. Moreover, considering that the defensive $p$-benzoquinones as well as the catecholamine $o$-quinones used for beetle cuticle tanning use tyrosine as precursor, Unruh et al. (1998) suggested that the pathway of benzoquinone production 'may be suppressed in favor of the catecholamine quinone pathway until cuticle tanning agents are no longer needed'. Another possible control mechanism has been suggested by Yatsu and Asano (2009). They found evidence that $B$. mori cuticular Lac2A is synthesized as an inactive proenzyme that becomes later activated by proteolytic cleavage at the $\mathrm{N}$-terminus of the protein. However, other groups clearly demonstrated that full-length recombinant Lac2A from M. sexta, T. castaneum, and An. 
gambiae are constitutively active enzymes and that the removal of the amino-terminal region of the protein is not associated with increased activity (Dittmer et al., 2009; Gorman et al., 2012). Thus, Tribolium Lac2 is rather no zymogen but expressed as an active enzyme in a time- and tissue-dependent manner. Its activity in stink gland tissue is likely regulated through an expression temporally adjusted to the cuticle tanning process that starts earlier and in which Lac2 activity is predicted to be regulated by the availability of substrate (Gorman et al., 2012).

Melanin-like capsules. Lac2 deficient beetles also display plenty of small dark brown balls alongside their central nervous system directly under the surface of the abdominal epidermis (Figure 18C). These structures remind one of melanized capsules known as a result of immune response to parasitoid invaders in insects (e.g. Mahmoud et al., 2011; Pech and Strand, 1996) and therefore may suggest a link between the innate immune response and the external chemical defense of the beetle (see also section 5.6.2).

\subsubsection{Different functions of Lac2 splicing variants}

As reported previously by Arakane et al. (2005), two isoforms of Lac2 exist as a result of alternative splicing and were named $L a c 2 A$ and $L a c 2 B$. A similar $L a c 2$ gene structure leading to two isoforms has been described for An. gambiae and the lepidopteran B. mori (reviewed by Dittmer and Kanost, 2010). Tribolium Lac2A and Lac2B encode proteins of 717 and 712 aa, respectively. The overall amino acid sequence identity between the two proteins amounts to about $92 \%$, whereas in the variable C-terminal region only $74 \%$ are similar (Arakane et al., 2005). Expression profiles based on RT-PCR analysis of RNA prepared from prepupal, pupal and adult stages of the beetle indicated that Lac2A transcripts are more abundant than $\operatorname{Lac} 2 B$ transcripts at almost all time points examined (Arakane et al., 2005). Similar findings have been reported for BmLac2A and BmLac2B peptides purified from pupal cuticles (Yatsu and Asano, 2009). In Tribolium stink gland tissue, transcriptome data based on RNA-seq (Li et al., 2013) indicate that the Lac2 transcript mostly present in stink gland cells is Lac2A (Table 6), which was also supported by in situ hybridization experiments, in which no signal could be detected for the transcript Lac2B (Figure 25). However, a knockdown of this underrepresented isoform of Lac2 in stink gland tissue resulted in darker gland secretions and partly strongly reduced benzoquinone 


\section{DISCUSSION}

levels (Figure 18D; Table 5). Although the knockdown effect of the more abundant variant Lac2A was clearly stronger, as secretions were colorless and completely lacked the benzoquinone components, obviously both isoforms are needed for proper secretion biosynthesis, with Lac2A oxidizing benzoquinone precursors and an unclear role for Lac2B. Similarly, Arakane et al. (2005) showed that the contribution of Lac2B to beetle cuticle tanning is rather small but nonetheless indispensable, suggesting a role in morphogenesis and tanning of cuticle-containing tissues other than the exoskeleton. Thus, $L a c 2 B$ is a minor expressed isoform of $L a c 2$ but nevertheless essential for physiological processes in the beetle.

\subsubsection{Possible role for Lac2B in gland secretion producing cells}

In Lac2B deficient stink glands, melanin-like chemicals were found to accumulate in the reservoir. As DOPA is a precursor for the formation of melanin and at least present in epidermal cells of the beetle (Arakane et al., 2009), it is possible that Lac2B, displaying a protective function, somehow removes endogenous DOPA from the secretions, which otherwise would be further oxidized and would finally polymerize to melanin (Nappi and Christensen, 2005). One enzyme that is able to catalyze this reaction is PO, in the beetle known as Tyr1 (Arakane et al., 2005), which indeed is expressed in the gland tissue (Li et al., 2013 and this study) and causes altered secretions upon knockdown (Figure 18D). However, the contribution of DOPA and Tyr1 to stink gland secretion production is still elusive (see 5.6.2).

\subsubsection{ABTS is no suitable substrate for Lac2 in gland tissue}

ABTS has been reported many times as a typical artificial laccase substrate. For example, Hattori et al. (2005) described a laccase-dependent ABTS-oxidizing activity of salivary glands of the green rice leaf hopper, and Liang (2006) identified a Lac1 that oxidized ABTS in the salivary glands of the pea aphid. ABTS has also been used to study kinetic properties of recombinant Lac2 proteins from T. castaneum and An. gambiae (Gorman et al., 2012). Therefore, it was surprising that $A B T S$ was not processed by stink gland tissue. The oxidative reaction only occurred in the presence of hydrogen peroxide (Figure 28A). The resulting oxidized ABTS accumulated in gland cells that do not express Lac2 (Figure 31). Together with the fact that in an ABTS assay performed with purified recombinant Tribolium Lac2 enzyme, the presence of 
hydrogen peroxide even reduced the ABTS oxidation activity (Figure 29), these data strongly suggest that peroxidases rather than Lac2 catalyzed the oxidative reaction in the ABTS assay on gland tissue. The reason why Lac2 is active on ABTS in vitro as a recombinant protein but not in freshly dissected stink gland tissue remains unclear, the more so as it could be shown that the endogenous substrate L-DOPA was slowly but successfully oxidized to dopaquinone via Lac2 in the gland tissue (Figure 33), indicating that the enzyme itself is active. Interestingly, similar findings have been reported for phenol-oxidizing laccases from the termite gut (Coy et al., 2010). Here, native gut tissues as well as recombinant laccase proteins exhibited no activity towards ABTS, whereas L-DOPA was processed in both situations, though poorly compared to other phenolic substrates - a phenomenon that has been described for cuticular laccases in several studies (e.g. Andersen, 1978; Barrett, 1987; Sugumaran et al., 1992) and has been shown particularly for recombinant Tribolium Lac2 and suggested to be due to the high redox potential of L-DOPA compared to other phenolic substrates (Gorman et al., 2012).

\subsubsection{Tyr1 plays a (minor) role in stink gland benzoquinone production}

As a response to mechanical injuries and the invasion of microbial parasites, invertebrates produce melanin around the injured tissue or the respective intruder, the latter being encapsulated and thus prevented from further growth. The early steps in the pathway of hemolymphal melanin formation are catalyzed by the copper-containing enzyme PO. It is synthesized as an inactive proenzyme, referred to as prophenoloxidase (proPO), and gets activated by a limited proteolytic cleavage that is initiated by the proPO-activating system, which involves signaling molecules, serin proteinases and proPO inhibitor enzymes such as serpins (reviewed by Cerenius and Söderhäll, 2004; Cerenius et al., 2010).

In T. castaneum, two isoforms of PO have been found: Tyr1 and Tyr2. RNAi experiments from Arakane et al. (2005) revealed that neither of them is involved in the beetle cuticle tanning process. In the present study, it could be shown that RNAi-mediated knockdown of Tyr1 but not Tyr2 caused partially red gland secretions with strongly reduced levels of the benzoquinones (Figure 18D; Table 5), indicating a role for the melanin-producing enzyme Tyr1 also in benzoquinone gland secretion synthesis. A connection between the melanization pathway as part of the internal immune response and the benzoquinone production in $T$. castaneum has 


\section{DISCUSSION}

been suggested previously (Joop et al., 2014; Li et al., 2013). Li et al. (2013) identified three benzoquinone-less genes (Tcas-ql VTGI (GT39), Tcas-ql ARSB (GT62), Tcas-ql MRP (GT63)), whose knockdown led to reduced levels of general PO activity determined in a DOPA assay using homogenates of whole beetles. However, it should be noted that, as revealed in this study, in stink gland tissue DOPA oxidation is accomplished mainly by Lac2, whereas Tyr1 plays only a minor role. And as PO needs first a pathogen-induced activation, whereas Lac2 is constitutively active and expressed in epidermal and glandular cells, it seems likely that the measured PO activity in the experiment of Li et al. (2013) is mainly due to Lac2, suggesting that the authors rather showed the relation of the three benzoquinone-less genes to Lac2 than to the internal immune response. To clarify this issue, the PO activity assay from Li et al. (2013) should be repeated with activated Tyr1, e.g. by infecting the beetles with a pathogen some time before doing the PO activity measurements as has been done by Joop et al. (2014). Moreover, the identified BQ-less genes should also be tested for their involvement in DOPA oxidation in the gland tissue-specific DOPA assay that has been used in this study.

Nevertheless, RNAi knockdown results clearly demonstrate the involvement of Tyr1 in stink gland benzoquinone production. And the facts that melanin-like capsules appear obviously more frequently in Lac2 deficient beetles (Figure 18C), and that both pathways depend on the availability of tyrosine (Cerenius et al., 2008), strongly supports the idea of a metabolic linkage and mutual influence between the internal immune response, represented through $\mathrm{PO}$, and the external pathogen defense accomplished via the production of toxic benzoquinones by Lac2, as has been predicted previously by Joop et al. (2014).

\subsection{Model about benzoquinone production in Tribolium stink glands}

Based on several chemical and enzymatic tests on defensive secretion and gland tissue itself, Happ (1968) developed a model for benzoquinone production in stink gland tissue of $T$. castaneum that is still valid today. His model hypothesizes that the benzoquinone production takes place in cell-type $2 a$ and $2 b$, using a cuticle-lined vesicular organelle as a safe, sealed off reaction chamber to produce toxic benzoquinones. Enzymes that are predicted to work in the synthesis pathway are a glucosidase for precursor processing, a phenoloxidase to catalyze the 
final oxidation step of the phenolic precursor to benzoquinone and a peroxidase with an unclear role. In the present study, the phenoloxidase catalyzing the oxidation of $p$-diphenols to $p$ benzoquinones in Tribolium stink glands was found to be encoded by Lac2. Moreover, evidence was obtained for an involvement of the copper transporting ATPase Atp7 and the phenoloxidase responsible for wound healing and innate immune response Tyr1. The model in Figure 37 traces the secretory pathway of Lac2 - from its protein biosynthesis to its final destination. As shown via ISH, Lac2 is expressed only in cell 2a. The amino acid sequence of Lac2 contains an $\mathrm{N}$-terminal signal peptide, which targets Lac2 for co-translational translocation into the ER lumen. From here, packed in vesicles, the protein is proposed to be transported from the ER to the cis-Golgi, where it proceeds through the many compartments of the Golgi-apparatus until it reaches the trans-Golgi. Here, Atp7 is usually located and provides the copper ions, which are subsequently loaded onto Lac2 before it is thought to leave the Golgi in a secretory vesicle. This transport vesicle is hypothesized to fuse with the plasma membrane that lines the huge vacuole-like structure inside cell 2a (called vesicle), depositing Lac2 in its lumen. Then the protein can enter the cuticle-lined vesicular organelle at its porous "head" and is suggested to accumulate in the "bulb", where it oxidizes $p$-diphenols to their $p$-benzoquinones. Subsequently, the toxic products (as well as Lac2) run through a canal that connects the vesicular organelle with the reservoir of the gland, in which the chemicals are stored ready to use. Neither the localization of the other copper-dependent phenoloxidase Tyr1 in gland cells, nor its contribution to benzoquinone synthesis is clear yet, but due to its demonstrated influence on benzoquinone production one would expect it in a subcellular compartment of gland cell-type 2 . 
Cell 2a

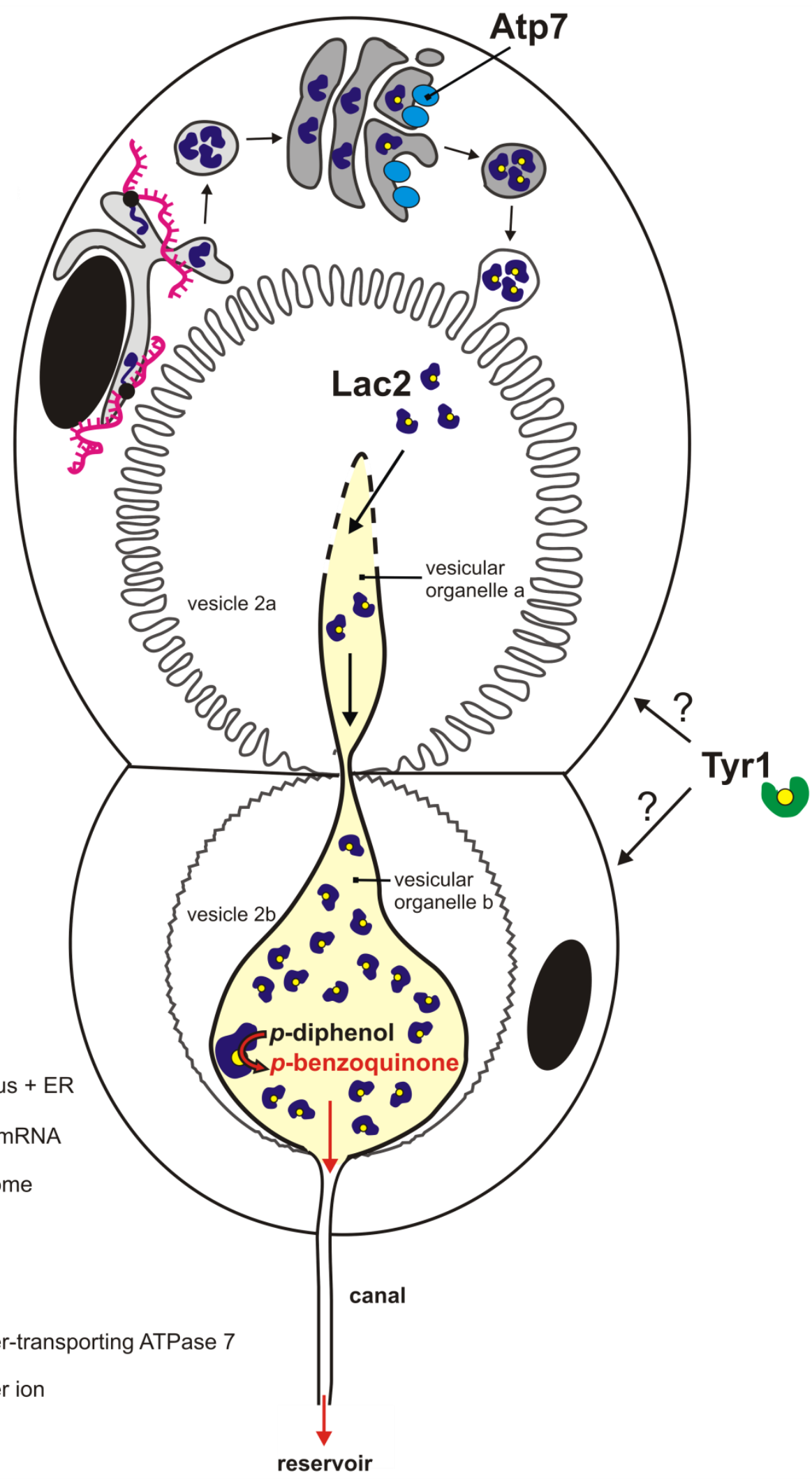

Figure 37: Model about the role of Lac2, Atp7, and Tyr1 in p-benzoquinone production in the stink glands of $T$. castaneum. For details see text. 
DISCUSSION

\subsection{Outlook}

With the identification of Lac2 as the phenoloxidase catalyzing the oxidation of $p$-diphenols to benzoquinones, one question mark can be removed from Happ's model (1968). However, genes that encode potentially involved glucosidases and peroxidases are still to be identified. In this study, four glucosidases that have been suggested as candidates by Li et al. (2013) based on their differential expression in stink glands were analyzed but found to have no influence on secretion production (data not shown). 13 further glucosidase encoding genes have been identified in Tribolium, most of them being underrepresented in gland tissue compared to tissue from the mid-abdomen of the beetle (Li et al., 2013). However, as it was shown in this study, even genes with comparably small numbers of transcript in stink glands can affect gland secretion production. Therefore, it will be worth to test the remaining glucosidases in future experiments. In addition, one should also look for glycosyltransferases and glucoside transporters to underline the proposed BQ-biosynthesis from phenolic glucoside precursors (Happ, 1968).

From the 28 Tribolium genes encoding proteins with predicted peroxidase activity, 11 genes were analyzed in this study, including four peroxidases that had been suggested by Li et al. (2013). In fact, one of those, Tc_012328 (Prdx3), was found to influence benzoquinone production, although its particular molecular role is elusive. Therefore, the remaining 17 genes should be checked in future studies, especially as this study showed that there is peroxidase activity in cell-type 1 , cell $2 \mathrm{~b}$ and in the efferent tubules of the beetle's stink gland (Figure 31 ). Li et al. (2013) and this study provided a large number of candidate genes for future stink gland studies. As mentioned above, there are much more genes to be discovered in the beetle's benzoquinone biosynthesis pathway. Furthermore, only little is known so far about the biosynthesis of the terminal olefins, which represent the second class of chemicals present in the gland secretion ( $\mathrm{Li}, 2013)$. From an evolutionary perspective, it will be interesting to investigate genes that contribute to abdominal but not to prothoracic gland secretion and vice versa. Moreover, several genes are present to study gender-specific differences. Due to the work of Bibi Atika (Department of Developmental Biology, Georg-August-University Göttingen), even more potential candidate genes involved in stink gland morphology and development are 


\section{DISCUSSION}

to come. She is currently analyzing candidate genes resulting from the second screening phase

of iBeetle and so far has identified several further genes with an impact on benzoquinone production. Interestingly, based on gene ontology analysis of those genes, it seems that the biosynthesis of benzoquinones is also linked to iron- and sulfur metabolism of the beetle. 
REFERENCES

\section{References}

Ala, A., Walker, A.P., Ashkan, K., Dooley, J.S., and Schilsky, M.L. (2007). Wilson's disease. Lancet Lond. Engl. 369, 397-408.

Alexander, P., and Barton, D.H.R. (1943). The excretion of ethylquinone by the flour beetle. Biochem. J. 37, 463-465.

Altschul, S.F., Gish, W., Miller, W., Myers, E.W., and Lipman, D.J. (1990). Basic local alignment search tool. J. Mol. Biol. 215, 403-410.

Andersen, S.O. (1978). Characterization of a trypsin-solubilized phenoloxidase from locust cuticle. Insect Biochem. 8, 143-148.

Andersen, S.O. (2010). Insect cuticular sclerotization: a review. Insect Biochem. Mol. Biol. 40, 166-178.

Aneshansley, D.J., Eisner, T., Widom, J.M., and Widom, B. (1969). Biochemistry at $100^{\circ} \mathrm{C}$ : Explosive Secretory Discharge of Bombardier Beetles (Brachinus). Science 165, 61-63.

Arakane, Y., Muthukrishnan, S., Beeman, R.W., Kanost, M.R., and Kramer, K.J. (2005). Laccase 2 is the phenoloxidase gene required for beetle cuticle tanning. Proc. Natl. Acad. Sci. U. S. A. 102, 11337-11342.

Arakane, Y., Lomakin, J., Beeman, R.W., Muthukrishnan, S., Gehrke, S.H., Kanost, M.R., and Kramer, K.J. (2009). Molecular and functional analyses of amino acid decarboxylases involved in cuticle tanning in Tribolium castaneum. J. Biol. Chem. 284, 16584-16594.

Arndt, E.M., Moore, W., Lee, W.-K., and Ortiz, C. (2015). Mechanistic origins of bombardier beetle (Brachinini) explosion-induced defensive spray pulsation. Science 348, 563-567.

Baldrian, P. (2006). Fungal laccases - occurrence and properties. FEMS Microbiol. Rev. 30, 215242.

Bannai, H., Tamada, Y., Maruyama, O., Nakai, K., and Miyano, S. (2002). Extensive feature detection of $\mathrm{N}$-terminal protein sorting signals. Bioinforma. Oxf. Engl. 18, 298-305.

Barrett, F.M. (1987). Phenoloxidases from larval cuticle of the sheep blowfly, Lucilia cuprina: Characterization, developmental changes, and inhibition by antiphenoloxidase antibodies. Arch. Insect Biochem. Physiol. 5, 99-118.

Beeman, R.W., Stuart, J.J., Haas, M.S., and Friesen, K.S. (1996). Chromosome extraction and revision of linkage group 2 in Tribolium castaneum. J. Hered. 87, 224-232. 


\section{REFERENCES}

Beheshti, N., and Mcintosh, A.C. (2007). The bombardier beetle and its use of a pressure relief valve system to deliver a periodic pulsed spray. Bioinspir. Biomim. 2, 57-64.

Bergé, J.B., Feyereisen, R., and Amichot, M. (1998). Cytochrome P450 monooxygenases and insecticide resistance in insects. Philos. Trans. R. Soc. B Biol. Sci. 353, 1701-1705.

Berghammer, A.J., Klingler, M., and Wimmer, E.A. (1999). A universal marker for transgenic insects. Nature 402, 370-371.

Binggeli, O., Neyen, C., Poidevin, M., and Lemaitre, B. (2014). Prophenoloxidase activation is required for survival to microbial infections in Drosophila. PLoS Pathog. 10, e1004067.

Blum, M.S. (1981). Chemical defenses of arthropods (New York: Academic Press Inc.).

Brown, S.J., Shippy, T.D., Miller, S., Bolognesi, R., Beeman, R.W., Lorenzen, M.D., Bucher, G., Wimmer, E.A., and Klingler, M. (2009). The red flour beetle, Tribolium castaneum (Coleoptera): a model for studies of development and pest biology. Cold Spring Harb. Protoc. 2009, pdb.emo126.

Bucher, G., Scholten, J., and Klingler, M. (2002). Parental RNAi in Tribolium (Coleoptera). Curr. Biol. 12, R85-R86.

Burmester, T. (2001). Molecular evolution of the arthropod hemocyanin superfamily. Mol. Biol. Evol. 18, 184-195.

Cerenius, L., and Söderhäll, K. (2004). The prophenoloxidase-activating system in invertebrates. Immunol. Rev. 116-126.

Cerenius, L., Lee, B.L., and Söderhäll, K. (2008). The proPO-system: pros and cons for its role in invertebrate immunity. Trends Immunol. 29, 263-271.

Cerenius, L., Kawabata, S., Lee, B.L., Nonaka, M., and Söderhäll, K. (2010). Proteolytic cascades and their involvement in invertebrate immunity. Trends Biochem. Sci. 35, 575-583.

Chapman, A.D. (2009). Numbers of living species in Australia and the world (Toowoomba, Australia).

Collins, P.J., Dobson, A.D.W., and Field, J.A. (1998). Reduction of the 2,2'-Azinobis(3Ethylbenzthiazoline-6-Sulfonate) Cation Radical by Physiological Organic Acids in the Absence and Presence of Manganese. Appl. Environ. Microbiol. 64, 2026-2031.

Consortium, T.U. (2015). UniProt: a hub for protein information. Nucleic Acids Res. 43, D204D212.

Corpet, F. (1988). Multiple sequence alignment with hierarchical clustering. Nucleic Acids Res. 16, 10881-10890. 
Coy, M.R., Salem, T.Z., Denton, J.S., Kovaleva, E.S., Liu, Z., Barber, D.S., Campbell, J.H., Davis, D.C., Buchman, G.W., Boucias, D.G., et al. (2010). Phenol-oxidizing laccases from the termite gut. Insect Biochem. Mol. Biol. 40, 723-732.

Crespo, R., Villaverde, M.L., Girotti, J.R., Güerci, A., Juárez, M.P., and de Bravo, M.G. (2011). Cytotoxic and genotoxic effects of defence secretion of Ulomoides dermestoides on A549 cells. J. Ethnopharmacol. 136, 204-209.

Cristino, A.S., Nunes, F.M.F., Barchuk, A.R., Aguiar-Coelho, V.M., Simões, Z.L.P., and Bitondi, M.M.G. (2010). Organization, evolution and transcriptional profile of hexamerin genes of the parasitic wasp Nasonia vitripennis (Hymenoptera: Pteromalidae). Insect Mol. Biol. 19 Suppl 1, 137-146.

Dettner, K., and Peters, W. (2003). Lehrbuch der Entymologie (Heidelberg - Berlin: Spektrum Akademischer Verlag).

Dittmer, N.T., and Kanost, M.R. (2010). Insect multicopper oxidases: diversity, properties, and physiological roles. Insect Biochem. Mol. Biol. 40, 179-188.

Dittmer, N.T., Suderman, R.J., Jiang, H., Zhu, Y.-C., Gorman, M.J., Kramer, K.J., and Kanost, M.R. (2004). Characterization of cDNAs encoding putative laccase-like multicopper oxidases and developmental expression in the tobacco hornworm, Manduca sexta, and the malaria mosquito, Anopheles gambiae. Insect Biochem. Mol. Biol. 34, 29-41.

Dittmer, N.T., Gorman, M.J., and Kanost, M.R. (2009). Characterization of endogenous and recombinant forms of laccase-2, a multicopper oxidase from the tobacco hornworm, Manduca sexta. Insect Biochem. Mol. Biol. 39, 596-606.

Dönitz, J., Schmitt-Engel, C., Grossmann, D., Gerischer, L., Tech, M., Schoppmeier, M., Klingler, M., and Bucher, G. (2015). iBeetle-Base: a database for RNAi phenotypes in the red flour beetle Tribolium castaneum. Nucleic Acids Res. 43, D720-D725.

Duehl, A.J., Arbogast, R.T., and Teal, P.E.A. (2011). Density-related volatile emissions and responses in the red flour beetle, Tribolium castaneum. J. Chem. Ecol. 37, 525-532.

Dunkov, B.C., Guzov, V.M., Mocelin, G., Shotkoski, F., Brun, A., Amichot, M., Ffrench-Constant, R.H., and Feyereisen, R. (1997). The Drosophila cytochrome P450 gene Cyp6a2: structure, localization, heterologous expression, and induction by phenobarbital. DNA Cell Biol. 16, 13451356.

Eichlerová, I., Šnajdr, J., and Baldrian, P. (2012). Laccase activity in soils: considerations for the measurement of enzyme activity. Chemosphere 88, 1154-1160.

Eisner, T., Meinwald, J., Monro, A., and Ghent, R. (1961). Defence mechanisms of arthropods-I The composition and function of the spray of the whipscorpion, Mastigoproctus giganteus (Lucas) (Arachnida, Pedipalpida). J. Insect Physiol. 6, 272-298. 


\section{REFERENCES}

Eisner, T., Mchenry, F., and Salpeter, M.M. (1964). Defense mechanisms of Arthropods. XV. Morphology of the quinone-producing glands of a tenebrionid beetle (Eleodes longicollis Lec.). J. Morphol. 115, 355-399.

Elias-Neto, M., Soares, M.P.M., Simões, Z.L.P., Hartfelder, K., and Bitondi, M.M.G. (2010). Developmental characterization, function and regulation of a Laccase2 encoding gene in the honey bee, Apis mellifera (Hymenoptera, Apinae). Insect Biochem. Mol. Biol. 40, 241-251.

Emanuelsson, O., Nielsen, H., Brunak, S., and Heijne, G. von (2000). Predicting subcellular localization of proteins based on their N-terminal amino acid sequence. J. Mol. Biol. 300, 10051016.

Emanuelsson, O., Brunak, S., Heijne, G. von, and Nielsen, H. (2007). Locating proteins in the cell using TargetP, SignalP and related tools. Nat. Protoc. 2, 953-971.

Endt, D.W. von, and Wheeler, J.W. (1971). 1-Pentadecene Production in Tribolium confusum. Science 172, 60-61.

Fawal, N., Li, Q., Savelli, B., Brette, M., Passaia, G., Fabre, M., Mathé, C., and Dunand, C. (2013). PeroxiBase: a database for large-scale evolutionary analysis of peroxidases. Nucleic Acids Res. 41, D441-D444.

Feyereisen, R. (1999). Insect P450 Enzymes. Annu. Rev. Entomol. 44, 507-533.

Frank, M.R., and Fogleman, J.C. (1992). Involvement of Cytochrome P450 in Host-Plant Utilization by Sonoran Desert Drosophila. Proc. Natl. Acad. Sci. U. S. A. 89, 11998-12002.

Futahashi, R., Tanaka, K., Matsuura, Y., Tanahashi, M., Kikuchi, Y., and Fukatsu, T. (2011). Laccase2 is required for cuticular pigmentation in stinkbugs. Insect Biochem. Mol. Biol. 41, 191196.

Gasteiger, E., Gattiker, A., Hoogland, C., Ivanyi, I., Appel, R.D., and Bairoch, A. (2003). ExPASy: the proteomics server for in-depth protein knowledge and analysis. Nucleic Acids Res. 31, 37843788.

González-Santoyo, I., and Córdoba-Aguilar, A. (2012). Phenoloxidase: a key component of the insect immune system. Entomol. Exp. Appl. 142, 1-16.

Görgen, G., Frößl, C., Boland, W., and Dettner, K. (1990). Biosynthesis of 1-alkenes in the defensive secretions ofTribolium confusum (Tenebrionidae); stereochemical implications. Experientia 46, 700-704.

Gorman, M.J., Dittmer, N.T., Marshall, J.L., and Kanost, M.R. (2008). Characterization of the multicopper oxidase gene family in Anopheles gambiae. Insect Biochem. Mol. Biol. 38, 817-824. 
Gorman, M.J., Sullivan, L.I., Nguyen, T.D.T., Dai, H., Arakane, Y., Dittmer, N.T., Syed, L.U., Li, J., Hua, D.H., and Kanost, M.R. (2012). Kinetic properties of alternatively spliced isoforms of laccase-2 from Tribolium castaneum and Anopheles gambiae. Insect Biochem. Mol. Biol. 42, 193-202.

Green, M.R., and Sambrook, J. (2012). Molecular Cloning: A Laboratory Manual (Fourth Edition): Three-Volume Set (Avon, Mass.: Cold Spring Harbor Laboratory).

Grill, C.P., and Moore, A.J. (1998). Effects of a larval antipredator response and larval diet on adult phenotype in an aposematic ladybird beetle. Oecologia 114, 274-282.

Happ, G.M. (1968). Quinone and hydrocarbon production in the defensive glands of Eleodes longicollis and Tribolium castaneum (Coleoptera, Tenebrionidae). J. Insect Physiol. 14, 18211837.

Hattori, M., Konishi, H., Tamura, Y., Konno, K., and Sogawa, K. (2005). Laccase-type phenoloxidase in salivary glands and watery saliva of the green rice leafhopper, Nephotettix cincticeps. J. Insect Physiol. 51, 1359-1365.

Hattori, M., Tsuchihara, K., Noda, H., Konishi, H., Tamura, Y., Shinoda, T., Nakamura, M., and Hasegawa, T. (2010). Molecular characterization and expression of laccase genes in the salivary glands of the green rice leafhopper, Nephotettix cincticeps (Hemiptera: Cicadellidae). Insect Biochem. Mol. Biol. 40, 331-338.

He, N., Botelho, J.M.C., McNall, R.J., Belozerov, V., Augustine Dunn, W., Mize, T., Orlando, R., and Willis, J.H. (2007). Proteomic analysis of cast cuticles from Anopheles gambiae by tandem mass spectrometry. Insect Biochem. Mol. Biol. 37, 135-146.

Hoegger, P.J., Kilaru, S., James, T.Y., Thacker, J.R., and Kües, U. (2006). Phylogenetic comparison and classification of laccase and related multicopper oxidase protein sequences. FEBS J. 273, 2308-2326.

Horn, T., and Boutros, M. (2010). E-RNAi: a web application for the multi-species design of RNAi reagents-2010 update. Nucleic Acids Res. 38, W332-W339.

Howard, R.W. (1987). Chemosystematic Studies of the Triboliini (Coleoptera: Tenebrionidae): Phylogenetic Inferences from the Defensive Chemicals of Eight Tribolium spp., Palorus ratzeburgi (Wissmann), and Latheticus oryzae Waterhouse. Ann. Entomol. Soc. Am. 80, 398405.

Joop, G., Roth, O., Schmid-Hempel, P., and Kurtz, J. (2014). Experimental evolution of external immune defences in the red flour beetle. J. Evol. Biol. 27, 1562-1571.

Kaler, S.G. (2011). ATP7A-related copper transport diseases-emerging concepts and future trends. Nat. Rev. Neurol. 7, 15-29. 


\section{REFERENCES}

Kayashima, Y., and Yamakawa-Kobayashi, K. (2012). Involvement of Prx3, a Drosophila ortholog of the thiol-dependent peroxidase PRDX3, in age-dependent oxidative stress resistance.

Biomed. Res. Tokyo Jpn. 33, 319-322.

Kearse, M., Moir, R., Wilson, A., Stones-Havas, S., Cheung, M., Sturrock, S., Buxton, S., Cooper, A., Markowitz, S., Duran, C., et al. (2012). Geneious Basic: an integrated and extendable desktop software platform for the organization and analysis of sequence data. Bioinforma. Oxf. Engl. 28, 1647-1649.

Keville, R., and Kannowski, P.B. (1975). Sexual excitation by pheromones of the confused flour beetle. J. Insect Physiol. 21, 81-84.

Kim, H.S., Murphy, T., Xia, J., Caragea, D., Park, Y., Beeman, R.W., Lorenzen, M.D., Butcher, S., Manak, J.R., and Brown, S.J. (2010). BeetleBase in 2010: revisions to provide comprehensive genomic information for Tribolium castaneum. Nucleic Acids Res. 38, D437-D442.

Kitzmann, P., Schwirz, J., Schmitt-Engel, C., and Bucher, G. (2013). RNAi phenotypes are influenced by the genetic background of the injected strain. BMC Genomics 14, 5.

Larkin, M.A., Blackshields, G., Brown, N.P., Chenna, R., McGettigan, P.A., McWilliam, H., Valentin, F., Wallace, I.M., Wilm, A., Lopez, R., et al. (2007). Clustal W and Clustal X version 2.0. Bioinforma. Oxf. Engl. 23, 2947-2948.

Laufer, Z., Beckett, R.P., and Minibayeva, F.V. (2006). Co-occurrence of the Multicopper Oxidases Tyrosinase and Laccase in Lichens in Sub-order Peltigerineae. Ann. Bot. 98, 1035-1042.

Lee, K.-S., lijima-Ando, K., lijima, K., Lee, W.-J., Lee, J.H., Yu, K., and Lee, D.-S. (2009). JNK/FOXOmediated neuronal expression of fly homologue of peroxiredoxin II reduces oxidative stress and extends life span. J. Biol. Chem. 284, 29454-29461.

Li, J. (2013). Fundamental Efforts to Develop Novel Biotechnological Approaches in Pest Management Applications against Coleoptera: Transcriptomic Exploration of the Chemical Defense Mechanism in the Red Flour Beetle, Tribolium castaneum.

Li, J., Lehmann, S., Weißbecker, B., Ojeda Naharros, I., Schütz, S., Joop, G., and Wimmer, E.A. (2013). Odoriferous Defensive stink gland transcriptome to identify novel genes necessary for quinone synthesis in the red flour beetle, Tribolium castaneum. PLoS Genet. 9, e1003596.

Liang, Q. (2006). Laccase-1 in the pea aphid, Acyrthosiphon pisum (Harris). Thesis. Kansas State University.

Loconti, J.D., and Roth, L.M. (1953). Composition of the Odorous Secretion of Tribolium Castaneum. Ann. Entomol. Soc. Am. 46, 281-289.

Lonergan, G., Mew, E., Schliephake, K., and Baker, W.L. (1997). Phenolic substrates for fluorometric detection of laccase activity. FEMS Microbiol. Lett. 153, 485-490. 
Lorenzen, M.D., Berghammer, A.J., Brown, S.J., Denell, R.E., Klingler, M., and Beeman, R.W. (2003). piggyBac-mediated germline transformation in the beetle Tribolium castaneum. Insect Mol. Biol. 12, 433-440.

Lorenzen, M.D., Kimzey, T., Shippy, T.D., Brown, S.J., Denell, R.E., and Beeman, R.W. (2007). piggyBac-based insertional mutagenesis in Tribolium castaneum using donor/helper hybrids. Insect Mol. Biol. 16, 265-275.

Lu, A., Zhang, Q., Zhang, J., Yang, B., Wu, K., Xie, W., Luan, Y.-X., and Ling, E. (2014). Insect prophenoloxidase: the view beyond immunity. Front. Physiol. 5.

Mahmoud, A.M.A., Luna-Santillana, E.J.D., and Rodríguez-Perez, M.A. (2011). Parasitism by the endoparasitoid, Cotesia flavipes induces cellular immunosuppression and enhances susceptibility of the sugar cane borer, Diatraea saccharalis to Bacillus thuringiensis. J. Insect Sci. $11,119$.

Marchler-Bauer, A., Derbyshire, M.K., Gonzales, N.R., Lu, S., Chitsaz, F., Geer, L.Y., Geer, R.C., He, J., Gwadz, M., Hurwitz, D.I., et al. (2015). CDD: NCBI's conserved domain database. Nucleic Acids Res. 43, D222-D226.

Markarian, H., Florentine, G.J., and Pratt Jr., J.J. (1978). Quinone production of some species of Tribolium. J. Insect Physiol. 24, 785-790.

Mason, H.S. (1947). The chemistry of melanin; the oxidation of di hydroxyphenylalanine by mammalian dopa oxidase. J. Biol. Chem. 168, 433-438.

McCaig, B.C., Meagher, R.B., and Dean, J.F.D. (2005). Gene structure and molecular analysis of the laccase-like multicopper oxidase (LMCO) gene family in Arabidopsis thaliana. Planta 221, 619-636.

Moncrieffe, M.C., Fernandez, M.-J., Spiteller, D., Matsumura, H., Gay, N.J., Luisi, B.F., and Leadlay, P.F. (2012). Structure of the glycosyltransferase EryCllI in complex with its activating P450 homologue EryCII. J. Mol. Biol. 415, 92-101.

Mondal, K.A.M.S.H. (1990). Effect of synthetic quinone and pheromone on Tribolium castaneum. Ann. Entomol. 8, 19-21.

Nappi, A.J., and Christensen, B.M. (2005). Melanogenesis and associated cytotoxic reactions: applications to insect innate immunity. Insect Biochem. Mol. Biol. 35, 443-459.

Neuberger, G., Maurer-Stroh, S., Eisenhaber, B., Hartig, A., and Eisenhaber, F. (2003a). Motif refinement of the peroxisomal targeting signal 1 and evaluation of taxon-specific differences. J. Mol. Biol. 328, 567-579. 


\section{REFERENCES}

Neuberger, G., Maurer-Stroh, S., Eisenhaber, B., Hartig, A., and Eisenhaber, F. (2003b). Prediction of peroxisomal targeting signal 1 containing proteins from amino acid sequence. J. Mol. Biol. 328, 581-592.

Neubueser, D., and Hipfner, D.R. (2010). Overlapping roles of Drosophila Drak and Rok kinases in epithelial tissue morphogenesis. Mol. Biol. Cell 21, 2869-2879.

Niu, B.-L., Shen, W.-F., Liu, Y., Weng, H.-B., He, L.-H., Mu, J.-J., Wu, Z.-L., Jiang, P., Tao, Y.-Z., and Meng, Z.-Q. (2008). Cloning and RNAi-mediated functional characterization of MaLac2 of the pine sawyer, Monochamus alternatus. Insect Mol. Biol. 17, 303-312.

Noh, M.Y., Beeman, R.W., and Arakane, Y. (2012). RNAi-based functional genomics in Tribolium castaneum and possible application for controlling insect pests. Entomol. Res. 42, 1-10.

Norgate, M., Lee, E., Southon, A., Farlow, A., Batterham, P., Camakaris, J., and Burke, R. (2006). Essential Roles in Development and Pigmentation for the Drosophila Copper Transporter DmATP7. Mol. Biol. Cell 17, 475-484.

Pappas, P.W., and Wardrop, S.M. (1996). Quantification of benzoquinones in the flour beetles. Tribolium castaneum and Tribolium confusum. Prep. Biochem. Biotechnol. 26, 53-66.

Parkinson, N., Smith, I., Weaver, R., and Edwards, J.P. (2001). A new form of arthropod phenoloxidase is abundant in venom of the parasitoid wasp Pimpla hypochondriaca. Insect Biochem. Mol. Biol. 31, 57-63.

Pech, L.L., and Strand, M.R. (1996). Granular cells are required for encapsulation of foreign targets by insect haemocytes. J. Cell Sci. 109, 2053-2060.

Pedrini, N., Ortiz-Urquiza, A., Huarte-Bonnet, C., Fan, Y., Juárez, M.P., and Keyhani, N.O. (2015). Tenebrionid secretions and a fungal benzoquinone oxidoreductase form competing components of an arms race between a host and pathogen. Proc. Natl. Acad. Sci. 201504552.

Peng, Z., Green, P.G., Arakane, Y., Kanost, M.R., and Gorman, M.J. (2014). A multicopper oxidase-related protein is essential for insect viability, longevity and ovary development. PloS One 9 , e111344.

Peschke, K., and Eisner, T. (1987). Defensive secretion of the tenebrionid beetle, Blaps mucronata: physical and chemical determinants of effectiveness. J. Comp. Physiol. [A] 161, 377388.

Prendeville, H.R., and Stevens, L. (2002). Microbe inhibition by Tribolium flour beetles varies with beetle species, strain, sex, and microbe group. J. Chem. Ecol. 28, 1183-1190.

Radyuk, S.N., Klichko, V.I., Spinola, B., Sohal, R.S., and Orr, W.C. (2001). The peroxiredoxin gene family in Drosophila melanogaster. Free Radic. Biol. Med. 31, 1090-1100. 
Radyuk, S.N., Rebrin, I., Klichko, V.I., Sohal, B.H., Michalak, K., Benes, J., Sohal, R.S., and Orr, W.C. (2010). Mitochondrial peroxiredoxins are critical for the maintenance of redox state and the survival of adult Drosophila. Free Radic. Biol. Med. 49, 1892-1902.

Reynolds, E.S. (1963). The use of lead citrate at high $\mathrm{pH}$ as an electron-opaque stain in electron microscopy. J. Cell Biol. 17, 208-212.

Richards, S., Gibbs, R.A., Weinstock, G.M., Brown, S.J., Denell, R., Beeman, R.W., Gibbs, R., Bucher, G., Friedrich, M., Grimmelikhuijzen, C.J.P., et al. (2008). The genome of the model beetle and pest Tribolium castaneum. Nature 452, 949-955.

Ronquist, F., and Huelsenbeck, J.P. (2003). MrBayes 3: Bayesian phylogenetic inference under mixed models. Bioinformatics 19, 1572-1574.

Ronquist, F., Huelsenbeck, J.P., and Teslenko, M. (2011). MrBayes version 3.2 Manual: Tutorials and Model Summaries.

Roth, L.M. (1943). Studies on the gaseous secretion of Tribolium confusum duval II. The odoriferous glands of Tribolium confusum. Ann. Entomol. Soc. Am. 36, 397-424.

Roth, L.M., and Howland, R.B. (1941). Studies on the Gaseous Secretion of Tribolium Confusum Duval I. Abnormalities Produced in Tribolium Confusum Duval by Exposure to a Secretion given off by the Adults. Ann. Entomol. Soc. Am. 34, 151-175.

Schierling, A., and Dettner, K. (2013). The pygidial defense gland system of the Steninae (Coleoptera, Staphylinidae): morphology, ultrastructure and evolution. Arthropod Struct. Dev. 42, 197-208.

Schildknecht, H., Holoubek, K., Weis, K.H., and Krämer, H. (1964). Defensive substances of the arthropods, their isolation and identification. Angew. Chemie Internat. 73-156.

Schinko, J.B., Weber, M., Viktorinova, I., Kiupakis, A., Averof, M., Klingler, M., Wimmer, E.A., and Bucher, G. (2010). Functionality of the GAL4/UAS system in Tribolium requires the use of endogenous core promoters. BMC Dev. Biol. 10, 53.

Schinko, J.B., Hillebrand, K., and Bucher, G. (2012). Heat shock-mediated misexpression of genes in the beetle Tribolium castaneum. Dev. Genes Evol. 222, 287-298.

Schmitt-Engel, C., Schultheis, D., Schwirz, J., Ströhlein, N., Troelenberg, N., Majumdar, U., Dao, V., Grossmann, D., Richter, T., Tech, M., et al. (2015). The iBeetle large-scale RNAi screen reveals gene functions for insect development and physiology. Nat Commun. 6

Sellami, A., Wegener, C., and Veenstra, J.A. (2012). Functional significance of the copper transporter ATP7 in peptidergic neurons and endocrine cells in Drosophila melanogaster. FEBS Lett. 586, 3633-3638. 


\section{REFERENCES}

Sharapov, M.G., Ravin, V.K., and Novoselov, V.I. (2014). Peroxiredoxins as multifunctional enzymes. Mol. Biol. 48, 520-545.

Sidjanski, S., Mathews, G.V., and Vanderberg, J.P. (1997). Electrophoretic separation and identification of phenoloxidases in hemolymph and midgut of adult Anopheles stephensi mosquitoes. J. Parasitol. 83, 686-691.

Sokoloff, A. (1972). The Biology of Tribolium - with special emphasis on genetic aspects (Oxford: Clarendon Press).

Sokoloff, A. (1974). The Biology of Tribolium - with special emphasis on genetic aspects (Oxford: Clarendon Press).

Solomon, E.I., Sundaram, U.M., and Machonkin, T.E. (1996). Multicopper Oxidases and Oxygenases. Chem. Rev. 96, 2563-2606.

Solomon, E.I., Augustine, A.J., and Yoon, J. (2008). O2 reduction to $\mathrm{H} 2 \mathrm{O}$ by the multicopper oxidases. Dalton Trans. Camb. Engl. 2003 3921-3932.

Sugumaran, M. (2002). Comparative biochemistry of eumelanogenesis and the protective roles of phenoloxidase and melanin in insects. Pigment Cell Res. Spons. Eur. Soc. Pigment Cell Res. Int. Pigment Cell Soc. 15, 2-9.

Sugumaran, M., Giglio, L., Kundzicz, H., Saul, S., and Semensi, V. (1992). Studies on the enzymes involved in puparial cuticle sclerotization in Drosophila melanogaster. Arch. Insect Biochem. Physiol. 19, 271-283.

Suzuki, T., Suzuki, T., Huynh, Y.M., and Muto, T. (1975). Hydrocarbon Repellents Isolated from Tribolium castaneum and T. confusum (Coleoptera: Tenebrionidae). Agric. Biol. Chem. 39, 22072211.

Taylor, M., and Feyereisen, R. (1996). Molecular biology and evolution of resistance to toxicants. Mol Biol Evol 719-734.

Terwilliger, N.B., and Ryan, M.C. (2006). Functional and phylogenetic analyses of phenoloxidases from brachyuran (Cancer magister) and branchiopod (Artemia franciscana, Triops longicaudatus) crustaceans. Biol. Bull. 210, 38-50.

Thomas, B.R., Yonekura, M., Morgan, T.D., Czapla, T.H., Hopkins, T.L., and Kramer, K.J. (1989). A trypsin-solubilized laccase from pharate pupal integument of the tobacco hornworm, Manduca sexta. Insect Biochem. 19, 611-622.

Trauner, J., Schinko, J., Lorenzen, M.D., Shippy, T.D., Wimmer, E.A., Beeman, R.W., Klingler, M., Bucher, G., and Brown, S.J. (2009). Large-scale insertional mutagenesis of a coleopteran stored grain pest, the red flour beetle Tribolium castaneum, identifies embryonic lethal mutations and enhancer traps. BMC Biol. 7, 73. 
Tschinkel, W.R. (1969). Phenols and quinones from the defensive secretions of the tenebrionid beetle, Zophobas rugipes. J. Insect Physiol. 15, 191-200.

Tschinkel, W.R. (1975a). A comparative study of the chemical defensive system of tenebrionid beetles: Chemistry of the secretions. J. Insect Physiol. 21, 753-783.

Tschinkel, W.R. (1975b). A comparative study of the chemical defensive system of tenebrionid beetles III. Morphology of the glands. J. Morphol. 145, 355-370.

Tschinkel, W.R. (1975c). A comparative study of the chemical defensive system of tenebrionid beetles: Chemistry of the secretions. J. Insect Physiol. 21, 753-783.

Tschinkel, W.R., and Doyen, J.T. (1980). Comparative anatomy of the defensive glands, ovipositors and female genital tubes of tenebrionid beetles (Coleoptera). Int. J. Insect Morphol. Embryol. 9, 321-368.

Unruh, L.M., Xu, R., and Kramer, K.J. (1998). Benzoquinone levels as a function of age and gender of the red flour beetle, Tribolium castaneum. Insect Biochem. Mol. Biol. 28, 969-977.

Verheggen, F., Ryne, C., Olsson, P.O.C., Arnaud, L., Lognay, G., Högberg, H.E., Persson, D., Haubruge, E., and Löfstedt, C. (2007). Electrophysiological and behavioral activity of secondary metabolites in the confused flour beetle, Tribolium confusum. J. Chem. Ecol. 33, 525-539.

Wan, H., Liu, Y., Li, M., Zhu, S., Li, X., Pittendrigh, B.R., and Qiu, X. (2014). Nrf2/Maf-binding-sitecontaining functional Cyp6a2 allele is associated with DDT resistance in Drosophila melanogaster. Pest Manag. Sci. 70, 1048-1058.

Wang, L., Wang, S., Li, Y., Paradesi, M.S.R., and Brown, S.J. (2007). BeetleBase: the model organism database for Tribolium castaneum. Nucleic Acids Res. 35, D476-D479.

Wirtz, R.A., Taylor, S.L., and Semey, H.G. (1978). Concentrations of substituted p-benzoquinones and 1-pentadecene in the red flour beetles Tribolium madens (Charp.) and T. brevicornis (Lec.) (Coleoptera, Tenebrionidae). Comp. Biochem. Physiol. 287-290.

Wood, Z.A., Schröder, E., Robin Harris, J., and Poole, L.B. (2003). Structure, mechanism and regulation of peroxiredoxins. Trends Biochem. Sci. 28, 32-40.

Xie, Z., Zhang, Y., Guliaev, A.B., Shen, H., Hang, B., Singer, B., and Wang, Z. (2005). The pbenzoquinone DNA adducts derived from benzene are highly mutagenic. DNA Repair 4, 13991409.

Yamazaki, H.I. (1972). Cuticular phenoloxidase from the silkworm, Bombyx mori: Properties, solubilization, and purification. Insect Biochem. 2, 431-444. 


\section{REFERENCES}

Yan, G., and Phillips, T.W. (1996). Influence of Tapeworm Infection on the Production of Aggregation Pheromone and Defensive Compounds in Tribolium castaneum. J. Parasitol. 82, 1037-1039.

Yatsu, J., and Asano, T. (2009). Cuticle laccase of the silkworm, Bombyx mori: purification, gene identification and presence of its inactive precursor in the cuticle. Insect Biochem. Mol. Biol. 39, 254-262.

Yezerski, A., Ciccone, C., Rozitski, J., and Volingavage, B. (2007). The effects of a naturally produced benzoquinone on microbes common to flour. J. Chem. Ecol. 33, 1217-1225.

Zhou, H., Cadigan, K.M., and Thiele, D.J. (2003). A copper-regulated transporter required for copper acquisition, pigmentation, and specific stages of development in Drosophila melanogaster. J. Biol. Chem. 278, 48210-48218. 


\section{Appendix}

\subsection{Oligonucleotides}

\begin{tabular}{|c|c|c|}
\hline Labeling & 5' --> 3' sequence & Application \\
\hline Tc_000223_F1 & CGAAGGCAAGAAGGTGAAAC & \multirow{2}{*}{ dsRNA + RNA probe } \\
\hline Tc_000223_R1 & ATTTTGATGCGATCGAGGTC & \\
\hline Tc_000223_3'RACE & GAGGGGCCAACGGCGTTGGAAGTC & \multirow{2}{*}{ RACE } \\
\hline Tc_000223_5'RACE & CAGCCGGTGCCCCCCGTGAG & \\
\hline Tc_000223_orf_for & ATGCTCCGTTCACTAGTGTTC & \multirow{2}{*}{ orf cloning } \\
\hline Tc_000223_orf_rev & CTACAACCTTATATTCACAGTGGGTAG & \\
\hline Tc_000240_F1 & AGGTGTGACAACAATGCTCAA & \multirow{2}{*}{ dsRNA } \\
\hline Tc_000240_R1 & AGACGACGTTATGAAGCGGT & \\
\hline Tc_000316_F1 & GTTTAGGCCGCCAGTTCAG & \multirow{2}{*}{ dsRNA } \\
\hline Tc_000316_R1 & GGGGTCCTGTGGTAAGGATT & \\
\hline Tc_000316_F2 & AGCCACTGGTCAACGGAATA & \multirow{2}{*}{ dsRNA } \\
\hline Tc_000316_R2 & CTTCGGTCACCTCCACACTT & \\
\hline Tc_000325_F1 & GCCACGAAAACTTCTCCCT & \multirow{2}{*}{ dsRNA } \\
\hline Tc_000325_R1 & TCAGCTTCACTACTCGTTTTAGGC & \\
\hline Tc_000379_F1 & CCACATTGGTGAGACGGAG & \multirow{2}{*}{ dsRNA } \\
\hline Tc_000379_R1 & ATGCGAGGTGAACGAGATTT & \\
\hline Tc_000379_F2 & CCCCGGGATCGGCATGCATG & \multirow{2}{*}{ dsRNA } \\
\hline Tc_000379_R2 & TGCCACGCCCCCATTGCTAA & \\
\hline Tc_000504_F1 & ACCGTGGCCACTGCCCTACA & \multirow{2}{*}{ dsRNA } \\
\hline Tc_000504_R1 & GAGCTGAGGCCACCATCGGC & \\
\hline Tc_000504_F2 & TGGACAATTGGTTGGACTCA & \multirow{2}{*}{ dsRNA } \\
\hline Tc_000504_R2 & CGCATTTTGGATGTGTTCCT & \\
\hline Tc_000885_3'RACE & CCAAGTACCAAGCCAGACGGGTCGGTGG & \multirow{2}{*}{ RACE } \\
\hline Tc_000885_5'RACE & TACTTGGCTGCCTGCTGGGGCCCCT & \\
\hline Tc_000885_F1 & ACCAAGCCAGACGGGTCGGT & \multirow{2}{*}{ dsRNA } \\
\hline Tc_000885_R1 & TTGCACCTCGACGGTGACGC & \\
\hline Tc_000885_F2 & CGAGTTCGTGGGAGCAGTAT & \multirow{2}{*}{ dsRNA + RNA probe } \\
\hline Tc_000885_R2 & AAAGCTTGTTTTCACGAAGCA & \\
\hline Tc_000885_F3 & GAGGGTCTCGGAGCAGTG & \multirow{2}{*}{ dsRNA } \\
\hline Tc_000885_R3 & GGCGGACTCTTGTTCGAT & \\
\hline Tc_002550_F1 & GCGCCAGCTACCAGATTTC & \multirow{2}{*}{ dsRNA } \\
\hline Tc_002550_R1 & CGGCAGTAGGGACACCTTT & \\
\hline Tc_002550_F3 & GTGGTCGGATATGCTGGTTC & \multirow{2}{*}{ dsRNA } \\
\hline Tc_002550_R3 & TGCAGTGCTTGTGGTAGAGG & \\
\hline Tc_002616_F1 & ACGTACCCAGCAACGGCCGA & \multirow{2}{*}{ dsRNA } \\
\hline Tc_002616_R1 & AGGACTCCACAGCCGCCTTTGA & \\
\hline Tc_002616_F2 & GATGGGTTTGCTGGGCTAT & \multirow{2}{*}{ dsRNA } \\
\hline Tc_002616_R2 & CAGTTCGCACAGCATATTCG & \\
\hline Tc_002723_F1 & GCCTCGTCTCCTTCACCC & \multirow{2}{*}{ dsRNA } \\
\hline Tc_002723_R1 & GTTGGTTGACGGGGATTAAC & \\
\hline
\end{tabular}




\section{APPENDIX}

\begin{tabular}{|c|c|c|}
\hline Tc_003063_F1 & ACTGGTGTGCTACAGGCCAA & \multirow{2}{*}{ dsRNA } \\
\hline Tc_003063_R1 & AGCTTCTCCAAAATGCCCTT & \\
\hline Tc_003409_F1 & TGAACCAGCTGTGCAAGGTC & \multirow{2}{*}{ dsRNA } \\
\hline Tc_003409_R1 & CTTTGCAAGCTGTCCGATTT & \\
\hline Tc_003409_F2 & CGCTCCAACTGGTTCAAGAT & \multirow{2}{*}{ dsRNA } \\
\hline Tc_003409_R2 & CTGTCCGACTCCGAGCTCTT & \\
\hline Tc_003857_F1 & CATAAGGCCCCTAATGAGCA & \multirow{2}{*}{ dsRNA } \\
\hline Tc_003857_R1 & AACACTTTATACGGCAGTTCGG & \\
\hline Tc_004126_F1 & TTCGAAAGCGAATTTGAAAC & \multirow{2}{*}{ dsRNA } \\
\hline Tc_004126_R1 & GCGCTTCTCTTCCTGCTCT & \\
\hline Tc_004129_F1 & AGTGTACCTGCCATCGCC & \multirow{2}{*}{ dsRNA } \\
\hline Tc_004129_R1 & AGGGCGGACATTTCTTTGAA & \\
\hline Tc_004129_F2 & ATGGACCACATAGGCGACA & \multirow{2}{*}{ dsRNA } \\
\hline Tc_004129_R2 & GGCGTCTTGTAAAACAGGAAG & \\
\hline Tc_004533_F1 & TGCCTTTGGACGAAAGTAAA & \multirow{2}{*}{ dsRNA } \\
\hline Tc_004533_R1 & AGTCACGGGTCAACTCCACT & \\
\hline Tc_004698_F1 & GAGACACCAAAGACAAGCCC & \multirow{2}{*}{ dsRNA } \\
\hline Tc_004698_R1 & GAGCGTCGTTGCTAGATCCT & \\
\hline Tc_004948_F1 & AAAAGAGGGCGCTCACAGT & \multirow{2}{*}{ dsRNA } \\
\hline Tc_004948_R1 & ATTGATAATTCGTGGTAGTGCG & \\
\hline Tc_005167_F1 & AGCTTTCCACCGAAAATGG & \multirow{2}{*}{ dsRNA } \\
\hline Tc_005167_R1 & GGAGGCACGGGAATAGATAG & \\
\hline Tc_005167_F2 & GCCAAAGCCGAGAAACGTA & \multirow{2}{*}{ dsRNA } \\
\hline Tc_005167_R2 & GCGCAAAGACATCGATCTAA & \\
\hline Tc_006098_F1 & CTCCAGAACCCGAAACTCC & \multirow{2}{*}{ dsRNA } \\
\hline Tc_006098_R1 & AAACTTCGTACAATCCGTTTCG & \\
\hline Tc_006098_F2 & ACTACAAGTTTGTGATTGGCCTG & \multirow{2}{*}{ dsRNA } \\
\hline Tc_006098_R2 & GCCATGGACACTCTGGATCT & \\
\hline Tc_006408_F1 & GGCGAAGAACGCCTGATAATG & \multirow{2}{*}{ dsRNA } \\
\hline Tc_006408_R1 & TCTCCAACTTTGGGGACAAC & \\
\hline Tc_006408_F2 & AGCAACAACACCAAGTTCGG & \multirow{2}{*}{ dsRNA } \\
\hline Tc_006408_R2 & CGTTATTGAAGTTCTTGCCG & \\
\hline Tc_006423_F1 & GTGCGTTTGTCAACGTCTCA & \multirow{2}{*}{ dsRNA } \\
\hline Tc_006423_R1 & TTTTCCACGATTTCTTTGCTG & \\
\hline Tc_006735_F1 & CTTTCGAACGGAGGAATCT & \multirow{2}{*}{ dsRNA } \\
\hline Tc_006735_R1 & CCATTTCTGGCCGTGTAAAG & \\
\hline Tc_007045_F1 & GCTATCTTAAGCGACAGGCG & \multirow{2}{*}{ dsRNA } \\
\hline Tc_007045_R1 & GGCGAGATTCTCCTTCAGATC & \\
\hline Tc_007650_F1 & TGCGGCATTTTGACCCTAAT & \multirow{2}{*}{ dsRNA } \\
\hline Tc_007650_R1 & CGCGTCAAAAATCTCCGTTA & \\
\hline Tc_008186_F1 & CTCCTGCATTTACCCCAATC & \multirow{2}{*}{ dsRNA } \\
\hline Tc_008186_R1 & GGGAGAATGCCTTTCCAGA & \\
\hline Tc_008608_F1 & GCTTGGAGACGGCGTTACT & \multirow{2}{*}{ dsRNA } \\
\hline Tc_008608_R1 & CATGTGCTCCTGGTTGTGTC & \\
\hline Tc_008608_F2 & GAAGAGACCGCCACAGGTC & \multirow{2}{*}{ dsRNA } \\
\hline Tc_008608_R2 & GGTTCTCCTGGCAAGGATTC & \\
\hline
\end{tabular}




\begin{tabular}{|c|c|c|}
\hline Tc_008936_F1 & CAAATCGGCGACTTCCTC & \multirow{2}{*}{ dsRNA } \\
\hline Tc_008936_R1 & CTTTCAACTTCGAACTCGTCG & \\
\hline Tc_008936_F2 & GAACGCGTTTATGGTGTGGT & \multirow{2}{*}{ dsRNA } \\
\hline Tc_008936_R2 & CCGGTTTCGTCGTCTTTTT & \\
\hline Tc_010033_F1 & GTGTCCTAGTCGGCGAAGGT & \multirow{2}{*}{ dsRNA } \\
\hline Tc_010033_R1 & AAACTTGTTTGGGCACTTACG & \\
\hline Tc_010251_3'RACE & CCCCCAGGTCAAACTTTGAATCGCCGC & \multirow{2}{*}{ RACE } \\
\hline Tc_010251_5'RACE & GGCCCATTTGGCGCGACAATTTGGG & \\
\hline Tc_010251_F1 & CCTTCGAAAATATCCCCCAG & \multirow{2}{*}{ dsRNA + RNA probe } \\
\hline Tc_010251_R1 & CCCGCTTTGTTATGGGACTA & \\
\hline Tc_010251_orf_for & ATGAACATCCCAAGCCCCTA & \multirow{2}{*}{ orf cloning } \\
\hline Tc_010251_orf_rev & GCCTATTTCACAATTTTTTCCACTCTA & \\
\hline Tc_010449_F1 & GTGTCCACTGTTACTCAACCCA & \multirow{2}{*}{ dsRNA } \\
\hline Tc_010449_R1 & GCACAGTTGACTTGTCTCGC & \\
\hline Tc_010484_F1 & ATAAGGGCACGCAAATCAAG & \multirow{2}{*}{ dsRNA } \\
\hline Tc_010484_R1 & TACTCCTCCAAGGTCCTTTGTT & \\
\hline Tc_011075_F1 & TGATGATCTGCACCAGGTTG & \multirow{2}{*}{ dsRNA } \\
\hline Tc_011075_R1 & TGTGCGACAAGTTCAAGTCC & \\
\hline Tc_011075_F2 & CGGACGAAATCGCAGACT & \multirow{2}{*}{ dsRNA } \\
\hline Tc_011075_R2 & GAACACTGGTCGTGGCATC & \\
\hline Tc_011159_F1 & AAAAATCGCATCTGCCAAAC & \multirow{2}{*}{ dsRNA } \\
\hline Tc_011159_R1 & AAGACAATGGGGACGTTGAC & \\
\hline Tc_011255_F1 & ATCGATGAGCTGTATGCTGAAA & \multirow{2}{*}{ dsRNA } \\
\hline Tc_011255_R1 & CTCAACCCGTTGGAATCCT & \\
\hline Tc_011288_F1 & CAGACCTTGAACTGGAAGGTCT & \multirow{2}{*}{ dsRNA } \\
\hline Tc_011288_R1 & GCATGCCTATAAACGACGG & \\
\hline Tc_011371_F1 & GCCGTTTACGACACCTGG & \multirow{2}{*}{ dsRNA } \\
\hline Tc_011371_R1 & CTGCTCGGTTTTGACGAAG & \\
\hline Tc_011812_3'RACE & CAGTGTCTGGGCGAGGCGGAGGC & \multirow{2}{*}{ RACE } \\
\hline Tc_011812_5'RACE & GGCCCCAAACGGCGTATAGCCCGACA & \\
\hline Tc_011812_F1 & CCGTAACCAGGACCAGATGA & \multirow{2}{*}{ dsRNA + RNA probe } \\
\hline Tc_011812_R1 & ACGATGTTCCTTTCGTGGAG & \\
\hline Tc_011969_F1 & AGACACGGTGGTTGAGCC & \multirow{2}{*}{ dsRNA } \\
\hline Tc_011969_R1 & TGGATCTTTCTTAAATCGGCA & \\
\hline Tc_012328_3'RACE & CCGTTGCCCCCACTTTGTGCGCTC & \multirow{2}{*}{ RACE } \\
\hline Tc_012328_5'RACE & GCCCAAACCCCCTTCAGATCTTTTTGTGT & \\
\hline Tc_012328_F1 & ACACCCCGCTCCTGACTTTA & \multirow{2}{*}{ dsRNA + RNA probe } \\
\hline Tc_012328_R1 & TCCTTGAGGGTCAGGTTTGA & \\
\hline Tc_012328_F2 & TCCTTGAGGGTCAGGTTTGA & \multirow{2}{*}{ dsRNA + RNA probe } \\
\hline Tc_012328_R2 & TGTGTGCCCCACTGAATTGA & \\
\hline Tc_012328_orf_for & ATGGCTGGACTATTTAGCACAA & \multirow{2}{*}{ orf cloning } \\
\hline Tc_012328_orf_rev & TTACTTATTAGCGGCTTGGAAAT & \\
\hline Tc_012539_F1 & GTCCAATTGTCATGGGTTGC & \multirow{2}{*}{ dsRNA } \\
\hline Tc_012539_R1 & TGATACGTGGTGGCAGGTC & \\
\hline Tc_012539_F2 & CGCCAACGGGGTTATTAAG & \multirow{2}{*}{ dsRNA } \\
\hline Tc_012539_R2 & CAGGACTTCCATGCTGTAGTTG & \\
\hline
\end{tabular}




\section{APPENDIX}

\begin{tabular}{|c|c|c|}
\hline Tc_012610_F1 & CACTGCAGGGACTGGCAC & \multirow{2}{*}{ dsRNA } \\
\hline Tc_012610_R1 & CCTCTAGTTCGTTGGGCTTG & \\
\hline Tc_012610_F2 & CATCGATTTGCAACACAACA & \multirow{2}{*}{ dsRNA } \\
\hline Tc_012610_R2 & CCGAACCTGACATTAGTTGAGTC & \\
\hline Tc_012642_F1 & GGTTCTCCCGATTTTCGCTA & \multirow{2}{*}{ dsRNA } \\
\hline Tc_012642_R1 & TCAGAGCTATTTGCACAACCTG & \\
\hline Tc_012828_F1 & CCAATACCACCACССССТ & \multirow{2}{*}{ dsRNA } \\
\hline Tc_012828_R1 & GGCGGCGTAAATTGGATAAT & \\
\hline Tc_012834_F1 & TACGTTGCACAGTTTCGGAG & \multirow{2}{*}{ dsRNA } \\
\hline Tc_012834_R1 & ACAACATTTGTCCGTCAAACC & \\
\hline Tc_012834_F2 & GATGGGTCCAGGACAGGTCT & \multirow{2}{*}{ dsRNA } \\
\hline Tc_012834_R2 & AATTCGCAATACAACTCGCA & \\
\hline Tc_012857_F1 & GTACTTAACGGAATTGGCCC & \multirow{2}{*}{ dsRNA } \\
\hline Tc_012857_R1 & CGCTAGATGTGGTTGCTTTTT & \\
\hline Tc_013513_3'RACE & GTCAAGTGGCGGGAGCACTCGGGC & \multirow{3}{*}{ RACE } \\
\hline Tc_013513_3'RACE_2 & GGATCCACATCGTCAAGTGGCGG & \\
\hline Tc_013513_5'RACE & CGGAGTAGAGGCGGAGGGATGAGCGC & \\
\hline Tc_013513_F1 & TTGACAACAAACGCTATCGG & \multirow{2}{*}{ dsRNA } \\
\hline Tc_013513_R1 & TTTCGTTGTTGGTGAGTTTGA & \\
\hline Tc_013513_F2 & CGGATCCACATCGTCAAGT & \multirow{2}{*}{ RNA probe } \\
\hline Tc_013513_R2 & ATGGGTTCGAATCGATTTTC & \\
\hline Tc_013791_F1 & GACTGGCTTGGCGACTCTT & \multirow{2}{*}{ dsRNA } \\
\hline Tc_013791_R1 & GGAATCCCCTGCGAATCT & \\
\hline Tc_013892_F1 & GCCGTTTCAGCCTGTTTCTA & \multirow{2}{*}{ dsRNA } \\
\hline Tc_013892_R1 & CGTTGGAGGCATTTGTTGAT & \\
\hline Tc_013894_3'RACE & CCGCCAAATGGGCTATGGTGGCGC & \multirow{3}{*}{ RACE } \\
\hline Tc_013894_5'RACE & AGCGGGATTATCGACTCCCGCTTCGGA & \\
\hline Tc_013894_5'RACE_2 & AGCCGCAGCGACGATGCACAAAC & \\
\hline Tc_013894_F1 & CATGTGTTTATGCCCTGACG & \multirow{2}{*}{ dsRNA + RNA probe } \\
\hline Tc_013894_R1 & TTCTGGTTCCGCACTAGGTT & \\
\hline Tc_013894_orf_for & ATGGACGCTGATAGCAGCTAC & \multirow{2}{*}{ orf cloning } \\
\hline Tc_013894_orf_rev & TCAATTATTGGGACGGGAAG & \\
\hline Tc_014205_F1 & AGGACGAACTGACGCTCTTC & \multirow{2}{*}{ dsRNA } \\
\hline Tc_014205_R1 & CAAAAGCTCTAGCCTCAGCC & \\
\hline Tc_014494_F1 & CCCATCCGATCATTGAACC & \multirow{2}{*}{ dsRNA } \\
\hline Tc_014494_R1 & АACTTGCTCAAAACTTCCGC & \\
\hline Tc_014520_F1 & CGACGCACATGAGAAGGTTA & \multirow{2}{*}{ dsRNA } \\
\hline Tc_014520_R1 & CAATTTGCGAAAATCTTGGTG & \\
\hline Tc_014520_F2 & CTGCGGCGTTATGTCTTCTA & \multirow{2}{*}{ dsRNA } \\
\hline Tc_014520_R2 & AGGCCTTATGTAAAGCCCCTT & \\
\hline Tc_014537_F1 & GCCAAGACTCCACCGACTAC & \multirow{2}{*}{ dsRNA } \\
\hline Tc_014537_R1 & CGAATTGCTCTAACCATCTCG & \\
\hline Tc_014544_F1 & CCCAGATGGTGTCCGAGTA & \multirow{2}{*}{ dsRNA } \\
\hline Tc_014544_R1 & TCGACACACACAAGTGGAGG & \\
\hline Tc_014544_F2 & TTTTCATCGCGTGCAAATAC & \multirow{2}{*}{ dsRNA } \\
\hline Tc_014544_R2 & CGCAAGAAGTGAATTGACAAA & \\
\hline
\end{tabular}




\begin{tabular}{|c|c|c|}
\hline Tc_014870_F1 & CGCTCCGTGACAACCAAT & \multirow{2}{*}{ dsRNA } \\
\hline Tc_014870_R1 & TGGTTCGAGTTGGCTGGTAG & \\
\hline Tc_014870_F2 & AATGCAGAACATGCGTTCG & \multirow{2}{*}{ dsRNA } \\
\hline Tc_014870_R2 & CCAATAGAACCCATCAGCGT & \\
\hline Tc_014887_F1 & GCCAGTGCTGGATGTGGT & \multirow{2}{*}{ dsRNA } \\
\hline Tc_014887_R1 & GCCCTAGCGTGGTTTTGAT & \\
\hline Tc_014907_F1 & ACAGCGTCGGATTTGGAG & \multirow{2}{*}{ dsRNA } \\
\hline Tc_014907_R1 & TCCCTCAGTTTTTGGTTTGC & \\
\hline Tc_014967_F1 & TAGTTTATCGGGGATCAGGG & \multirow{2}{*}{ dsRNA } \\
\hline Tc_014967_R1 & GGCATAGTTGTGGTCCCAAT & \\
\hline Tc_015095_F1 & TGTGATCCAAGCAGGCATTA & \multirow{2}{*}{ dsRNA } \\
\hline Tc_015095_R1 & GATTATCCACGAGATGGCGA & \\
\hline Tc_015165_F1 & AAACACGCGCGCTACTTAGA & \multirow{2}{*}{ dsRNA } \\
\hline Tc_015165_R1 & AAGGAATTGTTATTTGGGGTGA & \\
\hline Tc_015203_3'RACE & CGGGGGCTGTAGCTGGGGCTGCTTCTGG & \multirow{2}{*}{ RACE } \\
\hline Tc_015203_5'RACE & CCCACGCCGCTGCGATAATTCCGGCC & \\
\hline Tc_015203_F1 & GTTATAGGCAAATGGCCCAA & \multirow{2}{*}{ dsRNA } \\
\hline Tc_015203_R1 & TTATTTGTCACATCCCACGC & \\
\hline Tc_015203_orf_Notl_for & GCGGCCGCATGGGGGATTCCCAGATT & \multirow{2}{*}{ orf cloning } \\
\hline Tc_015203_orf_Xbal_rev & TCTAGACTAGCTGTTTAAAACATCTG & \\
\hline Tc_015379_3'RACE & TGGCCTTGGCCGCCTCTGATCGG & \multirow{2}{*}{ RACE } \\
\hline Tc_015379_5'RACE & CCCCGGCAGGGGCTCCTTCCAAA & \\
\hline Tc_015379_F1 & AATGGACCGTTGTTCGATTT & \multirow{2}{*}{ dsRNA + RNA probe } \\
\hline Tc_015379_R1 & GTGCTCAAATGGGCGAAA & \\
\hline Tc_015379_F2 & CCTGGAGGCTCAATGAAAAG & \multirow{2}{*}{ dsRNA ( $2^{\text {nd }}$ fragment) } \\
\hline Tc_015379_R2 & GTCTCCGGGCTGAAGGTATT & \\
\hline Tc_015379_orf_for & ATGGATCCTAGCAACCATG & \multirow{2}{*}{ orf cloning } \\
\hline Tc_015379_orf_rev & TTACACTAAATACTTATACAACATG & \\
\hline Tc_015811_F1 & CCAAAGCCGACCAAGTGT & \multirow{2}{*}{ dsRNA } \\
\hline Tc_015811_R1 & TCGAGGGACGTTATATTGGC & \\
\hline Tc_015818_3'RACE & GCAAGCTGACGTAGGGATGGCGATCGC & \multirow{2}{*}{ RACE } \\
\hline Tc_015818_5'RACE & GGGTTTGACGGTGGTCAGGGGCGG & \\
\hline Tc_015818_F1 & CCCCACAGGCGACTACAC & \multirow{2}{*}{ dsRNA + RNA probe } \\
\hline Tc_015818_R1 & TGTCAGTTGGCGTGTGTCAT & \\
\hline Tc_015818_orf_for & ATGGAACTGAGTACAGTGCGCT & \multirow{2}{*}{ orf cloning } \\
\hline Tc_015818_orf_rev & TTAAGTCATAAGTGGCTCACGTACG & \\
\hline Tc_015880_F1 & TCAACGGAAAAGGATCAAGTG & \multirow{2}{*}{ dsRNA } \\
\hline Tc_015880_R1 & CGAGTCTTTCTCCTTTGGTCA & \\
\hline Tc_015993_F1 & CCAAAACTGCCCGTCTATTC & \multirow{2}{*}{ dsRNA } \\
\hline Tc_015993_R1 & GGGAAAGACAGCTTGGTTGA & \\
\hline Tc_016254_F1 & CCGGAACGACTTGATCGTAA & \multirow{2}{*}{ dsRNA } \\
\hline Tc_016254_R1 & AAATCCCCGGATAGAGAATC & \\
\hline Tc_016254_F2 & AGAGTGAAGAAATCGTCCCTGA & \multirow{2}{*}{ dsRNA } \\
\hline Tc_016254_R2 & GGACTTGCGTAAACCCTGAA & \\
\hline Tc_030051_F1 & GGAGTCCAGAAGGCGAATTG & \multirow{2}{*}{ dsRNA } \\
\hline Tc_030051_R1 & CCAAATTGGCCGAACTGATA & \\
\hline
\end{tabular}




\section{APPENDIX}

\begin{tabular}{|c|c|c|}
\hline Tc_030051_F2 & CCGACAACATCATCAAAGGA & \multirow{2}{*}{ dsRNA } \\
\hline Tc_030051_R2 & CATGGAAATGGCAGCGTAAG & \\
\hline Tc_Lac2A_F1 & AACTCGAATCCTAACCTCGTT & \multirow{2}{*}{ dsRNA + RNA probe } \\
\hline Tc_Lac2A_R1 & ATGCAAGCGCCATATTGTA & \\
\hline Tc_Lac2A_orf_for & ATGGACGGAACACAACGG & \multirow{2}{*}{ orf cloning } \\
\hline Tc_Lac2A_orf_rev & TTAAACGAGGTTAGGATTCGAGTTA & \\
\hline Tc_Lac2B_F1 & AGATCGCCTGACCAGAATGT & \multirow{2}{*}{ dsRNA + RNA probe } \\
\hline Tc_Lac2B_R1 & TGATATAGGTGGCAGATGGTTC & \\
\hline Tc_Lac2AB_F1 & ATGCACGAAGACGCTACTGA & \multirow{2}{*}{ dsRNA + RNA probe } \\
\hline Tc_Lac2AB_R1 & TATGCCACACTCCCCTAAGC & \\
\hline TC_Lac2A_3'RACE & GGCCGAATGGCGTCTGTAGACTGTAGTGT & 3' RACE of variant $A$ \\
\hline TC_Lac2B_3'RACE & GACGCAAGCTGATCTACCGCCCGTTCC & 3' RACE of variant $B$ \\
\hline TC_Lac2AB_5'RACE & GCATTGACAGTGGGACCAGACGGTGTTGG & 5' RACE of both variants \\
\hline iPCR\#5_F1 & GACGCATGATTATCTTTTACGTGAC & \multirow{2}{*}{$1^{\text {st }}$ round of iPCR } \\
\hline iPCR\#6_R1 & TGACACTTACCGCATTGACA & \\
\hline iPCR\#7_F2 & GCGATGACGAGCTTGTTGGTG & \multirow{2}{*}{$2^{\text {nd }}$ round of iPCR } \\
\hline iPCR\#8_R2 & TCCAAGCGGCGACTGAGATG & \\
\hline iPCR\#9_seq & CGCGCTATTTAGAAAGAGAGAG & Sequencing primer \\
\hline pJET1.2_+T7_R & $\begin{array}{l}\text { TAATACGACTCACTATAGGAAGAACATCG } \\
\text { ATTTTCCATGGCAG }\end{array}$ & \multirow{2}{*}{$\begin{array}{l}\text { Addition of T7 overhangs } \\
\text { for dsRNA and probe } \\
\text { synthesis }\end{array}$} \\
\hline pJET1.2_F_up_T7 & ACACTTGTGCCTGAACACCATATC & \\
\hline pJET1.2F & CGACTCACTATAGGGAGAGCGGC & \multirow{2}{*}{ colony PCR (pJET1.2 vector) } \\
\hline pJET1.2R & AAGAACATCGATTTTCCATGGCAG & \\
\hline M13F & GTAAAACGACGGCCAGTG & \multirow{2}{*}{ colony PCR (pCRII vector) } \\
\hline M13R & CAGGAAACAGCTATGAC & \\
\hline
\end{tabular}




\subsection{Read count tables}

Table 8: Read counts for Tribolium phenoloxidase genes, taken from Li (2013).

\begin{tabular}{|c|c|c|c|c|c|c|c|c|c|c|}
\hline \multirow[t]{2}{*}{ OGS \# } & \multicolumn{2}{|c|}{ male - thx } & \multicolumn{2}{|c|}{ female - thx } & \multicolumn{2}{|c|}{ male - abd } & \multicolumn{2}{|c|}{ female - abd } & \multicolumn{2}{|c|}{$\begin{array}{c}\text { reference (mid } \\
\text { abdomen) }\end{array}$} \\
\hline & reads & depth & reads & depth & reads & depth & reads & depth & reads & depth \\
\hline \begin{tabular}{|l|} 
Tc_000325 \\
\end{tabular} & 309 & 5,73 & 351 & 6,51 & 793 & 14,707 & 198 & 3,67 & 317 & 5,88 \\
\hline TC_000821 & 1898 & 34,44 & 2041 & 37,04 & 2173 & 39,434 & 1192 & 21,63 & 770 & 13,97 \\
\hline Tc_005374 & 107 & 1,95 & 114 & 2,07 & 126 & 2,293 & 61 & 1,11 & 160 & 2,91 \\
\hline Tc_005375 & 24 & 0,43 & 24 & 0,43 & 53 & 0,956 & 65 & 1,17 & 94 & 1,70 \\
\hline Tc_005376 & 4649 & 78,62 & 1283 & 21,70 & 12125 & 205,051 & 5991 & 101,32 & 2059 & 34,82 \\
\hline Tc_005377 & 627 & 10,31 & 307 & 5,05 & 2908 & 47,837 & 1486 & 24,45 & 1013 & 16,66 \\
\hline Tc_006515 & 1847 & 33,23 & 2021 & 36,36 & 498 & 8,960 & 683 & 12,29 & 1470 & 26,45 \\
\hline Tc_006769 & 3636 & 65,89 & 1954 & 35,41 & 6581 & 119,255 & 3136 & 56,83 & 1372 & 24,86 \\
\hline Tc_010489 & 85149 & 1502,16 & 105542 & 1861,93 & 139482 & 2460,685 & 35974 & 634,64 & 4697 & 82,86 \\
\hline TC_010490 & 183 & 8,75 & 197 & 9,42 & 206 & 9,847 & 55 & 2,63 & 19 & 0,91 \\
\hline Tc_014907 & 1137 & 21,06 & 1238 & 22,93 & 1594 & 29,519 & 406 & 7,52 & 913 & 16,91 \\
\hline Tc_015848 & 367 & 13,96 & 394 & 14,99 & 508 & 19,323 & 126 & 4,79 & 377 & 14,34 \\
\hline TC_015849 & 706 & 24,64 & 779 & 27,18 & 996 & 34,755 & 259 & 9,04 & 422 & 14,73 \\
\hline TC_015880 & 528 & 11,26 & 492 & 10,49 & 518 & 11,046 & 337 & 7,19 & 337 & 7,19 \\
\hline
\end{tabular}

Table 9: Fold changes for Tribolium phenoloxidase genes, taken from Li (2013).

\begin{tabular}{|c|c|c|c|c|}
\hline \multirow{2}{*}{ OGS \# } & \multicolumn{4}{|c|}{ log2 Fold Change (sample reads/reference reads) } \\
\hline & $m-$ thx & $\mathrm{fm}-\mathrm{thx}$ & m - abd & fm - abd \\
\hline Tc_000325 & $-0,04$ & 0,15 & 1,32 & $-0,68$ \\
\hline Tc_000821 & 1,30 & 1,41 & 1,50 & 0,63 \\
\hline Tc_005374 & $-0,58$ & $-0,49$ & $-0,34$ & $-1,39$ \\
\hline Tc_005375 & $-1,97$ & $-1,97$ & $-0,83$ & $-0,53$ \\
\hline Tc_005376 & 1,17 & $-0,68$ & 2,56 & 1,54 \\
\hline Tc_005377 & $-0,69$ & $-1,72$ & 1,52 & 0,55 \\
\hline Tc_006515 & 0,33 & 0,46 & $-1,56$ & $-1,11$ \\
\hline Tc_006769 & 1,41 & 0,51 & 2,26 & 1,19 \\
\hline Tc_010489 & 4,18 & 4,49 & 4,89 & 2,94 \\
\hline Tc_010490 & 3,27 & 3,37 & 3,44 & 1,53 \\
\hline Tc_014907 & 0,32 & 0,44 & 0,80 & $-1,17$ \\
\hline Tc_015848 & $-0,04$ & 0,06 & 0,43 & $-1,58$ \\
\hline Tc_015849 & 0,74 & 0,88 & 1,24 & $-0,70$ \\
\hline Tc_015880 & 0,65 & 0,55 & 0,62 & 0,00 \\
\hline
\end{tabular}




\section{APPENDIX}

Table 10: Read counts for Tribolium peroxidase genes, taken from Li (2013).

\begin{tabular}{|c|c|c|c|c|c|c|c|c|c|c|}
\hline \multirow[t]{2}{*}{ Gene Name } & \multicolumn{2}{|c|}{ male - thx } & \multicolumn{2}{|c|}{ female - thx } & \multicolumn{2}{|c|}{ male - abd } & \multicolumn{2}{|c|}{ female - abd } & \multicolumn{2}{|c|}{$\begin{array}{c}\text { reference (mid } \\
\text { abdomen) }\end{array}$} \\
\hline & reads & depth & reads & depth & reads & depth & reads & depth & reads & depth \\
\hline Tc_000083 & 47141 & 1188,69 & 50216 & 1266,23 & 61575 & 1552,65 & 19049 & 480,33 & 4436 & 111,86 \\
\hline Tc_000175 & 229 & 4,30 & 210 & 3,95 & 53 & 1,00 & 61 & 1,15 & 923 & 17,35 \\
\hline Tc_000751 & 0 & 0 & 0 & 0,00 & 22 & 0,38 & 6 & 0,10 & 8 & 0,14 \\
\hline Tc_001071 & 595 & 30,89 & 612 & 31,77 & 742 & 38,52 & 1275 & 66,19 & 690 & 35,82 \\
\hline Tc_001556 & 103 & 0,94 & 63 & 0,57 & 415 & 3,78 & 1064 & 9,70 & 1111 & 10,13 \\
\hline Tc_001700 & 4 & 0,26 & 0 & 0,00 & 15 & 0,96 & 2 & 0,13 & 2 & 0,13 \\
\hline Tc_002498 & 138 & 1,15 & 118 & 0,99 & 625 & 5,22 & 526 & 4,39 & 234 & 1,96 \\
\hline Tc_004551 & 5065 & 86,46 & 3402 & 58,08 & 4371 & 74,62 & 8455 & 144,34 & 4029 & 68,78 \\
\hline Tc_004579 & 22 & 0,36 & 12 & 0,20 & 32 & 0,52 & 88 & 1,44 & 213 & 3,49 \\
\hline Tc_004592 & 42 & 0,56 & 50 & 0,67 & 40 & 0,53 & 24 & 0,32 & 825 & 10,98 \\
\hline Tc_004593 & 118 & 3,68 & 161 & 5,02 & 190 & 5,93 & 75 & 2,34 & 508 & 15,85 \\
\hline Tc_004661 & 13 & 0,27 & 18 & 0,38 & 14 & 0,29 & 9 & 0,19 & 1716 & 35,99 \\
\hline Tc_004948 & 8837 & 499,71 & 3809 & 215,39 & 4290 & 242,59 & 1881 & 106,37 & 1800 & 101,79 \\
\hline Tc_005493 & 137 & 1,18 & 86 & 0,74 & 320 & 2,76 & 33740 & 291,32 & 932 & 8,05 \\
\hline Tc_007526 & 0 & 0 & 0 & 0 & 0 & 0 & 0 & 0 & 0 & 0 \\
\hline Tc_007642 & 1348 & 108,76 & 1285 & 103,67 & 1562 & 126,02 & 1734 & 139,90 & 1815 & 146,43 \\
\hline Tc_010354 & 206 & 13,11 & 169 & 10,76 & 234 & 14,89 & 153 & 9,74 & 245 & 15,59 \\
\hline Tc_010355 & 2036 & 152,60 & 2002 & 150,05 & 3375 & 252,96 & 1402 & 105,08 & 1207 & 90,47 \\
\hline Tc_010362 & 4673 & 295,96 & 4959 & 314,07 & 8300 & 525,67 & 3754 & 237,75 & 1544 & 97,79 \\
\hline Tc_011090 & 280 & 7,39 & 374 & 9,87 & 361 & 9,53 & 228 & 6,02 & 265 & 6,99 \\
\hline Tc_011222 & 84 & 1,18 & 20 & 0,28 & 39 & 0,55 & 419 & 5,87 & 525 & 7,36 \\
\hline Tc_011385 & 114 & 2,79 & 96 & 2,35 & 69 & 1,69 & 70 & 1,72 & 235 & 5,76 \\
\hline Tc_012328 & 8537 & 462,12 & 9570 & 518,03 & 8048 & 435,65 & 3967 & 214,74 & 1545 & 83,63 \\
\hline Tc_013282 & 1 & 0,07 & 0 & 0 & 0 & 0 & 0 & 0 & 18 & 1,23 \\
\hline Tc_013283 & 18 & 0,21 & 14 & 0,16 & 3 & 0,03 & 10 & 0,11 & 491 & 5,61 \\
\hline Tc_013791 & 304 & 17,50 & 228 & 13,13 & 190 & 10,94 & 143 & 8,23 & 157 & 9,04 \\
\hline Tc_014929 & 5337 & 343,16 & 5140 & 330,49 & 5492 & 353,12 & 4799 & 308,57 & 4080 & 262,34 \\
\hline Tc_015234 & 36 & 0,42 & 26 & 0,31 & 91 & 1,07 & 45 & 0,53 & 186 & 2,19 \\
\hline
\end{tabular}


Table 11: Fold changes for Tribolium peroxidase genes, taken from Li (2013).

\begin{tabular}{|c|c|c|c|c|}
\hline \multirow{2}{*}{ OGS \# } & \multicolumn{4}{|c|}{ log2 Fold Change (sample reads/reference reads) } \\
\hline & $\mathrm{m}$ - the & fm - thx & m-abd & fm - abd \\
\hline Tc_000083 & 3,41 & 3,50 & 3,80 & 2,10 \\
\hline Tc_000175 & $-2,01$ & $-2,14$ & $-4,12$ & $-3,92$ \\
\hline Tc_000751 & & & 1,46 & $-0,42$ \\
\hline Tc_001071 & $-0,21$ & $-0,17$ & 0,10 & 0,89 \\
\hline Tc_001556 & $-3,43$ & $-4,14$ & $-1,42$ & $-0,06$ \\
\hline Tc_001700 & 1,00 & & 2,91 & 0,00 \\
\hline Tc_002498 & $-0,76$ & $-0,99$ & 1,42 & 1,17 \\
\hline Tc_004551 & 0,33 & $-0,24$ & 0,12 & 1,07 \\
\hline Tc_004579 & $-3,28$ & $-4,15$ & $-2,73$ & $-1,28$ \\
\hline Tc_004592 & $-4,30$ & $-4,04$ & $-4,37$ & $-5,10$ \\
\hline Tc_004593 & $-2,11$ & $-1,66$ & $-1,42$ & $-2,76$ \\
\hline Tc_004661 & $-7,04$ & $-6,57$ & $-6,94$ & $-7,57$ \\
\hline Tc_004948 & 2,30 & 1,08 & 1,25 & 0,06 \\
\hline Tc_005493 & $-2,77$ & $-3,44$ & $-1,54$ & 5,18 \\
\hline \multicolumn{5}{|l|}{ Tc_007526 } \\
\hline Tc_007642 & $-0,43$ & $-0,50$ & $-0,22$ & $-0,07$ \\
\hline Tc_010354 & $-0,25$ & $-0,54$ & $-0,07$ & $-0,68$ \\
\hline Tc_010355 & 0,75 & 0,73 & 1,48 & 0,22 \\
\hline Tc_010362 & 1,60 & 1,68 & 2,43 & 1,28 \\
\hline Tc_011090 & 0,08 & 0,50 & 0,45 & $-0,22$ \\
\hline Tc_011222 & $-2,64$ & $-4,71$ & $-3,75$ & $-0,33$ \\
\hline Tc_011385 & $-1,04$ & $-1,29$ & $-1,77$ & $-1,75$ \\
\hline Tc_012328 & 2,47 & 2,63 & 2,38 & 1,36 \\
\hline Tc_013282 & $-4,17$ & & & \\
\hline Tc_013283 & $-4,77$ & $-5,13$ & $-7,35$ & $-5,62$ \\
\hline Tc_013791 & 0,95 & 0,54 & 0,28 & $-0,13$ \\
\hline Tc_014929 & 0,39 & 0,33 & 0,43 & 0,23 \\
\hline Tc_015234 & $-2,37$ & $-2,84$ & $-1,03$ & $-2,05$ \\
\hline
\end{tabular}




\section{APPENDIX}

Table 12: Read counts for 32 iBeetle-identified + 2 enhancer trap screen genes, taken from Li (2013).

\begin{tabular}{|c|c|c|c|c|c|c|c|c|c|c|}
\hline \multirow{2}{*}{$\begin{array}{l}\text { Gene } \\
\text { Name }\end{array}$} & \multicolumn{2}{|c|}{ male - thx } & \multicolumn{2}{|c|}{ female - th $x$} & \multicolumn{2}{|c|}{ male - abd } & \multicolumn{2}{|c|}{ female - abd } & \multicolumn{2}{|c|}{$\begin{array}{c}\text { reference (mid } \\
\text { abdomen) }\end{array}$} \\
\hline & reads & depth & reads & depth & reads & depth & reads & depth & reads & depth \\
\hline Tc_000240 & 16 & 0,40 & 22 & 0,55 & 16 & 0,40 & 36 & 0,90 & 279 & 6,97 \\
\hline Tc_000379 & 2466 & 143,28 & 2331 & 135,44 & 2332 & 135,50 & 3090 & 179,54 & 1031 & 59,91 \\
\hline Tc_000504 & 45 & 0,92 & 99 & 2,02 & 85 & 1,73 & 122 & 2,49 & 13 & 0,27 \\
\hline Tc_000885 & 144 & 5,85 & 113 & 4,59 & 54 & 2,19 & 10 & 0,41 & 157 & 6,37 \\
\hline Tc_002616 & 2227 & 58,77 & 2140 & 56,47 & 2323 & 61,30 & 3540 & 93,42 & 1747 & 46,10 \\
\hline Tc_002723 & 2823 & 12,35 & 2279 & 9,97 & 3947 & 17,27 & 6931 & 30,33 & 4815 & 21,07 \\
\hline Tc_003409 & 44 & 1,86 & 49 & 2,07 & 40 & 1,69 & 53 & 2,24 & 19 & 0,80 \\
\hline Tc_004129 & 8 & 0,18 & 6 & 0,13 & 8 & 0,18 & 0 & 0,00 & 41 & 0,92 \\
\hline TC_005167 & 670 & 9,19 & 672 & 9,22 & 1101 & 15,11 & 1069 & 14,67 & 1211 & 16,62 \\
\hline Tc_006408 & 7507 & 270,91 & 7545 & 272,28 & 8356 & 301,55 & 7917 & 285,70 & 14097 & 508,72 \\
\hline TC_008608 & 144 & 1,72 & 69 & 0,83 & 179 & 2,14 & 176 & 2,11 & 2111 & 25,25 \\
\hline Tc_010251 & 29710 & 760,26 & 33432 & 855,50 & 39562 & 1012,36 & 14185 & 362,98 & 1510 & 38,64 \\
\hline Tc_011075 & 153 & 10,77 & 105 & 7,39 & 150 & 10,56 & 625 & 43,98 & 3516 & 247,42 \\
\hline TC_011288 & 156 & 1,66 & 115 & 1,23 & 95 & 1,01 & 60 & 0,64 & 1637 & 17,44 \\
\hline Tc_011812 & 7031 & 261,94 & 7559 & 281,61 & 8061 & 300,31 & 2731 & 101,74 & 951 & 35,43 \\
\hline Tc_012539 & 245 & 1,60 & 190 & 1,24 & 280 & 1,82 & 403 & 2,63 & 984 & 6,41 \\
\hline Tc_012610 & 85 & 1,02 & 43 & 0,51 & 109 & 1,30 & 69 & 0,82 & 147 & 1,76 \\
\hline TC_012828 & 89 & 4,32 & 44 & 2,14 & 1020 & 49,50 & 433 & 21,01 & 927 & 44,99 \\
\hline TC_012834 & 449 & 4,54 & 401 & 4,05 & 672 & 6,79 & 486 & 4,91 & 931 & 9,40 \\
\hline Tc_013513 & 444 & 15,12 & 484 & 16,48 & 826 & 28,13 & 204 & 6,95 & 21 & 0,72 \\
\hline Tc_013892 & 601 & 25,55 & 502 & 21,34 & 683 & 29,03 & 1110 & 47,18 & 1139 & 48,41 \\
\hline Tc_014494 & 456 & 13,25 & 492 & 14,29 & 546 & 15,86 & 463 & 13,45 & 372 & 10,81 \\
\hline Tc_014520 & 556 & 11,40 & 499 & 10,23 & 576 & 11,81 & 521 & 10,68 & 1025 & 21,01 \\
\hline Tc_014870 & 1 & 0,01 & 2 & 0,03 & 9 & 0,13 & 0 & 0,00 & 77 & 1,14 \\
\hline Tc_014887 & 210 & 10,43 & 168 & 8,35 & 504 & 25,04 & 2334 & 115,94 & 437 & 21,71 \\
\hline Tc_015095 & 1527 & 39,15 & 1831 & 46,95 & 1367 & 35,05 & 1031 & 26,44 & 881 & 22,59 \\
\hline Tc_015165 & 550 & 30,42 & 515 & 28,49 & 557 & 30,81 & 425 & 23,51 & 241 & 13,33 \\
\hline Tc_015203 & 427 & 10,36 & 344 & 8,35 & 515 & 12,50 & 73 & 1,77 & 251 & 6,09 \\
\hline Tc_015379 & 616 & 16,57 & 487 & 13,10 & 2226 & 59,86 & 3212 & 86,38 & 132 & 3,55 \\
\hline Tc_015811 & 682 & 21,23 & 757 & 23,56 & 881 & 27,42 & 814 & 25,33 & 367 & 11,42 \\
\hline Tc_015818 & 1563 & 16,68 & 1182 & 12,61 & 2019 & 21,55 & 1442 & 15,39 & 1537 & 16,40 \\
\hline Tc_016254 & 767 & 10,69 & 710 & 9,89 & 3365 & 46,89 & 10312 & 143,69 & 2551 & 35,55 \\
\hline Tc_007045 & 733 & 26,38 & 602 & 21,66 & 922 & 33,18 & 1055 & 37,96 & 370 & 13,31 \\
\hline Tc_013894 & 2425 & 14,40 & 2095 & 12,44 & 609 & 3,62 & 2408 & 14,30 & 3092 & 18,36 \\
\hline
\end{tabular}


Table 13: Fold change for 32 iBeetle-identified + 2 enhancer trap screen genes, taken from Li (2013).

\begin{tabular}{|c|c|c|c|c|}
\hline \multirow{2}{*}{ OGS \# } & \multicolumn{4}{|c|}{ log2 Fold Change (sample reads/reference reads) } \\
\hline & $\mathrm{m}-$ the & $\mathrm{fm}-\mathrm{thx}$ & m-abd & fm-abd \\
\hline TC_000240 & $-4,12$ & $-3,66$ & $-4,12$ & $-2,95$ \\
\hline Tc_000379 & 1,26 & 1,18 & 1,18 & 1,58 \\
\hline Tc_000504 & 1,79 & 2,93 & 2,71 & 3,23 \\
\hline TC_000885 & $-0,12$ & $-0,47$ & $-1,54$ & $-3,97$ \\
\hline Tc_002616 & 0,35 & 0,29 & 0,41 & 1,02 \\
\hline Tc_002723 & $-0,77$ & $-1,08$ & $-0,29$ & 0,53 \\
\hline Tc_003409 & 1,21 & 1,37 & 1,07 & 1,48 \\
\hline TC_004129 & $-2,36$ & $-2,77$ & $-2,36$ & \\
\hline Tc_005167 & $-0,85$ & $-0,85$ & $-0,14$ & $-0,18$ \\
\hline Tc_006408 & $-0,91$ & $-0,90$ & $-0,75$ & $-0,83$ \\
\hline Tc_008608 & $-3,87$ & $-4,94$ & $-3,56$ & $-3,58$ \\
\hline Tc_010251 & 4,30 & 4,47 & 4,71 & 3,23 \\
\hline Tc_011075 & $-4,52$ & $-5,07$ & $-4,55$ & $-2,49$ \\
\hline TC_011288 & $-3,39$ & $-3,83$ & $-4,11$ & $-4,77$ \\
\hline Tc_011812 & 2,89 & 2,99 & 3,08 & 1,52 \\
\hline Tc_012539 & $-2,01$ & $-2,37$ & $-1,81$ & $-1,29$ \\
\hline TC_012610 & $-0,79$ & $-1,77$ & $-0,43$ & $-1,09$ \\
\hline TC_012828 & $-3,38$ & $-4,40$ & 0,14 & $-1,10$ \\
\hline Tc_012834 & $-1,05$ & $-1,22$ & $-0,47$ & $-0,94$ \\
\hline Tc_013513 & 4,40 & 4,53 & 5,30 & 3,28 \\
\hline Tc_013892 & $-0,92$ & $-1,18$ & $-0,74$ & $-0,04$ \\
\hline Tc_014494 & 0,29 & 0,40 & 0,55 & 0,32 \\
\hline Tc_014520 & $-0,88$ & $-1,04$ & $-0,83$ & $-0,98$ \\
\hline Tc_014870 & $-6,27$ & $-5,27$ & $-3,10$ & \\
\hline Tc_014887 & $-1,06$ & $-1,38$ & 0,21 & 2,42 \\
\hline Tc_015095 & 0,79 & 1,06 & 0,63 & 0,23 \\
\hline Tc_015165 & 1,19 & 1,10 & 1,21 & 0,82 \\
\hline Tc_015203 & 0,77 & 0,45 & 1,04 & $-1,78$ \\
\hline Tc_015379 & 2,22 & 1,88 & 4,08 & 4,60 \\
\hline Tc_015811 & 0,89 & 1,04 & 1,26 & 1,15 \\
\hline TC_015818 & 0,02 & $-0,38$ & 0,39 & $-0,09$ \\
\hline Tc_016254 & $-1,73$ & $-1,85$ & 0,40 & 2,02 \\
\hline Tc_007045 & 0,99 & 0,70 & 1,32 & 1,51 \\
\hline Tc_013894 & $-0,35$ & $-0,56$ & $-2,34$ & $-0,36$ \\
\hline
\end{tabular}




\section{APPENDIX}

\subsection{Signal-peptide analysis}

Table 14: Signal-peptide analysis of insect and mammalian peroxiredoxins. MTP: mitochondrial targeting peptide, SP: secretion pathway, other: nucleus/cytosol, P: peroxisome.

\begin{tabular}{|c|c|c|c|c|}
\hline \multirow{3}{*}{ Peroxiredoxins } & \multicolumn{3}{|c|}{$\mathrm{N}$-Terminal } & \multirow{3}{*}{\begin{tabular}{|c|} 
C-Terminal \\
PTS1 predictor \\
P/not P \\
\end{tabular}} \\
\hline & \multirow{2}{*}{$\begin{array}{c}\text { iPSORT } \\
\begin{array}{c}\mathrm{mTP} / \mathrm{SP} / \\
\text { other }\end{array} \\
\end{array}$} & \multicolumn{2}{|c|}{ TargetP } & \\
\hline & & $\begin{array}{c}\mathrm{mTP} / \mathrm{SP} / \\
\text { other }\end{array}$ & $\begin{array}{l}\text { prob- } \\
\text { ability }\end{array}$ & \\
\hline Dm PrxV & $\mathrm{mTP}$ & $\mathrm{mTP}$ & $85,70 \%$ & not $P$ \\
\hline Tc7642 & mTP & mTP & $51,80 \%$ & not $P$ \\
\hline Hs Prdx5_a & SP & mTP & $83,00 \%$ & $\mathrm{P}$ \\
\hline Hs Prdx5_b & SP & mTP & $84,30 \%$ & $\mathrm{P}$ \\
\hline Hs Prdx5_c & SP & mTP & $76,20 \%$ & $\mathrm{P}$ \\
\hline Mm Prdx5 & mTP & mTP & $89,60 \%$ & $\mathrm{P}$ \\
\hline Dm Dpx-5037 & mTP & mTP & $80,40 \%$ & not $P$ \\
\hline Tc12328 & mTP & mTP & $76,70 \%$ & not $P$ \\
\hline Hs Prdx3 & mTP & mTP & $83,40 \%$ & not $P$ \\
\hline Mm Prdx3 & $\mathrm{mTP}$ & mTP & $95 \%$ & not $P$ \\
\hline Dm Dpx-2540_1 & other & other & $83,50 \%$ & not $P$ \\
\hline Dm Dpx-2540_2 & other & other & $83,50 \%$ & not $P$ \\
\hline Dm Dpx-6005 & other & other & $87,40 \%$ & not $P$ \\
\hline Tc4948 & other & other & $81,70 \%$ & not $P$ \\
\hline Tc13791 & other & other & $91,60 \%$ & not $P$ \\
\hline Hs Prdx6 & other & other & $82,80 \%$ & not $P$ \\
\hline Mm Prdx6 & other & mTP/other & $46 \% / 31 \%$ & not $P$ \\
\hline Dm Dpx-4156 & SP & SP & $91,90 \%$ & not $P$ \\
\hline Tc1071 & SP & $\mathrm{SP}$ & $91,70 \%$ & not $P$ \\
\hline Hs Prdx4 & other & $\mathrm{SP}$ & $77,50 \%$ & not $P$ \\
\hline Mm Prdx4 & mTP & $\mathrm{SP}$ & $45,90 \%$ & not $P$ \\
\hline Dm Dpx-4783 & other & other & $51,90 \%$ & not $P$ \\
\hline Tc1700 & other & other & $59,30 \%$ & not $P$ \\
\hline Tc14929 & other & other & $67,20 \%$ & not $P$ \\
\hline Hs Prdx1 & other & other & $68,90 \%$ & not $P$ \\
\hline Hs Prdx2 & other & other & $59,00 \%$ & not $P$ \\
\hline Mm Prdx1 & other & other & $70,60 \%$ & not $P$ \\
\hline Mm Prdx2 & other & other & $73,60 \%$ & not $P$ \\
\hline
\end{tabular}




\subsection{Datasets of enzymatic activity assays}

Table 15: ABTS assay, pre-run.

\begin{tabular}{|r|r|r|}
\hline Time & $w t(a b d+t h x)+\mathrm{H}_{2} \mathrm{O}_{2}$ & wt $(\mathrm{abd}+\mathrm{thx})$ \\
\hline 0 & $-0,0758$ & $-\mathrm{H}_{2} \mathrm{O}_{2}$ \\
\hline 1 & $-0,0528$ & $-0,0953$ \\
\hline 5 & $-0,0206$ & $-0,0619$ \\
\hline 10 & 0,0137 & $-0,0638$ \\
\hline 20 & 0,1568 & $-0,0556$ \\
\hline 30 & 0,2538 & $-0,0583$ \\
\hline 40 & 0,4131 & $-0,0559$ \\
\hline 50 & 0,5801 & $-0,0597$ \\
\hline 60 & 0,6918 & $-0,0585$ \\
\hline
\end{tabular}

Table 16: Age-dependent ABTS assay.

\begin{tabular}{|c|c|c|c|c|c|c|c|c|c|}
\hline age & gland & $\mathrm{m} 1$ & $\mathrm{~m} 2$ & m3 & $\mathrm{m} 4$ & m5 & mean & $\begin{array}{l}\text { Standard } \\
\text { Deviation }\end{array}$ & $\begin{array}{l}\text { Standard Error } \\
\text { of the mean }\end{array}$ \\
\hline \multirow{4}{*}{ AO } & $\mathrm{fm}$ abd & 0,14 & 0,135 & 0,047 & 0,082 & 0,089 & 0,099 & 0,039 & 0,017 \\
\hline & $\mathrm{fm}$ thx & 0,098 & 0,07 & 0,118 & 0,124 & 0,107 & 0,103 & 0,021 & 0,009 \\
\hline & $\mathrm{m}$ abd & 0,102 & 0,131 & 0,122 & 0,112 & 0,087 & 0,111 & 0,017 & 0,008 \\
\hline & $m$ th $x$ & 0,07 & 0,091 & 0,09 & 0,072 & 0,148 & 0,094 & 0,032 & 0,014 \\
\hline \multirow{4}{*}{ A5 } & $\mathrm{fm}$ abd & 0,192 & 0,158 & 0,268 & 0,223 & 0,327 & 0,234 & 0,066 & 0,030 \\
\hline & fm thx & 0,153 & 0,214 & 0,158 & 0,246 & 0,191 & 0,192 & 0,039 & 0,017 \\
\hline & $\mathrm{m}$ abd & 0,21 & 0,192 & 0,185 & 0,2 & 0,138 & 0,185 & 0,028 & 0,012 \\
\hline & $m$ th $x$ & 0,189 & 0,221 & 0,139 & 0,165 & 0,252 & 0,193 & 0,045 & 0,020 \\
\hline \multirow{4}{*}{ A10 } & $\mathrm{fm}$ abd & 0,215 & 0,152 & 0,173 & 0,214 & 0,226 & 0,196 & 0,032 & 0,014 \\
\hline & $\mathrm{fm}$ thx & 0,139 & 0,119 & 0,167 & 0,22 & 0,15 & 0,159 & 0,038 & 0,017 \\
\hline & $\mathrm{m}$ abd & 0,205 & 0,188 & 0,168 & 0,154 & 0,162 & 0,175 & 0,021 & 0,009 \\
\hline & $m$ th $x$ & 0,162 & 0,121 & 0,125 & 0,154 & 0,164 & 0,145 & 0,021 & 0,009 \\
\hline \multirow{4}{*}{ A20 } & $\mathrm{fm}$ abd & 0,106 & 0,169 & 0,119 & 0,171 & 0,154 & 0,144 & 0,030 & 0,013 \\
\hline & fm thx & 0,1 & 0,098 & 0,064 & 0,089 & 0,073 & 0,085 & 0,016 & 0,007 \\
\hline & $\mathrm{m}$ abd & 0,197 & 0,139 & 0,137 & 0,134 & 0,16 & 0,153 & 0,026 & 0,012 \\
\hline & $m$ th $x$ & 0,066 & 0,117 & 0,091 & 0,083 & 0,107 & 0,093 & 0,020 & 0,009 \\
\hline \multirow{4}{*}{ A40 } & $\mathrm{fm}$ abd & 0,055 & 0,006 & 0,093 & 0,033 & 0,05 & 0,047 & 0,032 & 0,014 \\
\hline & fm thx & 0,045 & 0,035 & 0,055 & 0,056 & 0,016 & 0,041 & 0,017 & 0,007 \\
\hline & $\mathrm{m}$ abd & 0,086 & 0,032 & 0,01 & 0,046 & 0,073 & 0,049 & 0,031 & 0,014 \\
\hline & $m$ thx & 0,041 & 0,037 & 0,047 & 0,023 & 0,039 & 0,037 & 0,009 & 0,004 \\
\hline
\end{tabular}


APPENDIX

Table 17: ABTS assay with glands from RNAi knockdown beetles.

\begin{tabular}{|c|c|c|c|c|c|c|c|c|c|c|}
\hline \multirow{2}{*}{$\begin{array}{l}\text { A5 } \\
\text { Time }\end{array}$} & \multicolumn{2}{|c|}{ wt } & \multicolumn{2}{|c|}{ Lac2 RNAi } & \multicolumn{2}{|c|}{ Prdx3 RNAi } & \multicolumn{2}{|c|}{ Lac2A RNAi } & \multicolumn{2}{|c|}{ Lac2B RNAi } \\
\hline & abd & thx & abd & the & abd & thx & abd & the & abd & thx \\
\hline 0 & $-0,059$ & 0,019 & $-0,036$ & $-0,0084$ & $-0,013$ & $-0,0126$ & $-0,2475$ & $-0,02$ & $-0,1659$ & $-0,1833$ \\
\hline 1 & $-0,0565$ & 0,0255 & $-0,0302$ & $-0,0055$ & $-0,0091$ & $-0,0109$ & $-0,2776$ & $-0,0116$ & $-0,1664$ & $-0,1805$ \\
\hline 5 & $-0,0296$ & 0,048 & $-0,02713$ & & & 0,002 & & & & $-0,1782$ \\
\hline 10 & 0,0506 & 0,0844 & $-0,0255$ & & & 0,0242 & & & $-0,1807$ & $-0,1666$ \\
\hline 20 & 0,1529 & 265 & $-0,0277$ & 0,0246 & 0,1531 & 0,0793 & $-0,1415$ & 0,0186 & $-0,186$ & $-0,1336$ \\
\hline 30 & 0,2282 & 0,1716 & $-0,0254$ & 0,0587 & 0,2554 & 0,1345 & $-0,0749$ & 0,0476 & $-0,1885$ & $-0,1234$ \\
\hline 40 & 0,2921 & 0,2003 & $-0,0271$ & 0,0897 & 0,3136 & 0,1501 & $-0,0409$ & 0,0527 & $-0,1843$ & $-0,1156$ \\
\hline 50 & 0,3158 & 0,2152 & $-0,0249$ & 0,096 & 0,4315 & 0,1703 & $-0,0095$ & 0,0525 & $-0,1828$ & $-0,1051$ \\
\hline 60 & 0,3352 & 0,2343 & $-0,0133$ & 0,1075 & 0,5117 & 0,192 & 0,0364 & 0,058 & $-0,1816$ & $-0,1061$ \\
\hline$A_{420 \mathrm{~nm}}$ & 0,3492 & 0,2153 & 0,0227 & 0,1159 & 0,5247 & 0,2046 & 0,2839 & 0,078 & $-0,0157$ & 0,0772 \\
\hline
\end{tabular}

Table 18: Age-dependent DOPA assay.

\begin{tabular}{|c|c|c|c|c|c|c|c|c|c|}
\hline age & gland & $\mathrm{m} 1$ & $\mathrm{~m} 2$ & $\mathrm{~m} 3$ & $\mathrm{~m} 4$ & m5 & mean & St. Dev. & St. Error \\
\hline \multirow{4}{*}{$\mathrm{AO}$} & $\mathrm{fm}$ abd & 0,002 & 0,001 & 0,005 & 0,003 & 0,007 & 0,004 & 0,002 & 0,001 \\
\hline & fm thx & 0,006 & 0,004 & 0,008 & 0,005 & 0,007 & 0,006 & 0,002 & 0,001 \\
\hline & $m$ abd & 0,002 & 0,005 & 0,002 & 0,005 & 0,006 & 0,004 & 0,002 & 0,001 \\
\hline & $m$ thx & 0,01 & 0,006 & 0,005 & 0,011 & 0,005 & 0,007 & 0,003 & 0,001 \\
\hline \multirow{4}{*}{ A5 } & $\mathrm{fm}$ abd & 0,059 & 0,052 & 0,071 & 0,065 & 0,049 & 0,059 & 0,009 & 0,004 \\
\hline & $\mathrm{fm}$ thx & 0,018 & 0,013 & 0,011 & 0,003 & 0,019 & 0,013 & 0,006 & 0,003 \\
\hline & $\mathrm{m}$ abd & 0,037 & 0,032 & 0,038 & 0,033 & 0,032 & 0,034 & 0,003 & 0,001 \\
\hline & $m$ thx & 0,015 & 0,013 & 0,021 & 0,01 & 0,021 & 0,016 & 0,005 & 0,002 \\
\hline \multirow{4}{*}{ A10 } & $\mathrm{fm}$ abd & 0,045 & 0,049 & 0,042 & 0,052 & 0,041 & 0,046 & 0,005 & 0,002 \\
\hline & $\mathrm{fm}$ thx & 0,018 & 0,021 & 0,013 & 0,026 & 0,027 & 0,021 & 0,006 & 0,003 \\
\hline & $\mathrm{m}$ abd & 0,033 & 0,033 & 0,023 & 0,04 & 0,052 & 0,036 & 0,011 & 0,005 \\
\hline & $m$ thx & 0,007 & 0,008 & 0,018 & 0,019 & 0,033 & 0,017 & 0,011 & 0,005 \\
\hline \multirow{4}{*}{ A20 } & $\mathrm{fm}$ abd & 0,034 & 0,029 & 0,023 & 0,021 & 0,028 & 0,027 & 0,005 & 0,002 \\
\hline & fm thx & 0,013 & 0,011 & 0,021 & 0,017 & 0,023 & 0,017 & 0,005 & 0,002 \\
\hline & $\mathrm{m}$ abd & 0,012 & 0,017 & 0,034 & 0,019 & 0,017 & 0,020 & 0,008 & 0,004 \\
\hline & $m$ thx & 0,012 & 0,01 & 0,018 & 0,014 & 0,014 & 0,014 & 0,003 & 0,001 \\
\hline \multirow{4}{*}{ A40 } & $\mathrm{fm}$ abd & 0,04 & 0,025 & 0,059 & 0,046 & 0,037 & 0,041 & 0,012 & 0,006 \\
\hline & $\mathrm{fm}$ thx & 0,01 & 0,022 & 0,014 & 0,024 & 0,023 & 0,019 & 0,006 & 0,003 \\
\hline & $\mathrm{m}$ abd & 0,036 & 0,057 & 0,067 & 0,041 & 0,052 & 0,051 & 0,012 & 0,006 \\
\hline & $m$ thx & 0,002 & 0,003 & 0,026 & 0,019 & 0,014 & 0,013 & 0,010 & 0,005 \\
\hline
\end{tabular}


Table 19: DOPA assay with glands from RNAi knockdown beetles.

\begin{tabular}{|c|c|c|c|c|c|c|c|c|c|c|c|c|c|c|c|c|c|c|}
\hline \multirow[b]{2}{*}{$\begin{array}{c}\text { Glan } \\
\text { d } \\
\text { A10 }\end{array}$} & \multicolumn{3}{|c|}{ wt } & \multicolumn{3}{|c|}{ Lac2 RNAi } & \multicolumn{3}{|c|}{ Lac2A RNAi } & \multicolumn{3}{|c|}{ Lac2B RNAi } & \multicolumn{3}{|c|}{ DsRed RNAi } & \multicolumn{3}{|c|}{ Tyr1 RNAi } \\
\hline & $\begin{array}{c}\text { Abs. } \\
475 \\
\mathrm{~nm}\end{array}$ & StD & StE & $\begin{array}{c}\text { Abs. } \\
475 \\
\mathrm{~nm}\end{array}$ & StD & StE & $\begin{array}{c}\text { Abs. } \\
475 \\
\mathrm{~nm}\end{array}$ & StD & StE & \begin{tabular}{|c} 
Abs. \\
475 \\
$\mathrm{~nm}$
\end{tabular} & StD & StE & $\begin{array}{c}\text { Abs. } \\
475 \mathrm{~nm}\end{array}$ & StD & StE & \begin{tabular}{|c} 
Abs. \\
475 \\
$\mathrm{~nm}$
\end{tabular} & StD & StE \\
\hline $\begin{array}{r}f m \\
\text { abd }\end{array}$ & 0,046 & 0,005 & 0,002 & 0,008 & 0,004 & 0,002 & 0,025 & 0,005 & 0,002 & 0,037 & 0,011 & 0,005 & 0,063 & 0,024 & 0,011 & 0,031 & 0,013 & 0,006 \\
\hline $\begin{array}{r}m \\
a b d\end{array}$ & 0,036 & 0,011 & 0,005 & 0,011 & 0,007 & 0,003 & 02 & 0,006 & 0,003 & 0,053 & 0,025 & ,011 & , 055 & 0,017 & 0,008 & 0,017 & 0,002 & 0,001 \\
\hline $\begin{array}{r}f m \\
\text { thx }\end{array}$ & 0,021 & 0,006 & 0,003 & 0,002 & 0,002 & 0,001 & 0,009 & 002 & 0,001 & 0,029 & 0,007 & 0,003 &, 013 & 0,003 & 0,001 & 0,017 & 0,004 & 0,002 \\
\hline $\begin{array}{r}m \\
\text { thx }\end{array}$ & 0,017 & 0,011 & 0,00 & 0,004 & 0,003 & 0,001 & 0,015 & 0,003 & 0,001 & 0,031 & 0,009 & 0,004 & 0,015 & 0,008 & 0,00 & 0,011 & 0,017 & 0,0 \\
\hline
\end{tabular}

\subsection{Sequence alignment}

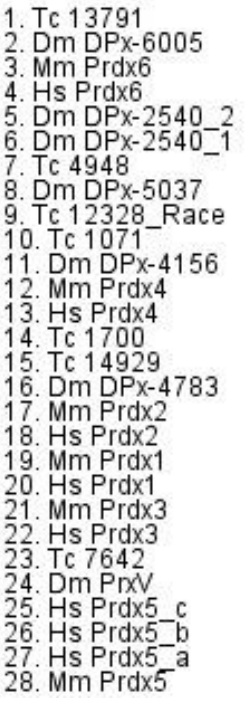

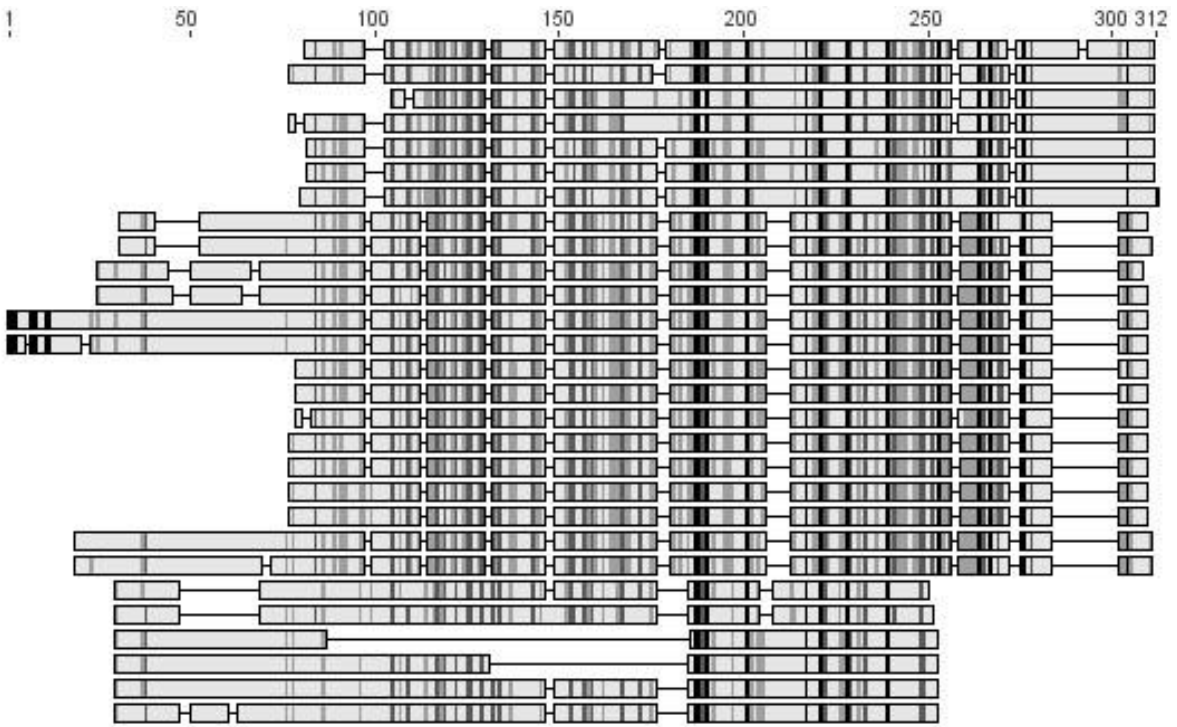

Figure 38: ClustalW alignment of peroxiredoxin full-length amino acid sequences. Dm: $D$. melanogaster, Hs: H. sapiens, Mm: M. musculus, Tc: T. castaneum. 


\section{APPENDIX}

\subsection{Raw data of GC-MS measurement}

Note that the values are not exact peak values, but estimated and rounded from the GC chromatograms. Abundances $<0.1 * 10^{6}$ were set to zero.

\subsection{1 iBeetle candidate genes}

Table 20: Abundances of volatiles in RNAi knockdown abdominal glands.

\begin{tabular}{|c|c|c|c|c|c|c|c|c|}
\hline \multirow{3}{*}{ OGS \# } & \multicolumn{8}{|c|}{ Abdominal gland abundance $\left({ }^{*} 10^{6}\right)$} \\
\hline & \multicolumn{4}{|c|}{ measurement 1 (male or mixed) } & \multicolumn{4}{|c|}{ measurement 2 (female or mixed) } \\
\hline & $M B Q$ & $E B Q$ & $1-C 15$ & $1-C 17$ & $M B Q$ & $E B Q$ & $1-C 15$ & $1-C 17$ \\
\hline Tc_000240 & 0,18 & 0,22 & 3 & 0,34 & 0 & 0 & 0,592 & 0 \\
\hline Tc_000379 & 1,2 & 1,04 & 4,69 & 0,86 & 0,96 & 1,08 & 4,84 & 0,8 \\
\hline Tc_000504 & 1,06 & 1,32 & 5,69 & 1,23 & 0,96 & 1,18 & 5,88 & 1,4 \\
\hline Tc_000885 & 0 & 0 & 0,172 & 0 & 0 & 0 & 1,14 & 0,19 \\
\hline Tc_002616 & 1,88 & 2,16 & 6 & 1,4 & 0,88 & 0,87 & 3,63 & 0,52 \\
\hline Tc_002723 & 0,68 & 0,92 & 6,08 & 1,2 & 0,84 & 0,86 & 5,36 & 0,72 \\
\hline Tc_003409 & 0,25 & 0,36 & 2,23 & 0,34 & $x x x$ & $x x x$ & $x x x$ & $x x x$ \\
\hline Tc_004129 & 2,07 & 2,65 & 7,3 & 1,3 & 0 & 0 & 0,66 & 0,124 \\
\hline Tc_005167 & 0 & 0 & 0,508 & 0,128 & $x x x$ & $x x x$ & $x x x$ & $x x x$ \\
\hline Tc_006408 & 0,72 & 0,88 & 4,99 & 1 & 0,64 & 0,68 & 5,94 & 1,24 \\
\hline Tc_008608 & 0,87 & 0,96 & 2,59 & 0,34 & 1,44 & 1,69 & 4,67 & 0,64 \\
\hline Tc_010251 & 0 & 0 & 6,5 & 1,42 & 0 & 0 & 2,82 & 0,38 \\
\hline Tc_011075 & 2,2 & 2,56 & 7,1 & 1,8 & 0,96 & 1,2 & 6 & 1,24 \\
\hline Tc_011288 & 0,34 & 0,4 & 3,25 & 0,62 & 0,24 & 0,28 & 4,15 & 0,48 \\
\hline Tc_011812 & 0 & 0 & 5,4 & 0,76 & 0 & 0 & 5 & 0,76 \\
\hline Tc_012539 & 0 & 0 & 1,65 & 0,25 & $x x x$ & $x x x$ & $x x x$ & $x x x$ \\
\hline Tc_012610 & 0,17 & 0,18 & 1,78 & 0,26 & $x x x$ & $x x x$ & $x x x$ & $\mathrm{xxx}$ \\
\hline Tc_012828 & 0,6 & 0,52 & 2,28 & 0,42 & 1,06 & 1 & 2,676 & 0,408 \\
\hline Tc_012834 & 0,96 & 0,9 & 4,94 & 0,66 & $x x x$ & $x x x$ & $x x x$ & $\mathrm{xxx}$ \\
\hline Tc_013513 & 0 & 0 & 0,36 & 0 & $x x x$ & $x x x$ & $x x x$ & $x x x$ \\
\hline Tc_013892 & 0,24 & 0,245 & 2,892 & 0,64 & 0,32 & 0,26 & 3 & 0,64 \\
\hline Tc_014494 & 2,35 & 2,5 & 6,5 & 1,32 & 0 & 0 & 1,35 & 0 \\
\hline Tc_014520 & 1,15 & 1,57 & 5,65 & 1,08 & 1,48 & 1,76 & 6,29 & 1,32 \\
\hline Tc_014870 & 0 & 0 & 0,264 & 0,172 & 0 & 0 & 1,42 & 0,3 \\
\hline Tc_014887 & 2,55 & 2,78 & 6,76 & 1,38 & 0,56 & 0,55 & 4,54 & 0,79 \\
\hline Tc_015095 & 1,24 & 0,84 & 5,24 & 0,8 & 0,46 & 0,38 & 4,08 & 0,52 \\
\hline Tc_015165 & 1,05 & 1,2 & 4,7 & 1 & 1 & 1,25 & 4,35 & 0,75 \\
\hline Tc_015203 & 0,58 & 0,68 & 5,64 & 0,9 & 1,49 & 1,55 & 8 & 1,29 \\
\hline Tc_015379 & 0,44 & 0,68 & 4,17 & 0,72 & 0 & 0 & 2,17 & 0,28 \\
\hline Tc_015811 & 2,46 & 2,51 & 9,8 & 2,2 & 0,44 & 0,44 & 2,54 & 0,32 \\
\hline Tc_015818 & 0 & 0 & 1,22 & 0,26 & 0 & 0 & 7,5 & 1,3 \\
\hline Tc_016254 & 0,66 & 0,76 & 4,77 & 0,8 & $x x x$ & $x x x$ & $x x x$ & $x x x$ \\
\hline
\end{tabular}

$1 \mathrm{~m}$ or $1 \mathrm{fm}$ in $50 \mu \mathrm{MeOH}$

$1 \mathrm{~m}+1 \mathrm{fm}$ in $100 \mu \mathrm{IeOH}$ 
Table 21: Abundances of volatiles in RNAi knockdown prothoracic glands.

\begin{tabular}{|c|c|c|c|c|c|c|c|c|}
\hline \multirow{3}{*}{ OGS \# } & \multicolumn{8}{|c|}{ Prothoracic gland abundance $\left({ }^{*} 10^{6}\right)$} \\
\hline & \multicolumn{4}{|c|}{ measurement 1 (male or mixed) } & \multicolumn{4}{|c|}{ measurement $\mathbf{2}$ (female or mixed) } \\
\hline & $M B Q$ & $E B Q$ & $1-C 15$ & $1-C 17$ & $M B Q$ & $E B Q$ & $1-C 15$ & $1-C 17$ \\
\hline Tc_000240 & 0 & 0 & 0,156 & 0 & 0 & 0 & 0,158 & 0 \\
\hline Tc_000379 & 0,365 & 0,36 & 3,7 & 1,04 & 0 & 0 & 1,4 & 0,37 \\
\hline Tc_000504 & 0,52 & 0,6 & 3,4 & 1,18 & 0,16 & 0,18 & 2,5 & 0,72 \\
\hline Tc_000885 & 0 & 0 & 0,278 & 0,112 & 0 & 0 & 0,153 & 0 \\
\hline Tc_002616 & 0 & 0 & 2,2 & 0,67 & 0,64 & 0,53 & 3,27 & 1,08 \\
\hline Tc_002723 & 0,46 & 0,45 & 3,44 & 0,96 & 0,76 & 0,65 & 4,72 & 1,46 \\
\hline Tc_003409 & 0,51 & 0,49 & 1,62 & 0,4 & $x x x$ & $x x x$ & $x x x$ & $x x x$ \\
\hline Tc_004129 & 0,15 & 0,17 & 0,86 & 0,52 & 0 & 0 & 0,138 & 0,1 \\
\hline Tc_005167 & 0 & 0 & 0,363 & 0,132 & $x x x$ & $x x x$ & $x x x$ & $x x x$ \\
\hline Tc_006408 & 0,2 & 0,2 & 4,26 & 1,48 & 0,26 & 0,32 & 4,37 & 1,57 \\
\hline Tc_008608 & 1,43 & 1,44 & 3,17 & 0,77 & 1,7 & 1,58 & 3,25 & 1 \\
\hline Tc_010251 & 0 & 0 & 1,552 & 0,406 & 0 & 0 & 4,42 & 1,36 \\
\hline Tc_011075 & 0,66 & 0,63 & 2,8 & 0,79 & 1,36 & 1,37 & 2,52 & 0,66 \\
\hline Tc_011288 & 0 & 0 & 0,4 & 0,172 & 0 & 0 & 0,895 & 0,24 \\
\hline Tc_011812 & 0 & 0 & 1,82 & 0,58 & 0 & 0 & 0,34 & 0,14 \\
\hline Tc_012539 & 0,53 & 0,56 & 1,69 & 0,48 & $x x x$ & $x x x$ & $x x x$ & $x x x$ \\
\hline Tc_012610 & 1,24 & 1,25 & 3,29 & 0,99 & $x x x$ & $x x x$ & $x x x$ & $\mathrm{xxx}$ \\
\hline Tc_012828 & 0 & 0 & 0,752 & 0,31 & 0,36 & 0,34 & 1,485 & 0,54 \\
\hline Tc_012834 & 0,65 & 0,72 & 2,38 & 0,78 & $x x x$ & $x x x$ & $x x x$ & $x x x$ \\
\hline Tc_013513 & 0 & 0 & 0,573 & 0,204 & $x x x$ & $x x x$ & $x x x$ & $x x x$ \\
\hline Tc_013892 & 0,1 & 0,1 & 1,34 & 0,42 & 0,1 & 0,09 & 0,93 & 0,33 \\
\hline Tc_014494 & 0,95 & 0,79 & 2,42 & 0,73 & 0,46 & 0,43 & 3,28 & 1 \\
\hline Tc_014520 & 0,52 & 0,64 & 3,34 & 0,86 & 0,32 & 0,42 & 3,66 & 1,1 \\
\hline Tc_014870 & 0 & 0 & 1,05 & 0,43 & 0,1 & 0,1 & 1,68 & 0,76 \\
\hline Tc_014887 & 0,54 & 0,5 & 3,28 & 1,04 & 0 & 0 & 2,91 & 0,92 \\
\hline Tc_015095 & 1,26 & 0,73 & 3,44 & 1,04 & 1,24 & 0,81 & 2,53 & 0,74 \\
\hline Tc_015165 & 0,4 & 0,48 & 2,18 & 1,32 & 0,44 & 0,62 & 2,12 & 0,7 \\
\hline Tc_015203 & 1,645 & 1,4 & 4,16 & 1,29 & 0,34 & 0,23 & 3,54 & 0,9 \\
\hline Tc_015379 & 0 & 0 & 1,095 & 0,35 & 0 & 0 & 0,524 & 0,156 \\
\hline Tc_015811 & 1,8 & 1,7 & 6,6 & 2,68 & 0,56 & 0,51 & 2,42 & 0,69 \\
\hline Tc_015818 & 0 & 0 & 1,25 & 0,45 & 0 & 0 & 1,49 & 0,45 \\
\hline Tc_016254 & 0,58 & 0,67 & 2,9 & 1 & $x x x$ & $x x x$ & $x x x$ & $x x x$ \\
\hline
\end{tabular}

$1 \mathrm{~m}$ or $1 \mathrm{fm}$ in $50 \mu \mathrm{MeOH}$

$1 \mathrm{~m}+1 \mathrm{fm}$ in $100 \mu \mathrm{MeOH}$ 
APPENDIX

Table 22: Abundances of volatiles in control glands (buffer injected beetles).

\begin{tabular}{|l|r|r|}
\hline & $\begin{array}{c}\text { inj. Buffer control } \\
\text { (msd0003) }\end{array}$ & $\begin{array}{c}\text { inj. Buffer control } \\
\text { (msd1000) }\end{array}$ \\
\hline \hline MBQ & 0,61 & 0,84 \\
\hline EBQ & 0,89 & 1,34 \\
\hline $1-C 15$ & 4,85 & 4,58 \\
\hline $1-C 17$ & 0,78 & 0,6 \\
\hline
\end{tabular}

$1 \mathrm{~m}$ or $1 \mathrm{fm}$ in $50 \mu \mathrm{MeOH}$

$1 \mathrm{~m}+1 \mathrm{fm}$ in $100 \mu \mathrm{MeOH}$

\subsubsection{Candidate genes from transcriptome data and enhancer trap screen}

Table 23: Abundances of volatiles in control glands (buffer-injected beetles).

\begin{tabular}{|l|c|r|}
\hline & $\begin{array}{c}\text { inj. buffer control } \\
\text { (mean msd } \\
1422+1424)\end{array}$ & $\begin{array}{c}\text { inj. buffer } \\
\text { control } \\
\text { (msd1000) }\end{array}$ \\
\hline \hline MBQ & 1,77 & 0,84 \\
\hline EBQ & 2,055 & 1,34 \\
\hline $1-C 15$ & 7,125 & 4,58 \\
\hline $1-C 17$ & 1,83 & 0,6 \\
\hline
\end{tabular}

$1 \mathrm{~m}$ or $1 \mathrm{fm}$ in $50 \mu \mathrm{MeOH}$

$3 \mathrm{~m}$ or $3 \mathrm{fm}$ in $100 \mu \mathrm{MeOH}$

Table 24: Abundances of volatiles in RNAi knockdown abdominal glands.

\begin{tabular}{|c|c|c|c|c|c|c|c|c|}
\hline \multirow{3}{*}{ OGS \# } & \multicolumn{8}{|c|}{ Abdominal gland abundance $\left(* 10^{6}\right)$} \\
\hline & \multicolumn{4}{|c|}{ male } & \multicolumn{4}{|c|}{ female } \\
\hline & $M B Q$ & $E B Q$ & $1-C 15$ & $1-C 17$ & $M B Q$ & $E B Q$ & $1-C 15$ & $1-C 17$ \\
\hline Tc_007045 & 1,7 & 1,92 & 6,04 & 1,08 & 0,17 & 0,19 & 2,23 & 0,29 \\
\hline Tc_013894 & 0 & 0 & 0 & 0 & 0,28 & 0,36 & 0,372 & 0,56 \\
\hline Tc_HEX1a & 1,4 & 1,68 & 6,32 & 1,44 & 1,52 & 2 & 5,2 & 0,92 \\
\hline Tc_Lac1 & 1,72 & 1,88 & 6,8 & 1,6 & 1,24 & 1,6 & 4,9 & 0,83 \\
\hline Tc_Lac2AB & 0 & 0 & 4,76 & 0,94 & 0 & 0 & 0,153 & 0 \\
\hline Tc_Lac2A & 0 & 0 & 1,21 & 0,17 & 0 & 0 & 4,12 & 0,5 \\
\hline Tc_Lac2B & 0,16 & 0,2 & 4,84 & 1,08 & 0,72 & 0,86 & 3,76 & 0,62 \\
\hline Tc_Tyr1 & 0,22 & 0,26 & 6,2 & 1,6 & 0,55 & 0,56 & 6,47 & 1,54 \\
\hline Tc_010355 & 1,04 & 0,95 & 4,94 & 1,04 & 0,66 & 0,68 & 4,05 & 0,58 \\
\hline Tc_010362 & 1,98 & 2,5 & 6,69 & 1,72 & 1,98 & 2,5 & 6,69 & 1,73 \\
\hline Tc_012328 & 0 & 0 & 4,6 & 0,9 & 0,2 & 0,2 & 6,65 & 1,7 \\
\hline
\end{tabular}

$1 \mathrm{~m}$ or $1 \mathrm{fm}$ in $50 \mu \mathrm{MeOH}$

$3 \mathrm{~m}$ or $3 \mathrm{fm}$ in $100 \mu \mathrm{l} \mathrm{MeOH}$ 
Table 25: Abundances of volatiles in RNAi knockdown prothoracic glands.

\begin{tabular}{|c|c|c|c|c|c|c|c|c|}
\hline \multirow{3}{*}{ OGS\# } & \multicolumn{8}{|c|}{ Prothoracic gland abundance $\left(* 10^{6}\right)$} \\
\hline & \multicolumn{4}{|c|}{ male } & \multicolumn{4}{|c|}{ female } \\
\hline & $M B Q$ & $E B Q$ & $1-C 15$ & $1-C 17$ & $M B Q$ & $E B Q$ & $1-C 15$ & $1-C 17$ \\
\hline Tc_007045 & 0,44 & 0,48 & 2,39 & 0,68 & 0,32 & 0,3 & 2,53 & 0,88 \\
\hline Tc_013894 & 0 & 0 & 1,3 & 0,42 & 0 & 0 & 0,274 & 0,115 \\
\hline Tc_HEX1a & 0,48 & 0,66 & 3,48 & 1,24 & 0,64 & 0,86 & 3,68 & 1,28 \\
\hline Tc_Lac1 & 0,15 & 0,2 & 2,15 & 0,61 & 0,16 & 0,22 & 3,2 & 1,05 \\
\hline TC_Lac $2 A B$ & 0 & 0 & 0,85 & 0,3 & 0 & 0 & 2,03 & 0,7 \\
\hline Tc_Lac2A & 0 & 0 & 0,62 & 0,19 & 0 & 0 & 0,41 & 0,11 \\
\hline Tc_Lac2B & 0 & 0 & 2,28 & 0,78 & 0,44 & 0,63 & 3,6 & 1,21 \\
\hline Tc_Tyr1 & 0,26 & 0,26 & 4,52 & 1,64 & 0,2 & 0,2 & 5,39 & 2,44 \\
\hline Tc_010355 & 0,22 & 0,19 & 2,39 & 0,8 & 0,44 & 0,36 & 3,01 & 1 \\
\hline Tc_010362 & 0,64 & 1 & 3,92 & 1,42 & 0,68 & 0,9 & 4,16 & 1,4 \\
\hline Tc_012328 & 0 & 0 & 3,8 & 1,45 & 0 & 0 & 5,69 & 2,27 \\
\hline
\end{tabular}

$1 \mathrm{~m}$ or $1 \mathrm{fm}$ in $50 \mu \mathrm{MeOH}$

$3 \mathrm{~m}$ or $3 \mathrm{fm}$ in $100 \mu \mathrm{MeOH}$

\subsection{RACE of Tc_012328}

\section{Nucleotide sequence:}

ggtgctcaaagtccctagacttggcactgactttccaaattttgataattaatttcagcgactcatcgcac ttttaaacaaatttgctggcagtatcaacgttttgttataatggctggactatttagcacaattgtacgac ggggcccccaattgttaaaaactgcgattccggctggtaaaaacaatgcatttagaaatttttccgttgcc cccactttgtgcgctcctcgagtgcaacaccccgctcctgactttaaaggaacagcagttataaatgatgg ctttaaagagatacaattgagcgactataagggaaaatatgttgttctagttttctatcctcttgatttca cttttgtgtgccccactgaattgatagcgttagatgaacgttacgacgatttcaaaacttgaacgctgaa gtaattggttgttctattgattcacatttctcgcacttagggtggatgaacacaaaagatctgaaggggg tttgggcaaactaagatatcccttgctttctgacattaataaaactatagccagagactatgatgtcctgc tcgagaaagagggaattgccttgagaggtcttttcattattgaccctaatggcatccttaggcaaattacg gtaaatgatttgccaataggtcgttccgtagatgaggctcttcggttaatcgaagccattcaattctttga aaaaatggagaagtgtgtccagctaattggaagaaaggtagtaagacaatcaaacctgaccctcaaggat ctaagaatatttccaagccgctaataagtaatgagtaaacgttttgtgtttgtaaattgttacgttgtaa agatttgaataatataacttaat

5'UTR, 3’UTR, start, stop

\section{Amino acid sequence:}

MAGLFSTIVRRGPQLLKTAIPAGKNNAFRNFSVAPTLCAPRVQHPAPDFKGTAVINDGFKE IQLSDYKGKY VVLVFYPLDFTFVCPTELIALDERYDDFKNLNAEVIGCS IDSHFSHLGWMNTKRSEGGLGKLRYPLLSDIN KTIARDYDVLLEKEGIALRGLFIIDPNGILRQITVNDLPIGRSVDEALRLIEAIQFFEKNGEVCPANWKKG SKTIKPDPQGSKEYFQAANK 

